\title{
CHARACTERISING THE RESPONSE OF PLANT-MYCORRHIZAL NETWORKS TO A CHANGING ALPINE ENVIRONMENT
}

\author{
BY \\ LESLEY MICHELLE WILD
}

\begin{abstract}
A thesis
submitted to the Victoria University of Wellington

in fulfilment of the requirements for the degree of Master of Science

in the field of Ecology and Biodiversity
\end{abstract}

Victoria University of Wellington

December 2020 



\begin{abstract}
Anthropogenic climate change effects are particularly acute in alpine ecosystems. New Zealand's alpine regions are experiencing climatic changes at higher than global mean rates, particularly warming and drying. These communities are also facing increasing rates of invasion by exotic plant species. Notably, multiple drivers of change, such as warming and invasion, have been evidenced to interact and facilitate greater ecosystem change. This is of particular concern as New Zealand's alpine plant communities are unique globally and represent national hotspots of biodiversity. Therefore there is a pressing need to understand how they may be affected by the independent and interactive drivers of global environmental change. Alpine plant species form ubiquitous and obligate symbiotic associations with mutualistic mycorrhizal fungi. Plant-mycorrhiza networks are foundational interactions that underpin the diversity and function of terrestrial communities. Plant-mycorrhiza networks are also particularly sensitive to temperature shifts and plant invasions. In this thesis, I investigate the independent and interactive effects of warming and the presence of an invasive species (Common Heather, Calluna vulgaris) on the fungal community composition and the network of mycorrhiza interactions of alpine plants in Tongariro National Park, New Zealand. I sampled the roots of plant species within the Warming and species Removal in Mountains (WaRM) experiment, a factorial combination of warming and Calluna vulgaris removals $(\mathrm{n}=8$ per treatment) established in TNP in 2015. The plant community at the site consists of plant species that form either arbuscular mycorrhizas or ericoid mycorrhizas. I selected the three most abundant plant species of each mycorrhizal type at the site scale for sampling in each of the 32 plots. DNA was extracted from plant roots,
\end{abstract}


and the internal transcribed spacer of the fungal rRNA gene was amplified by PCR and sequenced on the Illumina Mi-seq platform. Sequence data was demultiplexed and fungal OTUs were identified using the PIPITS pipeline, referencing the UNITE fungal database. In my second chapter, I consider plant species and treatment effects on the diversity and community composition of mycorrhizal fungi. I found WaRM treatments were significant determinants of mycorrhizal compositions in host plant species. Warming simultaneously increased the mycorrhizal fungal diversity and richness of invasive Calluna vulgaris and reduced that of the native host plant species. In chapter 3, using network analyses from the bipartite package of $\mathrm{R}$, I constructed 32 plant mycorrhizal networks of the plot sampled and calculated metrics pertaining to properties of network structure and robustness at the whole network, trophic-level and species/mycorrhizal fungal OTU scales. I then examined the responses of these metrics to the WaRM treatments. I found that warming significantly reduced the robustness of native plant-mycorrhizal networks and increased the strength of the interaction network associated with invasive $C$. vulgaris. The removal of $C$. vulgaris had a secondary effect on how mycorrhizal fungal compositions and interaction networks responded to warming. As a generalist $C$. vulgaris was critical for the ongoing diversity of ericoid mycorrhizal fungi, particularly under warming. However, C. vulgaris simultaneously suppressed the mycorrhizal interactionnetworks of native plant species, which further fragmented under warming. I conclude that warming and the presence of invasive C. vulgaris synergistically reduced and decentralised the native plant-mycorrhizal interactions within the network. In summary, my thesis demonstrates the below-ground interactions of alpine plant communities are destabilising under multiple interacting drivers of global environmental change. 


\section{Acknowledgments}

My sincere and deepest gratitude to my supervisor Dr Julie Deslippe and her phenomenal knowledge, support and advice. I would also like to extend my most enthusiastic thank you to Natascha Lewe for her guiding hand with the molecular and bioinformatics methods used in this thesis. I would also like to acknowledge the hard work of Justyna Giejsztowt for helping to maintain the WaRM experiment in the three years preceding my sampling of it as well as her wealth of knowledge of in Tongariro National Park. To Danyl McLauchlan, thank you for your patience and wonderful teaching ability which helped me to overcome my fear of bioinformatics. To my family and the Richards family, your never-ending support has ensured I've made it to this finishing line. 


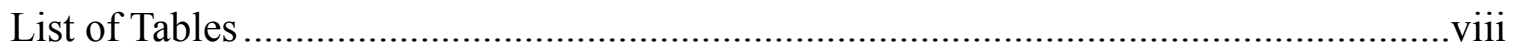

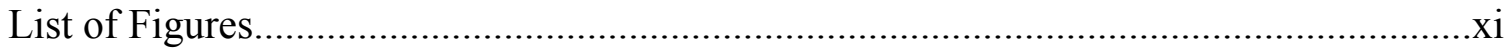

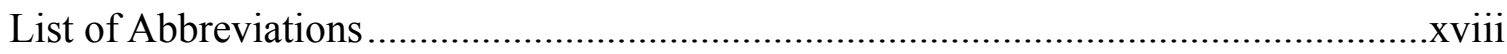

List of Species used in this study.........................................................................

Chapter I. Literature Review, Aims and Hypotheses...........................................................1

Literature Review ............................................................................

Statement of Study's Contribution, Aims and Hypotheses ...........................27

Chapter II. The effect of warming and invasive species removal on a ericoid and arbuscular mycorrhizal fungi community structure in alpine tussock grassland.....29

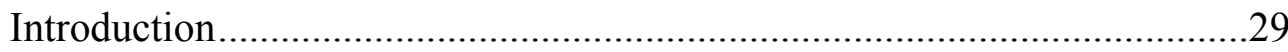

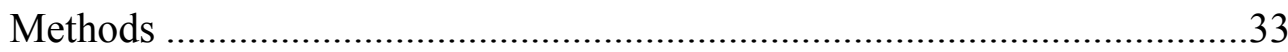

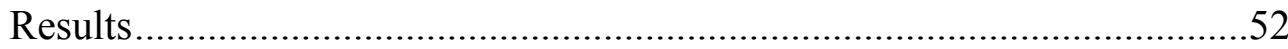

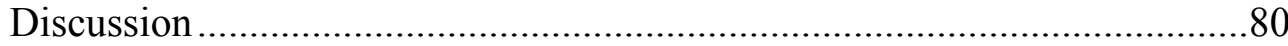

Chapter III. The effects of warming and dominant species removal on plantmycorrhizal fungal interaction networks in an alpine tussock grassland....................85

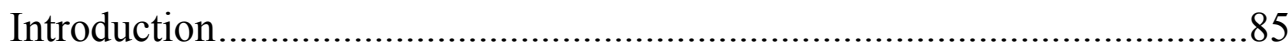

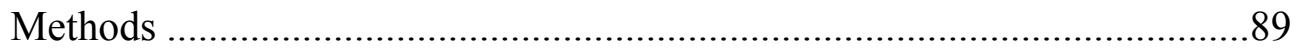

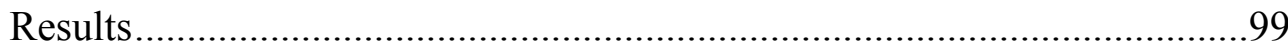

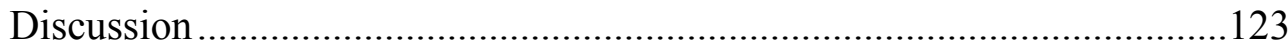

Chapter IV. Thesis Review, Discussion and Synthesis......................................................132

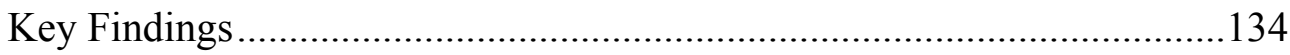

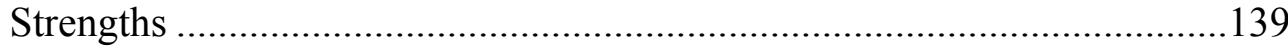

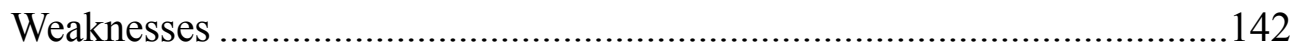

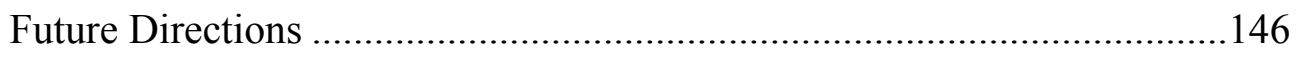

Synthesis Statements.............................................................................149

Appendix 1. Supplementary Tables............................................................................150

Appendix 2. Supplementary Figures …………………….........................................157

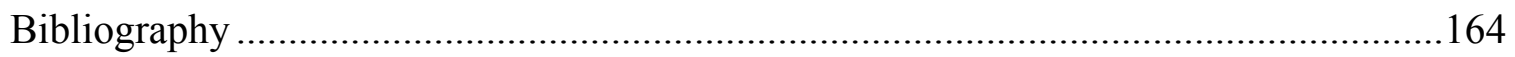




\section{List of Tables}

Table 1 Mean percentage cover of host plant species in the WaRM treatments. Number of individual host plants sampled per species sampled from the WaRM treatments; $\mathrm{n}=8$ plots per treatment. CALVUL, Calluna vulgaris CHIRUB, Chionochloa rubra. DRASUB, Dracophyllum subulatum. EPAALP, Epacris alpina. OZOVAU, Ozothamnus vauvilliersii. VERVEN, Veronica venustula.

Table 2 Number of individual host plants successfully sampled per species from the WaRM treatments; $\mathrm{n}=8$ plots per treatment. CALVUL, Calluna vulgaris CHIRUB, Chionochloa rubra. DRASUB, Dracophyllum subulatum. EPAALP, Epacris alpina. OZOVAU, Ozothamnus vauvilliersii. VERVEN, Veronica venustula.

Table 3 Two-Way ANOVA results of ErM fungal OTU diversity (Shannon Diversity Index, Species Richness and Pielou's Evenness) in ErM host plant species. CALVUL, Calluna vulgaris. DRASUB, Dracophyllum subulatum. EPAALP, Epacris alpina. Treatment effects from the TwoWay ANOVA are denoted as an increase with $(+)$ or $(-)$ with significance noted as $* * *$ if $\mathrm{p}<0.0005, * *$ if $\mathrm{p}<0.005, *$ if $\mathrm{p}<0.05$, $\mathrm{t}$ if $\mathrm{p}<0.10$.

Table 4 Two-Way ANOVA results of AM fungal OTU diversity (Shannon Diversity Index, Species Richness and Pielou's Evenness) in AM host plant species. CHIRUB, Chionochloa rubra. OZOVAU, Ozothamnus vauvilliersii. VERVEN, Veronica venustula. Treatment effects from the Two-Way ANOVA are denoted as an increase with $(+)$ or $(-)$ with significance noted as $* * *$ if $\mathrm{p}<0.0005, * *$ if $\mathrm{p}<0.005, *$ if $\mathrm{p}<0.05$, $\mathrm{t}$ if $\mathrm{p}<0.10$.

Table 5 Pairwise differences of Multiple Response Permutation Procedure (MRPP) of all treatments $(n=4)$ by mycorrhizal OTUs $(\mathrm{p}=100)$. Significant difference between treatments detected with MRPP are denoted with $* * *$ if $\mathrm{p}<0.0005, * *$ if $\mathrm{p}<0.005, *$ if $\mathrm{p}<0.05$, $\mathrm{t}$ if $\mathrm{p}<0.10$.

Table 6 Pairwise differences of Multiple Response Permutation Procedure (MRPP) of all host-plant species $(n=6)$ by mycorrhizal OTUs ( $p$ $=100)$. CALVUL, Calluna vulgaris CHIRUB, Chionochloa rubra. DRASUB, Dracophyllum subulatum. EPAALP, Epacris alpina. OZOVAU, Ozothamnus vauvilliersii. VERVEN, Veronica venustula. Significant difference between treatments detected with MRPP are denoted with $* * *$ if $\mathrm{p}<0.0005, * *$ if $\mathrm{p}<0.005, *$ if $\mathrm{p}<0.05$, $\mathrm{t}$ if $\mathrm{p}<0.10$. 
Table 7 Pairwise differences of Multiple Response Permutation Procedure (MRPP) of all host-plant species $(\mathrm{n}=6)$ by ErM OTUs $(\mathrm{p}=47)$. CALVUL, Calluna vulgaris CHIRUB, Chionochloa rubra. DRASUB, Dracophyllum subulatum. EPAALP, Epacris alpina. OZOVAU, Ozothamnus vauvilliersii. VERVEN, Veronica venustula. Significant difference between treatments detected with MRPP are denoted with $* * *$ if $\mathrm{p}<0.0005, * *$ if $\mathrm{p}<0.005, *$ if $\mathrm{p}<0.05$.

Table 8 Pairwise differences of Multiple Response Permutation Procedure (MRPP) of all host-plant species $(\mathrm{n}=6)$ by AMF OTUs $(\mathrm{p}=53)$. CALVUL, Calluna vulgaris CHIRUB, Chionochloa rubra. DRASUB, Dracophyllum subulatum. EPAALP, Epacris alpina. OZOVAU, Ozothamnus vauvilliersii. VERVEN, Veronica venustula. Significant difference between treatments detected with MRPP are denoted with $* * *$ if $\mathrm{p}<0.0005, * *$ if $\mathrm{p}<0.005, *$ if $\mathrm{p}<0.05$.

Table 9 Pairwise differences of Multiple Response Permutation Procedure (MRPP) of all ErM host-plant species by treatment $(n=4)$ by ErM OTUs ( $\mathrm{p}=47)$ CALVUL, Calluna vulgaris. DRASUB, Dracophyllum subulatum. EPAALP, Epacris alpina. Significant difference between treatments detected with MRPP are denoted with $* * *$ if $\mathrm{p}<0.0005, * *$ if $\mathrm{p}<0.005, *$ if $\mathrm{p}<0.05$.

Table 10 Pairwise differences of Multiple Response Permutation Procedure (MRPP) between ErM host-plant species $(\mathrm{n}=3)$ by ErM OTUs $(\mathrm{p}=$ 53). CALVUL, Calluna vulgaris. DRASUB, Dracophyllum subulatum. EPAALP, Epacris alpina. Significant difference between treatments detected with MRPP are denoted with $* * *$ if $\mathrm{p}<0.0005$, $* *$ if $\mathrm{p}<0.005$, $*$ if $\mathrm{p}<0.05$.

Table 11 Pairwise differences of Multiple Response Permutation Procedure (MRPP) of AM host-plant species by treatment $(\mathrm{n}=4)$ by AMF OTUs $(\mathrm{p}=53)$ CHIRUB, Chionochloa rubra. OZOVAU, Ozothamnus vauvilliersii. VERVEN, Veronica venustula. Significant difference between treatments detected with MRPP are denoted with *** if $\mathrm{p}$ $<0.0005, * *$ if $\mathrm{p}<0.005, *$ if $\mathrm{p}<0.05$.

Table 12 Pairwise differences of Multiple Response Permutation Procedure (MRPP) between all AM host-plant species $(\mathrm{n}=3)$ by AMF OTUs $(\mathrm{p}=$ 53). CHIRUB, Chionochloa rubra. OZOVAU, Ozothamnus vauvilliersii. VERVEN, Veronica venustula. Significant difference between treatments detected with MRPP are denoted with *** if $\mathrm{p}$ $<0.0005, * *$ if $\mathrm{p}<0.005, *$ if $\mathrm{p}<0.05$. 
Table 13 Description, calculation and interpretation of the network level metrics calculated for each plot $(n=32)$.

Table 14 Description, calculation and interpretation of the trophic level analysis indices calculated for each plot $(n=32)$.

Table 15 Description, calculation and interpretation of the species level metrics calculated for each plot $(n=141)$.

Table 16 Two-Way ANOVA results of species-level "structure" metrics by hostplant species. CALVUL, Calluna vulgaris. DRASUB, Dracophyllum subulatum. EPAALP, Epacris alpina. CHIRUB, Chionochloa rubra. OZOVAU, Ozothamnus vauvilliersii. VERVEN, Veronica venustula. Significant treatment effects from the Two-Way ANOVA are denoted $* * *$ if $\mathrm{p}<0.0005, * *$ if $\mathrm{p}<0.005, *$ if $\mathrm{p}<0.05, \mathrm{t}$ if $\mathrm{p}<0.10$ with "+" for increase and "-" for decrease effect. Full results of the Two-Way ANOVA are listed in Appendix 1. Supplementary Table 7.

Table 17 Two-Way ANOVA results of species-level "robustness" metrics by hostplant species. CALVUL, Calluna vulgaris. DRASUB, Dracophyllum subulatum. EPAALP, Epacris alpina. CHIRUB, Chionochloa rubra. OZOVAU, Ozothamnus vauvilliersii. VERVEN, Veronica venustula. Significant treatment effects from the Two-Way ANOVA are denoted $* * *$ if $\mathrm{p}<0.0005, * *$ if $\mathrm{p}<0.005, *$ if $\mathrm{p}<0.05, \mathrm{t}$ if $\mathrm{p}<0.10$ with "+" for increase and "-" for decrease effect. Full results of the Two-Way ANOVA are listed in Appendix 1. Supplementary Table 8. 


\section{List of Figures}

Figure 1 (Perotto et al., 2018) Morphological comparison between intracellular root tissue colonisation of ericoid mycorrhizas and arbuscular mycorrhizas. Notably, arbuscular mycorrhizas are distinguished from their formation spherical storage organs called vesicles and sites of nutrient exchange called arbuscules.

Figure 2 Wahl and Spiegelberger, (2016) demonstrate how plant-MF interactions can shift in their symbiotic nature along an elevational gradient in an alpine system due to the abiotic limitations (left). They illustrate how nature of the plant-MF interactions may alter under increased abiotic limitations due to factors of global change (right).

Figure 3 Bücking et al., (2016) illustrate how the formations of common mycorrhizal networks can elicit interactions that strongly and asymmetrically influence host plant and MF fitness within a naturally assembled community.

Figure 4. Dorman et al., (2009) demonstrate bipartite interaction networks constructed with the plotweb function from the bipartite package in R. "Lower Trophic level" species (interaction partners), are connected to the species the "Higher Trophic level" (source of interaction). The width of the bar for each species (i.e., node) is proportional to the relative abundance of interactions it has, and acts as a proxy for the relative influence a species exerts on the network (i.e., its centrality). The width of each link is proportional to the relative strength of the interaction, typically quantified as the number of interactions observed between the partners.

Figure 5 Blüthgen and Klein, (2011) demonstrate how the diversity of interactions exhibited by network participants occupy niche space, characterising their function. "Functional redundancy" occurs where participants A and B mostly overlap in their function role, meaning that there is significant similarity in the interactions they exhibit. Complementary functions arise when species A and B are dissimilar in their topology of interactions, representative of "functional diversity". The higher degree both measures are in a network, the higher "functional complementarity" and robustness of the network.

Figure 6 Diagram of the Warming and Dominant Species Removal (WaRM) experimental design. Green "plants" represent the removal of dominant plants. Warming plots demonstrate the application of Open-Top Chambers that passively warm the plots by $\sim 1.73^{\circ} \mathrm{C}$ higher than the controls. The factorial design resulting in 4 treatments. These treatment are ambient-control, ambient-removal, warm-control, warm-removal. 
Figure 7 Satellite Image (Google Earth, earth.google.com/web) of Tongariro National Park illustrating the location of the WaRM experimental site. The site is located at $\sim 1000 \mathrm{~m}$ elevation on the Rangipo desert on the eastern leeward side of Mount Ruapehu, where C. vulgaris is still expanding in its spatial range.

Figure 8 Diagram of the Warming and Dominant Species Removal (WaRM) experimental design. Green "plants" represent C. vulgaris. Warming plots demonstrate the application of Open-Top Chambers that passively warm the plots by $\sim 1.73^{\circ} \mathrm{C}$ higher than the controls. WaRM consists of 8 replicates plots of each treatment: ambient-control; ambient-removal; warming-control; warming-removal $(n=32)$.

Figure 9 The cumulative number of fungal OTUs detected as a function of the number of plant root samples (i.e., a Collector's accumulation curve), using the collectors.curve from the plot.rtk package in $\mathrm{R}$. The flattening of the curve and narrowing of standard error suggests that I have sufficiently sampled the fungal community associated with the roots of 6 alpine plant species.

Figure 10 Fungal OTU accumulation by WaRM treatments as a function of the number of sequence reads (i.e., rarefaction curve), from the rare.curve function from the vegan package in $\mathrm{R}$.

Figure 11 The proportional abundance of fungal functional guilds in the WaRM treatments as determined by (A) read count and (B) OTU richness. OTU richness was calculated using the vegan package in $\mathrm{R}$.

Figure 12 Fungal OTU accumulation by host plant species as a function of the number of sequence reads (i.e., rarefaction curve) from the rare.curve function from the vegan package in $\mathrm{R}$. Note that Calluna vulgaris (CALVUL) is removed from half of the plots. CALVUL, Calluna vulgaris CHIRUB, Chionochloa rubra. DRASUB, Dracophyllum subulatum. EPAALP, Epacris alpina. OZOVAU, Ozothamnus vauvilliersii. VERVEN, Veronica venustula.

Figure 13 The proportional abundance of fungal functional guilds in the host plant species as determined by (A) read count and (B) OTU richness. OTU richness was calculated using the vegan package in R. CALVUL, Calluna vulgaris CHIRUB, Chionochloa rubra. DRASUB, Dracophyllum subulatum. EPAALP, Epacris alpina. OZOVAU, Ozothamnus vauvilliersii. VERVEN, Veronica venustula.

Figure 14 Mycorrhizal fungal (MF) OTU accumulation by WaRM treatments as a function of the number of sequence reads (i.e., rarefaction curve), from the rare.curve function from the vegan package in $\mathrm{R}$. 
Figure 15 Standardised sequence reads of AMF and ErM fungi by species. Read counts are standardised to the mean of the total number of reads by species.

Figure 16 Mycorrhizal fungal (MF) OTU accumulation by host plant species as a function of the number of sequence reads (i.e., rarefaction curve) from the rare.curve function from the vegan package in $\mathrm{R}$. Note that CALVUL is removed from half of the plots. CALVUL, Calluna vulgaris CHIRUB, Chionochloa rubra. DRASUB, Dracophyllum subulatum. EPAALP, Epacris alpina. OZOVAU, Ozothamnus vauvilliersii. VERVEN, Veronica venustula.

Figure 17 Standardised sequence reads of AMF and ErM fungi by species. Read counts are standardised to the mean of the total number of reads by species. CALVUL, Calluna vulgaris. CHIRUB, Chionochloa rubra. DRASUB, Dracophyllum subulatum. EPAALP, Epacris alpina. OZOVAU, Ozothamnus vauvilliersii. VERVEN, Veronica venustula.

Figure 18 Diversity indices (Shannon's Diversity, Richness, and Pielou's Evenness) of ErM OTUs associated with three ericoid mycorrhizal host plant species in response to factorial warming and removal treatment ( $\mathrm{n}=8$ per treatment, 32 total). Median values for each metric are denoted by a horizontal line, boxes represent the interquartile ranges and whiskers denote the upper and lower 95\% confidence intervals for each metric in response to the four treatments. Significant treatment effects from the Two-Way ANOVA are noted with $* * *$ if $\mathrm{p}<0.0005$, ** if $\mathrm{p}<0.005, *$ if $\mathrm{p}<0.05$. CALVUL, Calluna vulgaris. DRASUB, Dracophyllum subulatum. EPAALP, Epacris alpina. WxR, Warming and Removal factors as a significant treatment effect.

Figure 19 Diversity indices (Shannon's Diversity, Richness, and Pielou's Evenness) of AM OTUs associated with three arbuscular mycorrhizal host plant species in response to factorial warming and removal treatment ( $\mathrm{n}=8$ per treatment, 32 total). Median values for each metric are denoted by a horizontal line, boxes represent the interquartile ranges and whiskers denote the upper and lower 95\% confidence intervals for each metric in response to the four treatments. Significant treatment effects from the Two-Way ANOVA are noted with $* * *$ if $\mathrm{p}$ $<0.0005, * *$ if $\mathrm{p}<0.005, *$ if $\mathrm{p}<0.05$. CHIRUB, Chionochloa rubra. OZOVAU, Ozothamnus vauvilliersii. VERVEN, Veronica venustula.

Figure 20 NMS graph with all plant root samples $(n=141)$ arranged in mycorrhizal OTU $(\mathrm{p}=100)$ ordination space by treatment $(\mathbf{A})$ and by species (B). CALVUL, Calluna vulgaris CHIRUB, Chionochloa rubra. DRASUB, Dracophyllum subulatum. EPAALP, Epacris alpina. OZOVAU, Ozothamnus vauvilliersii. VERVEN, Veronica venustula. 
Figure 21 NMS graphs with all host-plant species $(n=141)$ arranged in mycorrhizal OTU ordination space by ErM OTUs $(\mathrm{p}=47)(\mathrm{A})$ and AMF OTUs $(\mathrm{p}=53)$ (B). CALVUL, Calluna vulgaris CHIRUB, Chionochloa rubra. DRASUB, Dracophyllum subulatum. EPAALP, Epacris alpina. OZOVAU, Ozothamnus vauvilliersii. VERVEN, Veronica venustula.

Figure 22 NMS graphs with all host plant samples that form ericoid mycorrhizal associations $(n=67)$ arranged in ericoid mycorrhizal (ErM) fungi OTU ( $\mathrm{p}=47$ ) ordination space by treatment (A) and by species (B). CALVUL, Calluna vulgaris. DRASUB, Dracophyllum subulatum. EPAALP, Epacris alpina.

Figure 23 NMS graphs by host plant species that form ericoid mycorrhizal associations, CALVUL, Calluna vulgaris $(\mathrm{n}=14)(\mathbf{A})$, DRASUB, Dracophyllum subulatum $(\mathrm{n}=29)(\mathbf{B})$, EPAALP, Epacris alpina $(\mathrm{n}=24)(\mathbf{C})$, arranged in ericoid mycorrhizal ( ErM ) fungi OTU ( $\mathrm{p}=$ 47) ordination space by treatment. Axes are labeled as the proportion of variation explained, all MRPP pairwise differences are listed in Appendix 1, Tables 2-3.

Figure 24 NMS graphs with all host plant samples that form arbuscular mycorrhizal associations $(\mathrm{n}=74)$ arranged in arbuscular mycorrhizal (AM) fungi OTU $(\mathrm{p}=53)$ ordination space by treatment $(\mathbf{A})$ and by species (B) CHIRUB, Chionochloa rubra. OZOVAU, Ozothamnus vauvilliersii. VERVEN, Veronica venustula.

Figure 25 NMS graphs by host plant species that form arbuscular mycorrhizal associations, CHIRUB, Chionochloa rubra $(\mathrm{n}=24)(\mathbf{A})$, OZOVAU, Ozothamnus vauvilliersii $(\mathrm{n}=28)(\mathrm{B})$, VERVEN, Veronica venustula $(\mathrm{n}=22)(\mathbf{C})$ arranged in arbuscular mycorrhizal (AM) fungi OTU $(\mathrm{p}=53)$ ordination space by treatment. Axes are labeled as the proportion of variation explained, all MRPP pairwise differences are listed in Appendix 1, Tables 4-6. 
Figure 26 Mean bipartite plant-mycorrhizal networks by treatment $(\mathbf{n}=\mathbf{8})$ constructed with the plotweb function from the bipartite package in R. Host plants are on the "lower level" (lower trophic rank), connected to the ErM and AM fungal OTUs in the "higher level" (higher trophic rank). The width of the bar (i.e., graph node) for each network participant is proportional to the relative strength and diversity of its interactions, and acts as a proxy for the relative centrality (influence) of the participant on the network. The width of each link (interaction) is proportional to the relative strength of the interaction, quantified as the number of fungal OTU sequence reads that link the fungal OTU and plant species. Fungal OTUs are ordered from left to right firstly by ErM and AM fungi, and then in order of their overall rank abundance across all samples. CALVUL, Calluna vulgaris. DRASUB, Dracophyllum subulatum. EPAALP, Epacris alpina. CHIRUB, Chionochloa rubra. OZOVAU, Ozothamnus vauvilliersii. VERVEN, Veronica venustula. Bipartite networks for each plot are shown in Appendix 2, Figure 1- 4.

Figure 27 Network level metrics for plant-mycorrhizal networks in response to factorial warming and removal treatment $(n=8$ per treatment, 32 total). Median values for each metric are denoted by a horizontal line, boxes represent the interquartile ranges and whiskers denote the upper and lower $95 \%$ confidence intervals for each metric in response to the four treatments. All metrics were calculated with the networklevel function in the bipartite package in $\mathrm{R}$. The analyses are organised by rows of analyses pertaining to network structure and network interactions. Significant treatment effects from the Two-Way ANOVA are denoted as; $* * * \mathrm{p}<0.0005, * * \mathrm{p}<0.005, * \mathrm{p}<0.05$.

Figure 28 Trophic-level "structure" metrics for plant-mycorrhizal networks in response to factorial warming and removal treatments $(n=8$ per treatment, 32 total). Median values for each metric are denoted by a horizontal line, boxes represent the interquartile ranges and whiskers denote the upper and lower 95\% confidence intervals for each metric in response to the four treatments. All metrics were calculated with the grouplevel function in the bipartite package in R. Significant treatment effects from the Two-Way ANOVA are noted with $* * *$ if $\mathrm{p}<0.0005$, ** if $\mathrm{p}<0.005, *$ if $\mathrm{p}<0.05$. WxR, Warming and Removal factors as a significant treatment effect. 
Figure 29 Trophic-level "robustness" metrics for plant-mycorrhizal networks in response to factorial warming and removal treatments $(n=8$ per treatment, 32 total). Median values for each metric are denoted by a horizontal line, boxes represent the interquartile ranges and whiskers denote the upper and lower 95\% confidence intervals for each metric in response to the four treatments. All metrics were calculated with the grouplevel function in the bipartite package in R. Significant treatment effects from the Two-Way ANOVA are noted with $* * *$ if $\mathrm{p}<0.0005$, ** if $\mathrm{p}<0.005, *$ if $\mathrm{p}<0.05$. WxR, Warming and Removal factors as a significant treatment effect.

Figure 30 Species-level "structure" metrics for plant-mycorrhizal networks in response to factorial warming and removal treatments $(n=4-8$ per treatment, 32 total). Median values for each metric are denoted by a horizontal line, boxes represent the interquartile ranges and whiskers denote the upper and lower 95\% confidence intervals for each metric in response to the four treatments. All metrics were calculated with the specieslevel function in the bipartite package in R. Significant treatment effects from the Two-Way ANOVA are noted with $* * *$ if $\mathrm{p}$ $<0.0005, * *$ if $\mathrm{p}<0.005, *$ if $\mathrm{p}<0.05$. WxR, Warming and Removal factors as a significant treatment effect. CALVUL, Calluna vulgaris. DRASUB, Dracophyllum subulatum. EPAALP, Epacris alpina. CHIRUB, Chionochloa rubra. OZOVAU, Ozothamnus vauvilliersii. VERVEN, Veronica venustula.

Figure 31 Species-level "robustness" metrics for plant-mycorrhizal networks in response to factorial warming and removal treatments $(n=4-8$ per treatment, 32 total). Median values for each metric are denoted by a horizontal line, boxes represent the interquartile ranges and whiskers denote the upper and lower 95\% confidence intervals for each metric in response to the four treatments. All metrics were calculated with the specieslevel function in the bipartite package in R. Significant treatment effects from the Two-Way ANOVA are noted with *** if $\mathrm{p}$ $<0.0005, * *$ if $\mathrm{p}<0.005$, $*$ if $\mathrm{p}<0.05$. WxR, Warming and Removal factors as a significant treatment effect. CALVUL, Calluna vulgaris. DRASUB, Dracophyllum subulatum. EPAALP, Epacris alpina. CHIRUB, Chionochloa rubra. OZOVAU, Ozothamnus vauvilliersii. VERVEN, Veronica venustula.

Figure 32 Interaction matrix featuring modules of the ericoid mycorrhizal network Modules were identified by LPAwb+ method (Beckett, 2016), using computemodules function in the bipartite package in R. where Q $=0 \cdot 29$. Darker squares indicate stronger interactions. Red boxes delineate the seven modules. CALVUL, Calluna vulgaris. DRASUB, Dracophyllum subulatum. EPAALP, Epacris alpina. 
Figure 33 Connection (c) and participation ( $\mathrm{z}$ ) values plotted for ericoid mycorrhizal fungi OTUs in the network of ericaceous host plant species. Values calculated using computemodules and czvalues function in the bipartite package in R. Black lines indicate critical values according to Olesen et al., (2007) $\mathrm{C}$-value (Connection) $=0.62$ and Dormann and Strauss, (2014) Z-value (Participation) $=1.70$ for weighted bipartite networks. Top right quantile indicates OTUs exceeded both thresholds and are considered network "hub" species.

Figure 34 Interaction matrix featuring modules of the arbuscular mycorrhizal network Modules identified by LPAwb+ method (Beckett, 2016), using computemodules function in the bipartite package in R. Q (modularity) $=0.232$. Darker squares indicate more observed interactions. Red boxes delineate the nine modules found. CHIRUB, Chionochloa rubra. OZOVAU, Ozothamnus vauvilliersii. VERVEN, Veronica venustula.

Figure 35 Connection $(C)$ and participation $(Z)$ values for arbuscular mycorrhizal fungi OTUs in the network of arbuscular-mycorrhizal host plant species. Values calculated using computemodules and czvalues function in the bipartite package in $\mathrm{R}$. Black lines indicate critical $\mathrm{C}$ value (Connection) $=0.62$ (Olesen et al., 2007) 0.62 and Z-value $($ Participation $)=1.7$ (for bipartite networks see Dormann and Strauss (2014). Top right quantile indicates OTUs exceeded both thresholds and are considered network "hub" species. CHIRUB, Chionochloa rubra. OZOVAU, Ozothamnus vauvilliersii. VERVEN, Veronica venustula 


\section{List of Abbreviations}

$\begin{array}{lll}\text { TNP } & - & \text { Tongariro National Park (New Zealand) } \\ \text { WaRM } & - & \text { Warming and Removal in Mountains experiment. } \\ \text { OTCs } & - & \text { Open Top Chambers } \\ \text { CMN } & - & \text { Common Mycorrhizal Network } \\ \text { MF } & - & \text { Mycorrhizal Fungi } \\ \text { AM } & - & \text { Arbuscular Mycorrhizal } \\ \text { ErM } & - & \text { Ericoid Mycorrhizal } \\ \text { PIPITS } & - & \text { Automated pipeline for analyses of fungal internal transcribed } \\ \text { MRPP } & - & \text { Non-Metric Multidimensional Scaling } \\ & & \end{array}$




\section{List of Species used in this study.}

Calluna vulgaris

Chionochloa rubra

Dracophyllum subulatum

Epacris alpina

Ozothamnus vauvilliersii

Veronica venustula
- $\quad$ Common Heather

Exotic invader of alpine and subalpine regions of

TNP, introduced in 1910 and widely naturalised.

(CALVUL) Ericoid Mycorrhizal (ErM)

- $\quad$ Endemic red tussock grass.

Distribution across grasslands from Central

Volcanic Plateau to Stewart Island.

(CHIRUB) Arbuscular Mycorrhizal (AM)

- $\quad$ Monoao - Endemic woody Ericaceous shrub.

Distribution confined to Central Volcanic Plateau.

(DRASUB) Ericoid Mycorrhizal (ErM)

- $\quad$ Endemic woody Ericaceous shrub

Distribution across alpine and subalpine regions of the North Island and South Island.

(EPAALP) Ericoid Mycorrhizal (ErM)

Mountain tauhinu - Endemic bushy shrub

Distribution across alpine and subalpine regions of the North Island and South Island.

(OZOVAU) Arbuscular Mycorrhizal (AM)

- $\quad$ Endemic bushy woody shrub -

Distribution across alpine and subalpine regions of the North Island and South Island.

(VERVEN) Arbuscular Mycorrhizal (AM) 


\title{
Chapter I.
}

\section{Literature Review, Aims and Hypotheses}

\author{
Literature Review
}

\section{Climate change effects on alpine plant communities}

Climate change in alpine ecosystems has manifested through increased temperatures, nitrogen deposition rates and altered precipitation patterns (Beniston et al., 1997; Loarie et al., 2009; Kou-Giesbrecht and Menge, 2019). Air temperatures within alpine regions are projected to increase by a further $1.5-2.5^{\circ} \mathrm{C}$ compared to the global mean at the end of the 21st Century (IPCC, 2014). Alpine surface temperatures are also predicted to exhibit more dramatic increases, due to the mosaic nature of mountainous terrain (Scherrer and Körner, 2009). In parallel, alpine ecosystems are also experiencing extremes in precipitation patterns due to climate change (Pepin et al., 2005; Stoffel and Huggel, 2012). Furthermore, there is growing evidence that climate-induced alterations to hydrological cycles are increasing the frequency and scale of disturbance events in alpine environments (Cannone et al., 2007; Fatichi et al., 2015).

Consequently, the drivers of global change within alpine regions have direct impacts on the structure and functions of its biotic communities. There is strong evidence that increasing temperatures, nitrogen deposition rates, and altered hydrology are significantly impacting nutrient and phenological cycles of alpine plant communities (Mote et al., 2005; Neff et al., 2002; Bobbink et al., 2010; Wirth et al., 2011). Alpine landscapes are likely to magnify these effects as they are characterised by steep 
elevational gradients of abiotic and biotic conditions, defined as ecotones (Beckage et al., 2008). Drivers of global change are therefore shifting the spatial distributions of these ecotones, creating new tensions between the abiotic limitations of high alpine environments and the elevational rising of species (Nogués-Bravo et al., 2007; Cannone et al., 2007). Furthermore, alpine plant communities are considered to have an intrinsically high temporal lag in their responses to environmental change, due to their relatively slow growth and reproduction rates compared with plant communities of warmer climates (Alexander et al., 2018). Therefore combined with relatively high rates of global change pressures and lag in their response, alpine plant communities are considered as one of the most vulnerable to biodiversity and functional losses in the face of climate change (Klanderud, 2005; Hülber et al., 2016).

Alpine plant communities consist of species that significantly vary in their range dynamics and growth rates (Hülber et al., 2016). Current projections therefore expect alpine plant communities will experience significant species turnover in their compositions in response to climate change (Rumpf et al., 2018; Mayor et al., 2017). In parallel, it is predicted climate change will significantly alter interaction dynamics within alpine plant communities (Grabherr et al., 2010). Therefore predicting the collective response of alpine plant communities to climate change is complex (Hülber et al., 2016).

Climate shifts in alpine ecotones were initially predicted to promote species richness and resilience to invasion in the plant communities (Kullman, 2010; Theurillat et al., 2001). This conclusion was derived from analyses that found alpine plant community productivity, as measured by growth and reproduction rates, increased when the soil was 
warmed and enriched with nitrogen (Bowman et al., 1993; Beniston et al., 1997). However, the analysis by Kikvidze et al., (2005) demonstrated that the relief of these abiotic limitations impacted alpine plant communities significantly differently along temperature gradients. In summary, Kikvidze et al., (2005) found that at increased temperatures alpine plant communities at warmer study sites increased productivity, species richness and species turnover. Colder and intermediary climate study sites were found to be less productive when warmed, with lower species richness but with high turnovers in their compositions. Kikvidze et al., (2005) concluded that collectively alpine communities likely experienced a weakening of established interactions and thus a reduction in community stability. Pauchard et al., (2009) builds upon these findings and demonstrates that the relief of alpine abiotic limitations are asymmetrically exploited by invasive or dominant species, further changing species compositions and interaction dynamics. As such, alpine plant species with slower rates of growth and reproduction are predicted as the "losers" in the scenario of climate change (Rumpf et al., 2018).

Collectively, research has shown that alpine plant communities are presenting a variety of structural and functional changes to their composition in response to warming and nutrient enrichment (Rumpf et al., 2018). As alpine plant community compositions change, so do its established interaction dynamics. Significantly, interactions by plant communities with other trophic levels have shown to both mitigate, and intensify climate change effects (Brooker, 2006; Mayor et al., 2017). In light of this, Alexander et al., (2018) challenged research efforts to focus on changes in plant community interactions and their effects on ecosystem functions, rather than alterations in their compositions. 


\section{Below ground interactions of alpine plant communities}

Interaction dynamics of plant communities determine a variety of functions that facilitate the stability of the community and its wider ecosystem processes (Choler et al., 2001). A primary example of this in alpine plant communities is the development of neighbourhood microhabitats as an evolutionary approach to the abiotic limitations of alpine environments (Callaway et al., 2002). Neighbourhood effects are generated by different plant species through their interactions, facilitating aspects of nutrient cycling, soil stabilisation and pollination processes that increase the fitness of plants species within the community (Bronstein, 2009; Hegland, 2014). The long term feedback loops of facilitation effects are considered as essential to the stabilisation of the alpine plant community (Callaway et al., 2002; Wirth et al., 2011). Furthermore, neighbourhood effects have indicated to be a significant component in retaining alpine community compositions and functions under warming (Pugnaire et al., 2020).

Notably, the interactions between plants and their associated soil biota are considered to be the foremost contributors to neighbourhood effects, through the determination of decomposition and nutrient uptake rates (Hausmann and Hawkes, 2009). In turn, these interactions develop long-term feedback loops that significantly contribute to plant species fitness, nutrient cycling and thus the stabilisation of ecosystem functions (Yang et al., 2018; Lehmann et al., 2011). As such, plants-soil biota interactions are considered as the key contributors to ecosystem stabilisation, through an effect termed as "soil legacy" (Kostenko et al., 2012). Soil legacy pertains to the specific abiotic (e.g., baseline fertility, nutrient concentrations) and biotic components of the soil environment 
that cumulatively sustain ecosystem functions. Soil legacies that underly plant communities arise over time through species specific interactions, for example, Bever (1994) demonstrated that the accrual of species-specific soil pathogens limited the monodominance of strong competitors within a plant community. Additionally, preexisting plant-soil biota can influence the outcome of competition between plant species (Le Roux et al., 2018) and be a significant component in the recovery of plant communities to disturbance (Aprahamian et al., 2016; Cameron et al., 2013). Soil legacy effects resulting from plant-soil biota interactions have been demonstrated as the key determinant of community composition and resistance to invasion across a variety of plant community studies (Le Roux et al., 2018; Zuppinger-Dingley et al., 2016; Legay et al., 2018)

However, plants and their soil biota are vastly complex systems, consisting of many subset networks including pathogens, bacteria and mycorrhizal symbioses, each particular in their effect on soil legacy within their specific plant community. As such, research on changing plant communities has primarily focused on investigating networks of soil biota known to directly and consistently contribute to ecosystem functions and plant community compositions (Legay et al., 2018). The forefront of this focus is the mycorrhizal fungi of plants. Plant-mycorrhizal interactions are globally ubiquitous and foundational to nutrient cycling and plant species fitness (Ferlian et al., 2018). Therefore, as highlighted by Alexander et al., (2018), analysing interactions between host plants and their mycorrhizal fungi may provide greater insight as to how alpine plant communities and their functions are responding to drivers of climate change. 


\section{Interactions between mycorrhizal fungi and their host plants}

Mycorrhizal fungi (MF) form an association with their host plants by colonising the root tissue. Primarily, the interaction is mutually symbiotic in which the mycorrhizal fungi supplies the host plant with a more efficient uptake of water and minerals (Read and Perez-Moreno, 2003). In exchange, host plants supply their associated mycorrhizal fungi with carbohydrates from their photosynthetic processes (Smith and Read, 2010). The interaction is directly observable through the microscopic identification of mycorrhizal structures within and around plant root tissue (Smith and Read, 2010). In addition, nutrient transfer between plant and MF has been routinely tracked through the use of dual stable ${ }^{13} \mathrm{C}$ and nitrogen ${ }^{15} \mathrm{~N}$ isotope signatures (Gleixner et al., 1993; Klink et al., 2020). Notably, there is significant diversity in the nature of interactions between plants and their MF. Some MF have been identified as parasitic (Johnson and Graham, 1997), whereas, some MF taxa have proven essential to plant species fitness by facilitating the protection of plant roots against herbivory and toxicity (Babikova et al., 2013; Johnson and Gilbert, 2015; Tam, 1995).

Currently, of the six structural classes of mycorrhizal fungal symbioses recognised, arbuscular mycorrhiza (AM), ectomycorrhiza (EcM) and ericoid mycorrhiza (ErM), are the most geographically widespread (Smith and Read, 2010). AM, EcM and ErM are associated with $90 \%$ of the Earth's terrestrial plant species (Brundrett 2009; Smith and Read, 2010). Each mycorrhizal structural class forms interactions with host plants of distinct functional guilds, with very rare exceptions of dual colonisation (Smith and Read, 2010). AM fungi occupy the most diverse functional guild of MF as they form 
mycorrhizas with $72 \%$ of the world's vascular plant species (Brundrett, 2009). This is an consequence of the ancestral evolutionary mutualism between Glomeromycota fungi and early embryophytes that enabled the terrestrialization of plants (Smith and Read, 2010). Contrastingly, ErM fungi exclusively form mycorrhizas with members of the plant family Ericaceae. Both ErM and AM are classified as endomycorrhizas, whereby their colonisation of host plants involves their fungal hyphae penetrating the cells of the root tissue. Comparatively, ErM are considered to have the highest saprotrophic capabilities of the MF types, enabling significantly more efficient decomposition processes within their soil communities (Smith and Read, 2010). A comparison of the morphology of ErM and AM mycorrhizas is visualised in (Figure 1).

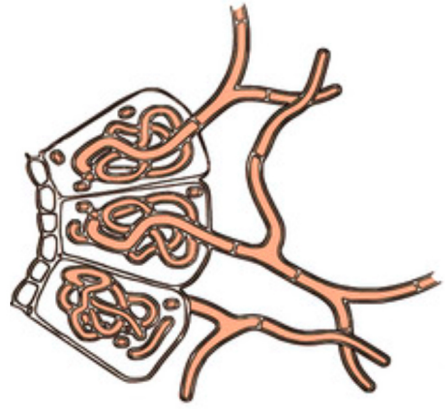

Ericoid Mycorrhiza ERM

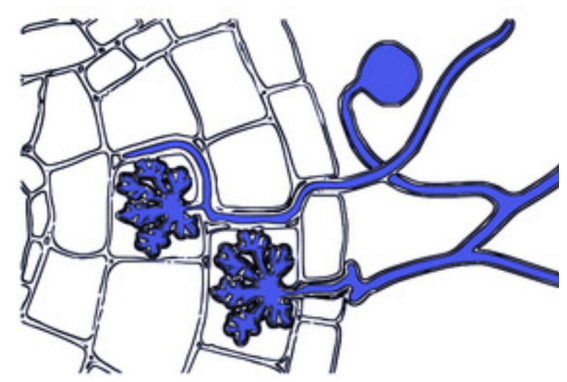

Arbuscular Mycorrhiza AM

Figure 1. (Perotto et al., 2018) Morphological comparison between intracellular root tissue colonisation of ericoid mycorrhizas and arbuscular mycorrhizas. Notably, arbuscular mycorrhizas are distinguished from their formation spherical storage organs called vesicles and sites of nutrient exchange called arbuscules.

The three MF types are considered to be associated with particular biomes where 1) $\mathrm{AM}$ interactions dominating temperate grasslands and savannahs 2) EcM dominating boreal and temperate forests and 3) ErM dominating tundra and alpine regions (Smith 
and Read, 2010). Later investigations found that abiotic factors explained $50 \%$ of the functional guild distribution of MF (Soudzilovskaia et al., 2017; Averill et al., 2019). These analyses found that temperature and soil nutrient content were the most significant explanatory variables for MF distribution. Subsequently, climate-driven decomposition gradients were identified as the primary mechanism determining global distributions of EcM and AM (Steidinger et al., 2019). Due to the differences in functional guild distributions, Bellgard and Williams, (2011) predicted that AM mycorrhizal diversity will be the least impacted by drivers of global change, whereas both EcM and ErM will be highly vulnerable to changes in plant community compositions. This conclusion is supported by Klink et al., (2020) which demonstrated that under climate warming, AMF are likely to expand their range within alpine regions due to a relief in frost limitations.

There is a growing volume of manipulative studies analysing the response of MF to agents of global change such as increased nutrient deposition (Ma et al., 2020; Allen et al., 2016), increased precipitation (Augé 2001; Cheng et al., 2016) warming (Kilpeläinen et al., 2020; Heinemeyer et al., 2004) and plant invasion (Vogelsang and Bever, 2009; Duell et al., 2016). Conversely, plant-MF interactions have also been shown to shift along a mutualist-parasitic continuum due to changes within their environmental conditions (Johnson and Graham, 1997). Currently, it is considered that due to the diversity within mycorrhizal compositions, parasitic MF are unlikely to be determinants of naturally assembled communities (Smith and Smith, 2013). Although, as outlined by Wahl and Spiegelberger (2016), the mutualist-parasitic continuum may become consequential at varying elevations within changing alpine plant communities (Figure 2). 

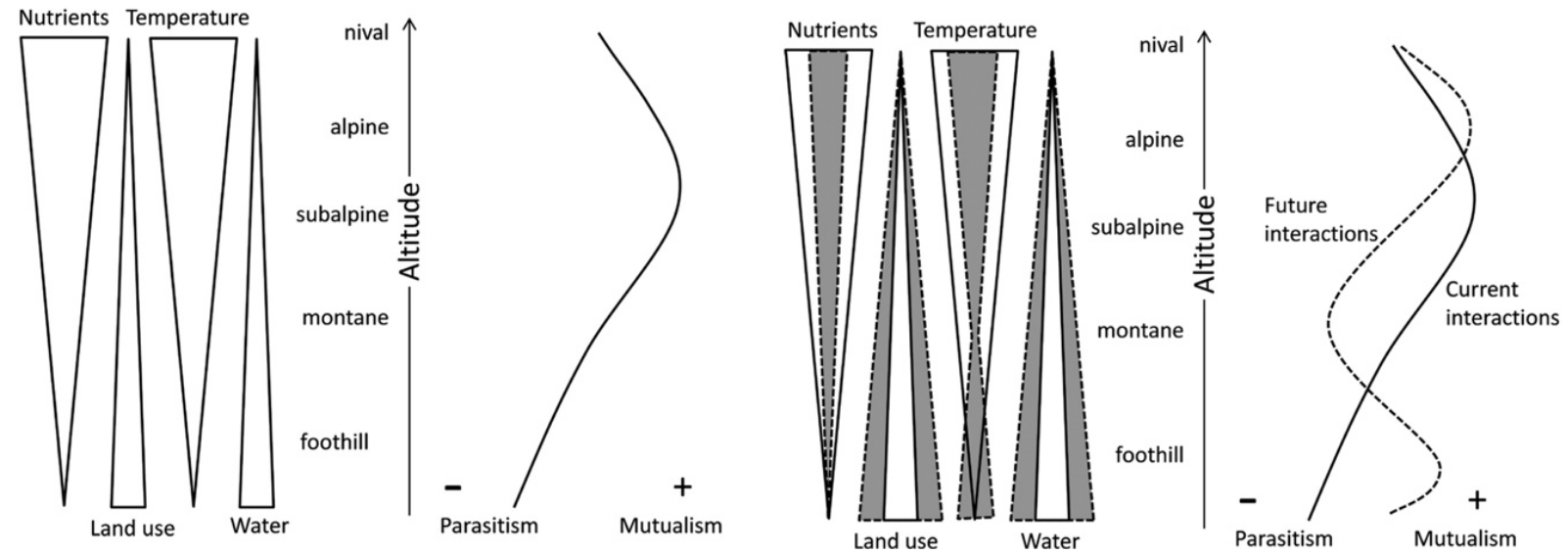

Figure 2. Wahl and Spiegelberger, (2016) demonstrate how plant-MF interactions can shift in their symbiotic nature along an elevational gradient in an alpine system due to the abiotic limitations (left). They further illustrate how nature of the plant-MF interactions may alter under increased abiotic limitations due to factors of global change (right).

Yet the majority of these studies, as examined by Ferlian et al., (2018), are limited in their conclusions as they do not measure MF responses within naturally assembled communities, nor the effects of MF responses on the plant community. Furthermore, another gap identified by Marín and Kohout, (2020) is the lack of studies of multiple MF functional guilds within one experiment. This is particularly critical to understand as MF compositions and their functional diversity have shown to be highly dependent on the plant species present within a community (Bogar and Kennedy, 2013; Rodriguez-Ramos et al., 2020). Therefore, the response of plant-MF interactions to agents of global change are more accurately analysed at the community level, in which multiple plant species and their associated MF and MF functional guilds are considered. This consideration is critical for analyses of alpine plant communities as ErM, EcM and AM interactions regularly co-occur within these regions (Soudzilovskaia et al., 2017). Furthermore, the consideration of multiple host plants is crucial as individual MF typically interact with more than one plant and plant species within their functional guild in a community, creating hyphal networks between their host plants (Bücking et al., 2016). 


\section{Plant-mycorrhizal networks}

Typically in naturally assembled plant communities, host plants interact with multiple individuals of mycorrhizal fungi (Montesinos-Navarro et al., 2012). Conversely, through the formation of hyphal networks, an individual mycorrhizal fungi interacts with multiple host plant species of the same functional guild. The formation of these networks between multiple individuals of mycorrhizal fungi and host plants are defined as common mycorrhizal networks (CMNs) (He et al., 2003; Selosse et al., 2006). Notably, CMNs frequently occur across all functional guilds of mycorrhizal types. The particular function of CMNs are highly dependent on environmental conditions and the species of host plant and MF individuals present (He et al., 2003; Barto et al., 2012). Primarily, the formation of CMNs are considered to significantly facilitate and promote the fitness of its host plant and MF participants by maintaining diverse mutualistic benefits (van Der Heijden and Horton, 2009). However, CMNs are inherently asymmetric in their benefit to their participants, due to varying roles of function and rates of nutrient transfer (Bücking et al., 2016). In particular, MF has been shown to preferentially colonise highly productive host plants over less productive hosts, forming strong feedback loops of facilitation between particular plant-MF interactions regardless of species (Zhang et al., 2010; Bücking et al., 2016). In addition, strong interactions within CMNs can promote both inter-plant and inter-fungal competition over resources, further accentuating the asymmetric benefits of a CMN on its participants (Figure 3). However, despite the complex interaction dynamics of CMNs, there are extremely few studies that consider the interactions within and between co-occuring CMNs and their effects (Marín and Kahout, 2020). 


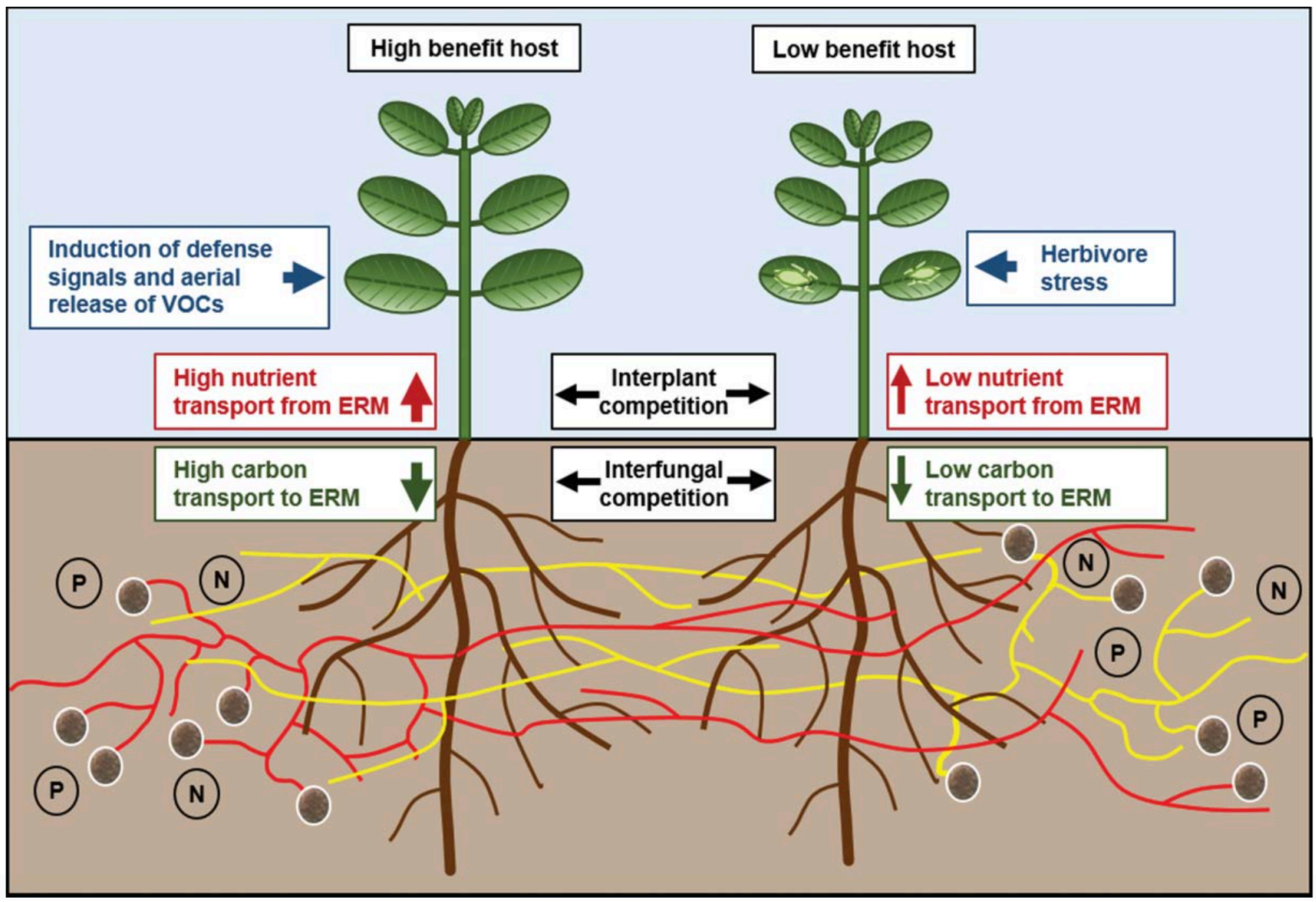

Figure 3. Bücking et al., (2016) illustrate how the formations of common mycorrhizal networks can elicit interactions that strongly and asymmetrically influence host plant and MF fitness within a naturally assembled community.

Through such strong interaction dynamics, CMNs are regarded as integral to the stability and resilience of plant communities facing agents of change (Yang et al., 2018). CMNs have been evidenced as critical in maintaining high levels of biodiversity in plant community compositions under drought (Augè, 2001; Pickles and Simard, 2017), increased temperatures (Hawkes et al., 2008; Legay et al., 2018) and increased soil nutrient concentrations (van't Padje et al., 2020; Muneer et al., 2020) CMNs have also shown to exhibit resilience in their MF and plant compositions under species loss and invasion (Anderson et al., 2010; Barto et al., 2010; Zuppinger-Dingley et al., 2016) However, the effects of $\mathrm{CMNs}$ do not consistently mediate the responses of plant 
communities or MF compositions to these agents of global change. In particular, CMNs have been evidenced to weaken in their interactions and lose diversity in their functions in their response to warming (Sun et al., 2013; Geml et al., 2015) and plant invasions (Zhang et al., 2010 Dickie et al., 2010, Grove et al., 2017; Duell et al., 2016). Furthermore, CMNs have shown to facilitate invasions and promote their effects on MF compositions (Lekberg et al., 2013; Zhang et al., 2010; Clavel et al., 2020). Additionally, as highlighted by Marín and Kahout, (2020), CMNs of different MF guilds have shown to vary in their response to drivers of change and disturbance types, implying further alterations to the functions and compositions of plant-MF communities. Consequently, maintaining diversity and network connectivity within MF compositions is regarded as a primary management strategy for upholding the stability of plant community compositions and functions (Jing et al., 2015; Rodriguez-Ramos et al., 2020).

In light of the dichotomy in the stabilisation effects of CMNs, Zobel and Öpik, (2014) propose that plant-MF networks are asymmetrically sensitive to changes at the trophic levels. The study postulates that dependent on which functions, either by MF or host plants, that are the most essential to maintaining the community's compositions, changes to that trophic level will significantly destabilise the composition of the network. Essentially, this is a further development of the driver-passenger hypothesis, where drivers are the controlling component of community composition (Hart et al., 2001). However, instead of a fixed designation of a "driver" trophic level within the network, Zobel and Öpik, (2014) propose the habitat hypothesis, whereby the "driver" is dependent on the environmental conditions present. By considering this variation in "drivers" across 
plant-mycorrhizal communities, this can highlight both consistencies and distinctions in their response to climate change effects.

Efforts to disentangle the function and structure of CMNs have been limited due to complexities in identifying its participants (Toju et al., 2014). However, recent developments in molecular methods have allowed for high resolution quantification of the abundance and diversity of mycorrhizal fungi. Specifically, Chagnon and Bainard (2014) identified that root DNA serves as a reliable proxy for mycorrhizal fungal diversity and abundance through the amplification of the internal transcribed spacer (ITS) region of the fungal rRNA gene. Since then, molecular methods have been rigorously tested to identify that the sequencing of the ITS2 region (located between 5.8S and $28 \mathrm{~S}$ subunits on the fungal rRNA gene), from plant root samples, retains the highest fidelity in mycorrhizal fungal diversity and abundance to the total sampled network (Toju, 2015). Furthermore, the development of automated pipelines and taxonomic databases specific to fungal analysis have allowed for high-throughput processing of sequence read data (Gweon et al., 2015; Nguyen et al., 2016). As such, plant-mycorrhizal communities are significantly more viable to analyse.

With the improved capacity to analyse CMNs, there are now a multitude of opportunities to further understand their role and function. As aforementioned, a worthwhile avenue for research is to examine alterations in plant-mycorrhizal interactions in response to drivers of global change. Conversely, plant invasions are of particular research interest, as invaders are a significant driver of change within CMNs (Awaydul et al., 2019) as well as within alpine plant communities (Pauchard et al., 2009). 


\section{Invasions of alpine plant mycorrhizal communities}

Alpine plant communities were predicted as resilient to invasion due to their relative isolation and harsh climatic conditions (Grime, 1977; Callaway et al., 2002; McDougall et al., 2011). The combination of abiotic limitations within alpine regions has resulted in its invasion resilience to be attributed to "abiotic resistance" (Alpert et al., 2000). Conversely, alpine regions were considered to inherently possess low levels of "biotic resistance", due to relatively low levels of biodiversity and comparatively high proportions of facilitative neighbourhood interactions.(Pauchard et al., 2009). Therefore the relief of abiotic limitations resulting from climate change, primarily, increased temperatures and soil nutrient availability, are considered to be critical threats to the invasion resilience of alpine communities (McDougall et al., 2011; Trivedi et al., 2008). Furthermore, the frequency and diversity of invasion pathways are increasing in alpine regions due to the intensification of land use and densification of tourism infrastructure (Pauchard et al., 2009). Additionally, as alterations to hydrological cycles are increasing the frequency and scale of disturbance events, so are the opportunities for invader establishment within alpine regions (Cannone et al., 2007; Fatichi et al., 2015).

The synergistic relationship between plant invasions and climate change indicate that alpine regions are critically vulnerable to significant alterations in their composition and functions (Brook et al., 2008). Presently, there is strong evidence of climate warming in combination with plant invasions significantly altering alpine ecosystem functions, such as, pollination (Giejsztowt et al., 2020) seed dispersal (Hoyle et al., 2013) and nutrient cycling (He et al., 2003; Xu et al., 2011). However, aside from a few studies 
reporting reductions in alpine MF diversity (Geml et al., 2015; Grove et al., 2017), there remains little research as to how the independent and combined effects of plant invasions and warming impacts alpine plant-MF communities and functions. As CMNs have also shown evidence of influencing invasion resilience in plant communities, Ferlian et al., (2018), advocates for research to focus on their response to the independent and combined effects of warming and plant invasions.

The degree to which mycorrhizal networks influence invasion resilience in plant communities is highly debated (Bunn et al., 2015). Primarily, there is no evidence of naturally assembled MF compositions that exhibit host selectivity to a degree that significantly and consistently excludes an invasive species (Richardson et al., 2007). Moreover, invasive species have shown to have a higher propensity for forming novel and generalist mycorrhizal interactions (Orlovich and Cairney, 2004; Nuñez and Dickie, 2014; Moora et al., 2011). Furthermore, there is evidence of native MF facilitating invader plants species with strong feedback loops (Lekberg et al., 2013; Zhang et al., 2010; Allen et al., 2020). However, there is still strong evidence of mycorrhizal composition being an essential component to preventing the mono-dominance of invaders. For example, Barto et al., (2010) found that a naturally assembled MF community significantly ameliorated the allelopathic effects of an invader on the native plants. Additionally, both highly diverse and naturally assembled MF communities were determined as the primary soil legacy effect that determined high invasion resilience (Le Roux et al., 2018). 
In the face of the conflicting evidence as to how influential mycorrhizal networks are on plant invasions, two hypotheses have been developed, the "degraded" and "enhanced" mutualism hypotheses (Bunn et al., 2015). Both hypotheses predict that invasive plants species interact significantly differently with MF than the native plant community. Furthermore, these hypotheses assume host plants are always the "drivers" of the MF compositions under the Zobel and Öpik, (2014) habitat hypothesis.

The degraded mutualism hypothesis proposes that highly influential invaders suppress the mycorrhizal interactions of native plants. Primarily this suppression is either a result of the invader being non-mycorrhizal or less colonised by the native MF in the soil (van der Putten et al., 2007; Vogelsang and Bever, 2009). This hypothesis is strongly supported by evidence of comparatively high rates of allelopathy by invaders (Lankau, 2010; Stinson et al., 2006) as well as the significant dominance of co-invader mycorrhiza in native plant communities (Dickie et al., 2010; Badano et al., 2007).

Conversely, the enhanced mutualism hypothesis suggests that invasive plant species, due to their capacity in forming generalist and novel mycorrhizal interactions, enjoy more beneficial MF interactions than in their native range (Reinhart and Callaway, 2006; Richardson et al., 2007). Consequently, it is then expected that the native mycorrhiza that form associations with the invader will increase significantly in their proportional abundance, and promote a positive feedback loop with the invader (Zhang et al., 2010). This hypothesis has not been tested for within a plant mycorrhizal community, due to the aforementioned limitations in identifying fungal diversity (Bunn et al., 2015). 
Both hypotheses were tested by Bunn et al., (2015) in a meta analysis of studies that recorded invasion effects on mycorrhizal communities. The analysis concluded that there was no consistent support of one hypothesis over another. However, notably a study by Allen et al., (2020), found that plant invaders also have a comparatively stronger direct and indirect interactions, both mutualistic and antagonistic, bridging the gap of these two hypotheses. However, evidence is still sparse on the account that many studies are focused on a few, high-profile invaders (Bunn et al., 2015).

Most of the studies within the meta-analyses did not compare mycorrhizal compositions between native plant species and the invader, but solely focused on the MF interactions by the invasive species (Bunn et al., 2015). However, the meta-analysis confirmed that MF compositions are best predicted by the host plant species they were associated with (Bunn et al., 2015). Although, this conclusion is limited by the small number of replicate samples taken across MF community analyses (Chagnon, 2016). The scarcity of data comparing MF compositions between native and invader host plant species is attributed to the limited molecular methodology available to the studies (Chagnon and Bainard, 2014). Furthermore, Bunn et al., (2015) identifies that while MF diversity analyses are insightful, they are limited in their assumptions. Particularly, diversity analyses are insensitive to changes in the strength of MF interactions, or the sharing of interaction partners which have been identified as critical components of invasion success (Allen et al., 2020; Awaydul et al., 2019). Therefore, to detect the effect of invasion on plant-mycorrhizal networks, and the role of mycorrhizal networks in invasion resilience, a more insightful analytical approach is essential. 
Network Analysis

With the newly accessible opportunities to study drivers of change within plantmycorrhizal communities, Chagnon et al., (2014) and Toju et al., (2014), advocate for a shift towards a network analysis approach. Network analysis is a toolkit of statistical techniques that evaluate the interactions between a network's participants. In the context of community ecology, network "participants" are typically defined by species, where the interactions are trophic or mutualistic dynamics between two levels. Two level networks are defined as "bipartite" networks (Figure 4, Dormann et al., 2009). Most ecological bipartite networks are asymmetric in nature whereby one level has a greater diversity of species (Rooney et al., 2006). Primary examples of bipartite networks are communities of plant-pollinator, predator-prey and plant-mycorrhizal interactions (Dormann et al., 2009).

The advantage of a network approach is that it considers how species independently and cumulatively contribute to the function and structure of a community (Dormann et al., 2017). In a bipartite network, the "function" of a species is defined by the strength, diversity and topology of its interactions with the other trophic level (Dormann et al., 2009). Comparatively, diversity analyses are insensitive to these components of function (Blüthgen, 2010). Instead, diversity analyses operate under the diversity-beget-stability hypothesis (McCann, 2000). Under this assumption, the taxonomic richness of interactions by a species is the indicator of both its function in the community, as well as its robustness against diversity loss (Pocock et al., 2012). Network analyses develop this concept further and consider species' function and robustness in relation to one another. This approach can therefore decipher the particular role and 
influence species' have on the network composition. Furthermore, the network approach can identify species that occupy similar or distinct functional roles. Identifying these properties can provide critical information for conservation management strategies and further inform on the effects of global change drivers on a community (Burkle and Alarcon, 2011)..

\section{Higher Trophic Level (i.e., Predators, Pollinators, Mycorrhizal Fungi)}

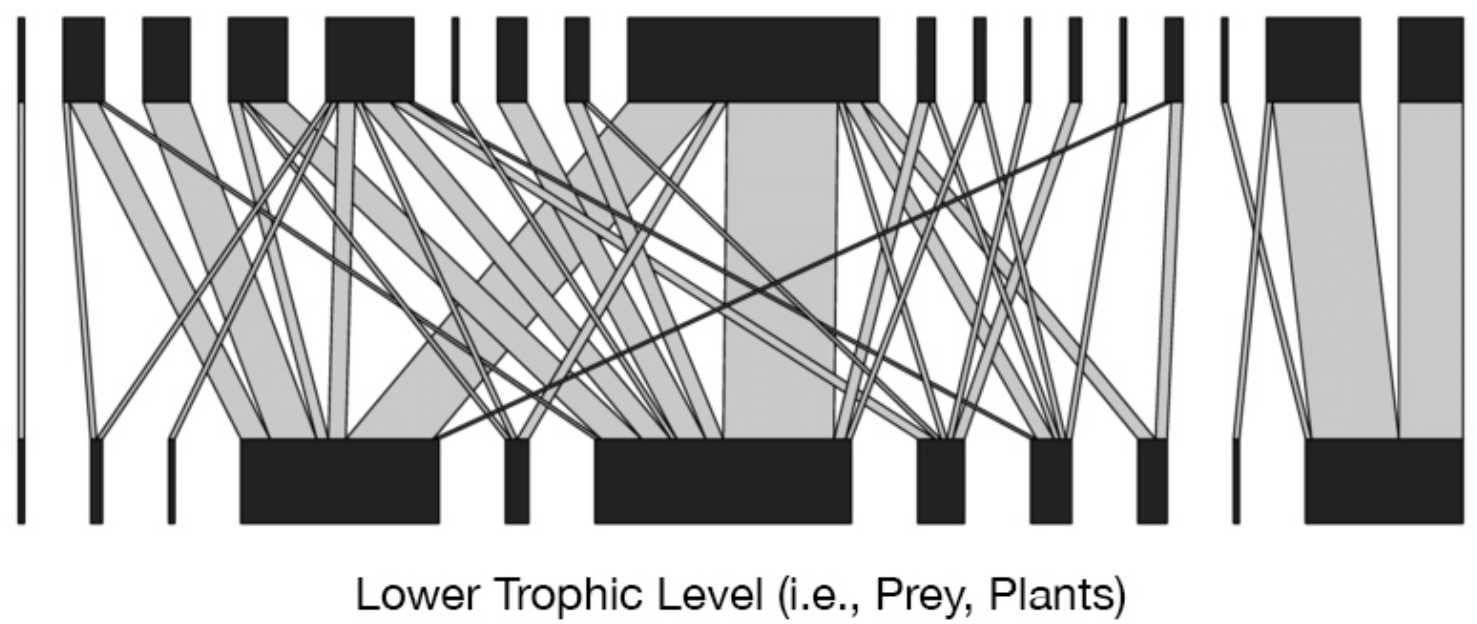

Figure 4. Dorman et al., (2009) demonstrate bipartite interaction networks constructed with the plotweb function from the bipartite package in R. "Lower Trophic level" species (interaction partners), are connected to the species the "Higher Trophic level" (source of interaction). The width of the bar for each species (i.e., node) is proportional to the relative abundance of interactions it has, and acts as a proxy for the relative influence a species exerts on the network (i.e., its centrality). The width of each link is proportional to the relative strength of the interaction, typically quantified as the number of interactions observed between the partners.

To identify properties of network structure, robustness and species' influence, Dormann et al., (2009) advises that network analyses should be approached at three distinct levels; the whole network-level, the trophic-level and the species-level. At the whole network-level, "connectivity" is the fundamental component to measure as an indicator of a network's structure and robustness. Connectivity metrics measure the 
degree to which the trophic levels interact with one another (Blüthgen, 2010). Network connectance is at its highest when all species of a trophic level strongly interact with all species of the other (Bersier et al., 2002). A key component of network connectivity is the extent of "nestedness" structure, which determines the degree to which specialists are connected to the network by a species that also interact with generalists (Ulrich et al., 2009). The prominence of nestedness structure can also indicate whether network connectivity is dependent on particular "hub" species (Brualdi and Sanderson, 1999; Ulrich et al., 2009). Most mutualist networks are highly nested (James et al., 2012), however the prevalence of nestedness within plant-mycorrhizal networks has been reported as highly variable (Toju et al., 2014; Montesinos-Navarro et al., 2012). Collectively, by calculating several facets of a network's connectivity, this illuminates aspects of its structure and robustness.

At the trophic-level, Dormann et al., (2009) recommends a variety of metrics that describe how the composition of species' functions at each trophic level contribute to the network's structure and robustness. The particular advantage of these metrics are in their ability to describe how the trophic levels independently contribute to the network's connectivity, functional redundancy and functional diversity. The properties of network functional redundancy and functional diversity are derived from community niche theory (Blüthgen and Klein, 2011). Functional redundancy arises in a network when species share interaction partners. This overlap contributes to network stability as the interaction (i.e., the network function, such as pollination, predation or symbioses), is likely to be retained in the event of species loss (Biggs et al., 2020). Functional redundancy was 
identified in a meta-analysis by Biggs et al., (2020) to be the key component retaining mutualist community functions, particularly pollination, within experiments on naturally assembled communities. Comparatively, functional diversity arises in a network when species exhibit dissimilarities in their interaction topologies and strengths. This distinction between species' functions contributes to network stability, as in the event of species loss, the members of the interacting trophic level are likely to be asymmetrically affected (Petchy and Gaston, 2006). A high occurrence of both properties within network architecture are considered as insightful proxies of community function and stability, as described in Figure 5 as "functional complementarity" (Blüthgen and Klein, 2011).

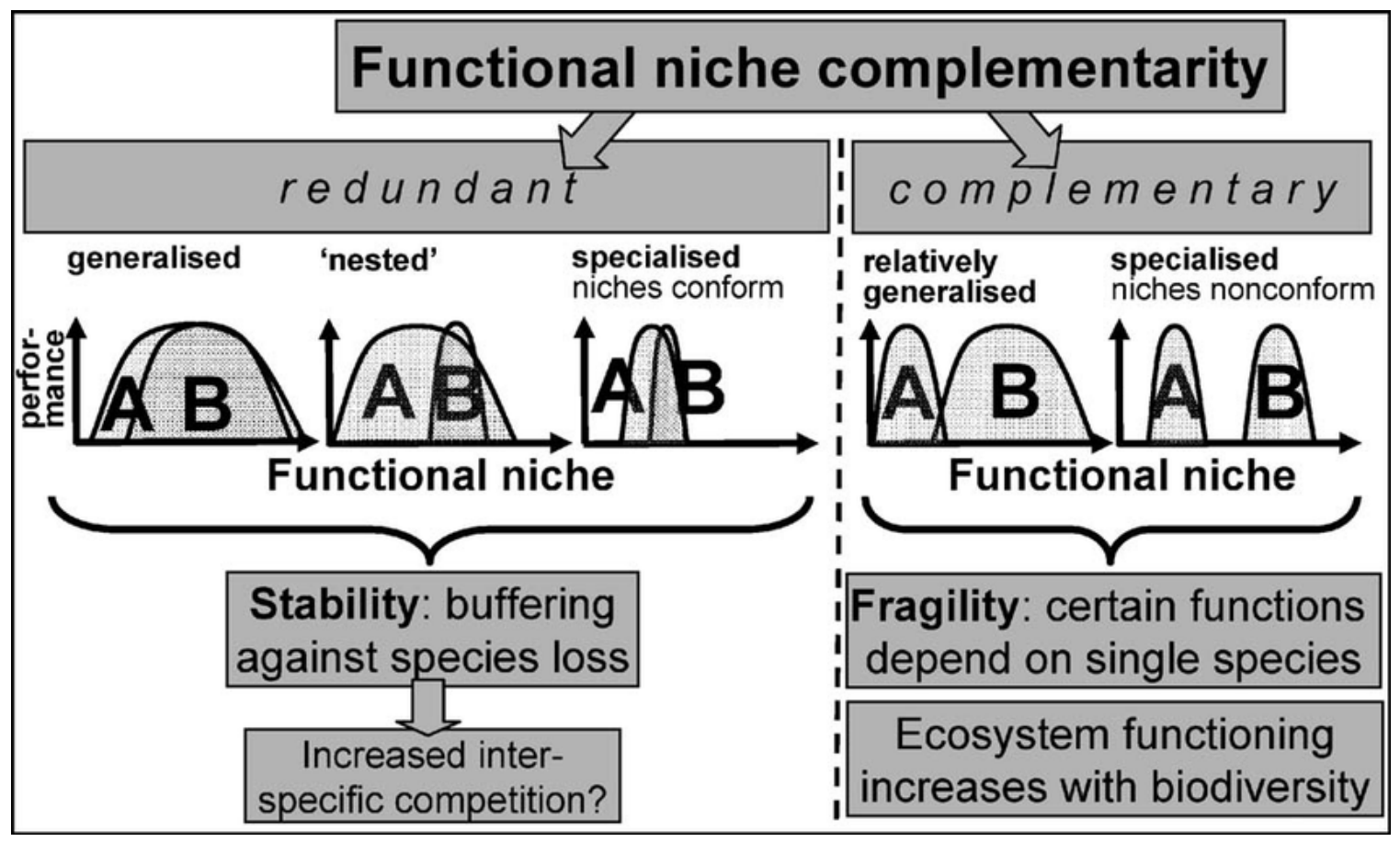

Figure 5. Blüthgen and Klein, (2011) demonstrate how the diversity of interactions exhibited by network participants occupy niche space, characterising their function. "Functional redundancy" occurs where participants A and B mostly overlap in their function role, meaning that there is significant similarity in the interactions they exhibit. Complementary functions arise when species A and B are dissimilar in their topology of interactions, representative of "functional diversity". The higher degree of both measures in a network, the higher "functional complementarity" and robustness of the network. 
Species-level analyses highlight how influential certain species are on their interaction partners and the overall network structure. Particularly, this aspect of the analysis, determines species influence through metrics calculating their proportional interaction strength, generality of interactions and the number of specialist interaction partners. This quantification of influence is characterised as a species' "centrality" in the network. Changes to species centrality indicate changes in overall network function. In particular, decentralisation quantifies the loss of influence a species has on a network, whereby its elimination from the network will manifest in comparatively fewer losses in the diversity of interaction partners and functions within the network (Lai et al., 2012). Importantly, this aspect of network analysis can highlight how species within a community are responding to drivers of change (Albrecht et al., 2014; Peay, 2016). In particular, network analyses have been utilised to identify mechanisms of successful plant invasions. As diversity analyses have previously recognised, network analyses by Moora et al., (2011) and Devoto et al., (2012) have also confirmed invaders are highly influential within a network on account of their generalist nature. However, network analyses also reveals that the success of plant invaders, as exemplified by Allen et al., (2020), are a result of their comparatively stronger interactions, both mutualistic and antagonistic, regardless of their biomass and the diversity of interaction partners. By considering species centrality alongside other measures of trophic level functions and network connectivity, this can significantly contribute to knowledge of the community functions and robustness and in turn aid conservation management strategies. 
Network analysis can also be used to illuminate other important functional characteristics within the community. In particular, Sheykhali et al., (2020) highlights how the computation of network "modularity" can reveal how significant properties such as interaction plasticity, generality and specialism are to a community's structure (Sheykhali et al., 2020). The primary limitation in the network approach is a result of the inherent restrictions of sampling ecological communities. Essentially, within experimental designs, it is rarely viable to sample interactions across multiple trophic levels to a consistent degree. Therefore, network analyses are utilising datasets that are inherently temporal and spatial subsets of the true interaction network within a community (Dormann et al., 2009). Consequently, implicit sampling bias may manifest to show false significant effects within network analysis metrics (Blüthgen, 2010). Chagnon et al., (2014) demonstrates the potential of detecting false significant effects within a network analysis with the plant-mycorrhizal network data sampled by Toju et al., (2014). To address this matter, Chagnon, (2016) insists that ecological interaction network analysis studies utilise rigorous testing of null model networks derived from the original sample data. Furthermore, measures of species' abundance, functional traits, and environmental conditions are recommended to strengthen the validity of conclusions made from significant network analyses results (Chagnon, 2016; Dormann et al., 2017). Finally, Dormann et al., (2017) highlights the need for experimental designs to utilise replicate networks and manipulative treatments to ensure that the structure of the network and its species are consistently responding to the effects being tested. 


\section{WaRM Experiments}

In light of the significant effects of warming and species invasion on plantmycorrhizal networks and alpine plant communities, WaRM (Warming and Removal in Mountains) experimental design provides a means for sampling replicate networks for analysis. WaRM is a coordinated project developed to investigate warming and dominant species as drivers of change on alpine plant communities. The experimental design combines simulated warming and dominant plant species removal by applying a factorial design at two elevations, separated by $500 \mathrm{~m}$. The project has 10 elevation research sites in 10 countries, including one in New Zealand's Tongariro National Park (TNP). Studies by Hendershot et al., (2017), Jing et al., (2015), Mayor et al., 2017, Read et al., (2018), and Okano and Bret-Harte, (2015) have all utilised the WaRM design in plant community studies as depicted in Figure 6.

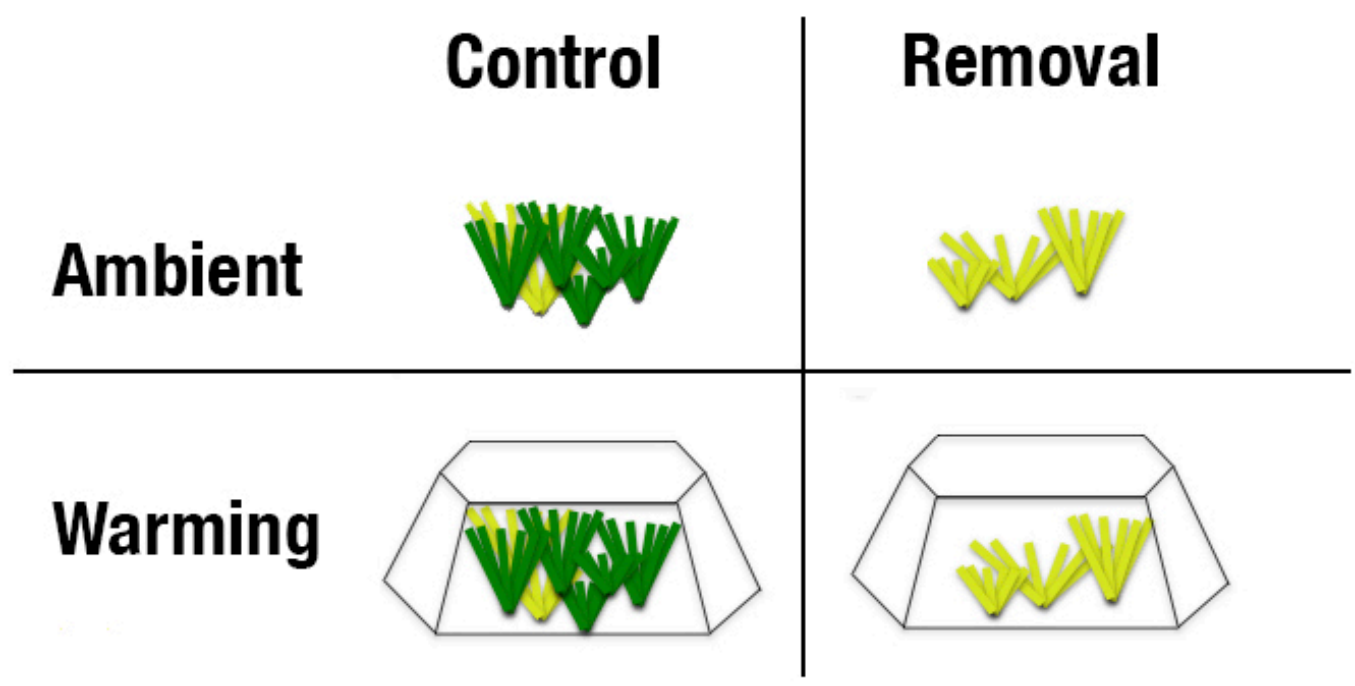

Figure 6. Diagram of the warming and dominant species removal (WaRM) experimental design. Green "plants" represent the removal of dominant plants. Warming plots demonstrate the application of Open-Top Chambers that passively warm the plots by $\sim 1.73^{\circ} \mathrm{C}$ higher than the controls. The factorial design resulting in 4 treatments. These treatment are ambient-control, ambient-removal, warm-control, warm-removal. 
The WaRM design creates warming simulations using transparent hexagonal open-top chambers (OTCs) with an interior area diameter of $2.3 \mathrm{~m}^{2}$ to passively warm plots. OTCs are commonly used in climate change experiments in remote locations to raise soil and air temperatures, using a green-housing effect (Marion et al., 1997). To confirm their effectiveness in alpine regions, Zhang et al., (2014a) tested Open-Top Chambers, against other passive heating methods, summarising that they produce the most cost-effective and consistent method of warming plots by $2^{\circ} \mathrm{C}$. Therefore OTC warming simulations align with the increases in the surface temperature of alpine regions expected at the end of the 21 st century (IPCC, 2014). OTCs also proved to cause the minimal amount of disturbance to insect visitation, soil moisture, herbivory, pollination and gas exchange.

The "removal" component of the WaRM design corresponds to the removal of a dominant plant species at the site scale. This is to determine the impact dominant plant species have on an array of community properties. Species removal experiments are particularly useful as they can be utilised within naturally assembled communities, whereas the complexity of species interactions are difficult to recreate in artificial communities (McLaren and Turkington, 2011). Furthermore, removal experiments outperform other experimental approaches when analysing the response of community functions to nonrandom extinctions, changes in the natural abundance of species, and complex interspecific interactions (Díaz et al., 2003). In addition, removal treatments are considered essential for testing invasion effects on plant communities (Guido and Pillar, 2017; Anderson et al., 2010). 
Although the WaRM experimental design has not been utilised within studies of plant-mycorrhizal communities, warming and removal treatments have independently shown to elicit significant effects on plant-mycorrhizal interactions. Specifically, manipulative warming has been evidenced to significantly alter MF compositions within plant communities from a variety of regions (Deslippe et al., 2012; Heinemeyer et al., 2004). Particularly, manipulative warming has shown to reduce the taxonomic diversity within MF compositions in alpine regions, (Sun et al., 2013; Zhang et al., 2014a; Geml et al., 2015). Conversely, species removal treatments have revealed a range of responses within MF compositions. In particular, the removal of an invasive species has shown to increase MF diversity (Dickie et al., 2010, Grove et al., 2017; Duell et al., 2016), reduce MF diversity (Stinson et al., 2006; Anderson et al., 2010) and elicit no change (Marshall et al., 2011).

Yet, there are few studies that analyse the consistency of either warming or invasive species effects on naturally assembled plant-MF communities by using replicate sampling methods (Deslippe et al., 2012; Zhang et al., 2014; Geml et al., 2015). Furthermore, despite evidence of warming promoting the influence and dominance of invasive species within alpine communities, few studies have examined the interaction of these effects on plant-mycorrhizal communities (Duell et al., 2016). Therefore there is a significant benefit in utilising the WaRM experimental design to determine the consistent responses of the alpine plant-mycorrhizal networks to warming and the presence of an invasive species. 


\section{Statement of Study's Contribution, Aims and Hypotheses}

\section{Contribution}

In light of the high rates of climate change and plant invasions in New Zealand's alpine regions, I will utilise the WaRM experiment in TNP alongside diversity and network analyses to determine the independent and interactive effects of warming and the presence of an invasive plant species on an alpine plant-mycorrhizal community.

\section{Aims and Hypotheses}

In Chapter 2, I aim to understand how the mycorrhizal fungal community of native alpine plants change in composition and diversity in response to two major drivers of global environmental change, warming and invasion.

I ask: How does warming and the presence of an invasive plant species independently and interactively impact the composition of mycorrhizal communities associated with native and an invasive plant species?

I hypothesise: Warming will reduce the diversity and alter the composition of the mycorrhizal fungal community associated with native plants. I predict that the removal of invasive ericaceous $C$. vulgaris will reduce the diversity of mycorrhizal fungi associated with host plants that form ericoid mycorrhizas, but promote the diversity of arbuscular mycorrhizal fungi associated with native host plants that form arbuscular mycorrhizas. I

predict that $C$. vulgaris will be generalist in its interactions and host a high diversity of ericoid mycorrhizal fungi relative to native host plant species. 
In Chapter 3, I aim to reveal how the architecture of plant-mycorrhizal networks change in response to warming and invasion.

I ask: How does warming and the presence of an invasive plant species independently and interactively effect the structure and robustness of alpine plantmycorrhizal networks?

In addition, I ask: Do the responses of ericoid and arbuscular mycorrhizal networks to warming and the presence of an invasive plant differ?

I hypothesise: Warming will independently reduce the strength and diversity of interactions of native plants with their mycorrhizal fungi. I predict that the removal of invasive $C$. vulgaris will reduce the diversity and robustness of the common ericoid mycorrhizal network, but increase the diversity and robustness of the common arbuscular mycorrhizal network. I predict $C$. vulgaris will act as a generalist with a strong influence on the structure and robustness of the plant-mycorrhizal network. 


\title{
Chapter II.
}

\section{The effect of warming and invasive species removal on a ericoid and arbuscular mycorrhizal fungi community structure in alpine tussock grassland.}

\author{
Introduction
}

Anthropogenic climate change effects and species invasions are particularly acute in alpine ecosystems (Grabherr et al., 2010). New Zealand alpine regions are experiencing warming, drying and species invasion at higher than global mean rates (IPCC, 2014). As New Zealand's alpine plant communities are unique globally, with high rates of local endemism, it is concerning that warming and plant invasions have also shown to independently and interactively alter plant community compositions (Cannone et al., 2007). Globally, alpine plant communities have consistently shown declines in nutrient cycling efficiency and reduced resilience to invasion in response to warming. (Zuppinger-Dingley et al., 2016; Klanderud, 2008)

Manipulative warming studies have also indicated significant changes in plantfungal community structure and diversity (Zhang et al., 2014a; Bret-Harte et al., 2004). Plant-fungal interactions, particularly mycorrhizal symbioses, are foundational to the function of alpine plant communities. Therefore detecting changes in mycorrhizal fungal compositions can illuminate the impact of warming on alpine plant community stability and function. An investigation by Geml et al., (2015) demonstrated that warming significantly reduced the richness of ericoid and ectomycorrhizal fungi within a naturally assembled arctic plant community. However, as obligate symbionts, mycorrhizal fungal compositions are highly determined by the species composition of their host plants 
(Chagnon et al., 2020). Therefore their response to environmental effects maybe significantly mediated by the response of their host plants (Bogar and Kennedy, 2013; Zobel and Öpik, 2014). As such, warming effects may manifest in a diverse range of responses within a plant-mycorrhizal community. To disentangle how these effects may manifest within a community, mycorrhizal fungal compositions should be analysed across host plant species, particularly including dominant species, invasive species and those associated with different mycorrhizal guilds.

Dominant plant species exert disproportional effects on plant-below ground community structure, especially when the dominant species is invasive (Zhang et al., 2010; Le Roux et al., 2018). A meta-analysis by Grove et al., (2017) revealed that plant invaders consistently decreased the abundance and altered the mycorrhizal fungal composition of a community, with minimal impact on the taxonomic richness of mycorrhizal fungi, indicating a turnover in the MF taxa present. Dickie et al., (2010) also demonstrated that an invader promoted their own associated mycorrhizal fungi through co-invasion, which permanently altered the composition of a plant mycorrhizal community. Duell et al., (2016) additionally concluded that increases in temperature may exacerbate the successful invasion and dominance of exotic plant-mycorrhizal symbioses. Notably, facilitative effects between warming and the dominance of invader C. vulgaris on plant-pollinator interactions has also been detected within Tongariro National Park, New Zealand (Giejztowt et al., 2020).

As warming and species invasions significantly impact plant-below ground community compositions, it is also critical to consider how these effects may manifest 
among communities of mixed plant-mycorrhizal guilds, and whether the responses of MF differ between guilds (Marín and Kahout, 2020). It is particularly interesting to consider whether an invader may promote the mycorrhizal interactions of other host plants species within its own common mycorrhizal network (CMN), and how these effects may impact another co-occuring CMN of a different mycorrhizal guild. Currently, there are two hypotheses as to how invasive plant species may impact the mycorrhizal communities of native host plants, the "degraded" and "enhanced" mutualism hypotheses (Bunn et al., 2015). The degraded mutualism hypothesis predicts that non-mycorrhizal invaders suppress the mycorrhizal interactions of native host plants (Vogelsang and Bever, 2009). Conversely, the enhanced mutualism hypothesis predicts invaders have more beneficial interactions with mutualists in their exotic range, and therefore these associated mutualists, increase in their proportional abundance. The enhanced mutualism hypothesis has not been tested for within a plant-mycorrhizal community. Additionally, neither of the hypotheses have been tested in a community of two co-occuring mycorrhizal functional guilds.

In this chapter, I analyse how the composition of the mycorrhizal fungal community of a native alpine tussock grassland responds to warming and the removal of a dominant and invasive species, Calluna vulgaris (Common Heather). I sampled the roots of the six most abundant plant species from the 32 plots of the WaRM experiment in Tongariro National Park. From these samples, I extracted DNA, PCR amplified and sequenced the fungal genes. I assessed the fungal taxonomic composition and diversity in response to the WaRM treatments and host plant species. I tested the following 
hypotheses; 1) warming will reduce and alter the diversity in the composition of mycorrhizal fungi associated with native plants; 2) the removal of $C$. vulgaris will reduce the diversity of mycorrhizal fungi associated with host plants that form ericoid mycorrhizas. In turn, based upon the degraded mutualism hypothesis, I predict the removal of C. vulgaris will promote diversity within host plants that form arbuscular mycorrhizas. Finally, given its role as an invasive species, as a habitat generalist with broad edaphic range, and as a generalist with generalist pollinators (Giejztowt et al., 2020), I predict that C. vulgaris will act as a mycorrhiza generalist, and host a high diversity of ericoid mycorrhizal fungi comparative to native host plant species and thus be comparatively less impacted by warming than the native species (Orlovich and Cairney, 2004). 
Methods

\section{Site Description}

The study site is located in Tongariro National Park (TNP), New Zealand (39 $17^{\prime}$ 36.7836" S, $175^{\circ} 43^{\prime} 35.3316^{\prime \prime}$ E) (Figure 7). TNP is a UNESCO World Heritage Site that encompasses three large andesite volcanoes and hosts alpine tussock plant communities with high levels of endemism (Chapman and Bannister, 1990; Rogers and Leathwick, 1996). The experimental study site is positioned in Rangipo desert, on the eastern side of TNP and predominantly consists of dark sandy-loam and loamy-sand soils. The site experiences frequent temperature inversions, typical of alpine flats, with a mean annual air temperature of $9^{\circ} \mathrm{C}$ and 100 days of ground frosts per year. (Smale, 1990; Giejsztowt et al., 2020). The climate of TNP is also changing at higher than the global mean rates for alpine regions (Chappell, 2015). In comparison to 50 years ago, TNP is currently $1.5^{\circ} \mathrm{C}$ warmer and receives 5mm less precipitation per year (IPCC, 2014).

The native plant community of Rangipo desert is a tussock grassland with a subcanopy of woody evergreen shrubs and herbs. Chionochloa rubra is the dominant tussock-forming grass, which is interspersed with mostly Ericaceous shrubs, in addition to arbuscular mycorrhizal shrubs, grasses and herbs. Plant communities across TNP have been invaded by European heather, Calluna vulgaris (Ericaceae). C. vulgaris was intentionally introduced to the park in 1912 (Keesing, 1995, Rogers and Leathwick, 1996) and spread rapidly to its modern extent of approximately $50-60 \%$ of the park (Peterson et al., 2020). Invasion by $C$. vulgaris is considered the primary driver of decline 
of native and endemic plant species. C. vulgaris has become the dominant plant species visible on the landscape, outcompeting the previously dominant endemic species Chionochloa rubra and Dracophyllum subulatum (Smale, 1990; Rogers and Leathwick, 1996). The aboveground transition from C. rubra to C. vulgaris dominance, is likely to be accompanied by a change in of soil biota composition. Specifically, there is likely to be a switch from the dominance of arbuscular mycorrhizal fungi (AMF) to ericoid mycorrhizal fungi (ErMF). In 2009, the Department of Conservation initiated a biocontrol programme to control C. vulgaris in the Park. The biocontrol agent, Lochmaea suturalis (Heather beetle) has established in some areas but its range is not yet widespread in TNP. 


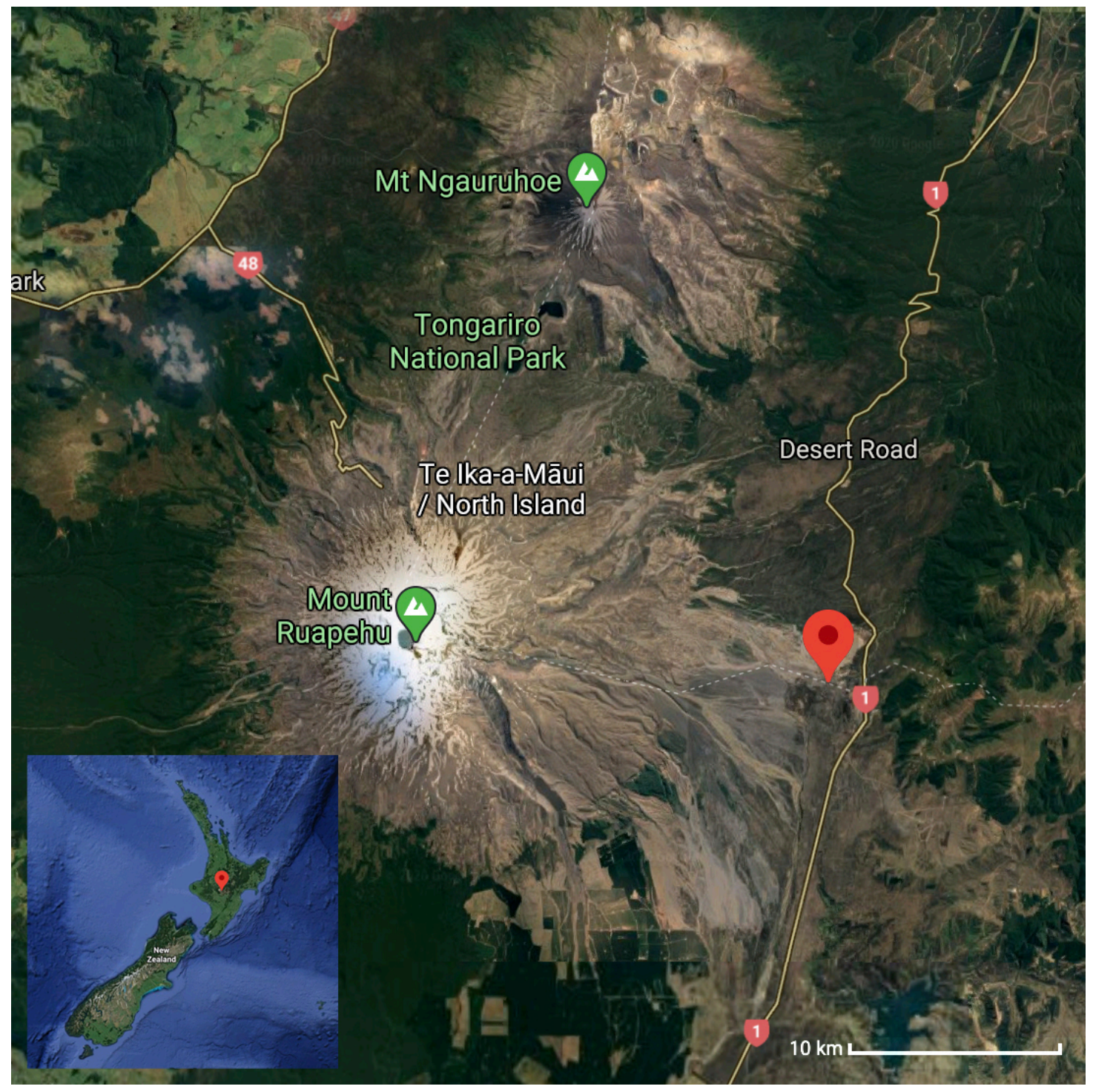

Figure 7. Satellite Image (Google Earth, earth.google.com/web) of Tongariro National Park illustrating the location of the WaRM experimental site. The site is located at $\sim 1000$ m elevation on the Rangipo desert on the eastern leeward side of Mount Ruapehu, where $C$. vulgaris is still expanding in its spatial range. 


\section{Experimental Design}

The experimental treatment consists of a $2 \times 2$ factorial combination of warming and dominant species removal ( 8 replicates per treatment, $n=32$ plots). The study is part of a globally distributed network of experiments called WaRM (Warming and Removal in Mountains). This experimental design is currently active in 9 other mountain regions globally (Okano and Bret-Harte, 2015). WaRM uses transparent hexagonal open-top chambers (OTCs) with an interior area diameter of $2.3 \mathrm{~m}^{2}$ to passively warm plots. OTCs are commonly used in climate change experiments in remote locations, to raise soil and air temperatures with minimal disturbance (Marion et al., 1997). To confirm the warming treatment effect, the plots were installed with temperature loggers (iButton ${ }^{\circledR}$, Maxim Integrated) $5 \mathrm{~cm}$ above the soil surface in all plots. Yearly mean air temperatures in OTCs were on average $1.73^{\circ} \mathrm{C}$ higher than control plots. OTCs were installed in March 2015, therefore a 4-year treatment effect was in place at the time of sampling in February 2019.

The dominant species removed in the New Zealand WaRM experiment is invasive C. vulgaris. C. vulgaris removal is maintained by clipping above ground biomass of plants and weeding seedlings, as this can be accomplished with minimal soil disturbance. Finally, the warming and dominant species removal treatments were crossed, to be applied to the 32 plots factorially. This allowed for the examination of their independent and interactive effects on plant-mycorrhizal fungal communities, where 8 plots are representative of each treatment (Figure 8). 


\section{Control $\quad$ Removal}

\section{Ambient

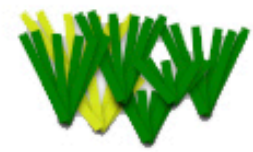

Warming
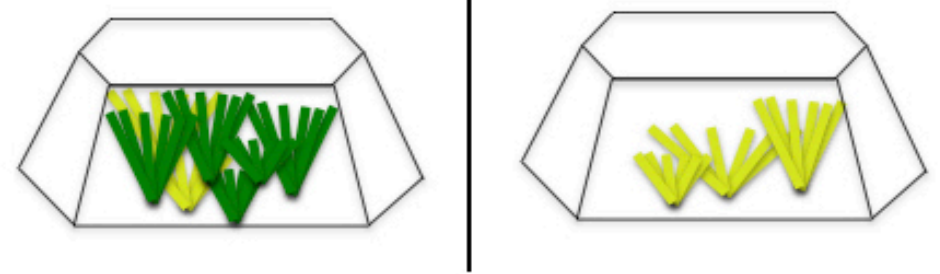

Figure 8. Diagram of the Warming and Dominant Species Removal (WaRM) experimental design. Green "plants" represent C. vulgaris. Warming plots demonstrate the application of Open-Top Chambers that passively warm the plots by $\sim 1.73^{\circ} \mathrm{C}$ higher than the controls. WaRM consists of 8 replicates plots of each treatment: ambient-control; ambient-removal; warming-control; warming-removal $(\mathrm{n}=32)$.

Additionally, research in the TNP WaRM project serves as a model ecosystem for the other 87 distinct alpine areas above 1000m within New Zealand's North Island. With a $3^{\circ} \mathrm{C}$ surface temperature rise, only 6 of the 87 areas are predicted to maintain abiotic conditions that will allow for the persistence of alpine plant communities, while additionally becoming significantly smaller in size. (Halloy and Mark, 2003) 
Plant species

C. vulgaris and 5 endemic plant species were selected for sampling. As the plant community consists of species that form either arbuscular (AM) or ericoid (ErM) mycorrhizas, I sampled the three species that represent the most common and abundant, $\mathrm{AM}$ and ErM plant species across all plots as measured by percent ground cover. Calluna vulgaris (CALVUL), Dracophyllum subulatum (DRASUB), and Epacris alpina (EPAALP) were selected as the representatives of the ericoid mycorrhizal plants. Chionochloa rubra (CHIRUB), Ozothamnus vauvilliersii (OZOVAU) and Veronica venustula (VERVEN) were selected as the representatives of the arbuscular mycorrhizal plants. In each plot, the largest individuals of each species were selected for sampling. Table 1 shows the mean percentage cover data of each host plant species in each plot by treatment. Surface cover data is regarded as a good indicator of below-ground biomass and therefore potential below-ground properties (Cheng and Niklas, 2007).

Table 1. Mean percentage cover of host plant species in the WaRM treatments. Number of individual host plants sampled per species sampled from the WaRM treatments; $\mathrm{n}=8$ plots per treatment. CALVUL, Calluna vulgaris CHIRUB, Chionochloa rubra. DRASUB, Dracophyllum subulatum. EPAALP, Epacris alpina. OZOVAU, Ozothamnus vauvilliersii. VERVEN, Veronica venustula.

\begin{tabular}{c|rrrrrr} 
TREATMENT & CALVUL & CHIRUB & DRASUB & EPAALP & OZOVAU & VERVEN \\
\hline $\begin{array}{c}\text { AMBIENT } \\
\text { CONTROL }\end{array}$ & 12 & 2.5 & 8 & 1.5 & 2.5 & 0.5 \\
AMBIENT & - & 3 & 8 & 2.5 & 1 & 0.5 \\
REMOVAL & 26 & 0.5 & 6 & 2 & 0.5 & 0.5 \\
WARM & & & & & & \\
CONTROL & 1.5 & 9 & 1.5 & 0.5 & 1 \\
WARM & - & & & &
\end{tabular}


Plant root tips were obtained by tracing lateral roots from the base of the selected individuals to the root tips. At least two terminal clusters $\sim 5 \mathrm{~cm}$ in length and containing of 10-20 root tips, were collected from each individual. This method of root sampling was tested by Chagnon and Bainard, (2014) and was determined as a reliable sampling method to represent mycorrhizal community structure. To limit damage to the experimental plots, only one individual per species, per plot, was sampled. However, it is important to note, most plots had one individual plant per species. Root tips were then stored in $1.5 \mathrm{~mL}$ Eppendorf tubes filled with RNALater and kept cool and out of direct sunlight until they were returned to the lab ( $\sim 48$ hours). All samples were then frozen to $-20^{\circ} \mathrm{C}$. Table 2 shows how the successful samples represent the treatments and species sampled.

Table 2. Number of individual host plants successfully sampled per species from the WaRM treatments; $\mathrm{n}=8$ plots per treatment. CALVUL, Calluna vulgaris CHIRUB, Chionochloa rubra. DRASUB, Dracophyllum subulatum. EPAALP, Epacris alpina. OZOVAU, Ozothamnus vauvilliersii. VERVEN, Veronica venustula.

\begin{tabular}{c|cccccc|c} 
TREATMENT & CALVUL & CHIRUB & DRASUB & EPAALP & OZOVAU & VERVEN & TOTAL \\
\hline $\begin{array}{c}\text { AMBIENT } \\
\text { CONTROL }\end{array}$ & 6 & 7 & 8 & 4 & 8 & 7 & $\mathbf{4 0}$ \\
$\begin{array}{c}\text { AMBIENT } \\
\text { REMOVAL }\end{array}$ & - & 7 & 7 & 7 & 7 & 5 & $\mathbf{3 3}$ \\
$\begin{array}{c}\text { WARM } \\
\text { CONTROL }\end{array}$ & 8 & 5 & 7 & 6 & 7 & 5 & $\mathbf{3 8}$ \\
$\begin{array}{c}\text { WARM } \\
\text { REMOVAL }\end{array}$ & - & 5 & 7 & 7 & 6 & 5 & $\mathbf{3 0}$ \\
\hline TOTAL & $\mathbf{1 4}$ & $\mathbf{2 4}$ & $\mathbf{2 9}$ & $\mathbf{2 4}$ & $\mathbf{2 8}$ & $\mathbf{2 2}$ & $\mathbf{1 4 1}$
\end{tabular}




\section{Molecular Methods}

The root tips were then washed of excess soil and RNALater in room temperature distilled water and separated into $300 \mathrm{mg}$ replicate samples, as per the recommendations of Chagnon and Bainard, (2014). Fungal DNA was then extracted from root tissues using a modified CTAB Extraction Method similar to that optimised by Thakuria et al., (2008). I modified this protocol in the following manner; root tip tissue was homogenised in a $1.5 \mathrm{~mL}$ tube with RNase free $0.7 \mathrm{~mm}$ steel lysis beads with $500 \mu \mathrm{L}$ of CTAB Extraction buffer at $(2 \%$ cetyl trimethylammonium bromide, $1 \%$ polyvinyl pyrrolidone, $100 \mathrm{mM}$ Tris-HCl, 1.4 M NaCl, $20 \mathrm{mM}$ EDTA) at 2 minutes of 7000rpm in Precellys ${ }^{\mathrm{TM}}$ Evolution tissue homogeniser. This method is considered harsh for DNA extractions (de Boer et al., 2010). However, five of the six species sampled had woody roots that did not yield sufficient DNA using gentler methods. Lysis bead diameter and centrifuge speeds were tested and optimised for the tradeoff between DNA yield and shearing.

The DNA pellet was then eluted in $20 \mu \mathrm{L}$ room temperature TE buffer $(10 \mathrm{mM}$ Tris, $\mathrm{pH} 8,1 \mathrm{mM}$ EDTA). With pellets that were stubborn in dissolving, the samples where incubated for 2 minutes in $30^{\circ} \mathrm{C}$. The DNA precipitate was then eluted with ultra distilled water, resulting in a final product of $20 \mu \mathrm{L}$ of DNA product per sample. The final DNA product was then tested for its concentration on a Nanodrop ${ }^{\mathrm{TM}}$ spectrophotometer to test if it had a concentration of $>10 \mathrm{ng} / \mu \mathrm{L}$. Of the 155 samples collected from the field, all

passed this quality test, with a few samples requiring re-extraction from replicate material. These DNA samples were kept frozen at $-80^{\circ} \mathrm{C}$ until utilised in the Polymerase Chain Reaction (PCR) amplification method. 
For PCR, 2 variants of ITS3 primers and 20 variants of ITS4 were selected to create 40 unique forward and reverse bar-coded PCR products. The primers selected were designed to target the ITS2 region (250-300 base pairs) which is recognised as the optimal region for parsing fungal diversity from sequence data (Blaalid et al., 2013; Bazzicalupo et al., 2013). The ITS primer sequences I used are listed in Appendix 1. Table 1. I followed the Touchdown PCR protocol as outlined by Korbie and Mattick, (2008). This methodology lowers the annealing temperature by approximately $0.5^{\circ} \mathrm{C}$ for each thermocycle, as primer annealment at higher temperatures are the least-permissive of nonspecific binding. This means the first sequence amplified is between the regions of greatest primer specificity. Therefore these sequences will continue to be exponentially amplified at to higher concentrations during subsequent rounds at lower temperatures. The result of this priority-style amplification is that sites with greater specificity will outcompete the nonspecific sequences, resulting in "cleaner" amplifications. The thermocycling protocol was modified from the Korbie and Mattick ,(2008) method, with the following conditions; $3 \mathrm{~min}$ initial denaturation at $99^{\circ} \mathrm{C}, 35$ cycles of $45 \mathrm{~s}$ denaturation at $95^{\circ} \mathrm{C}, 45 \mathrm{~s}$ annealing at $50^{\circ} \mathrm{C}$ (decreasing by $0.5^{\circ} \mathrm{C}$ cumulatively each cycle); and $1 \mathrm{~min}$ elongation at $72^{\circ} \mathrm{C}$; and a $15 \mathrm{~min}$ final elongation; resting at $4{ }^{\circ} \mathrm{C}$. All PCR amplification reactions contained 10ng of root DNA, $500 \mathrm{nM}$ of both primers, 1U RedTaq DNApolymerase (Sigma Aldrich, NZ), 1x TBT-PAR, 500mM dNTPs mix (all reagents from Sigma Aldrich, NZ).

The quality and quantity of the 250-300bp PCR products were then tested by gel electrophoresis, using a $1 \mathrm{~kb}$ DNA quantification ladder and the UVtech ${ }^{\mathrm{TM}}$ programme. 
Samples that failed to amplify, or that produced non-specific products were extracted and amplified again from replicate material. PCR amplicons were obtained for 152/155 of the root samples. Four pools of 40 unique forward and reverse bar-coded PCR products were then transferred in equal molar quantities into separate Eppendorf tubes, each pool representing a single multiplexed library for sequencing. The 4 pooled samples were cleaned of non-specific product by gel electrophoresis and extraction using a New England Biolabs gel extraction kit. The cleaned samples were then dehydrated in an Eppendorf rotary evaporator prior to shipping.

\section{Sequencing and Bioinformatics}

The dehydrated pooled samples were then sequenced on the Illumina Mi-Seq platform with Macrogen, South Korea. Each multiplexed library sample was rehydrated to $10 \mu \mathrm{L}$ with UltraPure double distilled water. Prior to sequencing, samples were confirmed with Quality Assurance tests to have peaks showing the targeted base pair length of the ITS2 region. The samples were then ligated with Illumina adapters and underwent high-throughput sequencing on the Illumina Mi-Seq platform. Upon completion, I received 2 FastQ files for each pooled library sample, representing the complimentary paired-end reads.

As Illumina adapters were added to the prepared amplicons only prior to sequencing, the adapter ligated indiscriminately to both the reverse and forward primers 3' ends. Therefore, the sequencing data files were a mix of paired-end reads, with combinatorial dual indexes, containing forward and reverse reads intermingled. Due to 
the nature of this sequencing data, the demultiplexing procedure was required to address the two distinct components of this data separately, 1) The combinatorial indexes (multiple combinations of forward and reverse primers in one output file), 2) The mixed Paired-End reads in files (forwards and backward primers not read in their assigned direction due to the indiscriminate ligation of the adapters). Both of these steps were completed using the Cutadapt program (v.2.10) (Martin, 2011). It is also important to note in the following steps described, the output sequence data files received from Macrogen already had the Illumina adapter sequences removed.

For the identification of the combinatorial indexes, (the primer barcodes), I used the cutadapt function from the Cutadapt program in conjunction with the 2 sample library FastQ files, 2 lists primer barcodes, labelled forward and reverse (Appendix 1. Table 1) and the list of specific primer combinations to be assigned a unique sample name. Primers were assigned as anchored, with an overlap of $5 \mathrm{bp}$ (my primers were 6bp long) with an error read of 0 . These steps ensured no primers were mis-identified for one another. The execution of this function found primer combinations within the sequence read data, removed them, and re-wrote the amplicon sequence data in the forward orientation into sample-separated files. Next, the previous function was run against the 4 data libraries again, however for this step, the primer sequences were reversed in their orientation. This accounted for the mixed paired-end reads, where the ligation of the Illumina adapter occurred on the reverse primer rather than the forward. The cutadapt function re-wrote the amplicon sequence data in the forward orientation to ensure correct taxonomic identification. 
Following this step, the complimentary files of the same sample identities from the previous two stages were merged together using the cat function in Terminal on the Macintosh OS. All of the re-written amplicon reads were correctly identified and matched with its corresponding sample name. A total of 8,003,522 read pairs were processed, of which 7,823,377 passed filters and were re-written into the corresponding 152 sample files. Of the 152 samples, 5 samples returned with $<4000$ sequence reads, I elected to discard these samples as the other 147 were evenly weighted with $\sim 977,000$ reads per sample.

For the taxonomic identification of the sample, I employed the automated fungal ITS pipeline, PIPITS devised by Gweon et al., (2015). PIPITS is an open-source standalone suite for processing of Illumina Mi-Seq sequences for fungal community analysis. It is the first automated bioinformatics pipeline designed for fungal ITS sequences from the Illumina Mi-Seq platform. The pipeline also utilises the latest version of RDP Classifier, (Wang et al., 2007) to classify sequences against the UNITE fungal data set. RDP Classifier was identified as the most accurate in discerning the most similar sequences (Cole et al., 2014), as well as applying a confidence ranking for each of level of taxonomic assignment (Wang et al., 2007). It is currently recognised as the most powerful tool for the high-throughput identification of fungal ITS sequences (Nilsson et al., 2019).

The pipeline used for my method had three key steps, the first, PIPITS_PREP which prepared the raw demultiplexed sequence reads from the Cutadapt output. This step retrieved a list of all read-pairs from the raw sequence directory. Then it identified 
and joined all overlapping regions of sequences with PEAR (Zhang et al., 2014b) and used the read-pairs list to reindex all sequences to be read in the same direction. Finally it applied a quality filtering step, which removed sequences with unknown nucleotides $(\mathrm{N})$, and converted these filtered reads in single FastA file format.

The second step, PIPITS_FUNITS, initially removed the redundant replicate sequences and short sequences of $<100 \mathrm{bp}$. Then ITS2 subregions of fungal origin were then extracted from the sequences. Where required, sequences were re-orientated to 5'-3' direction. These filtered ITS2 fungal sequences were then re-inflated to represent their original abundance to a total of 2,238,212.

The final step of the pipeline, PIPITS_PROCESS, used VSEARCH (Rognes et al., 2016) for clustering sequences into Operational Taxonomic Units (OTUs). The pipeline removed unique sequences prior to clustering them to a threshold of $97 \%$ sequence identity. The output of representative sequences for each cluster were then subjected to chimera detection and removal using the UNITE UCHIME reference data bases. After this removal, the resulting 2,170,651 ITS2 sequences were then taxonomically assigned with the RDP Classifier against the UNITE fungal ITS reference data base. This assignment identified individual 3067 OTUs and 466 phylotypes. Two output tables were produced, an 'OTU abundance table' (OTUs represent a species level threshold) and 'phylotype abundance table' (where an OTU is defined as a cluster of sequences grouped into a higher level of taxonomic assignment). From here, 6 of the 147 remaining samples were discarded due to producing read numbers $<1000$, as the median read of samples was 45,251 . 
To identify the ecological guild and functional role of these assigned OTUs, the OTU abundance table from the PIPITS_PROCESS output was applied to FUNGuild analysis (Nguyen et al., 2016). FUNGuild is a Python-based tool used to taxonomically resolve fungal OTUs by ecological guild. This analysis automated the assignment of ecological roles to the fungal taxa, including the identification of mycorrhizal species. The output was a table of total sequence reads in each sample for the OTUs that were assigned ecological roles, with the ecological roles ranked by likelihood. Of these 3067 OTUs, 53 were assigned as "highly likely" belonging to an arbuscular mycorrhizal guild and 47 were assigned "highly likely" as ericoid mycorrhizal guild. This table formed the basis of all of the following statistical methods. 


\section{Statistical Methods}

To ensure that the fungal OTUs collected across the sampled plant species accurately represented the total expected fungal OTU community, I conducted a collector's curve analysis using the vegan package using $\mathrm{R}$ studio software (v. 3.6.3). I used the collectors.curve function, which plotted the cumulative number of fungal OTUs (3067) as a function of the number of plant root samples. In addition, I then plotted fungal OTU accumulation by WaRM treatment as a function of the number of sequence reads (i.e., a rarefaction curve) using the rarecurve function in the vegan package in $\mathrm{R}$. This step allowed me to assess whether sampling was equally and adequately applied across the experimental treatments. Similarly, to assess whether sampling was equally and adequately applied across the plant species, I also plotted fungal OTU accumulation by plant species as a function of the number of sequence reads.

To visualise the overall functional composition of the fungal community, I calculated the proportional abundances of the detected fungal ecological guilds (i.e., saprotroph, arbuscular mycorrhiza, endophytes ect.) per treatment and per plant species using pivot charts. I considered two measures of proportional abundance; total sequence reads per ecological guild, and OTU richness per ecological guild. I then created comparative stacked column graphs of this data in Microsoft Excel (v.16.40). I used chisquare tests using the CHISQ.TEST function in Excel to identify significant differences among treatments and plant species in the proportional abundances of fungal guilds.

To test if I had adequately sampled the mycorrhizal fungal composition across the WarM treatment and six plant species, I applied the aforementioned analysis again, using 
the rare.curve function in $\mathrm{R}$, plotting the mycorrhizal fungal (AM+ErM) OTU accumulation by WaRM treatment and by plant species as a function of the number of sequence reads. I standardised the total sequences reads for each sample to the overall mean read count per sample. I then created stacked bar charts of the standardised read count of both AM and ErM OTUs by treatment and by species. I used chi-squared tests to identify significant differences among treatments and plant species. This analysis revealed low numbers of sequence reads of ErM fungal OTUs on AM host plant species and vice versa. Therefore, I focused subsequent diversity analyses on the mycorrhizal functional guild taxa across all six plant species.

I calculated Shannon Diversity, Species Richness, and Pielou's Evenness indices for the standardised OTU reads using the vegan package in R. I selected these as indices as comprehensive and complimentary measures of diversity. Shannon diversity $\left(\mathrm{H}^{\prime}\right)$ is a diversity metric that also accounts for species' evenness between samples (Shannon, 1948), using the formula as follows:

$$
\mathrm{H}^{\prime}=\sum_{\mathrm{i}=1}^{s} p_{\mathrm{i}} \ln p_{\mathrm{i}}
$$

Where H' is Shannon's index value, $p_{\mathrm{i}}$ is the proportion of OTUs found in the $i$ th species, $\ln$ is the natural logarithm and $s$ is the total number of species. Species richness is the count of OTUs. In addition, Pielou's evenness, a measure of taxonomic distinctness between samples, was calculated by dividing the calculated Shannon Diversity value by $\log$ (richness) (Pielou, 1966; Smith and Wilson, 1996). I assessed these three diversity 
indices as a function of the WaRM treatments for each host plant species. The data outputs were then tested for normal distribution using a Levene's test using the levenes test function in the car package in R. As the assumptions were met for all diversity indices, a two-way analysis of variance (ANOVA) was then conducted, using the aov function in R, for each species and each diversity analyses tested. Post-Hoc Tukey HSD tests were used to determine pairwise differences among treatments using the tukeyhsd function in R. Box plots were used to illustrate the responses of diversity indices to treatments.

\section{Multivariate analyses}

To determine the total dimensionality of the mycorrhizal fungal sequence read data, I used non-metric multidimensional scaling (NMS) as implemented in PC-ORD software (version 7.08). The primary advantage of using NMS over other ordination methods (e.g., Canonical Correspondence Analysis), is that the total variance within the data determines the dimensionality and stress of the solution. I applied NMS Ordinations to the matrix of mycorrhizal OTU sequence read data, by the 141 plant roots sampled. Cell values of the matrix were weighted by standardised sequence read count per root sample, and square-root transformed. Although NMS does not exclusively rely on a normal distribution or the assumption of linear relationships among variables, McCune and Grace, (2002) recommend this data transformation for sequence read data, given the large number of zero values in these matrices. 
NMS Ordinations were then run using the PC-ORD software in the 'auto-pilot' mode, which performed 500 iterations, consisting of 250 runs of both the real data and randomly generated data. Each iteration of ordination started with random configurations of data, and assessed its dimensionality (number of axes determining a solution) by minimising stress. Dissimilarity between samples was measured by Euclidean (Pythagorean) distance. Ordination solutions were then plotted within a two-dimensional space on the two axes that explained the largest proportion of variance between the samples. I replicated the plots of the ordination solution, so samples were categorised by their WaRM treatment, host plant species and their plot number. This was to visualise if these experimental variables determined a significant proportion of the total variance within the data.

I then tested for significant pairwise differences in the mycorrhizal fungi community structure of the variables. For this analysis, I applied the data matrix to a multiple response permutation procedure (MRPP) as implemented in PC-ORD software. While my factorial experimental design naturally lends itself to testing the significant effects of these variables through MANOVA, my data does not meet its assumptions of equal numbers of samples per variable group. Nonetheless, MRPP is recognised as more powerful than MANOVA with multivariate normal data, as non-linear relationships and asymmetric distributions can skew the significance of effects detected by MANOVA (Smith, 1998). Although MRPP does not require normality, I used the same transformed data matrix of the NMS analysis, to ensure for the most statistical power, and consistency of approach between NMS and MRPP. MRPP then calculated a chance-corrected within- 
group agreement (A) using Euclidean distance measures for both WaRM treatment effect, species effect and plot effect. The chance-corrected within-group agreement (A), is a measure of within-group homogeneity compared to that expected by chance. Where $\mathrm{A}=$ 1 , this equates to identical members within each given group, and $\mathrm{A}=0$ corresponds to within-group heterogeneity equal to or larger than that expected by chance. The MRPP results were then recorded in a table with corresponding NMS plots.

I used NMS and MRPP analyses in an investigative approach to identify which variables were determining the largest degree of variance in MF community structure. For the investigation I initially tested all samples being plotted in the ordination space of all mycorrhizal OTUs (100). I then split the data matrix by the arbuscular mycorrhizal (AM) and ericoid mycorrhizal (ErM) guilds; with all AM host plant samples plotted within AM fungi ordination space, and all ErM host plant samples plotted within ErM fungi ordination space. Finally, I independently plotted samples of each host-plant species in their associated MF ordination space. This was to determine if treatment effects were significant on the mycorrhizal communities at a host-plant species level. All multivariate analyses of these data subsets were as described above. 
Results

\section{Total Fungal Community}

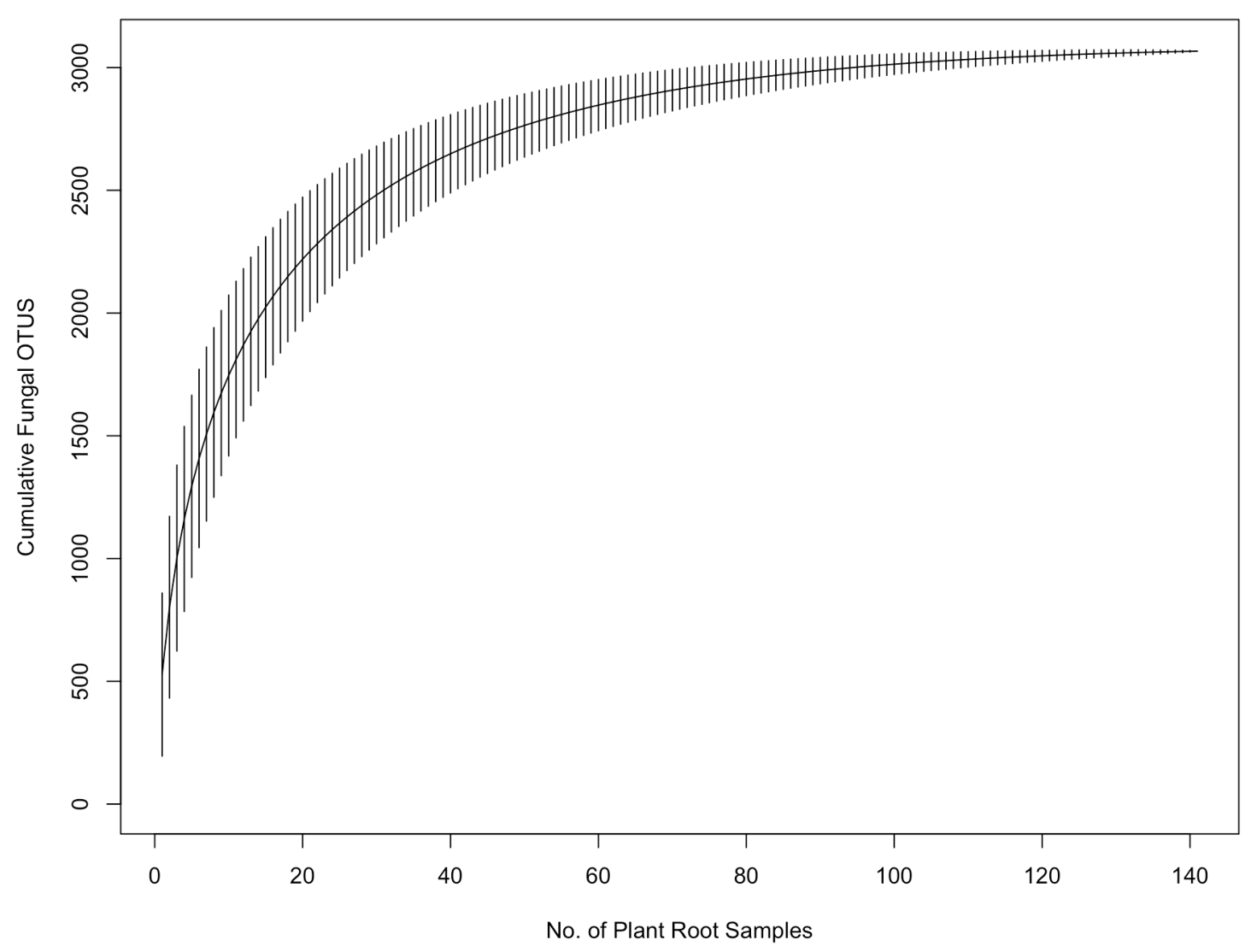

Figure 9. The cumulative number of fungal OTUs detected as a function of the number of plant root samples (i.e., a Collector's accumulation curve), using the collectors.curve from the plot.rtk package in R. The flattening of the curve and narrowing of standard error suggests that I have sufficiently sampled the fungal community associated with the roots of 6 alpine plant species.

The collector's accumulation curve analysis (Figure 9) revealed that total fungal OTU richness (3067 OTUs), as expected from multiple iterations of OTU turnover between random samples, was captured within the 141 samples. This confirms I applied the appropriate sampling effort to capture the total fungal diversity expected within my 141 samples. 


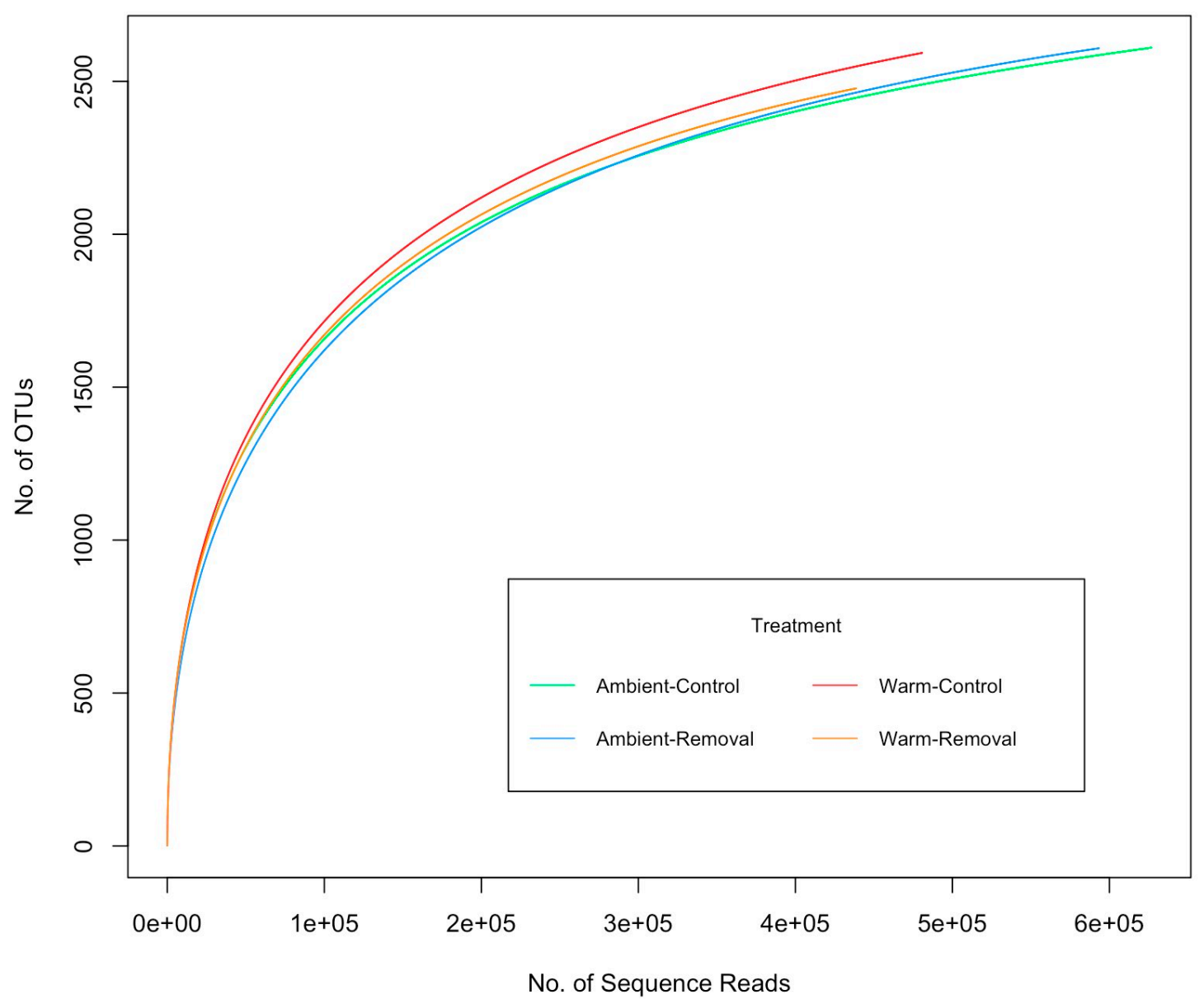

Figure 10. Fungal OTU accumulation by WaRM treatments as a function of the number of sequence reads (i.e., rarefaction curve), from rare.curve function from the vegan package in R.

Rarefaction of fungal OTUs by treatment (Figure 10) revealed that total fungal OTU richness, as expected from total sequence reads, was not sufficiently captured with any treatment, as ongoing OTU turnover, prevented the "flattening" of the curves. This confirms I have not exhaustively sampled all fungal diversity in each treatment. WaRM treatments did not exhibit a significant difference in the richness of total fungal OTUs relative to the ambient control treatment, however ambient plots have a higher number of sequences reads than warm plots by a factor of $1 \mathrm{E} 05$. 


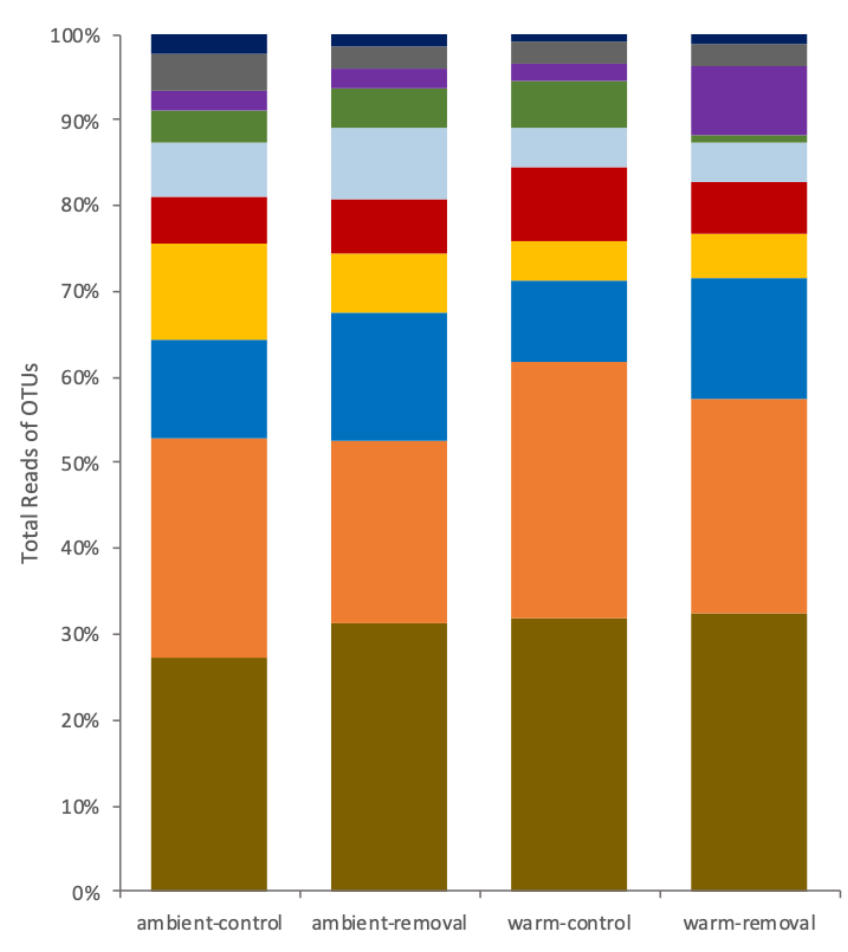

(A)

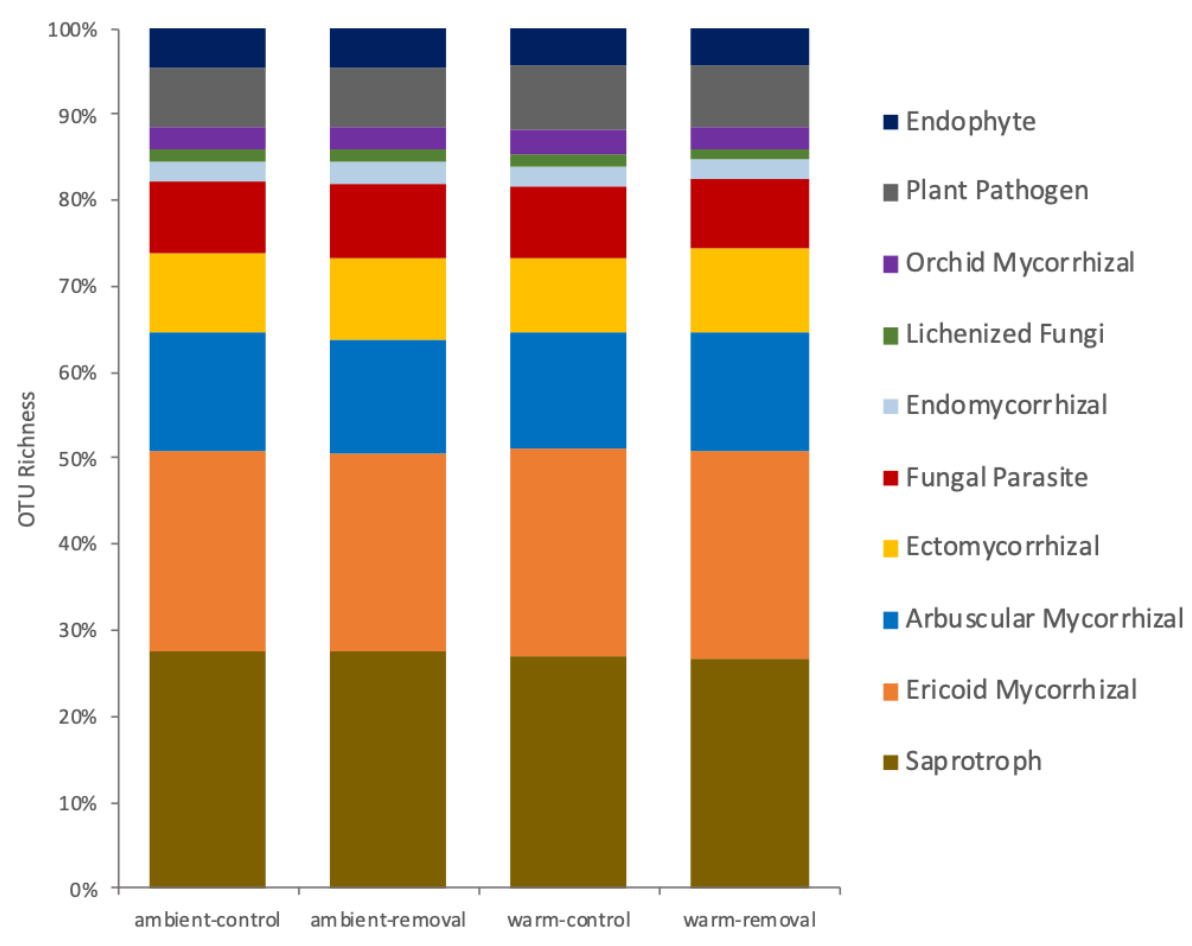

(B)

Figure 11. The proportional abundance of fungal functional guilds in the WaRM treatments as determined by (A) read count and (B) OTU richness. OTU richness was calculated using the vegan package in $\mathrm{R}$.

The analysis of proportional abundance of fungal functional guilds as a response to the WaRM treatments (Figure 11A) reveals an increase in ericoid mycorrhizal fungi of sequence reads in the warm-control treatment. However, chi-square tests found no significant differences proportional abundance of OTU sequence reads or richness between WaRM treatments for any functional guild. 


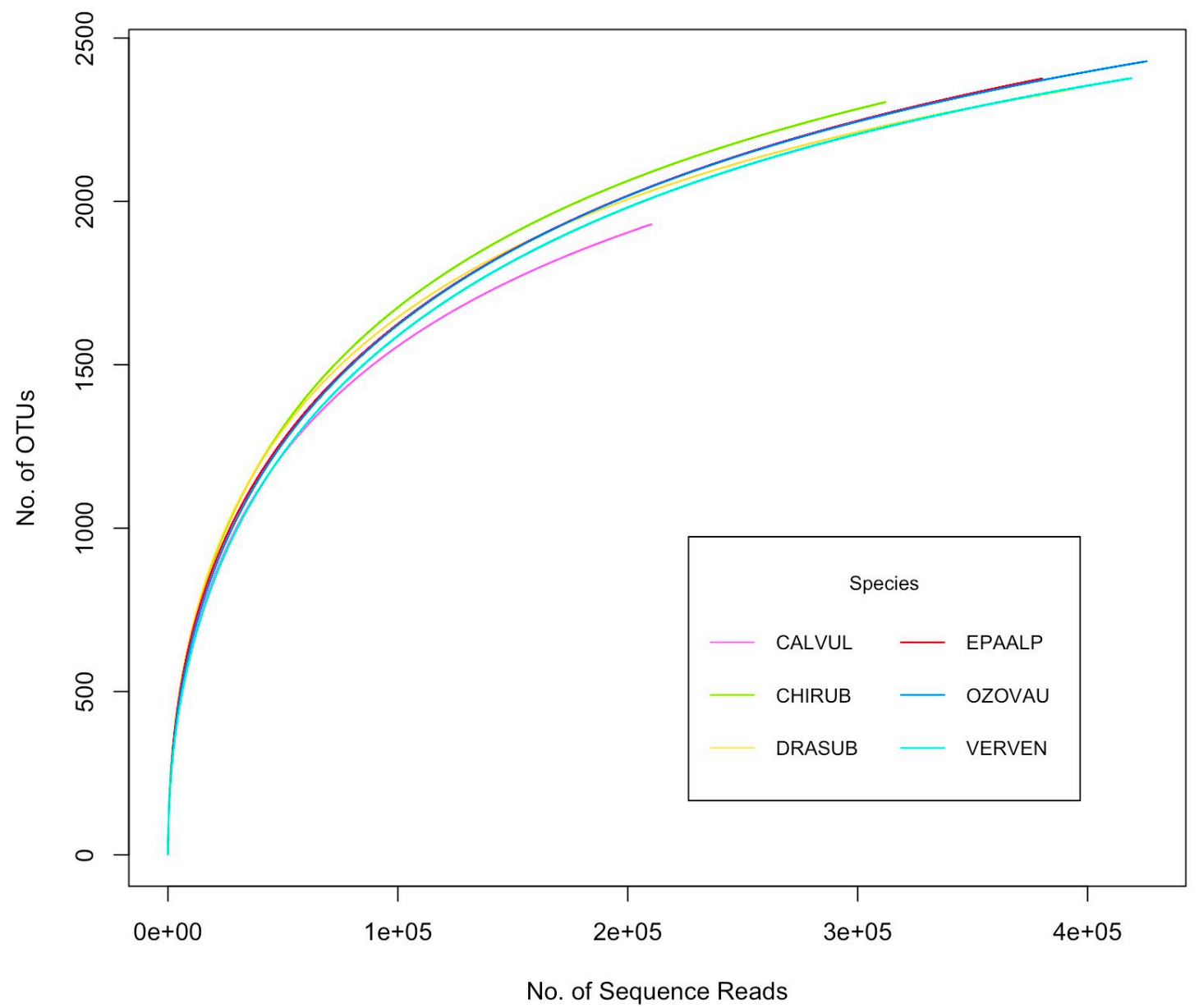

Figure 12. Fungal OTU accumulation by host plant species as a function of the number of sequence reads (i.e., rarefaction curve) from the rare.curve function from the vegan package in R. Note that Calluna vulgaris (CALVUL) is removed from half of the plots. CALVUL, Calluna vulgaris CHIRUB, Chionochloa rubra. DRASUB, Dracophyllum subulatum. EPAALP, Epacris alpina. OZOVAU, Ozothamnus vauvilliersii. VERVEN, Veronica venustula.

Rarefaction of fungal OTUs by species (Figure 12) revealed that total fungal OTU richness, as expected from total sequence reads, was not sufficiently captured within any species, as ongoing OTU turnover, prevented the "flattening" of the curves. This confirms I have not exhaustively sampled all fungal diversity in each species. Calluna vulgaris had less sequence reads than other host plants by a factor of $2 \mathrm{E} 05$, as result of its removal from half of the plots in the WaRM treatment. 


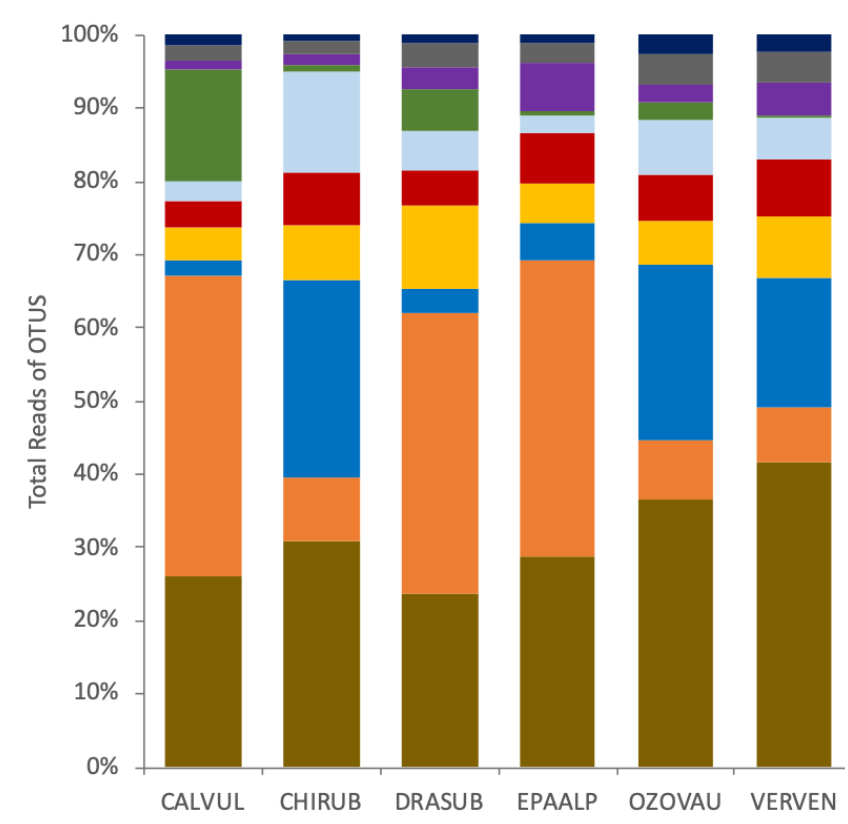

(A)

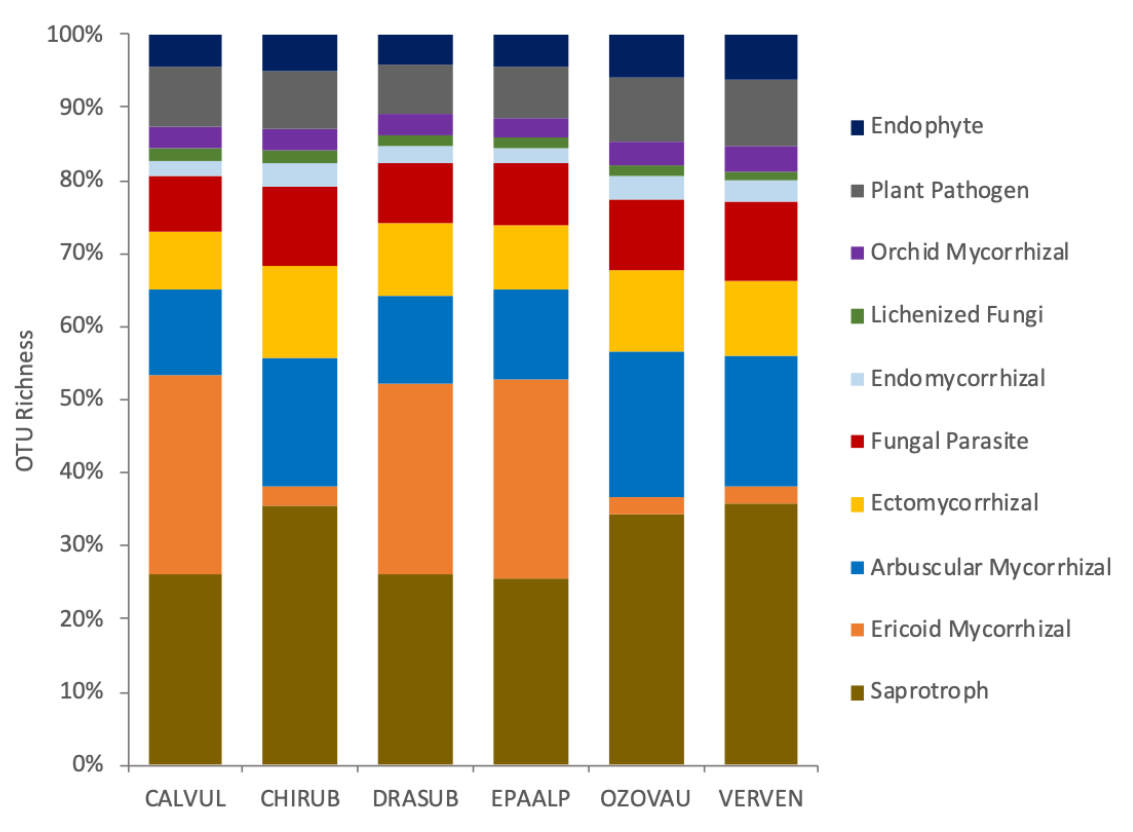

(B)

Figure 13. The proportional abundance of fungal functional guilds in the host plant species as determined by (A) read count and (B) OTU richness. OTU richness was calculated using the vegan package in R. CALVUL, Calluna vulgaris CHIRUB, Chionochloa rubra. DRASUB, Dracophyllum subulatum. EPAALP, Epacris alpina. OZOVAU, Ozothamnus vauvilliersii. VERVEN, Veronica venustula.

The analysis of proportional abundance of fungal functional guilds within host plant species (Figure 13A) reveals a strong pattern occurring in the arbuscular (AM) and ericoid (ErM) mycorrhiza reads across species. ErM fungi were significantly more abundant (chi-square $=21.18, \mathrm{p}<0.0001$ ) and had significantly higher taxonomic richness (chi-square $=7.92 \mathrm{p}=0.0038)$ on ErM host plant species $(C$. vulgaris, $D$. subulatum, E. alpina) than on AM host plant species (C. rubra, O. vauvilliersii, $V$. venustula). Chi-square tests found no significant differences in the ErM abundance or ErM OTU richness on AM host species. 
Conversely, AMF were significantly more abundant $($ chi-square $=14.29, \mathrm{p}$ $<0.0002)$ on AM host plant species than ErM host plant species. However, chi-square tests found no significant difference in AMF OTU taxonomic richness between AM host plant species and ErM host plant species, or between any individual host plant species. This suggests that while AMF are much more abundant among AM hosts, ErM host plants are indiscriminate towards AMF taxa. This also suggests that the ITS primers I employed are sensitive to the Glomeromycota regardless of their abundance. In addition, there were no significant differences in the AMF abundance or AMF OTU richness between ErM host species. 
Mycorrhizal Fungal Community_

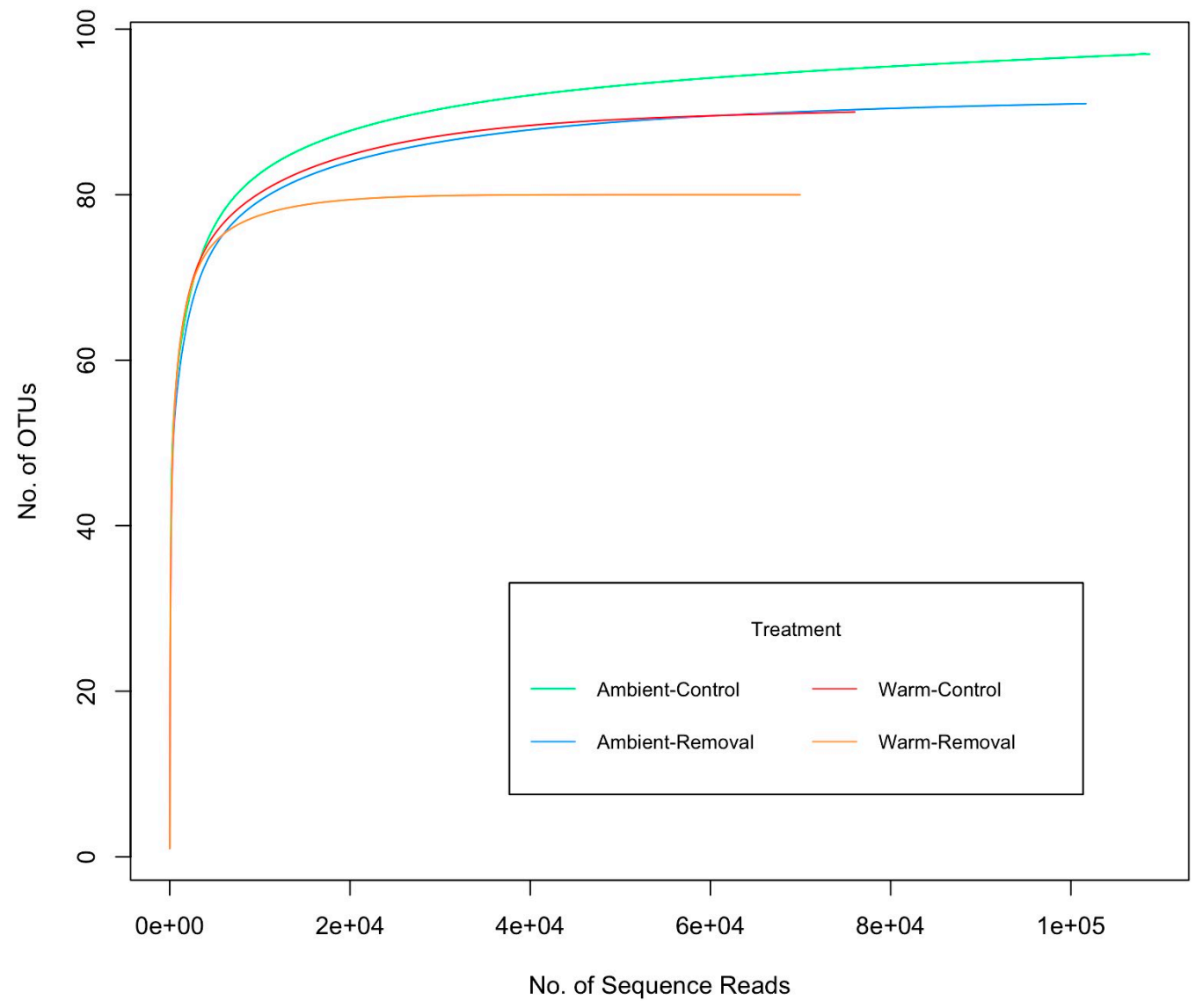

Figure 14. Mycorrhizal fungal (MF) OTU accumulation by WaRM treatments as a function of the number of sequence reads (i.e., rarefaction curve), from the rare.curve function from the vegan package in $\mathrm{R}$.

Rarefaction of mycorrhizal fungal (MF) OTUs by treatment (Figure 14) revealed that MF OTU richness, as expected from total sequence reads, was sufficiently captured within each treatment. This confirms I have applied sufficient sampling effort to all mycorrhizal fungal diversity expected in each treatment. In addition, the analysis shows that WaRM treatments reduced the richness of mycorrhizal OTUs relative to the ambient control treatment. Moreover, warming and dominant species removal treatment appear to act synergistically to cause the greatest reduction in the richness of mycorrhizal taxa. 


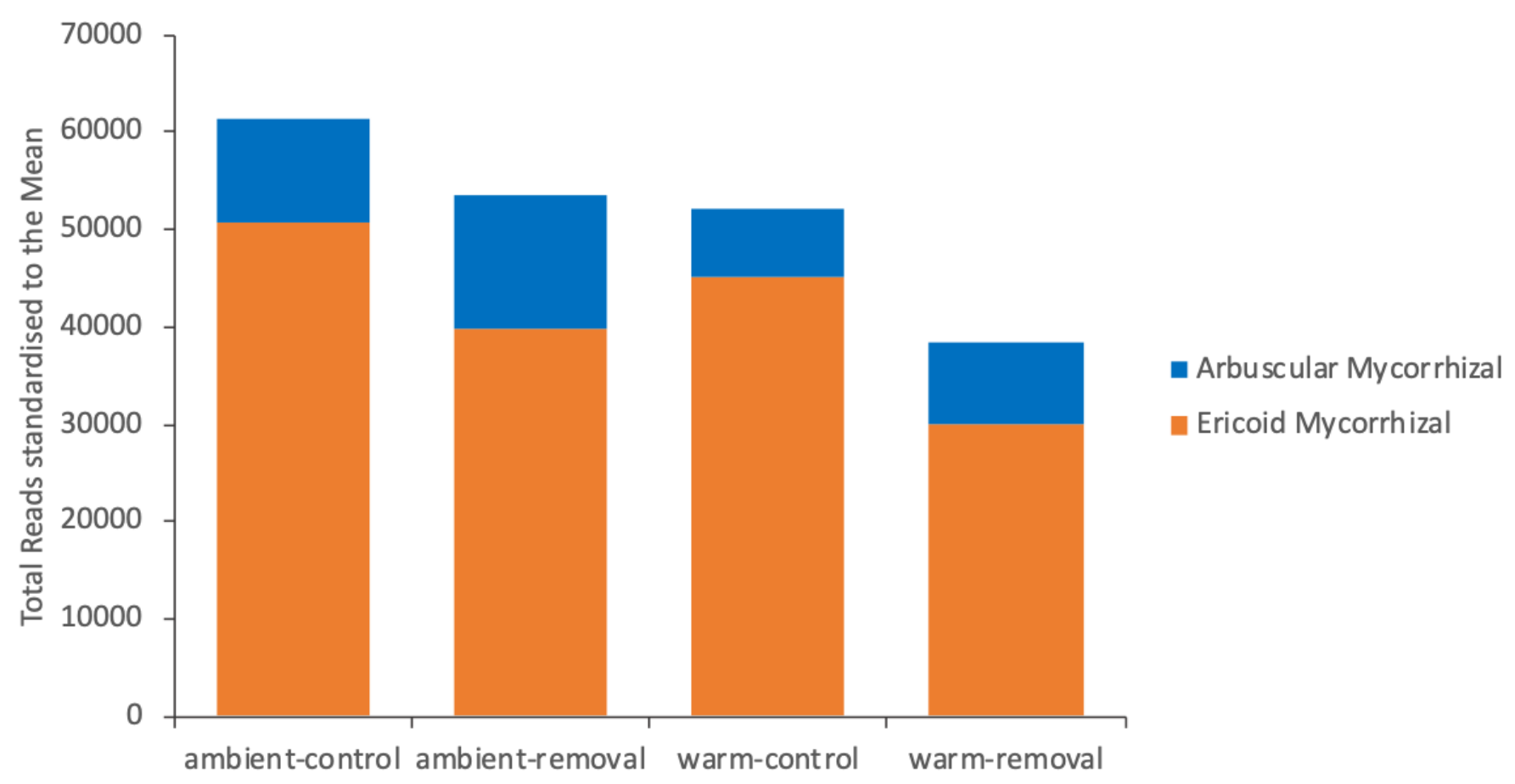

Figure 15. Standardised sequence reads of AMF and ErM fungi by WaRM treatment. Read counts are standardised to the mean of the total number of reads by species.

The analysis of standardised reads of mycorrhizal fungal guilds by WaRM treatment (Figure 15) reveals that warming treatment reduced AMF OTU abundance and increased ErM OTU abundance. However, chi-square tests only found a significant decrease in AM OTUs in the warm-control treatment when compared to ambientremoval. (chi-square $=5.42, \mathrm{p}=0.02$ ). There was no significant difference detected in chi-square tests in MF OTU abundance between any other treatments. 


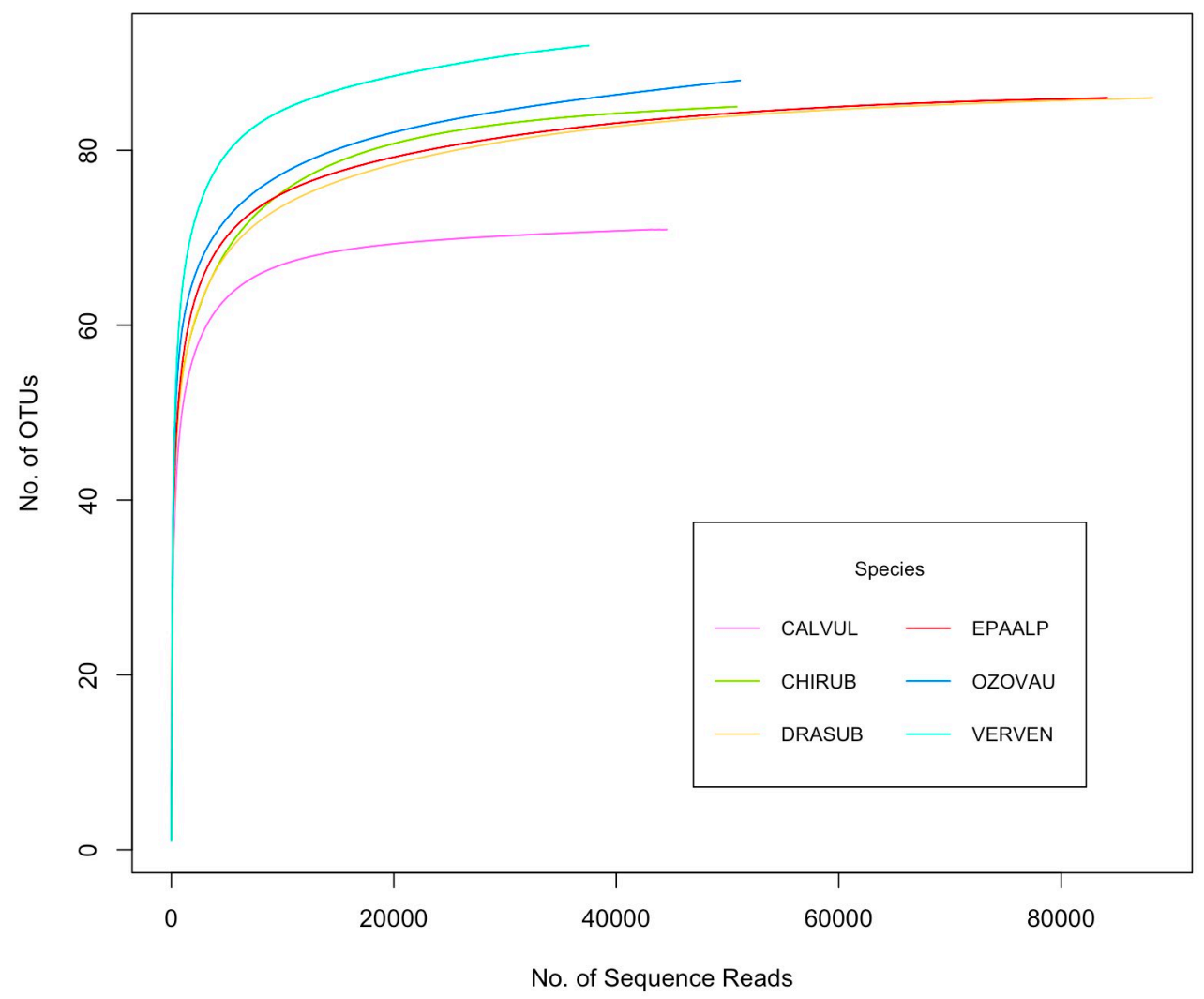

Figure 16. Mycorrhizal fungal (MF) OTU accumulation by host plant species as a function of the number of sequence reads (i.e., rarefaction curve) from the rare.curve function from the vegan package in R. Note that CALVUL is removed from half of the plots. CALVUL, Calluna vulgaris CHIRUB, Chionochloa rubra. DRASUB, Dracophyllum subulatum. EPAALP, Epacris alpina. OZOVAU, Ozothamnus vauvilliersii. VERVEN, Veronica venustula.

Rarefaction of mycorrhizal fungal (MF) OTUs by species (Figure 16) revealed that MF OTU richness, as expected from total sequence reads, was sufficiently captured within each species. This confirms I have applied sufficient sampling effort to all MF diversity expected in each species. Calluna vulgaris had less sequence reads compared to other host plants, as result of its removal from half of the plots in the WaRM treatment, however it was also found to have a lower number of MF OTUs. This raises a question as to whether $C$. vulgaris is less generalist to MF than the other host plants, or whether $C$. vulgaris exhibits a higher degree of selectivity against non interacting MF OTUs. 


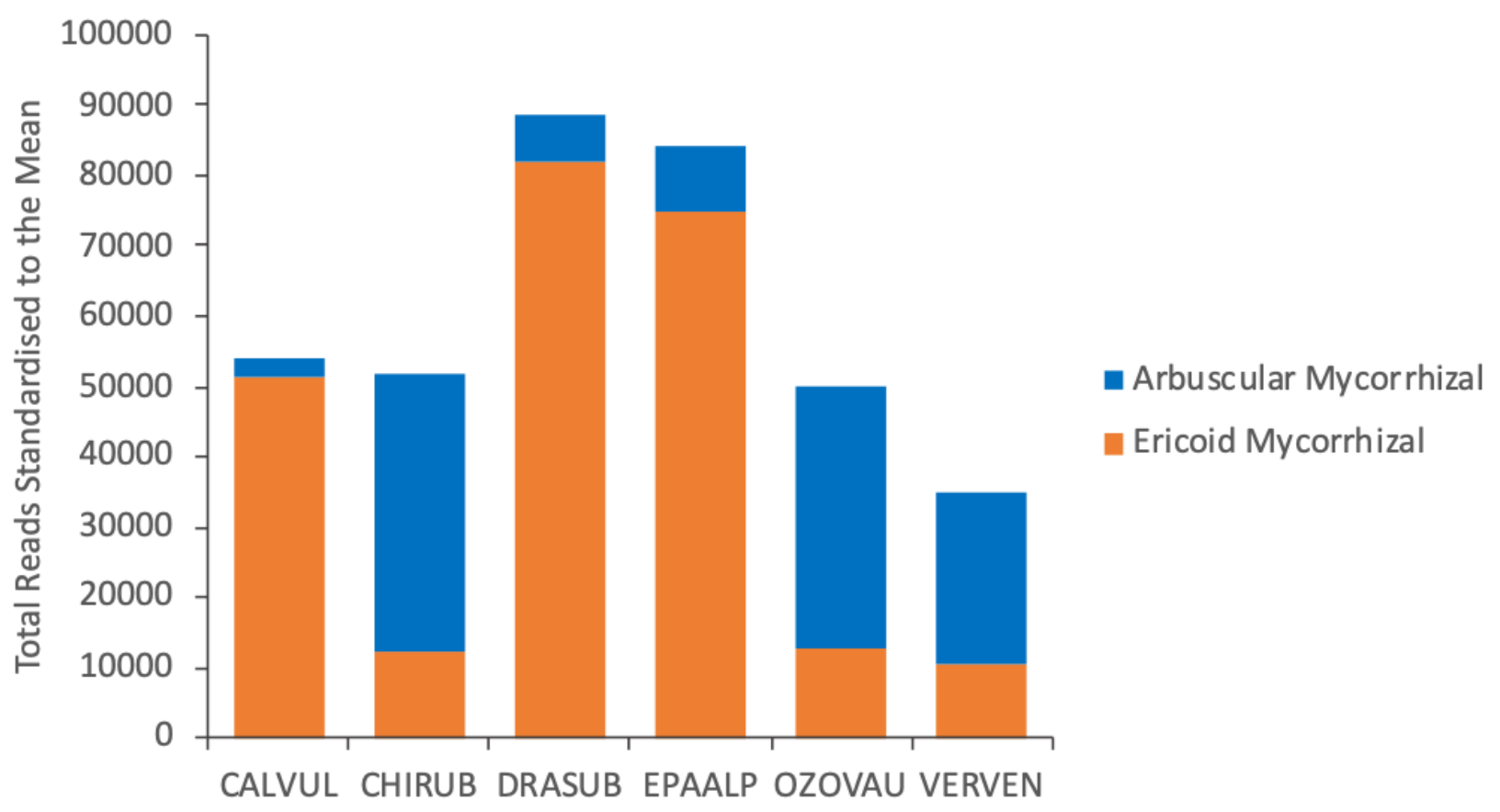

Figure 17. Standardised sequence reads of AMF and ErM fungi by species. Read counts are standardised to the mean of the total number of reads by species. CALVUL, Calluna vulgaris. CHIRUB, Chionochloa rubra. DRASUB, Dracophyllum subulatum. EPAALP, Epacris alpina. OZOVAU, Ozothamnus vauvilliersii. VERVEN, Veronica venustula.

The analysis of standardised reads of MF guilds by host plants species (Figure 17), confirms a significant difference in MF guild OTU abundance between AM hosts and ErM hosts. AMF abundance was significantly higher in AM hosts (chi-square $=$ 26.53, $\mathrm{p}<0.0001$ ). In turn, ErM abundance was significantly higher in ErM hosts (chisquare $=26.93, \mathrm{p}<0.0001)$. Chi-square tests found no significant differences in AMF OTU abundances between AM hosts (chi-square $=2.82, \mathrm{p}=0.09$ ) or in ErM OTU abundances between ErM hosts (chi-square $=2.91, \mathrm{p}=0.08$ ) despite the figure suggesting a difference in ErM OTU abundance between C. vulgaris and the native ErM hosts. Even though $C$. vulgaris was removed from half of the experimental plots, it did not possesses significantly lower abundances of ErM or AM OTUs overall to ErM hosts. Moreover, these results do not confirm nor negate the generalist status of C. vulgaris in comparison to native host plants. 
Treatment effect on diversity at a species level

Shannon Diversity
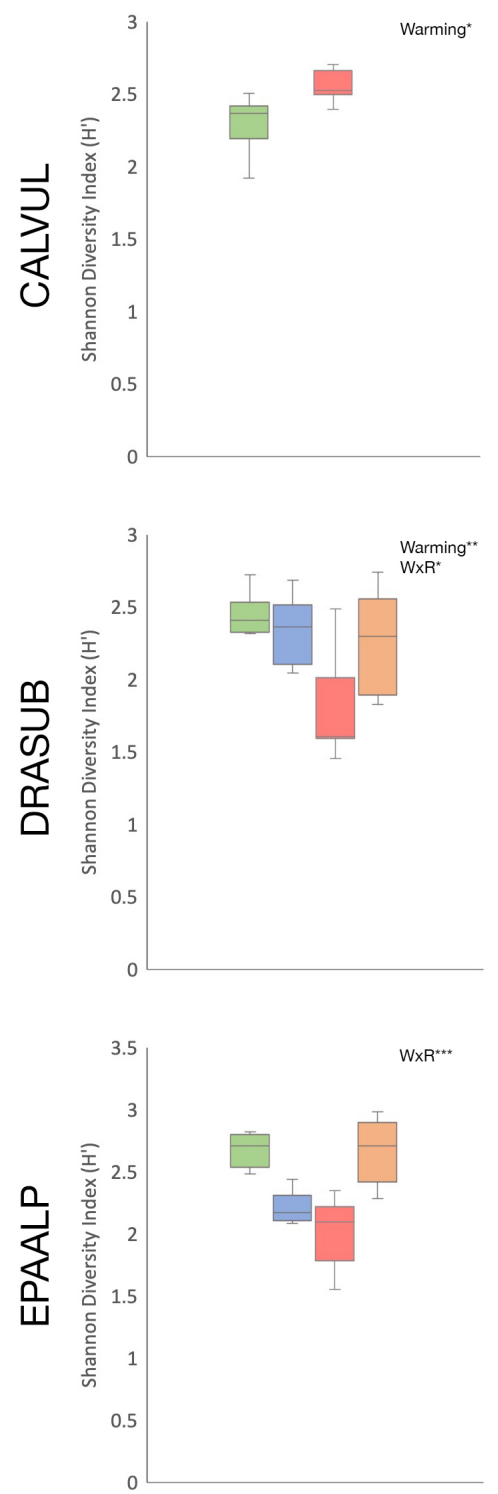

OTU Richness
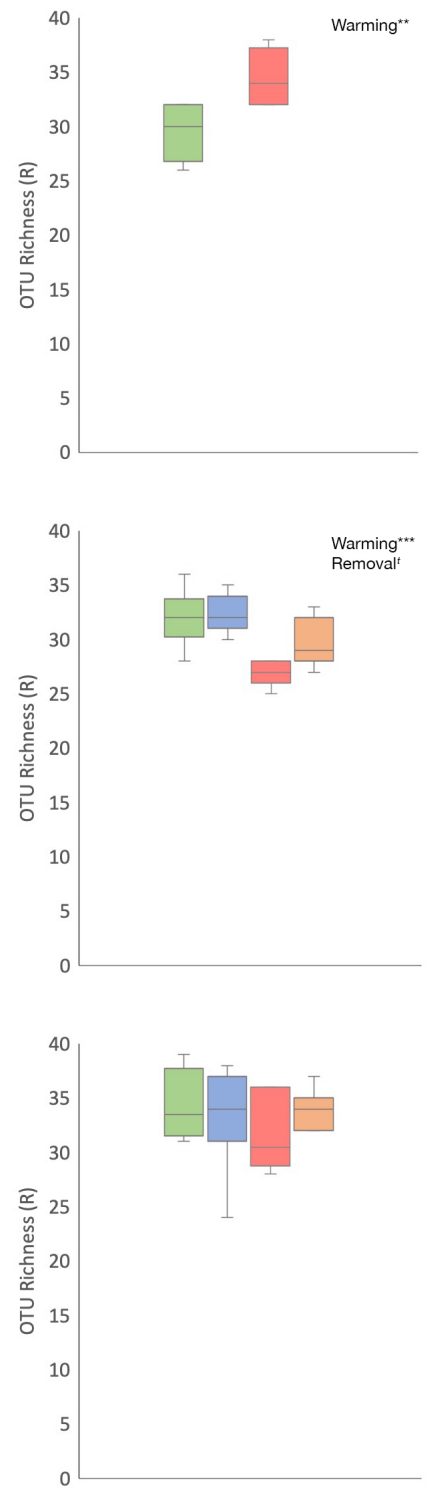

Pielou's Evenness
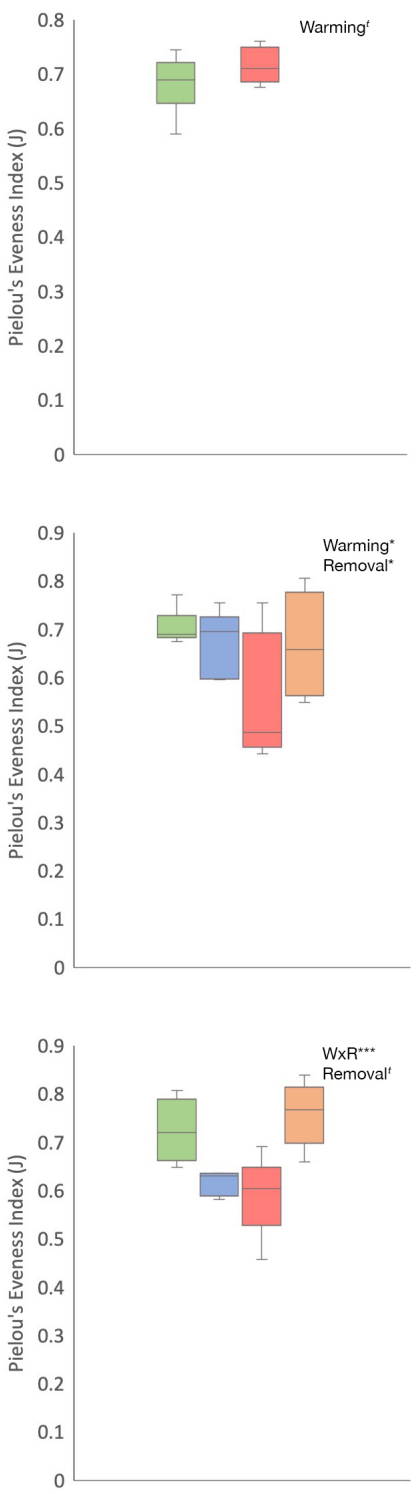

$\square$ ambient-control $\square$ ambient-removal $\square$ warm-control $\square$ warm-removal

Figure 18. Diversity indices (Shannon's Diversity, Richness, and Pielou's Evenness) of ErM OTUs associated with three ericoid mycorrhizal host plant species in response to factorial warming and removal treatment ( $\mathrm{n}=8$ per treatment, 32 total). Median values for each metric are denoted by a horizontal line, boxes represent the interquartile ranges and whiskers denote the upper and lower 95\% confidence intervals for each metric in response to the four treatments. Significant treatment effects from the Two-Way ANOVA are noted with $* * *$ if $\mathrm{p}<0.0005, * *$ if $\mathrm{p}<0.005, *$ if $\mathrm{p}<0.05$. CALVUL, Calluna vulgaris. DRASUB, Dracophyllum subulatum. EPAALP, Epacris alpina. WxR, Warming and Removal factors as a significant treatment effect. 
Diversity analyses revealed that warming increased the diversity of ErM OTUs associated with $C$. vulgaris (Figure 18). C. vulgaris saw a significant increase in both Shannon diversity $(\mathrm{F}=9.16, \mathrm{p}=0.011)$, OTU richness $(\mathrm{F}=12.32, \mathrm{p}=0.004)$ due to warming, with a slight change in taxonomic distinctness (Peilou's Evenness) $(\mathrm{F}=3.44 \mathrm{p}$ $=0.08)$. In addition, ErM OTU richness was highest within C. vulgaris, particularly within the warm-control treatment, confirming that C. vulgaris is an ErM generalist, where the lower OTU richness reported in the MF OTU rarefaction curve, is likely a result of AMF exclusion and a lack thereof in the native ErM host plants.

In contrast, the diversity of the mycorrhizal community associated with other ErM hosts varied significantly in response to WaRM treatments. In D. subulatum, OTU diversity indices declined significantly with warming treatment, while C. vulgaris removal often increased mycorrhizal OTU diversity in D. subulatum. This led to significant interactions among warming and removal treatments for Shannon's diversity. In E. alpina, OTU richness was unchanged by the WaRM treatments, while indices that took into account OTU evenness among treatments both showed strong interactive effects of the warming and removal treatments. Warming and Removal independently both led to significant reductions in OTU evenness, but in combination tended to increase OTU evenness, relative to the control. All two-way ANOVA results of ErM host plant species are shown in Table 3. 
Table 3. Two-Way ANOVA results of ErM fungal OTU diversity (Shannon Diversity Index, Species Richness and Pielou's Evenness) in ErM host plant species. CALVUL, Calluna vulgaris. DRASUB, Dracophyllum subulatum. EPAALP, Epacris alpina. Treatment effects from the Two-Way ANOVA are denoted as an increase with $(+)$ or (-) with significance noted as $* * *$ if $\mathrm{p}<0.0005, * *$ if $\mathrm{p}<0.005, *$ if $\mathrm{p}<0.05,{ }^{t}$ if $\mathrm{p}<0.10$.

\begin{tabular}{|c|c|c|c|c|}
\hline INDEX & SPECIES & WARMING & REMOVAL & WxR \\
\hline \multirow{3}{*}{$\begin{array}{l}\text { SHANNON } \\
\text { DIVERSITY }\end{array}$} & CALVUL & $\begin{array}{l}\mathrm{F}=9.16 \\
\mathrm{p}=0.011 * * \\
(+)\end{array}$ & N/A & N/A \\
\hline & DRASUB & $\begin{array}{l}\mathrm{F}=9.51 \\
\mathrm{p}=0.005^{* *} \\
(-)\end{array}$ & $\begin{array}{l}F=2.38 \\
p=0.136\end{array}$ & $\begin{array}{l}F=6.54 \\
p=0.017 *\end{array}$ \\
\hline & EPAALP & $\begin{array}{l}F=0.15 \\
p=0.705\end{array}$ & $\begin{array}{l}F=2.86 \\
p=0.106\end{array}$ & $\begin{array}{l}\mathrm{F}=27.86 \\
\mathrm{p}=3.64 \mathrm{e}-05^{* * *}\end{array}$ \\
\hline \multirow{3}{*}{$\begin{array}{l}\text { SPECIES } \\
\text { RICHNESS }\end{array}$} & CALVUL & $\begin{array}{l}\mathrm{F}=12.32 \\
\mathrm{p}=0.004^{* * *} \\
(+)\end{array}$ & N/A & N/A \\
\hline & DRASUB & $\begin{array}{l}\mathrm{F}=22.73 \\
\mathrm{p}=6.8 \mathrm{e}-05^{* * *} \\
(-)\end{array}$ & $\begin{array}{l}\mathrm{F}=3.04 \\
\mathrm{p}=0.0936^{t} \\
(+)\end{array}$ & $\begin{array}{l}F=1.49 \\
p=0.2343\end{array}$ \\
\hline & EPAALP & $\begin{array}{l}F=0.241 \\
p=0.629\end{array}$ & $\begin{array}{l}F=0.356 \\
p=0.558\end{array}$ & $\begin{array}{l}F=1.231 \\
p=0.280\end{array}$ \\
\hline \multirow{3}{*}{$\begin{array}{l}\text { PEILOU'S } \\
\text { EVENNESS }\end{array}$} & CALVUL & $\begin{array}{l}\mathrm{F}=3.44 \\
\mathrm{p}=0.08^{t} \\
(+)\end{array}$ & N/A & N/A \\
\hline & DRASUB & $\begin{array}{l}\mathrm{F}=5.98 \\
\mathrm{p}=0.022 * \\
(-)\end{array}$ & $\begin{array}{l}F=1.935 \\
p=0.176\end{array}$ & $\begin{array}{l}F=6.112 \\
p=0.021 *\end{array}$ \\
\hline & EPAALP & $\begin{array}{l}F=0.173 \\
p=0.682\end{array}$ & $\begin{array}{l}\mathrm{F}=3.408 \\
\mathrm{p}=0.0799^{t} \\
(+)\end{array}$ & $\begin{array}{l}\mathrm{F}=8.86 \\
\mathrm{p}<0.001 * * *\end{array}$ \\
\hline
\end{tabular}



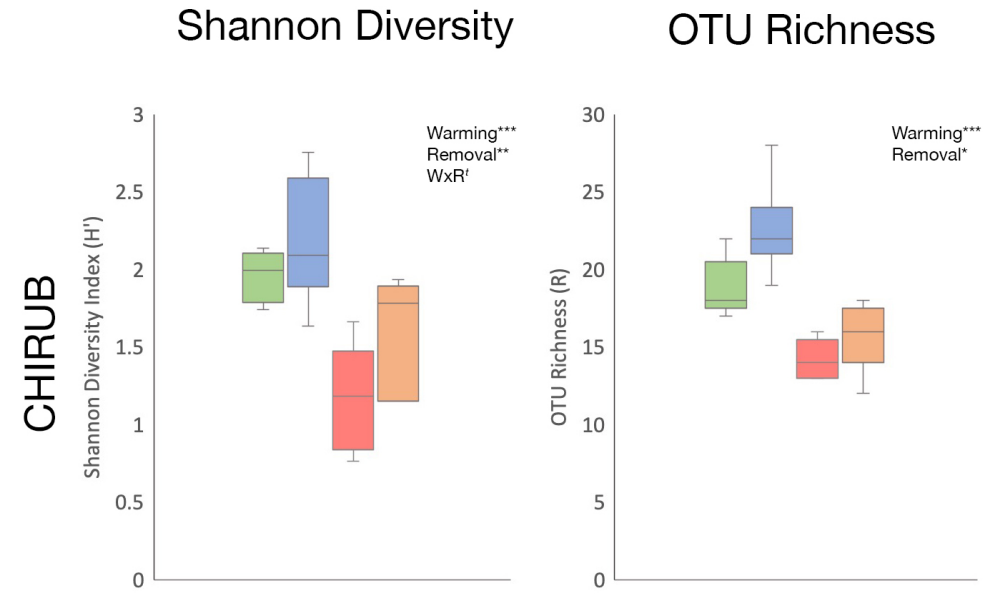

Pielou's Evenness
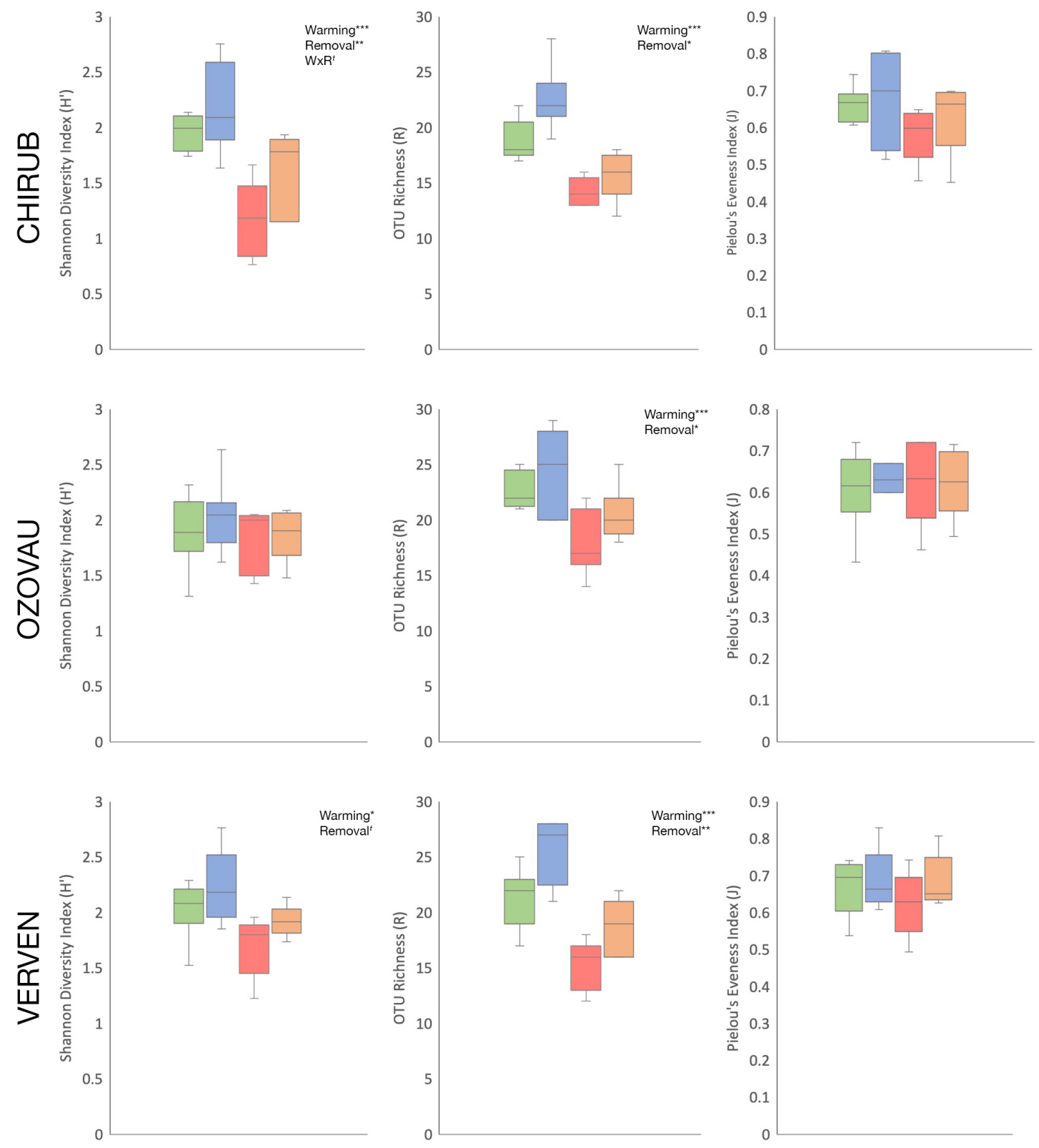

$\square$ ambient-control $\square$ ambient-removal $\square$ warm-control $\square$ warm-removal

Figure 19. Diversity indices (Shannon's Diversity, Richness, and Pielou's Evenness) of AM OTUs associated with three arbuscular mycorrhizal host plant species in response to factorial warming and removal treatment $(n=8$ per treatment, 32 total). Median values for each metric are denoted by a horizontal line, boxes represent the interquartile ranges and whiskers denote the upper and lower 95\% confidence intervals for each metric in response to the four treatments. Significant treatment effects from the Two-Way ANOVA are noted with $* * *$ if $\mathrm{p}<0.0005, * *$ if $\mathrm{p}<0.005, *$ if $\mathrm{p}<0.05$. CHIRUB, Chionochloa rubra. OZOVAU, Ozothamnus vauvilliersii. VERVEN, Veronica venustula. 
Diversity analyses revealed that warming significantly reduced the AM OTU richness of all AM host plant species. Warming also led to significant declines in AM OTU Shannon's diversity index in C. rubra and V. venustula. Conversely, C. vulgaris removal significantly increased AMF OTU richness in all host plants and Shannon's diversity index of AM OTUs in C. rubra and $V$. venustula. Evenness of the mycorrhizal OTUs tended to not to respond to treatment (Figure 19). All two-way ANOVA results of AM host plant species are shown in Table 4. 
Table 4. Two-Way ANOVA results of AM fungal OTU diversity (Shannon Diversity Index, Species Richness and Pielou's Evenness) in AM host plant species. CHIRUB, Chionochloa rubra. OZOVAU, Ozothamnus vauvilliersii. VERVEN, Veronica venustula. Treatment effects from the Two-Way ANOVA are denoted as an increase with $(+)$ or (-) with significance noted as $* * *$ if $\mathrm{p}<0.0005, * *$ if $\mathrm{p}<0.005, *$ if $\mathrm{p}<0.05,{ }^{t}$ if $\mathrm{p}<0.10$.

\begin{tabular}{|c|c|c|c|c|}
\hline INDEX & SPECIES & WARMING & REMOVAL & WxR \\
\hline \multirow{3}{*}{$\begin{array}{l}\text { SHANNON } \\
\text { DIVERSITY }\end{array}$} & CHIRUB & $\begin{array}{l}\mathrm{F}=24.55 \\
\mathrm{p}<0.001 * * * \\
(-)\end{array}$ & $\begin{array}{l}F=10.07 \\
p=0.005^{* *} \\
(+)\end{array}$ & $\begin{array}{l}\mathrm{F}=3.26 \\
\mathrm{p}=0.086^{t}\end{array}$ \\
\hline & OZOVAU & $\begin{array}{l}F=1.10 \\
p=0.305\end{array}$ & $\begin{array}{l}F=0.79 \\
p=0.383\end{array}$ & $\begin{array}{l}F=0.23 \\
p=0.634\end{array}$ \\
\hline & VERVEN & $\begin{array}{l}F=6.74 \\
p=0.018^{*} \\
(-)\end{array}$ & $\begin{array}{l}\mathrm{F}=3.84 \\
\mathrm{p}=0.067 t \\
(+)\end{array}$ & $\begin{array}{l}F<0.00 \\
p=0.983\end{array}$ \\
\hline \multirow{3}{*}{$\begin{array}{l}\text { SPECIES } \\
\text { RICHNESS }\end{array}$} & CHIRUB & $\begin{array}{l}\mathrm{F}=27.70 \\
\mathrm{p}<0.001 * * * \\
(-)\end{array}$ & $\begin{array}{l}F=6.54 \\
p=0.019^{*} \\
(+)\end{array}$ & $\begin{array}{l}F=0.40 \\
p=0.532\end{array}$ \\
\hline & OZOVAU & $\begin{array}{l}\mathrm{F}=18.31 \\
\mathrm{p}<0.001 * * * \\
(-)\end{array}$ & $\begin{array}{l}F=4.57 \\
p=0.042 * \\
(+)\end{array}$ & $\begin{array}{l}F=0.166 \\
p=0.687\end{array}$ \\
\hline & VERVEN & $\begin{array}{l}\mathrm{F}=26.85 \\
\mathrm{p}<0.001 * * * \\
(-)\end{array}$ & $\begin{array}{l}F=11.29 \\
p=0.004 * * \\
(+)\end{array}$ & $\begin{array}{l}F=0.199 \\
p=0.661\end{array}$ \\
\hline \multirow{3}{*}{$\begin{array}{l}\text { PEILOU'S } \\
\text { EVENNESS }\end{array}$} & CHIRUB & $\begin{array}{l}F=3.12 \\
p=0.093^{t}(-)\end{array}$ & $\begin{array}{l}F=0.55 \\
p=0.468\end{array}$ & $\begin{array}{l}F=0.67 \\
p=0.424\end{array}$ \\
\hline & OZOVAU & $\begin{array}{l}F=0.05 \\
p=0.828\end{array}$ & $\begin{array}{l}F=0.08 \\
p=0.786\end{array}$ & $\begin{array}{l}F=0.58 \\
p=0.454\end{array}$ \\
\hline & VERVEN & $\begin{array}{l}F=0.89 \\
p=0.357\end{array}$ & $\begin{array}{l}F=0.90 \\
p=0.355\end{array}$ & $\begin{array}{l}F=0.02 \\
p=0.905\end{array}$ \\
\hline
\end{tabular}


Non-Metric Multidimensional Scaling Analysis

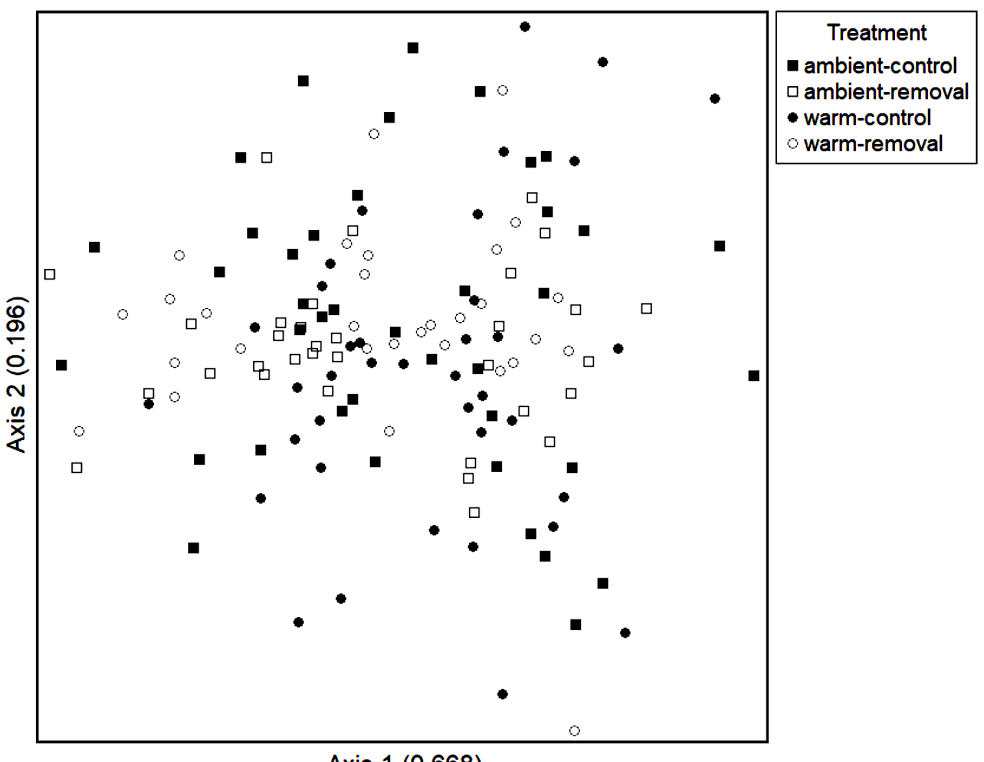

Axis $1(0.668)$

(A)

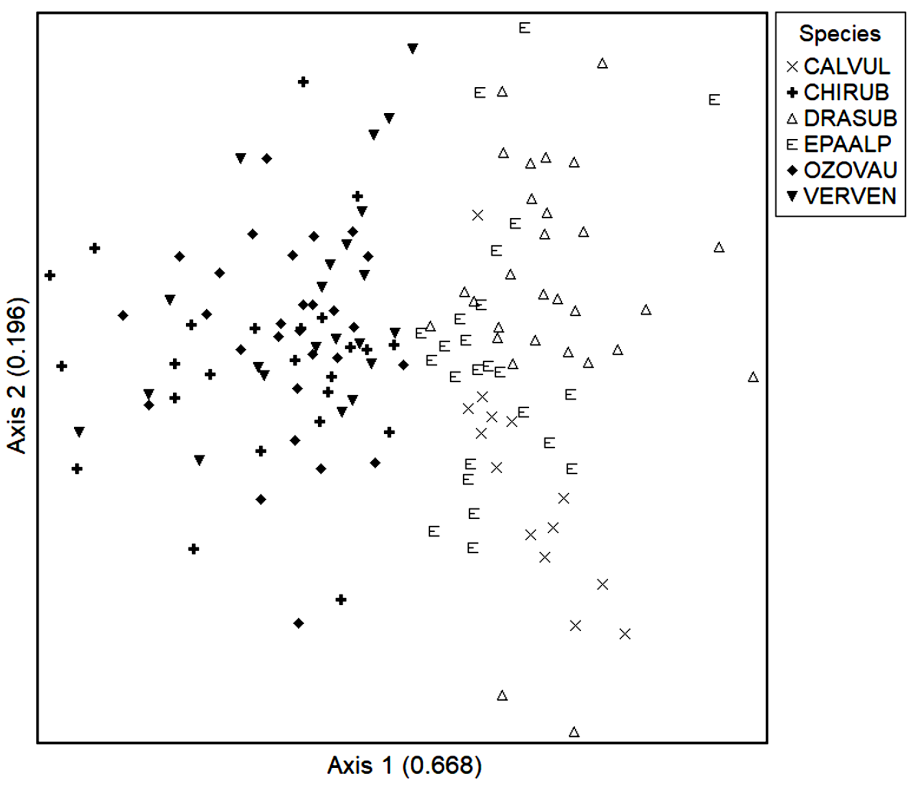

(B)

Figure 20. NMS graph with all plant root samples $(\mathbf{n}=\mathbf{1 4 1})$ arranged in mycorrhizal OTU (p= 100) ordination space by treatment (A) and by species (B). CALVUL, Calluna vulgaris CHIRUB, Chionochloa rubra. DRASUB, Dracophyllum subulatum. EPAALP, Epacris alpina. OZOVAU, Ozothamnus vauvilliersii. VERVEN, Veronica venustula

NMS ordination of all samples by all mycorrhizal OTUs described a high proportion of the total variance in mycorrhizal communities across samples (cumulative total of $86.4 \%$ of variance explained), but revealed no clear clustering of samples according to the WaRM treatments (Figure 20A). Nevertheless, MRPP revealed significant differences in OTU composition between the treatments $(A=0.01, p=0.002)$. There were also significant pairwise differences between the ambient-control and the singular treatments (Table 5). In stark contrast, when samples were labelled according to the plant species in the NMS ordination clear patterns emerge in the overall structure of the mycorrhizal OTUs (Figure 20B). Specifically, variation in the structure of 
mycorrhizal communities corresponded strongly to mycorrhizal type with AM host plants (C. rubra, O. vauvilliersii and $V$. venustula) assemblages differing starkly from those of ErM hosts (C. vulgaris, D. subulatum and E. alpina) along Axis 1, which accounts for $66.8 \%$ of the total variance in the dataset. Accordingly MRPP revealed and overall strong and significant effect of host plant species on the mycorrhizal OTU composition of samples $(A=0.159, p<0.001)$. There were also significant pairwise differences between all species which are listed in Table 6. Likewise, mycorrhizal type of the host plant species explained a significant proportion of the variance in fungal OTU composition among sample as determined by MRPP $(A=0.109, \mathrm{p}=0.005)$. MRPP also revealed that individual plot had no significant effect on mycorrhizal OTU composition $(\mathrm{A}=0.001, \mathrm{p}=$ $0.451)$.

Table 5. Pairwise differences of Multiple Response Permutation Procedure (MRPP) of all treatments $(\mathbf{n}=\mathbf{4})$ by mycorrhizal OTUs $(\mathbf{p}=\mathbf{1 0 0})$. Significant difference between treatments detected with MRPP are denoted with $* * *$ if $\mathrm{p}<0.0005, * *$ if $\mathrm{p}<0.005, *$ if $\mathrm{p}$ $<0.05,{ }^{t}$ if $\mathrm{p}<0.10$.

\begin{tabular}{c|ccc} 
TREATMENT & $\begin{array}{c}\text { AMBIENT } \\
\text { CONTROL }\end{array}$ & $\begin{array}{c}\text { AMBIENT } \\
\text { REMOVAL }\end{array}$ & $\begin{array}{c}\text { WARM } \\
\text { CONTROL }\end{array}$ \\
\hline AMBIENT & A $=0.006$ & \\
REMOVAL & $\mathrm{p}=0.046^{*}$ & & \\
& & & \\
WARM & $\mathrm{A}=0.005$ & $\mathrm{~A}=0.020$ & \\
CONTROL & $\mathrm{p}=0.041^{*}$ & $\mathrm{p}=0.004^{* *}$ & \\
& & & $\mathrm{~A}=0.003$ \\
WARM & $\mathrm{A}=0.002$ & $\mathrm{p}=0.167$ \\
REMOVAL & $\mathrm{p}=0.151$ & $\mathrm{~A}=0.006$ &
\end{tabular}


Table 6. Pairwise differences of Multiple Response Permutation Procedure (MRPP) of all host-plant species $(\mathbf{n}=\mathbf{6})$ by mycorrhizal OTUs $(\mathbf{p}=\mathbf{1 0 0})$. CALVUL, Calluna vulgaris CHIRUB, Chionochloa rubra. DRASUB, Dracophyllum subulatum. EPAALP, Epacris alpina. OZOVAU, Ozothamnus vauvilliersii. VERVEN, Veronica venustula. Significant difference between treatments detected with MRPP are denoted with $* * *$ if $\mathrm{p}<0.0005, * *$ if $\mathrm{p}<0.005, *$ if $\mathrm{p}<0.05,{ }^{t}$ if $\mathrm{p}<0.10$.

\begin{tabular}{|c|c|c|c|c|c|}
\hline SPECIES & CALVUL & CHIRUB & DRASUB & EPAALP & OZOVAU \\
\hline CHIRUB & $\begin{array}{c}A=0.163 \\
p<0.001 * * *\end{array}$ & & & & \\
\hline DRASUB & $\begin{array}{c}\mathrm{A}=0.063 \\
\mathrm{p}<0.001^{* * *}\end{array}$ & $\begin{array}{c}\mathrm{A}=0.132 \\
\mathrm{p}<0.001^{* * *}\end{array}$ & & & \\
\hline EPAALP & $\begin{array}{c}\mathrm{A}=0.061 \\
\mathrm{p}<0.001 * * *\end{array}$ & $\begin{array}{c}\mathrm{A}=0.123 \\
\mathrm{p}<0.001 * * *\end{array}$ & $\begin{array}{c}\mathrm{A}=0.033 \\
\mathrm{p}<0.001^{* * *}\end{array}$ & & \\
\hline OZOVAU & $\begin{array}{c}\mathrm{A}=0.170 \\
\mathrm{p}<0.001^{* * *}\end{array}$ & $\begin{array}{c}A=0.035 \\
p<0.001 * * *\end{array}$ & $\begin{array}{c}\mathrm{A}=0.139 \\
\mathrm{p}<0.001 * * *\end{array}$ & $\begin{array}{c}\mathrm{A}=0.133 \\
\mathrm{p}<0.001 * * *\end{array}$ & \\
\hline VERVEN & $\begin{array}{c}A=0.158 \\
p<0.001^{* * *}\end{array}$ & $\begin{array}{c}\mathrm{A}=0.031 \\
\mathrm{p}<0.001 * * *\end{array}$ & $\begin{array}{c}\mathrm{A}=0.119 \\
\mathrm{p}<0.001 * * *\end{array}$ & $\begin{array}{c}\mathrm{A}=0.116 \\
\mathrm{p}<0.001 * * *\end{array}$ & $\begin{array}{c}\mathrm{A}=0.045 \\
\mathrm{p}<0.001 * * *\end{array}$ \\
\hline
\end{tabular}




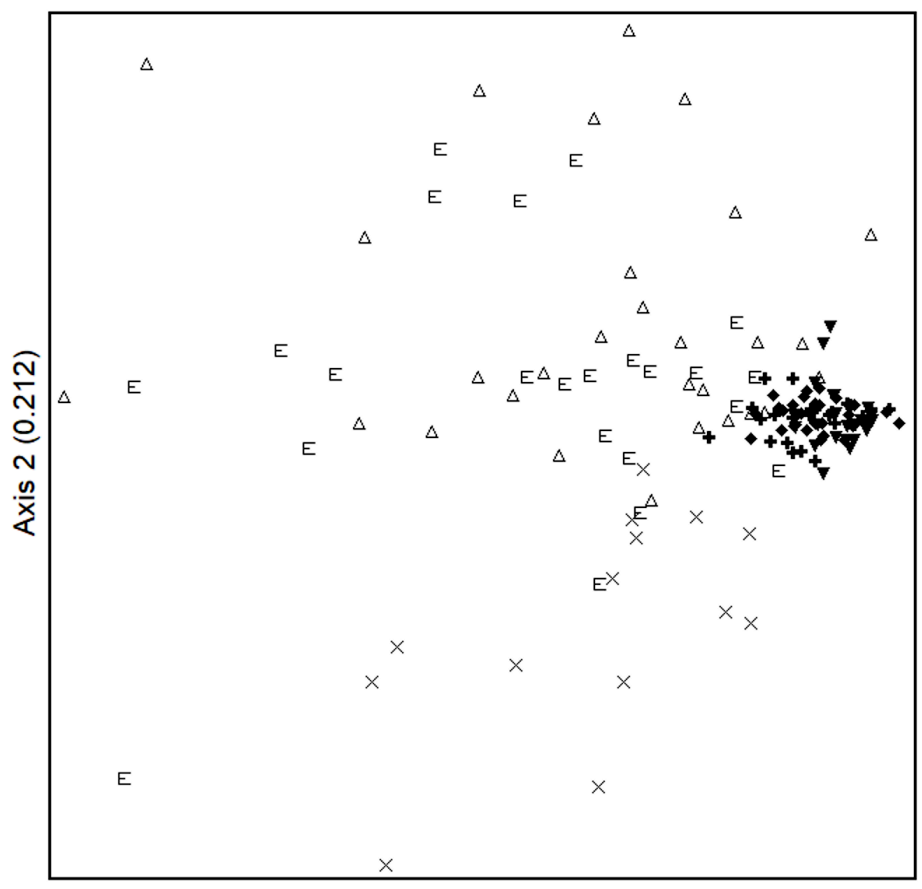

Axis $1(0.608)$

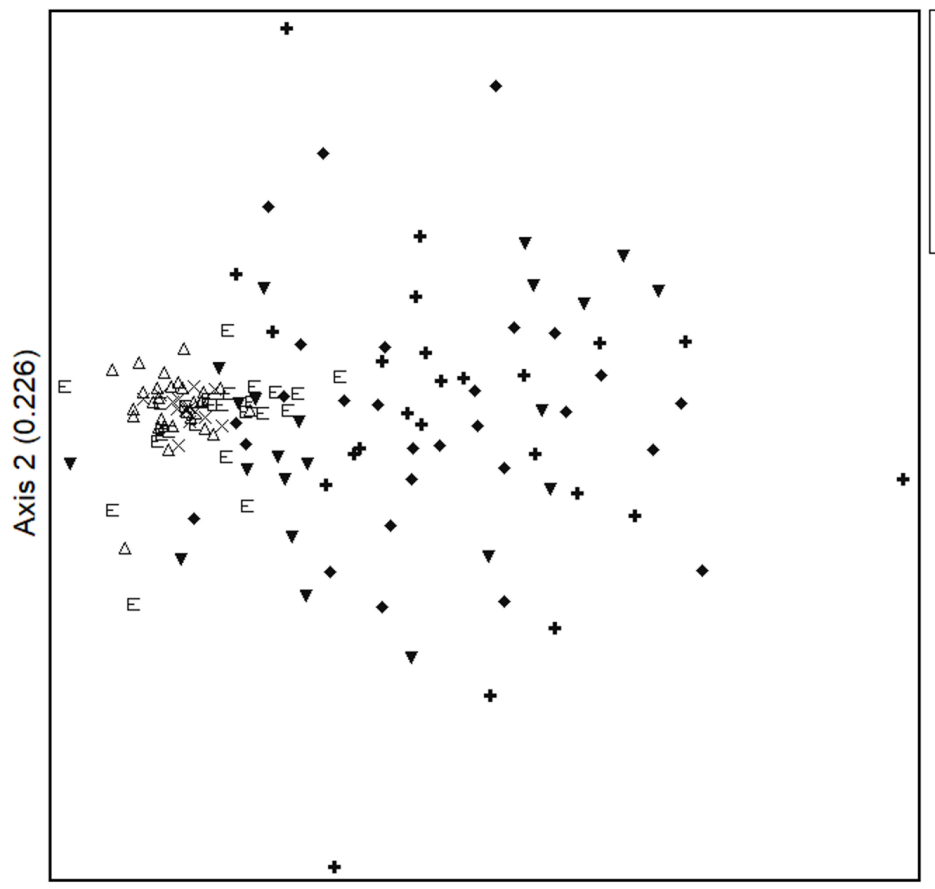

Axis $1(0.678)$

(B)

(A)

Figure 21. NMS graphs with all host-plant species $(\mathbf{n}=\mathbf{1 4 1})$ arranged in mycorrhizal OTU ordination space by ErM OTUs $(\mathbf{p}=\mathbf{4 7})(\mathbf{A})$ and AMF OTUs $(\mathbf{p}=\mathbf{5 3})$ (B). CALVUL, Calluna vulgaris CHIRUB, Chionochloa rubra. DRASUB, Dracophyllum subulatum. EPAALP, Epacris alpina. OZOVAU, Ozothamnus vauvilliersii. VERVEN, Veronica venustula.

NMS ordination of all samples by mycorrhizal OTU guild confirms that the main source of variation (Axis 1) is the mycorrhizal type of the host plant species for both the variance in ErM OTUs (Figure 21A) and AMF OTUs (Figure 21B), but revealed no clear clustering between species. In Figure 21A all samples were arranged by ErM OTU ordination space. Axis 1 describes $60.8 \%$ of the total variance within data, Axis 2 describes $21.2 \%$. Cumulatively, this graph describes $82 \%$ of the total variance found within the data. In Figure 21B all samples were arranged by AMF OTU ordination space. Axis 1 describes $67.8 \%$ of the total variance within data, Axis 2 describes $22.6 \%$. 
Cumulatively, this graph describes $90.4 \%$ of the total variance found within the data.

Overall, ErM host plant species had a significant effect on the composition of the mycorrhizal community, as determined by MRPP $(\mathrm{A}=0.168, \mathrm{p}<0.0001$, Figure 21A, Table 7). Likewise, AM host plant species had a significant effect on the composition of the mycorrhizal community overall, as determined by $\operatorname{MRPP}(\mathrm{A}=0.194, \mathrm{p}<0.001)$, but pairwise differences were not found for all species (Figure 21B, Table 8). For example, the AMF communities of $O$. vauvilliersii and C. rubra. did not differ significantly by MRPP.

Table 7. Pairwise differences of Multiple Response Permutation Procedure (MRPP) of all host-plant species $(\mathbf{n}=\mathbf{6})$ by ErM OTUs $(\mathbf{p}=\mathbf{4 7})$. CALVUL, Calluna vulgaris CHIRUB, Chionochloa rubra. DRASUB, Dracophyllum subulatum. EPAALP, Epacris alpina. OZOVAU, Ozothamnus vauvilliersii. VERVEN, Veronica venustula. Significant difference between treatments detected with MRPP are denoted with $* * *$ if $\mathrm{p}<0.0005, * *$ if $\mathrm{p}<0.005, *$ if $\mathrm{p}<0.05$.

\begin{tabular}{|c|c|c|c|c|c|}
\hline SPECIES & CALVUL & CHIRUB & DRASUB & EPAALP & OZOVAU \\
\hline CHIRUB & $\begin{array}{c}\mathrm{A}=0.163 \\
\mathrm{p}<0.001^{* * *}\end{array}$ & & & & \\
\hline DRASUB & $\begin{array}{c}\mathrm{A}=0.068 \\
\mathrm{p}<0.001 * * *\end{array}$ & $\begin{array}{c}\mathrm{A}=0.121 \\
\mathrm{p}<0.001^{* * *}\end{array}$ & & & \\
\hline EPAALP & $\begin{array}{c}\mathrm{A}=0.065 \\
\mathrm{p}<0.001 * * *\end{array}$ & $\begin{array}{c}\mathrm{A}=0.137 \\
\mathrm{p}<0.001 * * *\end{array}$ & $\begin{array}{c}\mathrm{A}=0.034 \\
\mathrm{p}<0.001 * * *\end{array}$ & & \\
\hline OZOVAU & $\begin{array}{c}\mathrm{A}=0.196 \\
\mathrm{p}<0.001 * * *\end{array}$ & $\begin{array}{l}A=0.139 \\
p=0.329\end{array}$ & $\begin{array}{c}\mathrm{A}=0.135 \\
\mathrm{p}<0.001 * * *\end{array}$ & $\begin{array}{c}\mathrm{A}=0.154 \\
\mathrm{p}<0.001^{* * *}\end{array}$ & \\
\hline VERVEN & $\begin{array}{c}\mathrm{A}=0.195 \\
\mathrm{p}<0.001 * * *\end{array}$ & $\begin{array}{c}\mathrm{A}=0.119 \\
\mathrm{p}<0.005^{* *}\end{array}$ & $\begin{array}{c}\mathrm{A}=0.132 \\
\mathrm{p}<0.001 * * *\end{array}$ & $\begin{array}{c}\mathrm{A}=0.150 \\
\mathrm{p}<0.001 * * *\end{array}$ & $\begin{array}{c}\mathrm{A}=0.011 \\
\mathrm{p}<0.001 * * *\end{array}$ \\
\hline
\end{tabular}


Table 8. Pairwise differences of Multiple Response Permutation Procedure (MRPP) of all host-plant species $(\mathbf{n}=\mathbf{6})$ by AMF OTUs $(\mathbf{p}=\mathbf{5 3})$. CALVUL, Calluna vulgaris CHIRUB, Chionochloa rubra. DRASUB, Dracophyllum subulatum. EPAALP, Epacris alpina. OZOVAU, Ozothamnus vauvilliersii. VERVEN, Veronica venustula. Significant difference between treatments detected with MRPP are denoted with $* * *$ if $\mathrm{p}<0.0005, * *$ if $\mathrm{p}<0.005, *$ if $\mathrm{p}<0.05$.

\begin{tabular}{|c|c|c|c|c|c|}
\hline SPECIES & CALVUL & CHIRUB & DRASUB & EPAALP & OZOVAU \\
\hline CHIRUB & $\begin{array}{c}\mathrm{A}=0.215 \\
\mathrm{p}<0.001 * * *\end{array}$ & & & & \\
\hline DRASUB & $\begin{array}{l}A=0.002 \\
p=0.229\end{array}$ & $\begin{array}{c}\mathrm{A}=0.224 \\
\mathrm{p}<0.001 * * *\end{array}$ & & & \\
\hline EPAALP & $\begin{array}{c}\mathrm{A}=0.033 \\
\mathrm{p}<0.001^{* * *}\end{array}$ & $\begin{array}{c}\mathrm{A}=0.154 \\
\mathrm{p}<0.001 * * *\end{array}$ & $\begin{array}{c}\mathrm{A}=0.029 \\
\mathrm{p}<0.001^{* * *}\end{array}$ & & \\
\hline OZOVAU & $\begin{array}{c}\mathrm{A}=0.197 \\
\mathrm{p}<0.001 * * *\end{array}$ & $\begin{array}{c}\mathrm{A}=0.049 \\
\mathrm{p}<0.001 * * *\end{array}$ & $\begin{array}{c}\mathrm{A}=0.212 \\
\mathrm{p}<0.001^{* * *}\end{array}$ & $\begin{array}{c}\mathrm{A}=0.149 \\
\mathrm{p}<0.001 * * *\end{array}$ & \\
\hline VERVEN & $\begin{array}{c}\mathrm{A}=0.156 \\
\mathrm{p}<0.001 * * *\end{array}$ & $\begin{array}{c}\mathrm{A}=0.042 \\
\mathrm{p}<0.001^{* * *}\end{array}$ & $\begin{array}{c}\mathrm{A}=0.152 \\
\mathrm{p}<0.001 * * *\end{array}$ & $\begin{array}{c}\mathrm{A}=0.084 \\
\mathrm{p}<0.001^{* * *}\end{array}$ & $\begin{array}{c}\mathrm{A}=0.069 \\
\mathrm{p}<0.001 * * *\end{array}$ \\
\hline
\end{tabular}




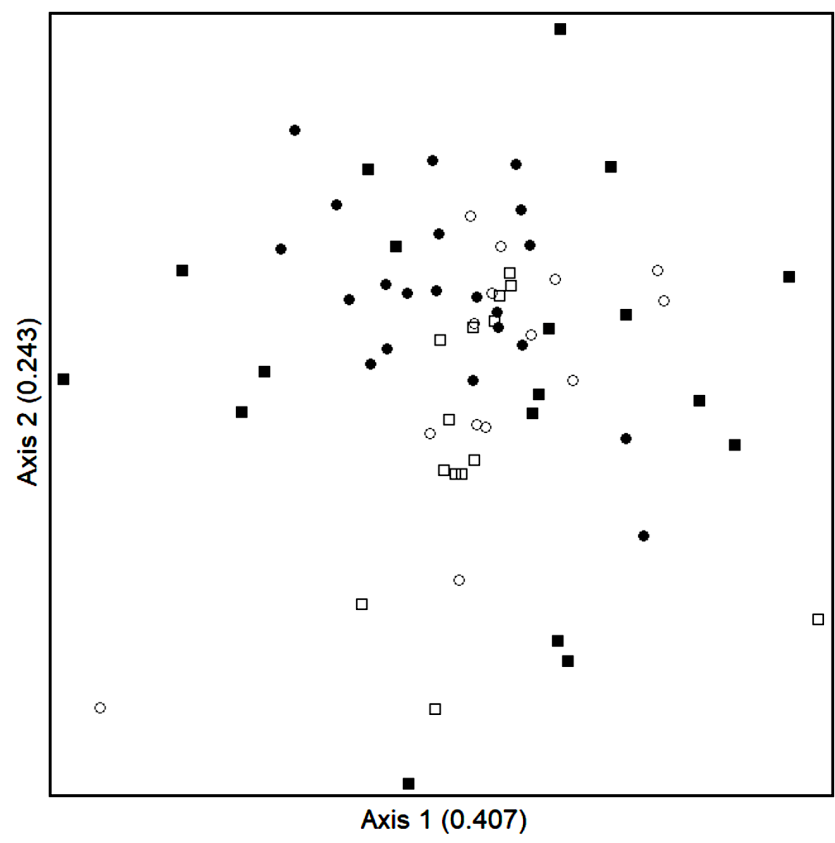

(A)

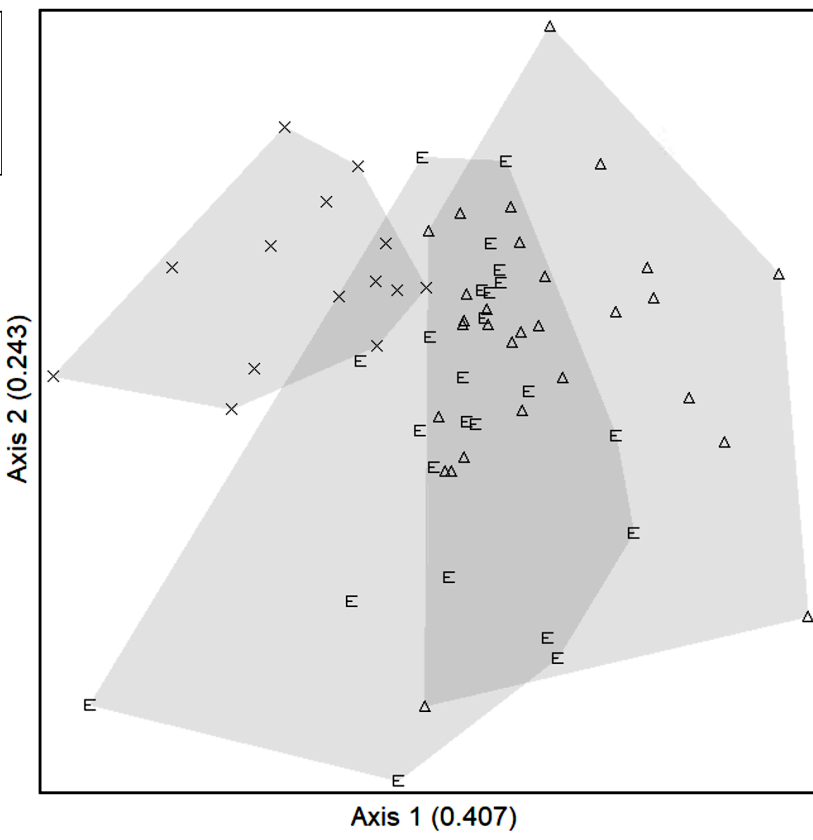

(B)

Figure 22. NMS graphs with all host plant samples that form ericoid mycorrhizal associations $(\mathbf{n}=\mathbf{6 7})$ arranged in ericoid mycorrhizal $($ ErM $)$ fungi OTU $(\mathbf{p}=\mathbf{4 7})$ ordination space by treatment (A) and by species (B). CALVUL, Calluna vulgaris. DRASUB, Dracophyllum subulatum. EPAALP, Epacris alpina.

In the ordination of ErM host plant samples by ErM OTUs (Figure 22), Axis 1 describes $40.7 \%$ of the total variance within data, Axis 2 describes $24.3 \%$. Cumulatively this graph describes $65.0 \%$ of the total variance found within the data. In Figure $22 \mathrm{~A}$, there is no-visual indication of clustering due to treatment effect, however MRPP found significant difference in OTU variance between the treatments $(\mathrm{A}=0.020, \mathrm{p}<0.001)$ with pairwise differences listed in Table 9. However, warm-removal treatment had no significant differences in ErM OTU variance, compared to the other three treatments. Conversely, MRPP found significant difference in ErM OTU composition between all species $(A=0.068, p<0.001)$ with pairwise differences listed in Table 10. Figure 22B also confirms species-specific ErM OTU assemblages, with a largest disparity between $C$. vulgaris and $c$. This figure also suggests an overlap of functional niche as an ErM host by 
E. alpina of the two other hosts. MRPP also revealed that individual plot had no significant effect on ErM OTU composition $(\mathrm{A}<0.001, \mathrm{p}=0.492)$.

Table 9. Pairwise differences of Multiple Response Permutation Procedure (MRPP) of all ErM host-plant species by treatment $(\mathbf{n}=\mathbf{4})$ by ErM OTUs $(\mathbf{p}=\mathbf{4 7 )}$ CALVUL, Calluna vulgaris. DRASUB, Dracophyllum subulatum. EPAALP, Epacris alpina. Significant difference between treatments detected with MRPP are denoted with *** if $\mathrm{p}<0.0005, * *$ if $\mathrm{p}<0.005, *$ if $\mathrm{p}<0.05$.

\begin{tabular}{c|ccc} 
TREATMENT & $\begin{array}{c}\text { AMBIENT } \\
\text { CONTROL }\end{array}$ & $\begin{array}{c}\text { AMBIENT } \\
\text { REMOVAL }\end{array}$ & $\begin{array}{c}\text { WARM } \\
\text { CONTROL }\end{array}$ \\
\hline AMBIENT & $\begin{array}{c}\mathrm{A}=0.016 \\
\mathrm{p}=0.014^{* *}\end{array}$ & & \\
REMOVAL & $\mathrm{A}=0.017$ & & \\
WARM & $\mathrm{p}=0.004^{* *}$ & $\mathrm{~A}=0.023$ & \\
CONTROL & & $\mathrm{p}=0.001^{* *}$ & \\
WARM & $\mathrm{A}=0.010$ & $\mathrm{~A}=0.009$ & $\mathrm{~A}=0.001$ \\
REMOVAL & $\mathrm{p}=0.056$ & $\mathrm{p}=0.117$ &
\end{tabular}

Table 10. Pairwise differences of Multiple Response Permutation Procedure (MRPP) between ErM host-plant species $(\mathbf{n}=\mathbf{3})$ by ErM OTUs $(\mathbf{p}=\mathbf{5 3})$. CALVUL, Calluna vulgaris. DRASUB, Dracophyllum subulatum. EPAALP, Epacris alpina. Significant difference between treatments detected with MRPP are denoted with *** if $\mathrm{p}<0.0005$, ** if $\mathrm{p}<0.005, *$ if $\mathrm{p}<0.05$.

\begin{tabular}{c|cc} 
SPECIES & CALVUL & DRASUB \\
\hline DRASUB & $\mathrm{A}=0.068$ \\
& $\mathrm{p}<0.001^{* * *}$ & \\
EPAALP & $\mathrm{A}=0.065$ & $\mathrm{~A}=0.034$ \\
& $\mathrm{p}<0.001^{* * *}$ & $\mathrm{p}<0.001^{* * *}$
\end{tabular}




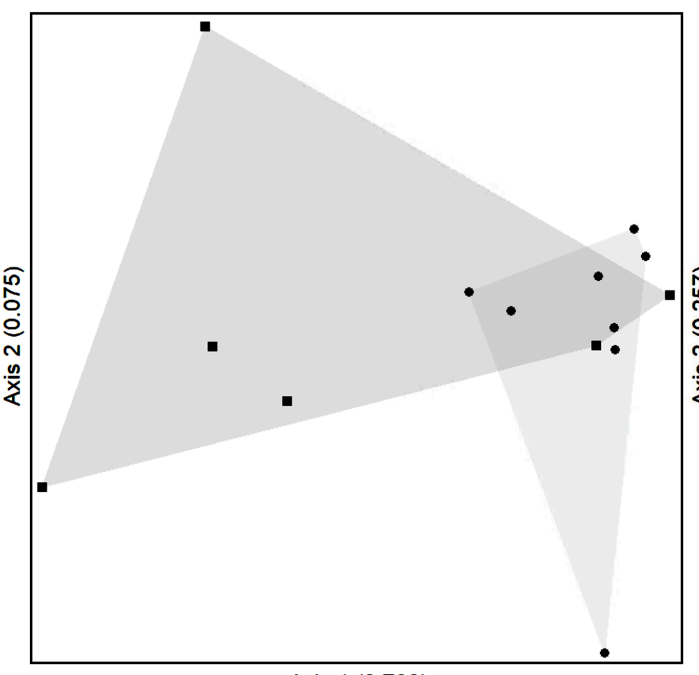

Axis $1(0.700)$

(A)

CALVUL

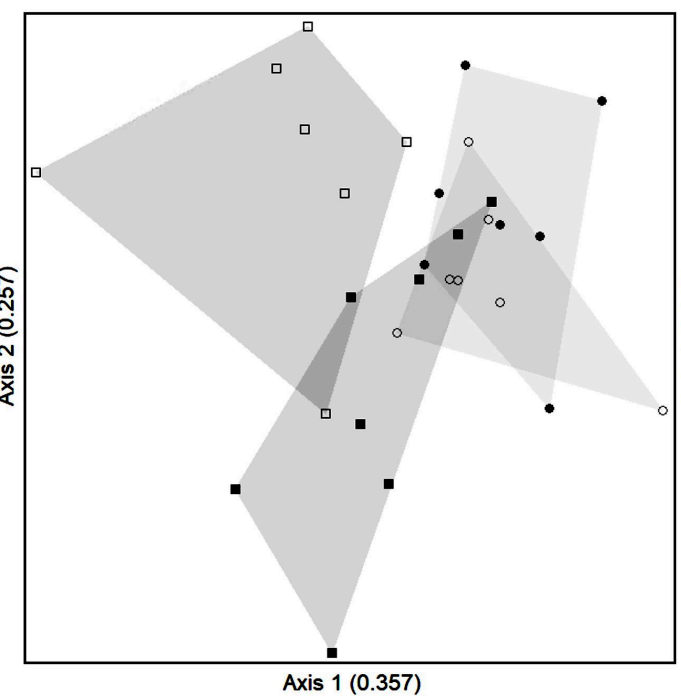

(B)

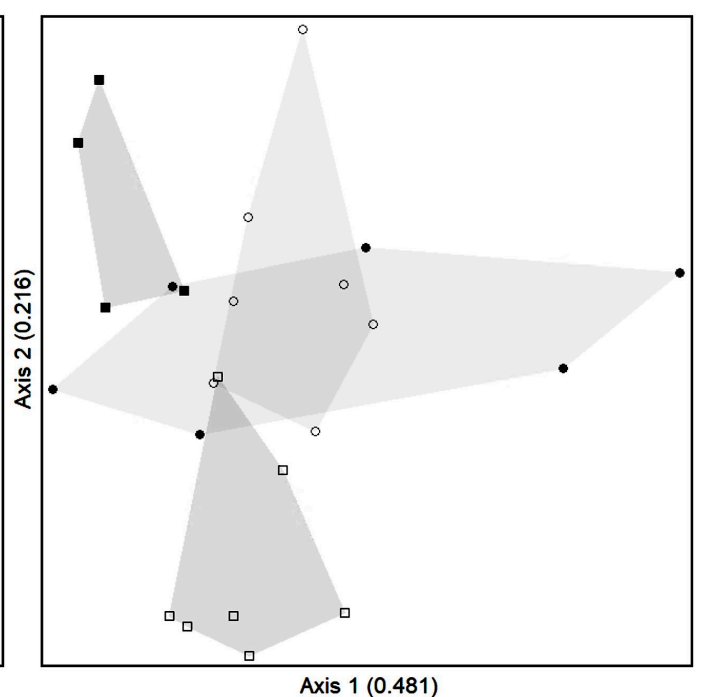

(C)

DRASUB

\section{Treatment}

- ambient-control $\square$ ambient-removal warm-control o warm-removal

Figure 23. NMS graphs by host plant species that form ericoid mycorrhizal associations, CALVUL, Calluna vulgaris $(\mathbf{n}=\mathbf{1 4})(\mathbf{A})$, DRASUB, Dracophyllum subulatum $(\mathbf{n}=\mathbf{2 9})$ (B), EPAALP, Epacris alpina $(\mathbf{n}=\mathbf{2 4})(\mathbf{C})$, arranged in ericoid mycorrhizal ( ErM ) fungi OTU $(\mathbf{p}=\mathbf{4 7})$ ordination space by treatment. Axes are labeled as the proportion of variation explained, all MRPP pairwise differences are listed in Appendix 1, Tables 2-3.

MRPP found significant difference in ErM OTU variance between treatments for all ErM host plants (Figure 23), C. vulgaris $(\mathrm{A}=0.042, \mathrm{p}=0.016)$, D. subulatum $(\mathrm{A}=$ $0.038, \mathrm{p}=0.016$, Appendix 1, Table 2), E. alpina $(\mathrm{A}=0.058, \mathrm{p}=0.002$, Appendix 1, Table 3). All ErM host plants had a significant difference in ErM OTU variance due to warming, however, D. subulatum and E. alpina had opposing responses to removal treatment. In D. subulatum there was no significant difference in the ErM OTU variance within the warmed plots. Whereas, in E. alpina there was no significant difference in the ErM OTU variance between the warm-control treatment and either of the removal plots. 


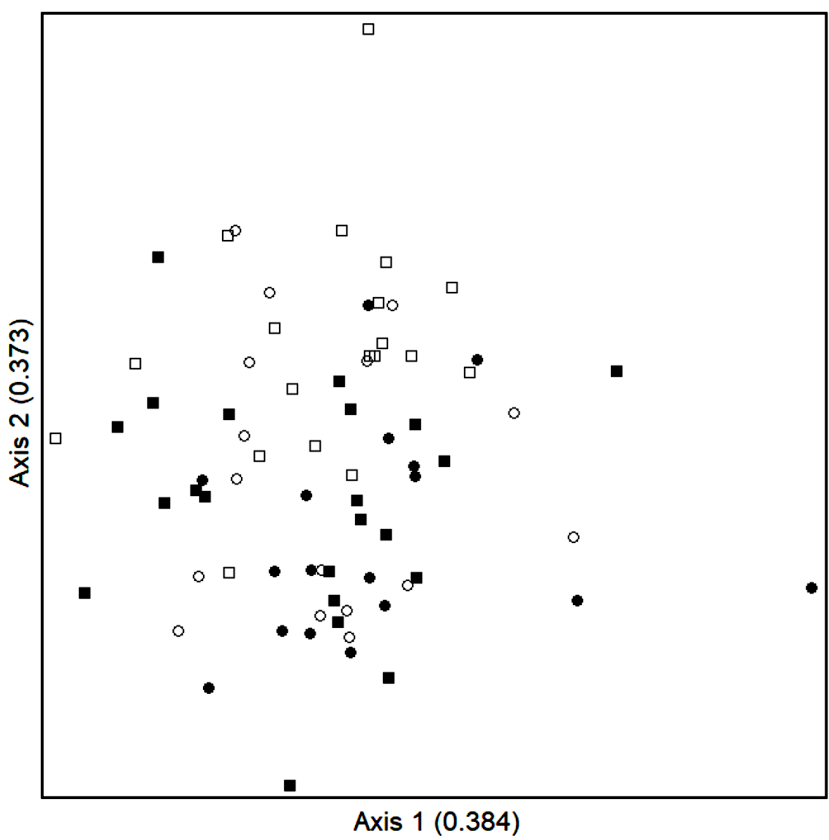

(A)

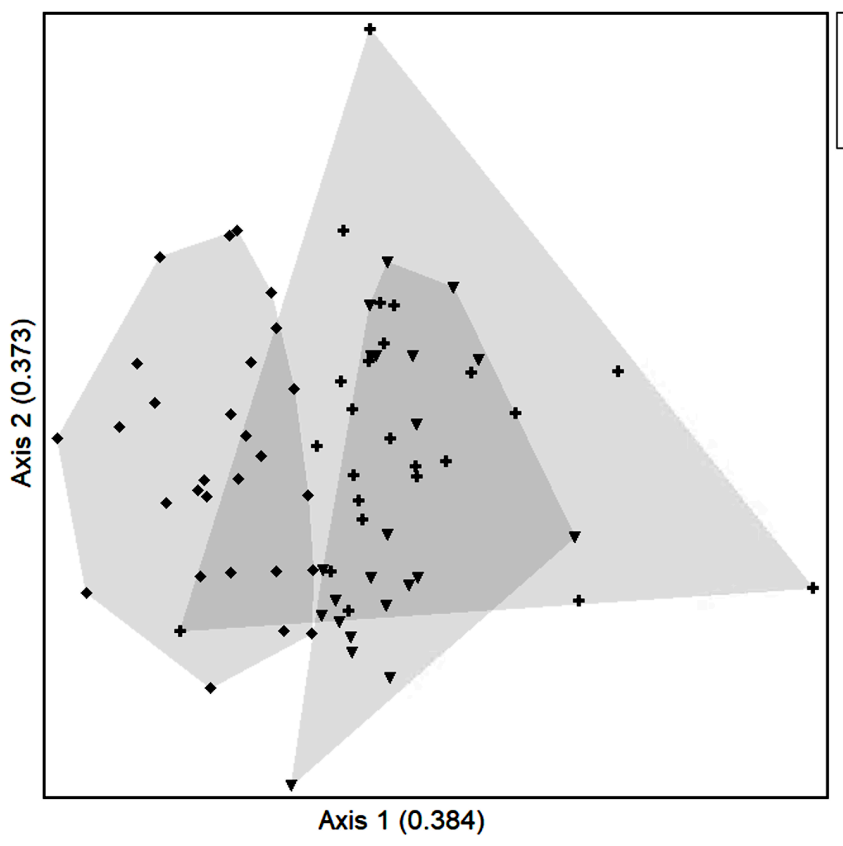

(B)

Figure 24. NMS graphs with all host plant samples that form arbuscular mycorrhizal associations $(\mathbf{n}=\mathbf{7 4})$ arranged in arbuscular mycorrhizal $(\mathrm{AM})$ fungi OTU (p = 53) ordination space by treatment (A) and by species (B) CHIRUB, Chionochloa rubra. OZOVAU, Ozothamnus vauvilliersii. VERVEN, Veronica venustula.

In the ordination of AM host plant samples by AMF OTUs (Figure 24), Axis 1 describes $38.4 \%$ of the total variance within data, Axis 2 describes $37.3 \%$. Cumulatively this graph describes $75.7 \%$ of the total variance found within the data. In Figure $24 \mathbf{A}$, there is no-visual indication of clustering due to treatment effect, however MRPP found significant difference in OTU variance between the treatments $(\mathrm{A}=0.036, \mathrm{p}<0.001)$ with pairwise differences listed in Table 11. However, ambient-removal was the only treatment that was detected to have a significant difference in AMF OTU variance between other treatments. Conversely, MRPP found significant difference in AM OTU composition between all species $(\mathrm{A}=0.063, \mathrm{p}<0.001)$. Figure 24B also confirms species-specific AM OTU assemblages, with a largest disparity between $O$. vauvilliersii and $V$. venustula, as listed in Table 12. This figure also suggests an overlap of functional 
niche as an AM host by C. rubra of the two other hosts. MRPP also revealed that individual plot had no significant effect on AM OTU composition $(\mathrm{A}<0.001, \mathrm{p}=0.543)$.

Table 11. Pairwise differences of Multiple Response Permutation Procedure (MRPP) of AM host-plant species by treatment $(\mathbf{n}=\mathbf{4})$ by AMF OTUs $(\mathbf{p}=\mathbf{5 3})$ CHIRUB, Chionochloa rubra. OZOVAU, Ozothamnus vauvilliersii. VERVEN, Veronica venustula. Significant difference between treatments detected with MRPP are denoted with $* * *$ if $\mathrm{p}$ $<0.0005, * *$ if $\mathrm{p}<0.005, *$ if $\mathrm{p}<0.05$.

\begin{tabular}{c|ccc} 
TREATMENT & $\begin{array}{c}\text { AMBIENT } \\
\text { CONTROL }\end{array}$ & $\begin{array}{c}\text { AMBIENT } \\
\text { REMOVAL }\end{array}$ & $\begin{array}{c}\text { WARM } \\
\text { CONTROL }\end{array}$ \\
\hline AMBIENT & $\begin{array}{c}\mathrm{A}=0.039 \\
\mathrm{p}<0.001^{* * *}\end{array}$ & & \\
REMOVAL & & & \\
& $\mathrm{A}=0.005$ & $\mathrm{~A}=0.061$ & \\
WARM & $\mathrm{p}=0.164$ & $\mathrm{p}<0.001^{* * *}$ & \\
CONTROL & & $\mathrm{A}=0.030$ & $\mathrm{~A}=0.005$ \\
& $\mathrm{p}=0.001$ & $\mathrm{p}=0.003^{* * *}$ & \\
WARM & $\mathrm{p}=0.352$ & &
\end{tabular}

Table 12. Pairwise differences of Multiple Response Permutation Procedure (MRPP) between all AM host-plant species $(\mathbf{n}=\mathbf{3})$ by AMF OTUs $(\mathbf{p}=\mathbf{5 3})$. CHIRUB, Chionochloa rubra. OZOVAU, Ozothamnus vauvilliersii. VERVEN, Veronica venustula. Significant difference between treatments detected with MRPP are denoted with $* * *$ if $\mathrm{p}$ $<0.0005, * *$ if $\mathrm{p}<0.005, *$ if $\mathrm{p}<0.05$.

\begin{tabular}{c|cc} 
SPECIES & CHIRUB & OZOVAU \\
\hline OZOVAU & $\mathrm{A}=0.049$ & \\
& $\mathrm{p}<0.001^{* * *}$ & \\
VERVEN & $\mathrm{A}=0.034$ & $\mathrm{~A}=0.006$ \\
& $\mathrm{p}=0.001^{* * *}$ & $\mathrm{p}<0.001^{* * *}$
\end{tabular}




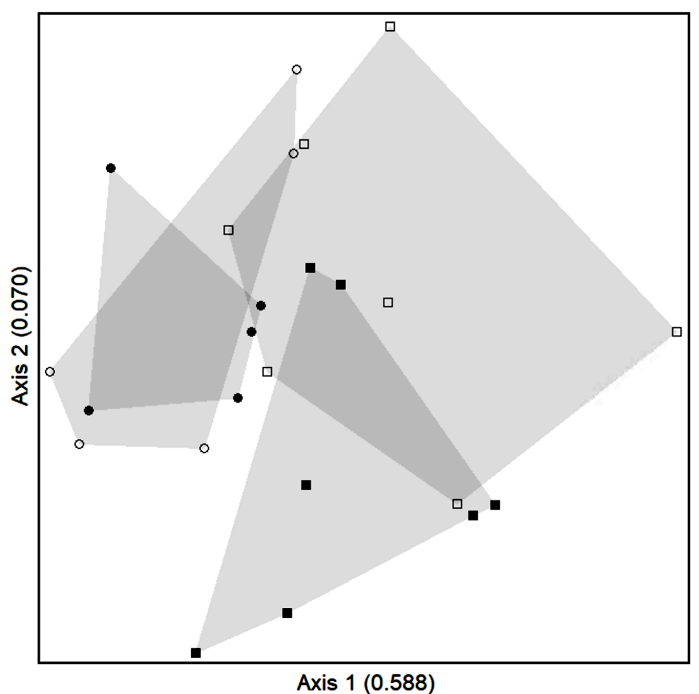

(A)

CHIRUB

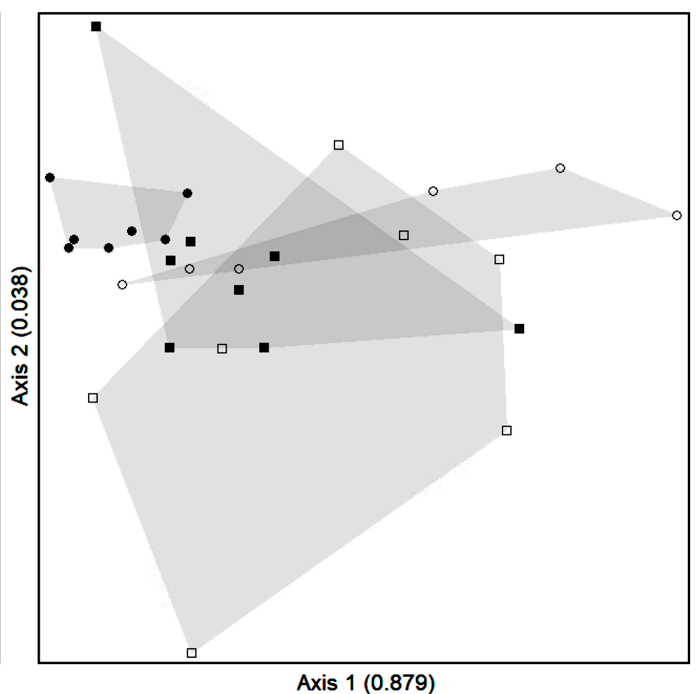

(B)

OZOVAU

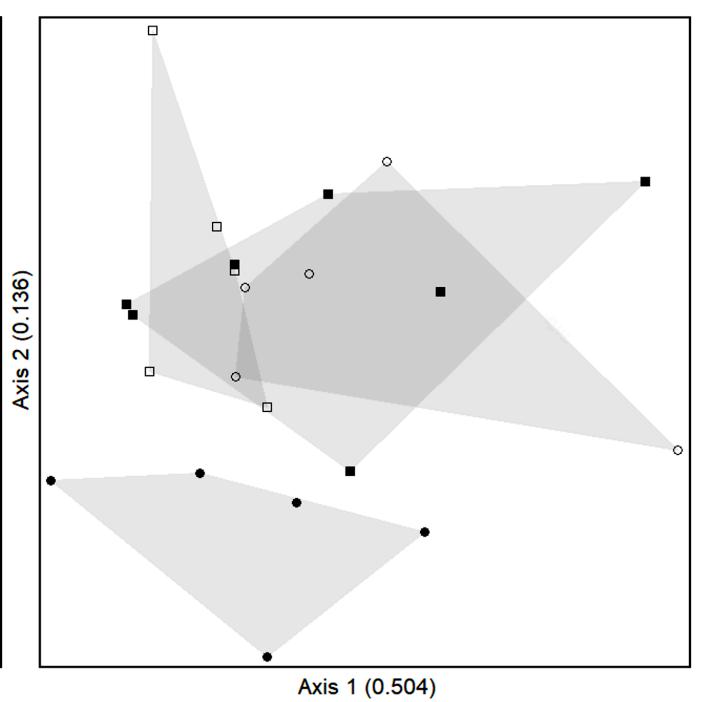

(C)

\section{Treatment}

- ambient-control $\square$ ambient-removal warm-control o warm-removal

Figure 25. NMS graphs by host plant species that form arbuscular mycorrhizal associations, CHIRUB, Chionochloa rubra (n = 24) (A), OZOVAU, Ozothamnus vauvilliersii $(\mathbf{n}=\mathbf{2 8})(\mathbf{B})$, VERVEN, Veronica venustula $(\mathbf{n}=\mathbf{2 2})(\mathbf{C})$ arranged in arbuscular mycorrhizal (AM) fungi OTU $(\mathbf{p}=\mathbf{5 3})$ ordination space by treatment. Axes are labeled as the proportion of variation explained, all MRPP pairwise differences are listed in Appendix 1. Tables 4-6.

MRPP found a significant difference in AM OTU variance between treatments for all AM host plants (Figure 25), C. rubra $(\mathrm{A}=0.071, \mathrm{p}=0.002$, Appendix 1. Table 4), $O$. vauvilliersii $(\mathrm{A}=0.130, \mathrm{p}=0.005$, Appendix 1. Table 5), V. venustula $(\mathrm{A}=0.056, \mathrm{p}<$ 0.001, Appendix 1. Table 6). All AM host plants had a significant difference in AM OTU variance in response to warming. AM OTU variance of warm-control plots in $O$. vauvilliersii were significantly different from the other three treatments. C. rubra did not show any significant differences in AM OTU variance between removal treatments. In $V$. venustula, there was no significant difference between AM OTU variance of the ambientcontrol and warm-removal plots suggesting a confounding effect between treatments. 


\section{Discussion}

Here, I analysed how the structure and diversity of the mycorrhizal fungal community associated with native and an invasive plant species responded to the experimental warming and removal of a dominant and invasive plant species. My rarefaction analysis showed that manipulative warming and C. vulgaris removal reduced total MF diversity within plots, whereby, the combination of the treatments warming led to the greatest loss of MF diversity. My rarefaction analysis also confirmed that I sampled sufficiently to capture the mycorrhizal fungi (MF) diversity of the host plant species and experimental treatments at this site. Additionally, MRPP analyses also revealed that individual plot had no significant effect on mycorrhizal OTU composition at any level of NMS ordination of the sampling subsets.

At the plant guild and species levels, the effects of the experimental treatments on MF diversity and community structure were more complex. Warming strongly reduced the diversity of mycorrhizal fungi associated with native ErM plants, D. subulatum and E. alpina. Contrastingly, warming strongly increased the diversity of MF associated with invasive C. vulgaris. The removal of $C$. vulgaris had no significant effect on MF diversity in D. subulatum, but interacted with warming to restore MF diversity to near ambient control values. Warming and $C$. vulgaris removal both led to significant reductions in MF diversity in E. alpina, but these effects interacted antagonistically, neutralising the independent effects of the treatments in the combined warm removal treatment. Shannon's diversity of ErM fungi declined in response to warming treatment in both native ErM species, D. subulatum and E. alpina. Warming also significantly decreased 
ErM OTU richness of D. subulatum, whereas no significant effect was found in E. alpina. This implies that the native ErM plant had more similar mycorrhizal communities than either had to $C$. vulgaris. These results provide little evidence for the enhanced mutualism hypotheses. However, all ErM host plant species were revealed to be significantly different from one another, as demonstrated in the MRPP of the NMS analysis of ericoid ordination space. This further reinforces the conclusions of Grove et al., (2017) and Geml et al., (2015) in that plant-mycorrhizal fungal composition changes and reduces in diversity in response to warming and invasion, and also that these effects should be analysed at a host plant specific level. Furthermore, the interaction of ambient temperature and invasive species removal saw the most significant difference to the MF community composition in D. subulatum, whereas there was no detectable difference in composition between the other three treatments.

Collectively, these results imply that warming and the presence of C. vulgaris have a similar impact on the MF composition for D. subulatum. If this is the case, this would support the findings of Duell et al., (2016) which concluded increases in temperature may further exacerbate the proliferation mycorrhizal fungi associated with invaders. However, to determine if these factors are in effect, network analysis is required in order to resolve the key interactions between host plants and their MF OTUs. Only by identifying these interactions and their response to WaRM treatments, I can examine the mechanisms leading to the changes observed in the plant-fungal community due to warming. In doing so, this will provide further evidence of key plant-mycorrhizal fungi interactions that act as ecosystem engineers in response to warming and invasion, similar 
to those findings demonstrated by Deslippe et al., (2012) and Badano et al., (2007). Overall these results support my initial hypothesis that warming reduces MF diversity. However, this is only true of the native plants-MF community, as warming promoted the MF diversity of invasive C. vulgaris.

In the AMF plant species, OTU Richness of arbuscular mycorrhizal (AM) fungi declined in response to warming treatment across all hosts. Shannon's diversity also decreased in response to warming $C$. rubra, and $V$. venustula, whereas no treatment effect was detected in $O$. vauvilliersii. In addition, MRPP analysis revealed warming led to significant shifts in AMF community composition relative to the ambient control. This reduction in AMF diversity reflects the warming effects also observed in Heinemeyer et al., (2004) and Geml et al., (2015). Despite a reduction in frost induced limitations on AMF hyphae growth, as purported by Kilpeläinen et al., (2020), climate warming does not necessarily promote $\mathrm{AM}$ interactions within a naturally assembled alpine plant community. Removal of C. vulgaris, on the other hand, had significant positive effects on AMF diversity. For example, AMF OTU Richness increased in response to removal treatments across all AM host plant species and Shannon's diversity increased in $C$. rubra, and V. venustula. These results support my hypothesis that $C$. vulgaris removal would promote AMF diversity and richness, and aligns with the degraded mutualism hypotheses (Vogelsang and Bever, 2009) . Additionally, the combined effect of warming and removal treatment led to slight reductions in AMF diversity overall in comparison to both ambient treatments, suggesting that $C$. vulgaris removal does not wholly offset warming induced declines. Therefore I deduce that warming is the foremost factor 
reducing the diversity of arbuscular mycorrhizal fungi community compositions across all native host plants.

My species-level analysis of MF diversity and community structure also suggest that $C$. vulgaris and E. alpina are comparatively more generalist than other host plant species. For example, across all plant species $C$. vulgaris and E. alpina shared the highest MF OTU richness, with a median score of 35 and 33 in warm-control and ambientcontrol plots respectively. As a total of 47 ErM OTUs were detected, C. vulgaris and E. alpina formed interactions with $\sim 75 \%$ of the available partners, which suggests their roles as generalist species in the plant-mycorrhizal community, while D. subulatum interacts with comparatively few MF. A subset of species, particularly invaders, are expected to be interaction generalists in mutualist networks (Duell et al., 2016; Orlovich and Cairney, 2004). By comparison, in the AM interactions, C. rubra, V. venustula, and O. vauvilliersii each formed interactions with $\sim 50 \%$ of the available AMF OTUs. In light of the NMS analysis of AMF, this suggests there is less total functional overlap in the MF they host. These results support my hypothesis that as an invasive species, $C$. vulgaris is more generalist than many of the native species. Likewise, my findings align with those of previous studies that indicate invasive plants species tend to be generalist in their MF interactions (Le Roux et al., 2018; Dickie et al., 2010; Orlovich and Cairney, 2004), a factor that is predicted to enable their above-below ground dominance and successful invasion (Richardson et al., 2007). Additionally, C. vulgaris comparative lack of AMF OTUs to the other ErM hosts is evocative of the conclusions by Allen et al., (2020) whereby invaders exert stronger negative effects and exclude non-beneficial mutualists. 
The high relative interaction generality of E. alpina was somewhat unexpected, particularly compared with its historically dominant native counterpart, D. subulatum (Smale, 1990). However, these results suggest that E. alpina is non-selective and opportunistic in its interactions with MF. This finding fits well with explaining its consistent levels of OTU richness under removal treatments. Furthermore, the significant difference in MF community structure in E. alpina between the ambient-removal and warming-removal treatment suggest that warming enables E. alpina to engage in novel interactions, suggesting its functional plasticity as response to warming. To confirm this as a functional response of E. alpina, network analysis is required to resolve the plasticity and opportunism in its interactions with MF (Sheykhali et al., 2020).

In summary, my analyses supported my hypothesis that warming reduces the diversity in the composition of mycorrhizal fungi associated with native plants. In addition, my analyses also revealed that warming promoted the diversity in the composition of ericoid mycorrhizal fungi hosted by invasive C. vulgaris. While I found evidence that the removal of $C$. vulgaris promoted diversity of mycorrhizal fungi associated with host plants that form arbuscular mycorrhizas, I cannot conclude this was due to a relief in competition from the ErM network, particularly as the removal of invasive C. vulgaris increased the diversity of MF hosted by both native ericaceous species, D. subulatum and E. alpina under warming. I conclude that to wholly disentangle the independent and interactive effects of warming and the presence of an invasive species on the plant-mycorrhizal community, I must identify the response of critical plant-mycorrhizal interactions to the WaRM treatments using network analyses. 


\title{
Chapter III.
}

\section{The effects of warming and dominant species removal on plant-mycorrhizal fungal interaction networks in an alpine tussock grassland.}

\author{
Introduction
}

Anthropogenic climate change effects and species invasions independently and interactively alter the compositions of plant and below-ground communities and their functions (Duell et al., 2016; Geml et al., 2015, Grove et al., 2017). Plant-mycorrhizal fungi symbioses are ubiquitous interactions, foundational to the function of plant communities (Ferlian et al., 2018). Moreover, plant-mycorrhizal interactions have shown to be sensitive to temperature shifts and plant invasions, especially in alpine regions (Wahl and Spiegelberger, 2016).

While analyses of the richness and diversity of mycorrhizal fungi in plant roots can illuminate how these communities respond to warming and invasive plant species, these approaches are insensitive to changes in the strength of mycorrhizal interactions and do not consider the sharing of interaction partners (Van Der Heijden and Horton, 2009). Notably, interaction strengths have proven as a fundamental component of invasion success in plant-mutualist communities (Allen et al., 2020) and common interaction partners have proven foundational to the stability of mycorrhizal communities (Muneer et al., 2020). Furthermore, invasive plants have been shown to strengthen the fitness of host plants within their common mycorrhizal networks (Awaydul et al., 2019). Therefore, these essential mechanisms require the more discerning network analysis 
approach to understand how drivers of change are altering the compositions of plantmycorrhizal communities.

The advantage of a network approach is that it can decipher how species and their interactions independently and cumulatively contribute to the function of a community (Dormann et al., 2017). Most ecological community network analyses are confined to measuring interactions between two trophic levels. This type of network, which consist of two-layers, is called a "bipartite" network. Therefore, according to interaction network theory, the "function" of a species in a bipartite network is defined by the strength, diversity and topology of its interactions with the other trophic level (Dormann et al., 2009). The connectivity of a network is the sum of these properties for all species in a network. Additionally, considering species' functions relative to one another can inform those that are functionally similar or distinct, which can be useful for inferring how they may respond to alterations in community compositions.

Considering the topology of species interactions in a network can also illuminate other important functional characteristics, such as interaction plasticity, generality and specialism within the community (Dormann et al., 2017; Sheykhali et al., 2020). Determining these properties of network architecture further reveal key attributes that define a network's structure and robustness (Sheykhali et al., 2020). In particular, species that share common interaction partners, affect a redundancy that provides the network function a robustness to species loss (Biggs et al., 2020). Conversely, functional diversity among species interactions affect a greater variety in the ecosystem functions and traits present (Cadotte et al., 2011). Functionally diverse species are unlikely to experience 
identical effects in the event of species loss or disturbance, thus contributing another component of network robustness (Petchey and Gaston, 2006). High degrees of functional diversity and functional redundancy in a network contribute to "functional complementarity", reinforcing its stability (Blüthegen and Klein 2011). Functional complementarity serves as a proxy for community robustness, as it is evidence for network structural integrity that acts to preserve the interactions and species present.

The analysis of bipartite network architecture requires a methodological approach to calculate of a multitude of metrics to reflect its structure and robustness (Dormann et al., 2009). While developing network graph theory has resulted in the formulation of many metrics, Dormann et al., (2009) advises that the analysis of interaction networks be done at three distinct levels; the whole network-level, the trophic-level and the specieslevel. At each level, key aspects of network structure such as connectivity, species' functional complementarity (Petchey and Gaston, 2007) and species' centrality (Lai et al., 2012), should be analysed. In addition, computing modularity for high resolution interaction data, such as sequence reads, is considered as a powerful addition to a network analysis approach (Dormann et al., 2017). Modularity ensures sources of variance are not overlooked in favour of determining significant effects and can highlight the presences of sampling bias. This aspect is highly significant to plant-mycorrhizal network analyses as spatial sampling bias and neighbourhood effects have been revealed as highly influential on the outcome of their network analysis metrics (Bogar and Kennedy, 2013; Chagnon et al., 2020). Therefore, to test for these biases and address the 
limitations of network analysis Chagnon, (2016) insists that ecological interaction network analysis studies utilise the rigorous testing of null model networks.

In this chapter, I analyse the architecture of interactions between the mycorrhizal fungal community and a native alpine tussock grassland, in response to warming and the removal of a dominant and invasive plant species, C. vulgaris. I sample the roots of 6 plant species in each of the $32 \mathrm{WaRM}$ treatment plots and characterise the MF community as outlined in Chapter 2. Using network analyses as outlined by Dormann et al., (2009) from the bipartite package of R, I construct 32 plant mycorrhizal networks, from the fungal sequence read data on each sampled host plant. For each network, I calculate metrics pertaining to properties of network structure and robustness at the whole network, trophic-level and species/mycorrhizal fungal OTU scales. I test the following hypotheses: 1) warming will reduce the robustness and strength of mycorrhizal interactions of native plants; 2) the removal of invasive $C$. vulgaris will reduce the diversity and robustness of the ericoid mycorrhizal network and increase the diversity and robustness of the arbuscular mycorrhizal network. I then investigate the functional similarity and diversity between all samples, to determine the prevalence of neighbourhood effect, across the mycorrhizal networks of the two functional guilds (AM and ErM) by computing network modularity using the algorithm devised by Beckett (2016). Finally, I investigate which mycorrhizal fungal OTUs were influential hubs that determine a significant aspect of structure and connectivity in the plant-MF networks. 
Methods

Experimental Design and Molecular Methods

The experimental design and molecular methods used for sampling mycorrhizal fungal OTUs for network analysis are described in Chapter 2.

\section{Statistical Methods}

To visualise the interactions between host plant species and their associated mycorrhizal fungal OTUs, I constructed bipartite networks for each plot using the plotweb function in the bipartite package in R. Graph nodes, which represent plant species and fungal OTUs (hereafter, collectively "species"), were colour coded by mycorrhizal type and ordered by their overall abundance in the dataset. The width of each graph node represents the sum of its interaction frequency and strength and is proportional to the interactions realised by the other species in its trophic level. Graph node width therefore serves as a visual indication of the centrality (influence) of a species in its network. I included all plant interactions with all mycorrhizal fungi OTUs, regardless of host-plant's mycorrhizal type. For example, I included AMF that were found to occur on species that normally form ericoid mycorrhizal interactions and vice versa. Therefore I consider the response of the plant community as a whole, rather than as two independent common mycorrhizal networks (CMNs) to ensure for the highest ecological realism. In addition, I constructed mean bipartite plant-mycorrhizal networks 
for each treatment, using the mean abundance of the plant species and fungal OTUs from each of the 8 plots per treatment.

I considered the 32 bipartite plant-mycorrhizal networks at three scales; the whole network-level, trophic-level (either all host plants or all fungi), and the species-level (individual plant species or individual fungal OTUs). To preface the interpretation of the following results, it is key that I specify that all network-level, trophic-level and specieslevel metrics are weighted by the species abundance and richness in the plots.

I used the networklevel function which computes network level metrics to assess changes in total community connectivity in response to the four experimental treatments. This function outputs 19 network metrics of which I elected to present six in this thesis. I selected network metrics that describe significant aspects of overall network connectivity and structure. A full description of the network level metrics I characterised, including their calculation, source reference and interpretation in the context of my study appear in

\section{Table 13.}

For each network level metric, I assessed normality using a Levene's test in the car package of R. All metrics met this assumption of 2-way analysis of variance (ANOVA) without the need for data transformation. Therefore, I used 2-way ANOVA to assess treatment effects on the six metrics using the aov function in R. I used the ggplot 2 package of $\mathrm{R}$ to visualise how different network-level metrics responded to the four experimental treatments in a series of 6 box plots. 


\section{Table 13. Description, calculation and interpretation of the network level metrics calculated for each plot $(\mathbf{n}=\mathbf{3 2})$.}

\begin{tabular}{ll} 
METRIC & DEFINITION \\
\hline & \\
& $\begin{array}{l}\text { Totals-weighted diversity of } \\
\text { LINKAGE } \\
\text { DENSITY }\end{array}$ \\
& measure of connectivity.
\end{tabular}

\section{CALCULATION}

The totals-weighted diversity of between species (S) representing the average level of diverse (Dormann et al., 2009) connections between trophic levels.

\section{INTERPRETATION}

Increases in linkage density indicate that on average, host plants are more connected to the MF trophic level. Decreases in linkage density indicate that host plants are proportionally more disconnected from the MF trophic level, due a proportional increase in MF discrimination by host plants. Connectivity is one proxy of network robustness.

Higher weighted connectance, is akin to linkage density indicating that on average, host plants are more connected to the MF trophic level. A value of 1 indicates all host plants present are linked to all OTUs present within the network. Connectivity is one proxy of

network robustness.

Linkage density divided by number of species in the network as a proportion of maximum connectance. (Bersier et al., 2002).

\begin{tabular}{|c|c|c|c|}
\hline $\begin{array}{l}\text { SHANNON } \\
\text { DIVERSITY }\end{array}$ & $\begin{array}{l}\text { Shannon's diversity of } \\
\text { interactions. }\end{array}$ & $\begin{array}{l}\text { Calculates total network interaction } \\
\text { diversity using the diversity }= \\
\text { "Shannon" function in } \mathrm{R}\left(\mathrm{H}^{\prime}\right) \text {. } \\
\qquad \mathrm{H}^{\prime}=\sum_{\mathrm{i}=1}^{s} p_{\mathrm{i}} \ln p_{\mathrm{i}} \\
\text { Takes proportional abundance and } \\
\text { evenness of species interaction } \\
\text { diversity. (Pielou, 1966) }\end{array}$ & $\begin{array}{l}\text { Higher Shannon diversity indicates } \\
\text { more total diversity and evenness } \\
\text { of the interactions in a network. } \\
\text { Diversity of interactions, like } \\
\text { connectedness is considered a } \\
\text { proxy of network robustness and } \\
\text { function. }\end{array}$ \\
\hline
\end{tabular}

diversity. (Pielou, 1966)

Algorithm to calculate the interaction strength of species. Triplets are three nodes connected by two (open) or all three (closed)

Average strength in which

CLUSTER CO-EFFICIENT together. Calculates the (CCO;WEIGHTED) interaction strength of species. Varies between $0-1$

$$
C_{\omega}=\frac{\text { total value of closed triplets }}{\text { total value of triplets }}=\frac{\sum_{\tau_{\Delta}} \omega}{\sum_{\tau} \omega}
$$

(Opsahl and Panzarasa 2009). Derived from clustering_tm function in tnet package in $\mathrm{R}$.

NODF is based on "overlap and decreasing fill" to detect sub-set assemblages in a community. Quantitative and weighted index for nestedness (NODF function in the vegan package in $\mathrm{R}$ )

(Almeida-Neto et al., 2008). NODF outperfomed other metrics of nestedness (Ulrich et al., 2009)
Higher degrees of NODF suggest the presence of hub species with stronger effects on the structure of the plant-fungal community. Lower nestedness values suggest that the plant-fungal community are more randomly assembled. Within mutualistic networks higher NODF implies a higher resilience to disturbance and species losses. species that also interact with generalist species.
Calculates a mean dependence metric for each of the two trophic levels. Corrects for low number of interactions. Ranges from (-1 to 1 ) to describe direction of dependence (Rooney et al., 2006).
Negative values indicate higher dependence on the host plants trophic level. Positive values indicate a dependence of host plants on fungal OTUs. Increased dependence signifies higher levels disaggregation/fragmentation of a network. 
In addition, I analysed changes in network structure and robustness of each trophic level (mycorrhizal fungal OTUs and host plants) of the 32 networks. For this set of analyses, I used the grouplevel function in the bipartite package. The grouplevel function generates 16 metrics of which I elected to present the 6 most relevant in this thesis. I selected metrics that describe aspects of network functional diversity and redundancy.

Trophic level structure metrics measure whether species' (of one trophic level) observed interaction typologies are more discerning than would be expected from by chance, as calculated relative to a randomised matrix. For instance, discrepancy calculates the difference in nestedness between the observed network and a randomised matrix. C-score indicates the average proportion of interactions not selected by a species, indicating either specialism or lack of available interaction partners within the network. Togetherness calculates the number of shared interaction partners by species in a trophic level, and as such, is a proxy for functional redundancy. Robustness metrics measure the vulnerability of the trophic level to extinction; and include generality, extinction slope and functional complementarity. A full description of the metrics I selected including their calculations, source reference and interpretation are listed in Table 14. As above, I assessed normality of the trophic level metrics using the levenestest function in the car package of R. For the metrics that met the assumptions, I conducted a two-way ANOVA using the aov function in $\mathrm{R}$ to detect treatment effects. For each metric, I created box plots by treatment for the two trophic levels, and where appropriate, I showed results of both trophic levels on the same Y-axis to emphasise differences in their response. 


\section{Table 14. Description, calculation and interpretation of the trophic level analysis indices calculated for each plot $(\mathbf{n}=\mathbf{3 2})$.}

\begin{tabular}{|c|c|c|c|}
\hline METRIC & DEFINITION & CALCULATION & INTERPRETATION \\
\hline DISCREPANCY & $\begin{array}{l}\text { Nestedness calculated as the } \\
\text { discrepancy between a binary } \\
\text { matrix and the maximally } \\
\text { packed matrix (with the same } \\
\text { row sums). }\end{array}$ & $\begin{array}{l}\text { Binary interaction matrix is sorted } \\
\text { by marginal totals (A). Then, all 1s } \\
\text { in A are "pushed" to the left to } \\
\text { maximally compact the matrix (P). } \\
\text { Discrepancy = (A - P) / } 2 \text {. } \\
\text { (Brualdi and Sanderson, 1999) } \\
\text { Discrepancy regularly } \\
\text { outperfomed other measures of } \\
\text { trophic-level nestedness } \\
\text { (Ulrich et al., 2009) }\end{array}$ & $\begin{array}{l}\text { Higher discrepancy values } \\
\text { indicate a higher level of } \\
\text { nestedness. High values also } \\
\text { indicate the plant-mycorrhizal } \\
\text { network has a network more } \\
\text { structured around hub species } \\
\text { than expected of a randomly } \\
\text { generated network. Mutualistic } \\
\text { networks with higher nestedness } \\
\text { imply a higher resilience to } \\
\text { disturbance and species losses. }\end{array}$ \\
\hline
\end{tabular}

\begin{tabular}{lll}
\hline & & \\
& $\begin{array}{l}\text { Mean (normalised) number of } \\
\text { checkerboard combinations } \\
\text { across all species of one }\end{array}$ & $\begin{array}{l}\text { Transforms data to binary matrix. } \\
\text { Calculated using the formula: }\end{array}$ \\
MEAN & $\mathrm{C}=\Sigma \Sigma \mathrm{C}_{\mathrm{ij}} / \mathrm{P}$. \\
$\mathrm{i}<\mathrm{j}$
\end{tabular}

Higher the mean C-score indicates a greater degree of partner discrimination. This infers a higher frequency of undesirable partners for a trophic level. A C-score of 0 indicates that all members of trophic level interacted with all members of the other.

\begin{tabular}{ll}
\hline & $\begin{array}{l}\text { Similarity in the interaction } \\
\text { topologies of participants. An } \\
\text { indicator of functional } \\
\text { redundancy within a network } \\
\text { which is an important } \\
\text { component for robustness. }\end{array}$
\end{tabular}

Calculates the number of identical co-presences and co-absences for species in a trophic level (Stone and Roberts, 1992).

Higher togetherness scores (between 0-1) indicate higher number of identical interactions exhibited by different species within the same trophic level. Indicates functional redundancy a key component of network robustness

\begin{tabular}{|c|c|c|c|}
\hline GENERALITY & $\begin{array}{l}\text { An exponent of partner } \\
\text { diversity. Describes the level } \\
\text { of generalism in each trophic } \\
\text { level. }\end{array}$ & $\begin{array}{l}\text { Weighted mean effective number } \\
\text { of MF OTUs per host plant and } \\
\text { vice versa, weighted by their } \\
\text { marginal totals (Tylianakis et al., } \\
\text { 2007; Bersier et al., 2002). }\end{array}$ & $\begin{array}{l}\text { Lower generality infers less } \\
\text { connection to the corresponding } \\
\text { trophic level and a greater } \\
\text { vulnerability to disconnection } \\
\text { from the network }\end{array}$ \\
\hline $\begin{array}{l}\text { EXTINCTION } \\
\text { SLOPE }\end{array}$ & $\begin{array}{l}\text { Represents the slope of } \\
\text { vulnerability to extinction of } \\
\text { the trophic level from } \\
\text { multiple iterations of a } \\
\text { secondary extinction } \\
\text { sequence in one trophic level. }\end{array}$ & $\begin{array}{l}\text { Fits a hyperbolic function to the } \\
\text { extinction simulation calculated by } \\
\text { the second.extinct function. } \\
\text { (Dormann et al., 2009) }\end{array}$ & $\begin{array}{l}\text { Higher extinction slope values } \\
\text { indicate the trophic level is } \\
\text { more robust to extinctions } \\
\text { within the "partner" trophic } \\
\text { level. }\end{array}$ \\
\hline $\begin{array}{l}\text { FUNCTIONAL } \\
\text { COMPLEMENTARITY }\end{array}$ & $\begin{array}{l}\text { Community-level measure of } \\
\text { ecological niche } \\
\text { complementarity }\end{array}$ & $\begin{array}{l}\text { Measured as the total branch } \\
\text { length of a functional dendrogram } \\
\text { based on qualitative differences in } \\
\text { trophic level assemblages between } \\
\text { participants of the other trophic } \\
\text { level. (Petchey and Gaston, 2007) }\end{array}$ & $\begin{array}{l}\text { Functional complementarity } \\
\text { indicates total network } \\
\text { redundancy and diversity of } \\
\text { interactions within a trophic } \\
\text { level. }\end{array}$ \\
\hline
\end{tabular}


Following the results of the network and trophic level metrics for each plot, I considered the responses of individual host plants to the experimental treatments by exploring various species level metrics. For this analysis, I applied the specieslevel function in the bipartite package in R to every plant sample, $\mathrm{n}=141$.

These metrics quantified the structure of host plant networks and their influence on the wider plant-mycorrhizal network. A full description of the metrics I selected including their calculations, source reference and interpretation are listed in Table 15. I repeated the species-level analyses for all host plants in each plot. To assess whether the overall response of the plant-mycorrhizal network to experimental treatments was plant species-dependent, I ran linear mixed effects models of the species-level metrics with experimental treatments as fixed effects and plant species as random effects using the lme4 and lmertest functions in R. Subsequently, I split my data set by plant species and I assessed normality of the species-level metrics using the levenestest function in the car package of R. For the metrics that met the assumptions, I conducted a two-way ANOVA using the aov function in $\mathrm{R}$ to detect treatment effects. For each metric, I created box plots by treatment using the ggplot2 package in R. 


\title{
Table 15. Description, calculation and interpretation of the species level metrics calculated for each plot $(\mathbf{n}=\mathbf{1 4 1})$.
}

\author{
METRIC \\ DEFINITION \\ CALCULATION \\ INTERPRETATION
}

$\begin{array}{ll}\text { NORMALISED } & \begin{array}{l}\text { Number of interactions per } \\ \text { species divided total number } \\ \text { of partners. A measure of } \\ \text { connectivity per species. }\end{array}\end{array}$

Sum of links per species divided by the number of possible partners
Higher degree scores indicates a greater connectivity of the host plant species to the OTU trophic level. Higher values indicate higher network robustness of the species.

$\begin{array}{ll} & \begin{array}{l}\text { Effective number of partners } \\ \text { if each partner was equally } \\ \text { EFFECTIVE }\end{array} \\ \text { PARTNERS } & \begin{array}{l}\text { another reliable value of } \\ \text { connectivity of a species. }\end{array}\end{array}$

Effective number of partners, common. Considered as connectivity of a species.
Logbase to the power of "partner diversity" metric (also computed in the species-level function in the bipartite package) Based on analysis by Bersier et al., (2002)
Higher EP scores indicate a greater connectance of the host plant species to the OTU trophic level. Higher values indicate higher network robustness of the species.

$\begin{array}{ll} & \text { The number of realised } \\ \text { interactions in relation to the } \\ \text { PROPORTIONAL } & \text { potential number of partner } \\ \text { GENERALITY } & \text { species. A metric to represent } \\ \text { the degree of generality of } \\ \text { the host plant species. }\end{array}$

The number of realised potential number of partner the degree of generality of the host plant species.
"Effective partners" divided by total "potential partners".

Potential partners is calculated as the logbase to the power of "normalised degree" (Bersier et al., 2002)
Higher scores of generality indicate a greater diversity in the number of OTU partners. This is used as a proxy for how disconnected a host species is to the MF trophic level. This is also an indicator of species influence and centrality in the network.

\begin{tabular}{|c|c|}
\hline & Relative specialism or \\
\hline $\begin{array}{l}\text { BLÜTHEGEN'S } \\
\text { D' INDEX }\end{array}$ & $\begin{array}{l}\text { the discrimination of } \\
\text { interactions by the species } \\
\text { from iterations of random } \\
\text { selection of partners. }\end{array}$ \\
\hline
\end{tabular}
selection of partners.
Computes d', which is calculated based on the raw $\mathrm{d}$, dmin and dmax for each species called by the $d$ fun function (Blüthgen and Menzel, 2006).
Higher d' index scores indicate a higher level of discrimination of MF interactions by the host plant species.
Centrality of the host plants in regards to the fungal OTUs in the graph. Based on weighted representation in the network.
Computes the reciprocal of the sum of the length of the shortest paths. Uses

closeness_w function from thet package in $\mathrm{R}$.

(Opsahl and Panzarasa 2009)
Higher closeness scores indicate a higher degree of centrality the host plants have within the network. Determines centrality based on the strength of the nested and clustered structure around the host plants. This metric is a proxy for species centrality within the network.

$\begin{array}{ll}\text { SPECIES } & \text { Quantifies a species' } \\ \text { STRENGTH } & \text { influence on all its partners. }\end{array}$

Sum of dependent MF OTUs, (i.e., proportionally specialised) of each host plant species (Bascompte et al., 2006).
Higher levels of strength indicate a greater influence of the host plant to its linked MF OTUs. Note this doesn't show how strong the interactions are or which host plant is the most "central" or "influential" in the network. This metric therefore illuminates changes rather than "total" network effects. 
One of the criticisms of network analyses is that they are inevitably temporal and spatial subsets of the true underlying network, which may manifest in false significant effects. Like many experimental approaches, sampling bias is embedded in network metrics. To reduce the risk of type I errors (false rejection of null hypothesis), I ran null model tests for all network-level, trophic-level and species-level analyses. For these tests, I created null model bipartite webs with nullmodel function from the bipartite package in R. This is a wrapper function that pulls from bipartite package's shuffle.web, swap.web, vaznull and mgen, functions and the vegan package function of simulate and r2dtable. The output of the nullmodel function creates randomly generated matrices derived from my original data. Following the recommendations by Dormann et al., (2009), I ran each network-level, trophic-level and species-level metric analyses with 1000 different null model webs. All 1000 null model webs exhibited no significant treatment or species effects across all analyses. This confirms the detection of significant effects within my study are unlikely to be generated by artefacts of sampling.

Finally, I computed the modularity of the arbuscular and ericoid common mycorrhizal networks. Modules were defined as groups of host plants that were similar in both the topology and strength of their interactions. This highlighted which species were functionally similar within the context of the network. The composition of the modules revealed whether plant-MF networks were most structured by host plant species, the WaRM treatments, or the plot. Therefore, analysis of modularity allowed me to assess neighbourhood effects (plots) or other sampling bias on plant-MF networks. This approach is similar to the NMS analysis of Chapter 2 as it reveals which factor (species, 
treatment, plot) generated the greatest variation in plant-MF networks. However, the computation of modules visualises the largest source of variance within an interaction matrix, rather than in an infinite ordination space.

Currently, the most supported method for calculating modularity is the algorithm devised by Beckett, (2016). The bipartite package in $\mathrm{R}$ enables a convenient evocation of this algorithm using the computemodules function, (method = "Beckett"). The function gives a "Q value" between $0-1$, where 0 indicates a complete randomness of interactions patterns and 1 is a distinct and rigid structure, with no randomness detected after 1E10 iterations of the algorithm. Q values of $0.1-0.3$ are typical of ecological networks with many interactions observed. The plotmoduleweb function also creates a visual representation of the output. It is important that modularity is calculated for networks of true interactions, rather than for networks of co-occurances (Dormann et al., 2017). For this reason, I parsed the plant-MF community data into its two common mycorrhizal networks (CMNs). The strong segregation of AMF and ErM communities as reveal by NMS analysis in Chapter 2 further supports this approach.

Following Olesen et al., (2007) I determined which MF OTUs promoted network connectivity as generalists or formed specialist interactions with particular host plant species. The method by Olesen et al., (2007) determines the among-module connectivity ("c-value") and the within-module connectivity ("z-value") of a MF OTU, as weighted by their proportional abundance. The values are calculated as follows; 
C-values are calculated by $c=1-\operatorname{sum}\left((\mathrm{k} . i \mathrm{t} / \mathrm{k} . \mathrm{i})^{\wedge} 2\right)$, where "k.it" is the number of interactions an MF OTU has with host plants from other modules and "k.i" is the total number of interactions an MF OTU has.

Z-values are calculated by $\mathrm{z}=\mathrm{k}$.is - (ks.bar/SD.ks) where "k.is" is number of interactions an OTU has to host plant species in its own module, "ks.bar" is the average "k.is" of all host plant species in all modules "SD.ks" is the standard deviation of "k.is" of all host plant species in the module.

I calculated the C-value and Z-value, using the czvalues function in bipartite package of R, for each MF OTU from the modularity analyses of its corresponding common mycorrhizal network (arbuscular or ericoid). I then used the plot function in $\mathrm{R}$ to plot MF OTUs according to their c-values on the $\mathrm{x}$-axis, and their z-values on the $\mathrm{y}$ axis. To be considered a hub species, a MF OTU has a c-value $>0.6$ Olesen et al., (2007), and a z-value $>1.70$, Dormann and Strauss, (2014). Identifying the MF OTUs that are key interaction partners in the network and their relationship to host plants, provided insight to the species-level analyses and how treatments may impact overall connectivity and structure within the network. 
Results

Bipartite Networks

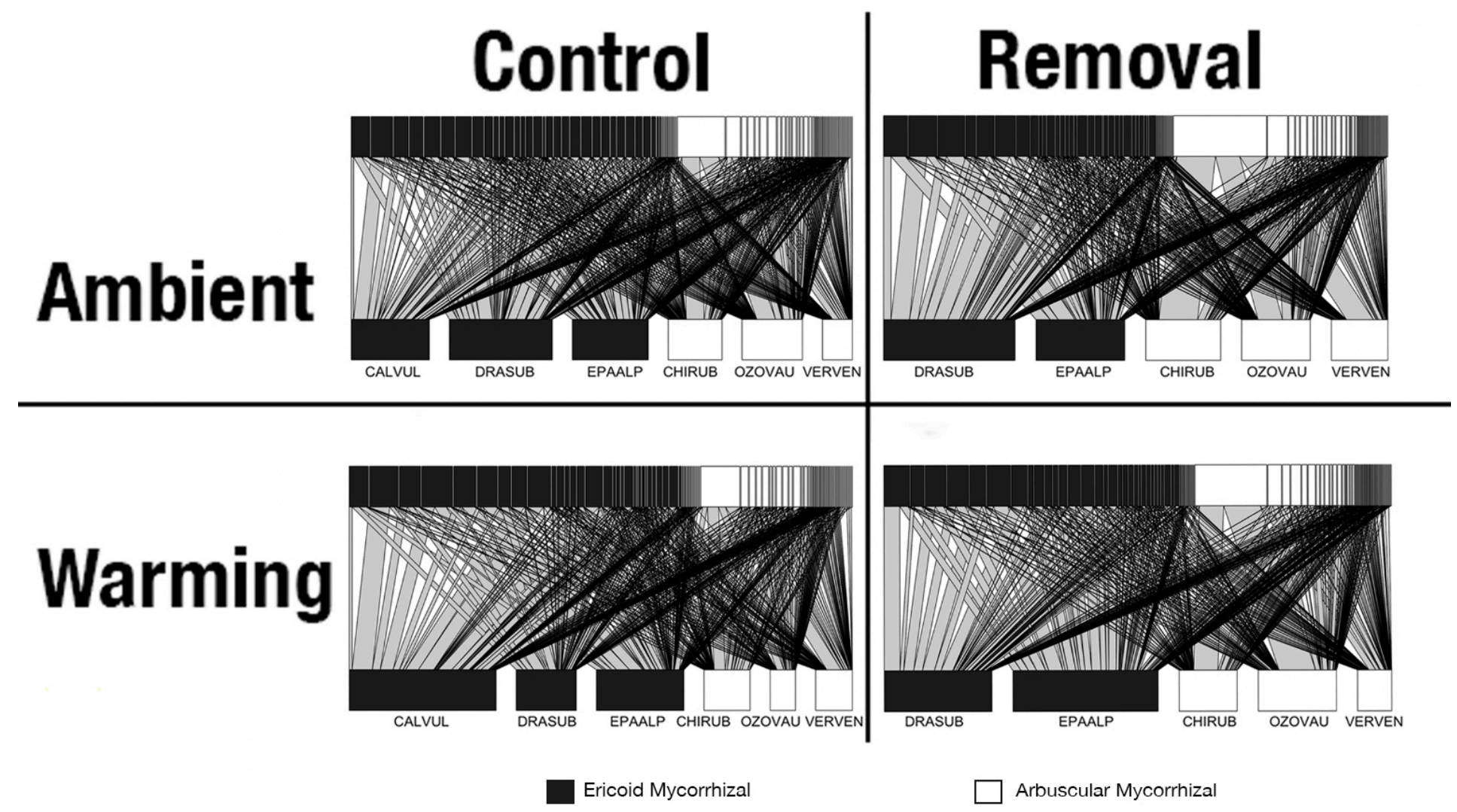

Figure 26. Mean bipartite plant-mycorrhizal networks by treatment $(\mathbf{n}=\mathbf{8})$ constructed with the plotweb function from the bipartite package in R. Host plants are on the "lower level" (lower trophic rank), connected to the ErM and AM fungal OTUs in the "higher level" (higher trophic rank). The width of the bar (i.e., graph node) for each network participant is proportional to the relative strength and diversity of its interactions, and acts as a proxy for the relative centrality (influence) of the participant on the network. The width of each link (interaction) is proportional to the relative strength of the interaction, quantified as the number of fungal OTU sequence reads that link the fungal OTU and plant species. Fungal OTUs are ordered from left to right firstly by ErM and AM fungi, and then in order of their overall rank abundance across all samples. CALVUL, Calluna vulgaris. DRASUB, Dracophyllum subulatum. EPAALP, Epacris alpina. CHIRUB, Chionochloa rubra. OZOVAU, Ozothamnus vauvilliersii. VERVEN, Veronica venustula. Bipartite networks for each plot can be found in Appendix 2, Figure 1-4. 
Experimental warming and dominant plant species removal treatments led to strong and significant changes in the architecture of plant-mycorrhizal networks in this alpine tussock grassland (Figure 26). Foremost of these effects was warming treatment, which increased the relative abundance and strength of interactions realised by $C$. vulgaris and E. alpina within the network, indicating an increase in their centrality to the network. Conversely, warming appeared to decrease the centrality of D. subulatum and all AM host plant species in the network. As expected, removal of invasive C. vulgaris in the dominant species removal treatment increased the relative abundance and strength of interactions enjoyed by all other plant species. However, the warm-removal treatment (where C. vulgaris was absent) promoted the centrality of both E. alpina and $O$. vauvilliersii in the network. This suggests that the mycorrhizal interactions that were most strongly associated with $D$. subulatum, C. rubra and $V$. venustula may be relatively less tolerant to warming than those of E. alpina and $O$. vauvilliersii, irrespective of $C$. vulgaris removal.

The two most abundant AM OTUs, OTU686 and OTU1054, both appeared to exhibit high degrees of generality with all AM hosts, regardless of the strength of their interactions or treatment. However, the most abundant ErM OTUs appeared to exhibit higher levels of interaction partner discrimination. OTU773, the most abundant ErM OTU, had strong interactions with D. subulatum and E. alpina and comparatively weaker interactions with $C$. vulgaris. Whereas the second most abundant ErM (OTU739), exhibited a high level of specialist interaction C. vulgaris, that increased in proportional centrality in the warming treatment. In the absence of $C$. vulgaris in the ambient-removal 
treatment, OTU739 maintained its proportional centrality in the network by switching partners to form strong interactions with D. subulatum and E. alpina. However, in the warm-removal treatment, the centrality of OTU739 decreased dramatically, particularly in its reduction of interaction strength with $D$. subulatum. This suggests that warming may have augmented the effect of the removal treatment, possibly by increasing the rate of rhizosphere processes and allowing for greater architectural change in the plantmycorrhizal network. However, this result could also indicate D. subulatum increased in the proportional strength of its interactions with other OTUs, becoming relatively more connected to the OTU trophic level than in the ambient-removal plots. 
Analysis of Network Level indices
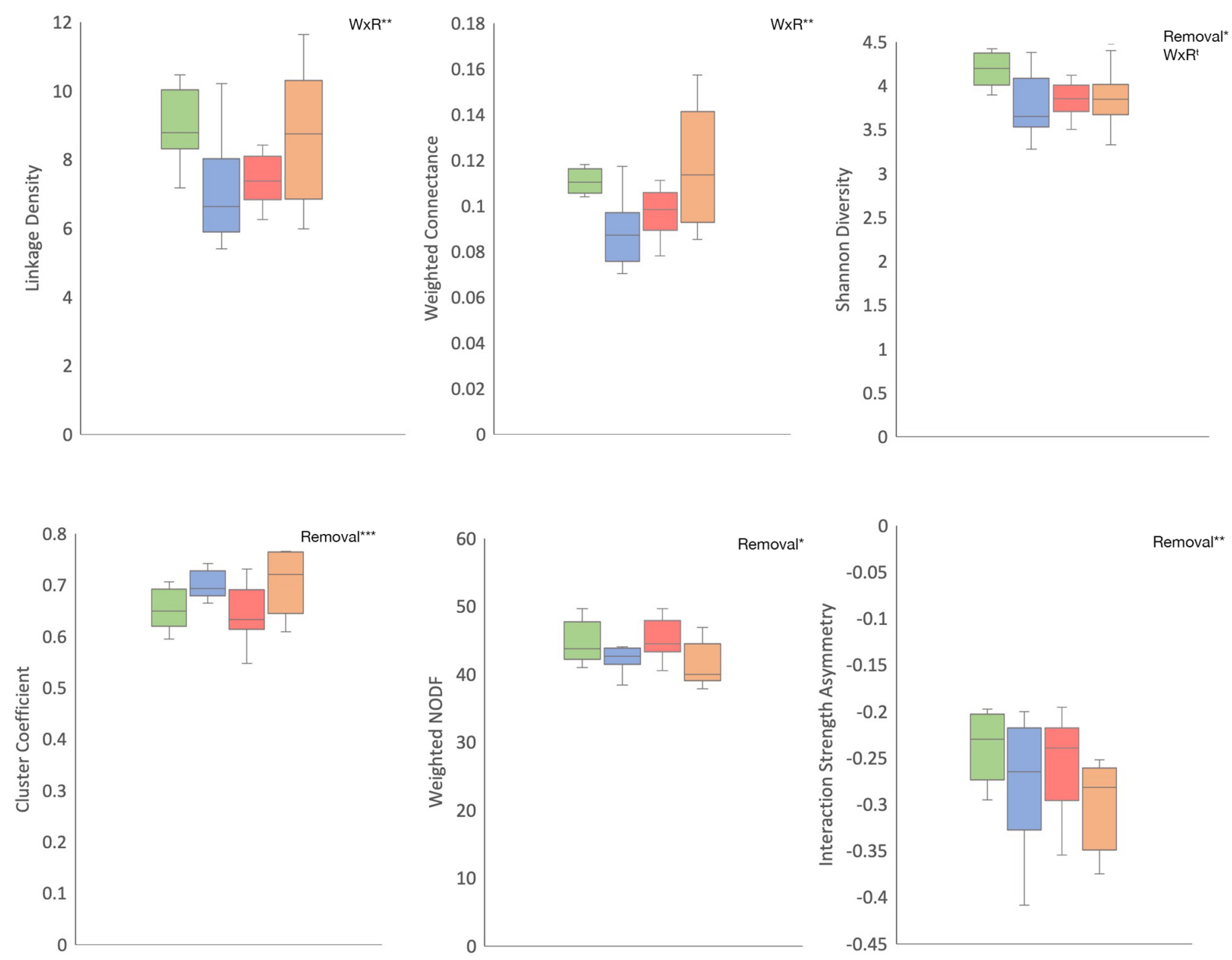

$\square$ ambient-control $\square$ ambient-removal $\square$ warm-control $\square$ warm-removal

Figure 27. Network level metrics for plant-mycorrhizal networks in response to factorial warming and removal treatment ( $n=8$ per treatment, 32 total). Median values for each metric are denoted by a horizontal line, boxes represent the interquartile ranges and whiskers denote the upper and lower 95\% confidence intervals for each metric in response to the four treatments. All metrics were calculated with the networklevel function in the bipartite package in $\mathrm{R}$. The analyses are organised by rows of analyses pertaining to network structure and network interactions. Significant treatment effects from the Two-Way ANOVA are denoted as; *** $\mathrm{p}<0.0005, * * \mathrm{p}<0.005, * \mathrm{p}<0.05$. 
The analysis of network-level metrics (Figure 27) provided further insight as to how the architecture of the plant-mycorrhizal network shifted in response to the factorial combination of warming and dominant species removal treatment, after 4 years.

Linkage density, which reflects the average diversity of interactions by host plant species and mycorrhiza fungal OTUs, was reduced with $C$. vulgaris removal and warming treatment independently, but the combination of treatments offset their individual effects. This led to a significant interaction of warming and dominant species removal treatments on linkage density. Similarly, weighted connectance, which is the proportion of realised interactions among all possible interactions, was reduced with $C$. vulgaris removal and warming treatment, but recovered in the combined treatment leading to a significant interaction of the treatment effects. These results indicate host plants were on average less connected to the MF trophic level under the ambient-removal and warm-control treatments, and the interaction between the two treatments significantly determines the connectivity of the network. However, Shannon diversity of interactions significantly decreased with removal treatment and interacted with warming, so that warming offset the removal effect. These results support the view that in the ambientcontrol treatment the plant-MF networks are significantly more connected and have a greater diversity of interactions than in the other three treatments. This also indicates that while warm-removal plots recovered network connectivity, there was not a recovery in diversity of interactions, suggesting a loss in total diversity of MF available to host plants. 
The cluster coefficient, which measures the strength of interactions of host plant species to their associated MF, significantly increased with removal and exhibited no warming effect. Therefore, C. vulgaris removal increased the strength of interactions between the native plants and their MF networks. In contrast, removal treatment significantly reduced the nestedness (weighted NODF) of the network. This implies that C. vulgaris is a significant hub species, that promotes network connectivity and the accrual of specialist and rare MF. Likewise, this result may suggest that $C$. vulgaris is more generalist or interacts more frequently with generalist MF than the native host plants. Additionally, C. vulgaris removal saw significant declines in the interaction strength asymmetry value. This negative values signify that MF abundance and interactions are dependent upon the host plant assemblage, particularly C. vulgaris.

Collectively, the network-level results indicate that invasive C. vulgaris is an influential generalist that acts to increase connectivity of MF to the host plant trophic level. However, when $C$. vulgaris is removed, there was a strengthening of the interactions between native plants and their fungal OTUs. This indicates that the presence of $C$. vulgaris suppresses the average strength of interactions of host plants and their MF. Although warming did not have an independent effect on the network-level metrics, it reduced the connectivity of the network under the control treatments. Furthermore, as warming did not offset the removal effect of MF dependency, this indicates that the network of warm-removal treatment is more fragmented than that of the ambient-control treatment, despite similar levels of average host plant connectivity. 
Analysis of Trophic Level Structure

MF OTUs
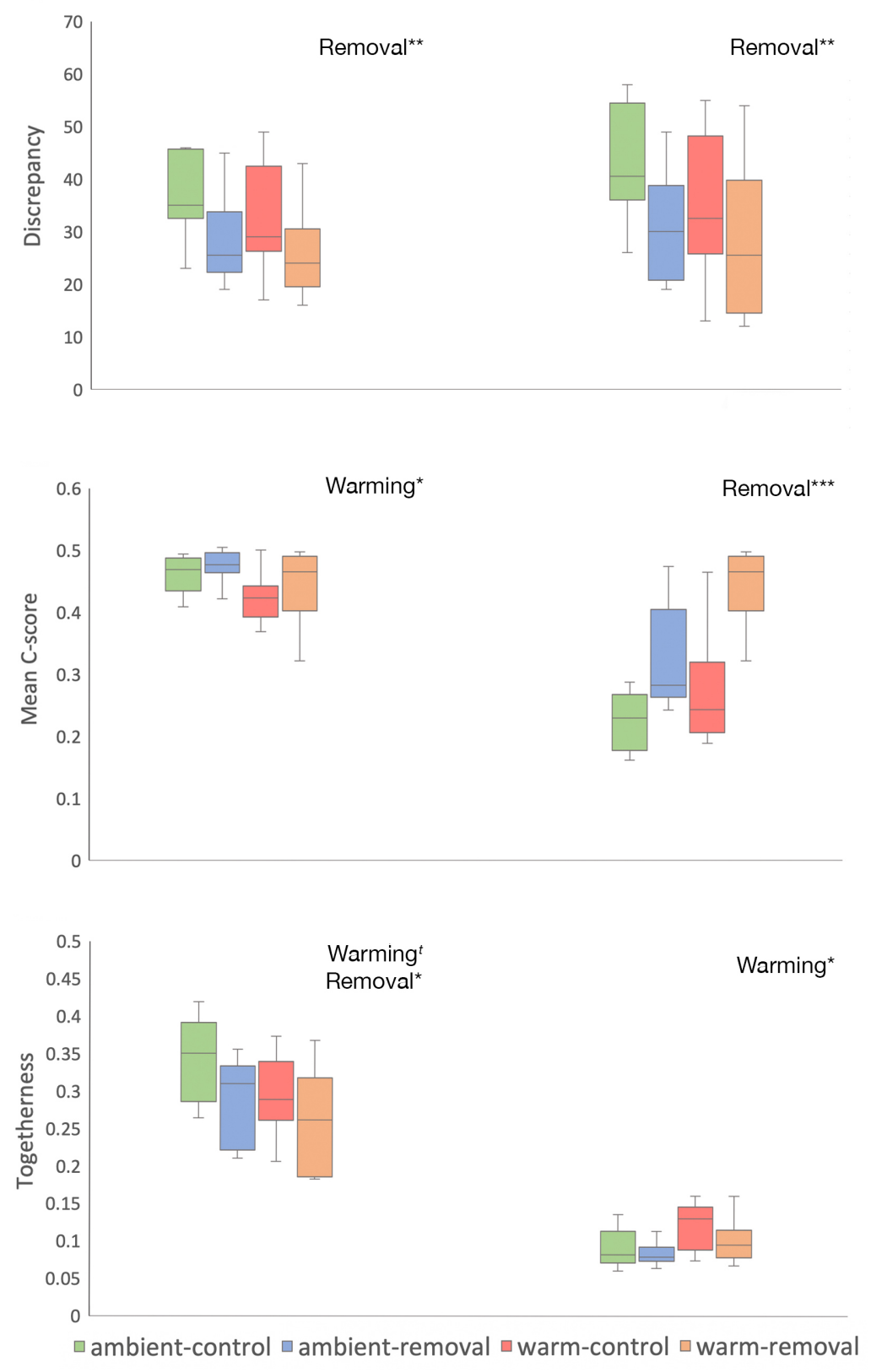

Host Plants

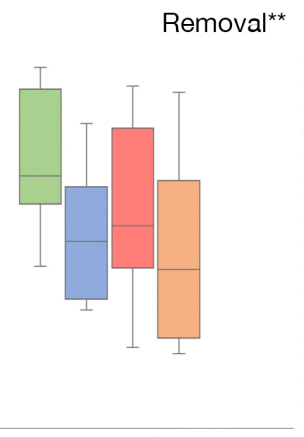

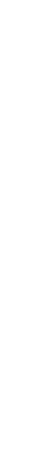

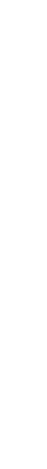

Figure 28. Trophic-level "structure" metrics for plant-mycorrhizal networks in response to factorial warming and removal treatments $(n=8$ per treatment, 32 total $)$. Median values for each metric are denoted by a horizontal line, boxes represent the interquartile ranges and whiskers denote the upper and lower 95\% confidence intervals for each metric in response to the four treatments. All metrics were calculated with the grouplevel function in the bipartite package in R. Significant treatment effects from the Two-Way ANOVA are noted with $* * *$ if $\mathrm{p}<0.0005, * *$ if $\mathrm{p}<0.005, *$ if $\mathrm{p}<0.05$. WxR, Warming and Removal factors as a significant treatment effect. 
As foreshadowed in the weighted NODF analyses, removal treatment significantly reduced the nestedness (discrepancy) value at each trophic level, this confirms $C$. vulgaris is as an influential hub species that maintains a significant degree of the specialist and rare interactions within the network at both trophic levels. In contrast, CScore, which reflects the degree of partner discrimination, increased in host plants due to C. vulgaris removal. This suggests on average the native host plants are comparatively less generalist than C. vulgaris. Additionally, C-Score decreases significantly due to warming at the OTU trophic level. This implies a loss of functional differentiation between OTUs, which this may be due either to a loss diversity at the MF trophic level, or the unlikely circumstance of MF increasing in their generality. However, togetherness decreased significantly due to warming treatment and removal treatment, which indicates that functional redundancy declined with these treatments. Taken together, these results (Figure 28) suggest that warming leads to a loss of diversity and specialist interactions in the OTU trophic level. Within host plants, warming increases the functional redundancy, which reflects a comparative loss of diversity in the MF trophic level.

In summary, these results suggest that nestedness structure of the network is significantly increased by the $C$. vulgaris. It also provides further evidence that $C$. vulgaris is a generalist and significantly more so than the native host plants. Additionally, these results highlight that warming reduces both functionally diverse and redundant interactions, lowering the robustness and diversity within the network. 
Analysis of Trophic Level Robustness

MF OTUs
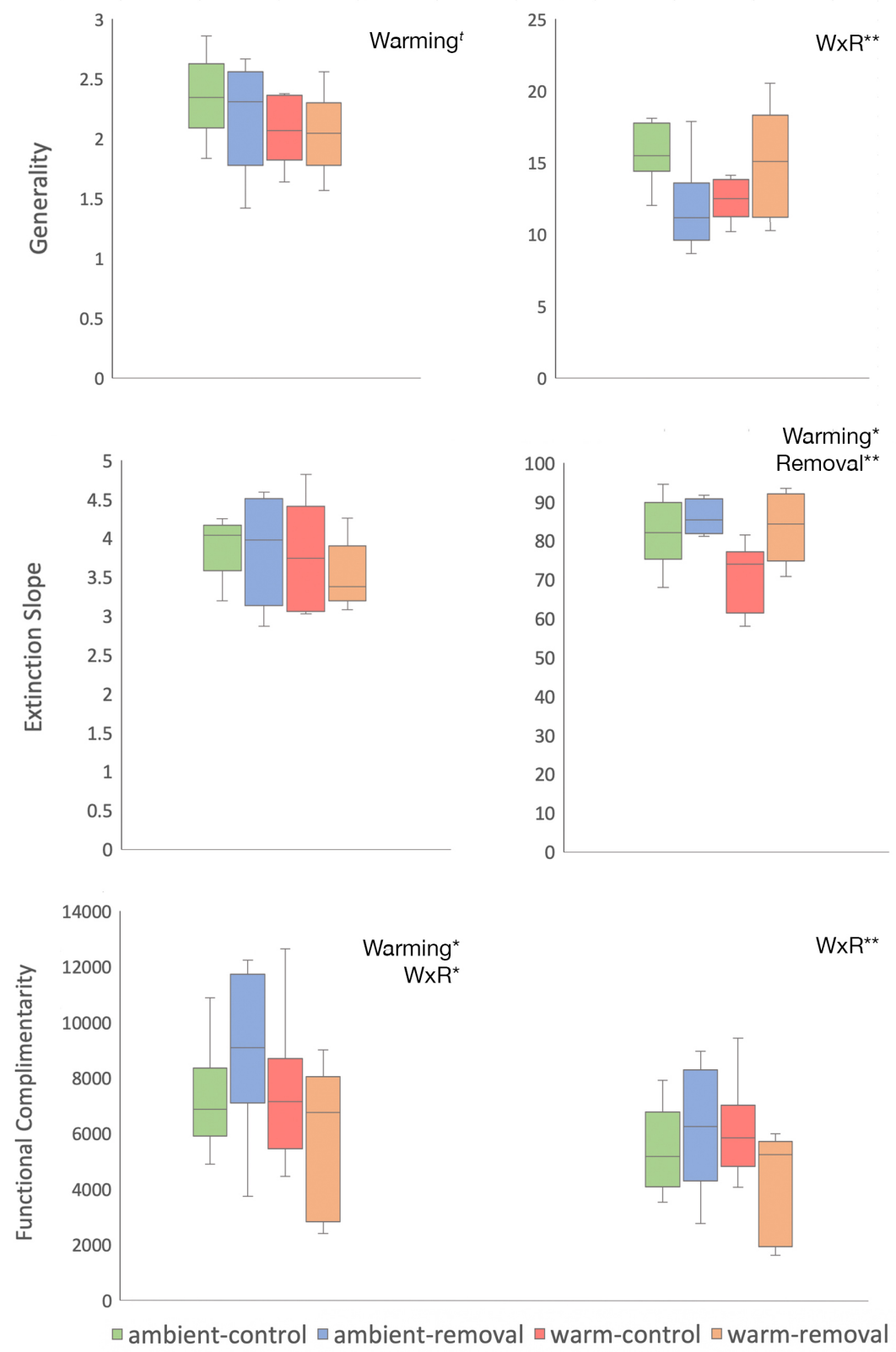

Host Plants
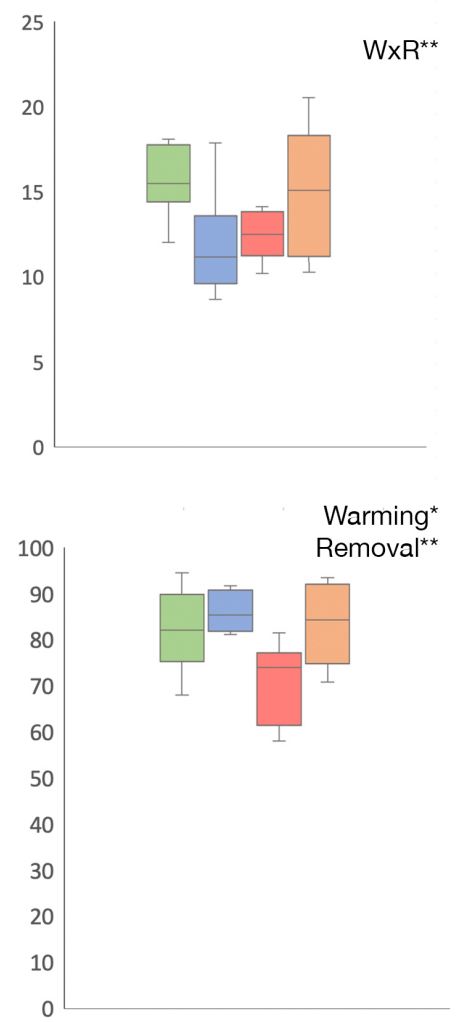

$\mathrm{W} \times \mathrm{R}^{\star *}$

Figure 29. Trophic-level "robustness" metrics for plant-mycorrhizal networks in response to factorial warming and removal treatments $(n=8$ per treatment, 32 total). Median values for each metric are denoted by a horizontal line, boxes represent the interquartile ranges and whiskers denote the upper and lower 95\% confidence intervals for each metric in response to the four treatments. All metrics were calculated with the grouplevel function in the bipartite package in R. Significant treatment effects from the Two-Way ANOVA are noted with $* * *$ if $\mathrm{p}<0.0005, * *$ if $\mathrm{p}<0.005, *$ if $\mathrm{p}<0.05$. WxR, Warming and Removal factors as a significant treatment effect. 
Warming significantly decreased the generality of OTUs. This confirms a loss of functional redundancy within the network, which agrees with my findings for togetherness. Overall, the generality indices of host plants reflected the connectivity metrics at the network level, as all three metrics reflect the average diversity of interactions for each plant species. As there are far fewer interactions possible for MF OTUs than host plants, changes in the MF connectivity were masked at the network level. WaRM treatments had no significant effect on the extinction slope value at the MF trophic level. Therefore, treatment did not impact the likelihood of extinction of MF when host plants were eliminated at random. This suggests there are relatively few specialist MF. However, warming significantly increased the extinction likelihood of host plants, while removal treatment offset the warming effect. This result supports the evidence that interaction strengths of other host plants are suppressed by C. vulgaris and that warming decreases connectivity of host plants to the MF trophic level. Similarly, functional complementarity, which indicates the cumulative degree of network functional diversity and redundancy, decreases at the MF trophic level due to warming. Removal treatment also increases functional complementarity at the MF and host plant trophic levels, however when combined with warming, the removal effect is reversed.

Collectively, these results (Figure 29) confirm that warming significantly reduces network robustness. C. vulgaris removal only offset the warming impact in the unlikely event of MF extinction eliminating the host plant trophic level. Conversely, C. vulgaris removal significantly increased functional complementarity, however warming, reversed this effect, suggesting C. vulgaris as integral to network connectivity under warming. 
Analysis of Species Level Structure
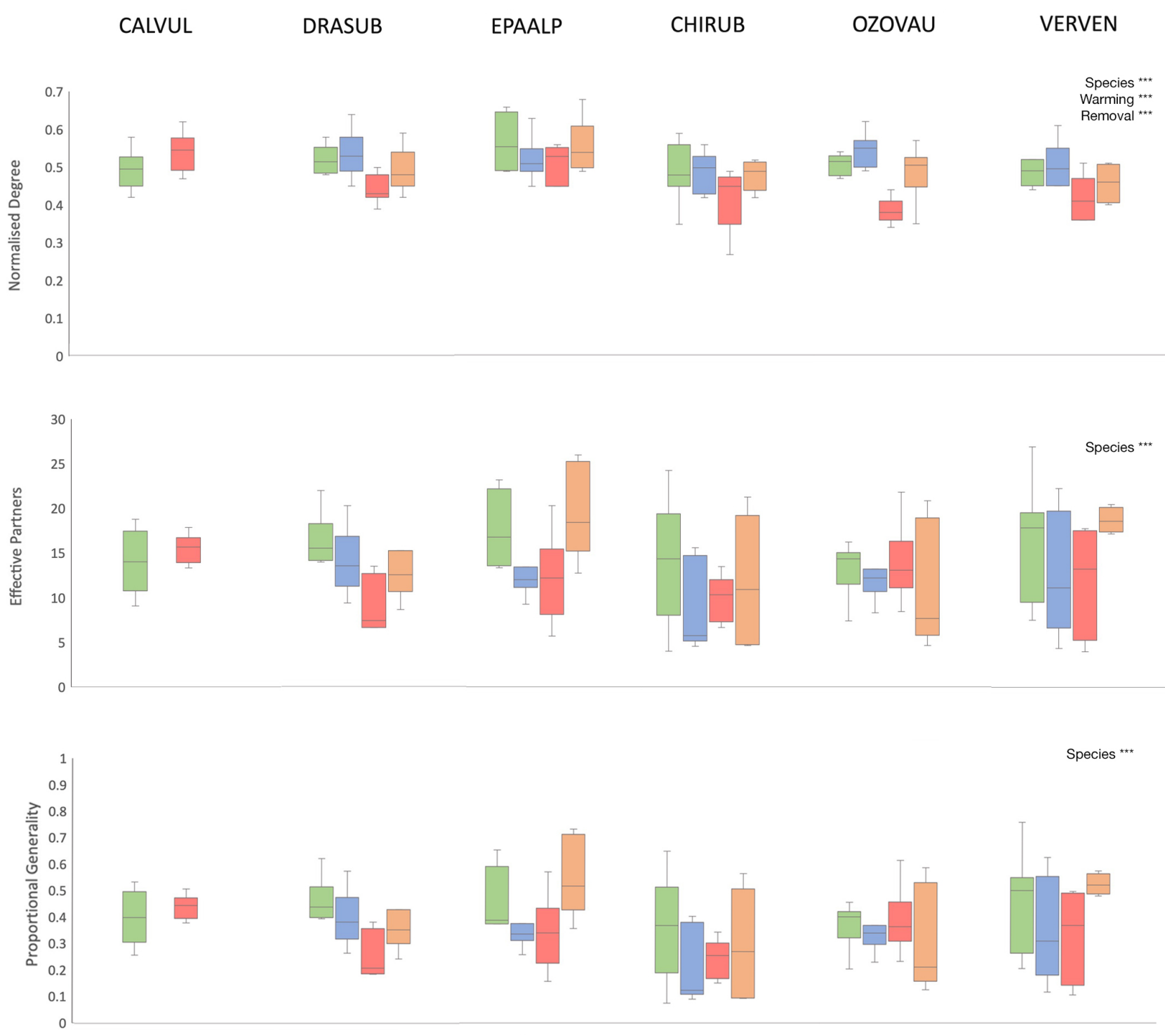

$\square$ ambient-control $\square$ ambient-removal $\square$ warm-control $\square$ warm-removal

Figure 30. Species-level "structure" metrics for plant-mycorrhizal networks in response to factorial warming and removal treatments $(n=4-8$ per treatment, 32 total). Median values for each metric are denoted by a horizontal line, boxes represent the interquartile ranges and whiskers denote the upper and lower $95 \%$ confidence intervals for each metric in response to the four treatments. All metrics were calculated with the specieslevel function in the bipartite package in R. Significant treatment effects from the Two-Way ANOVA are noted with $* * *$ if $\mathrm{p}<0.0005, * *$ if $\mathrm{p}<0.005, *$ if $\mathrm{p}<0.05$. WxR, Warming and Removal factors as a significant treatment effect. CALVUL, Calluna vulgaris. DRASUB, Dracophyllum subulatum. EPAALP, Epacris alpina. CHIRUB, Chionochloa rubra. OZOVAU, Ozothamnus vauvilliersii. VERVEN, Veronica venustula. 
Table 16. Two-Way ANOVA results of species-level "structure" metrics by host-plant species. CALVUL, Calluna vulgaris. DRASUB, Dracophyllum subulatum. EPAALP, Epacris alpina. CHIRUB, Chionochloa rubra. OZOVAU, Ozothamnus vauvilliersii. VERVEN, Veronica venustula. Significant treatment effects from the Two-Way ANOVA are denoted $* * *$ if $\mathrm{p}<0.0005, * *$ if $\mathrm{p}<0.005, *$ if $\mathrm{p}<0.05,{ }^{t}$ if $\mathrm{p}<0.10$ with "+" for increase and "-" for decrease effect. Full results of the Two-Way ANOVA are listed in Appendix 1. Supplementary Table 7.

\begin{tabular}{|c|c|c|c|c|}
\hline INDEX & SPECIES & WARMING & REMOVAL & WXR \\
\hline NORMALISED & CALVUL & $+* *$ & N/A & N/A \\
\hline \multirow[t]{5}{*}{ DEGREE } & DRASUB & $-*$ & $+t$ & 0 \\
\hline & EPAALP & 0 & 0 & 0 \\
\hline & CHIRUB & 0 & 0 & 0 \\
\hline & OZOVAU & $-* * *$ & $+*$ & 0 \\
\hline & VERVEN & $-* *$ & 0 & 0 \\
\hline EFFECTIVE & CALVUL & 0 & N/A & N/A \\
\hline \multirow[t]{5}{*}{ PARTNERS } & DRASUB & $-* *$ & 0 & * \\
\hline & EPAALP & 0 & 0 & $* *$ \\
\hline & CHIRUB & 0 & 0 & 0 \\
\hline & OZOVAU & 0 & 0 & 0 \\
\hline & VERVEN & 0 & 0 & $*$ \\
\hline PROPORTIONAL & CALVUL & 0 & N/A & N/A \\
\hline \multirow[t]{5}{*}{ GENERALITY } & DRASUB & $-* *$ & 0 & * \\
\hline & EPAALP & 0 & 0 & $* *$ \\
\hline & CHIRUB & 0 & 0 & 0 \\
\hline & OZOVAU & 0 & 0 & 0 \\
\hline & VERVEN & 0 & 0 & * \\
\hline
\end{tabular}

Within the linear model across all species, warming treatment significantly decreased the normalised degree, which is the number of interactions by a host plant. Removal treatment however, significantly increased number of interactions of a host plant. No other independent treatment effects were detected with the other structure 
metrics of "effective partners" and "proportional generality" In addition, the response to treatment effects in all structure metrics were dependent on the host plant species.

At the species level, warming significantly increased the number of interactions by of $C$. vulgaris, and significantly decreased that in D. subulatum, O. vauvilliersii and $V$. venustula, as evidenced in the normalised degree metric. However, C. vulgaris removal significantly increased normalised degree in D. subulatum, and O. vauvilliersii. Conversely, warming treatment significantly decreased the diversity of interactions of $D$. subulatum, as evidenced in the declines of effective partners and proportional generality metrics. The warming-induced declines observed in the effective partners and proportional generality of $D$. subulatum and $V$. venustula, had a significant interaction with removal treatment, whereby $C$. vulgaris removal offset the warming effect, although to lesser extent in D. subulatum. In contrast, C. vulgaris removal decreased the effective partners and proportional generality in E. alpina, although warming treatment significantly offset this decline as observed in the warm-removal plots.

In summary, warming reduces the connectivity of D. subulatum, V. venustula, and O. vauvilliersii to the MF trophic level (Figure 30, Table 16). In addition, warming also reduced the diversity of interactions by $D$. subulatum and $V$. venustula, which was then partially offset by the removal treatment. Warming increased the number of interactions by $C$. vulgaris but did not significantly increased the diversity of its interactions. Finally, E. alpina shows a reduction in connectivity and diversity of interactions due to removal treatment, which is then offset by warming treatment. C. rubra saw no treatment effect on the structure of its interactions. 
Analysis of Species Level Influence

$\begin{array}{lllll}\text { CALVUL DRASUB } & \text { CHIRUB } & \text { OZOVAU } & \text { VERVEN }\end{array}$
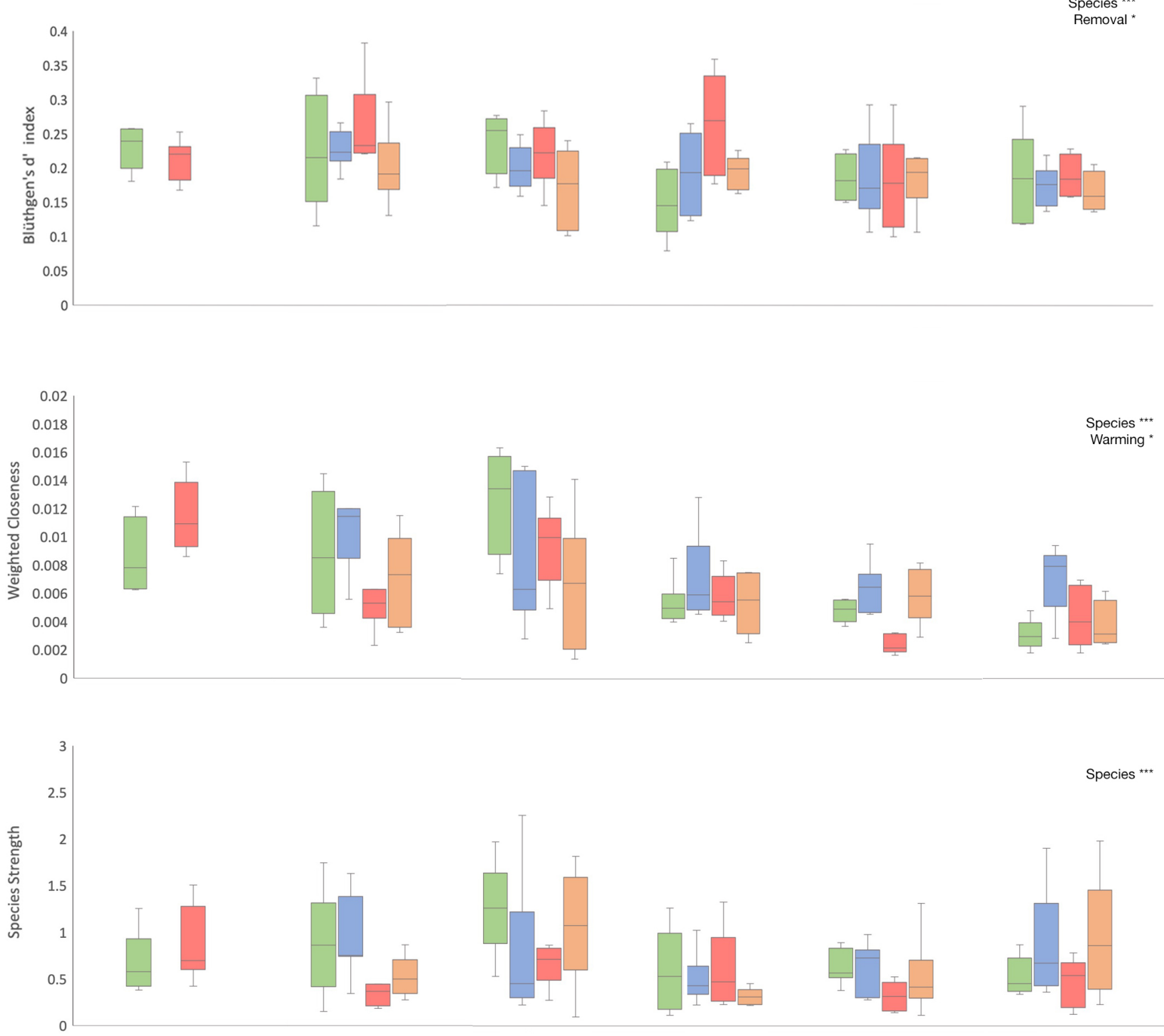

$\square$ ambient-control $\square$ ambient-removal $\square$ warm-control $\square$ warm-removal

Figure 31. Species-level "robustness" metrics for plant-mycorrhizal networks in response to factorial warming and removal treatments $(n=4-8$ per treatment, 32 total). Median values for each metric are denoted by a horizontal line, boxes represent the interquartile ranges and whiskers denote the upper and lower $95 \%$ confidence intervals for each metric in response to the four treatments. All metrics were calculated with the specieslevel function in the bipartite package in R. Significant treatment effects from the Two-Way ANOVA are noted with $* * *$ if $\mathrm{p}<0.0005, * *$ if $\mathrm{p}<0.005, *$ if $\mathrm{p}<0.05$. WxR, Warming and Removal factors as a significant treatment effect. CALVUL, Calluna vulgaris. DRASUB, Dracophyllum subulatum. EPAALP, Epacris alpina. CHIRUB, Chionochloa rubra. OZOVAU, Ozothamnus vauvilliersii. VERVEN, Veronica venustula. 
Table 17. Two-Way ANOVA results of species-level "robustness" metrics by host-plant species. CALVUL, Calluna vulgaris. DRASUB, Dracophyllum subulatum. EPAALP, Epacris alpina. CHIRUB, Chionochloa rubra. OZOVAU, Ozothamnus vauvilliersii. VERVEN, Veronica venustula. Significant treatment effects from the Two-Way ANOVA are denoted ${ }^{* * *}$ if $\mathrm{p}<0.0005, * *$ if $\mathrm{p}<0.005, *$ if $\mathrm{p}<0.05,{ }^{t}$ if $\mathrm{p}<0.10$ with "+" for increase and "-" for decrease effect. Full results of the Two-Way ANOVA are listed in Appendix 1. Supplementary Table 8.

\begin{tabular}{|c|c|c|c|c|}
\hline INDEX & SPECIES & WARMING & REMOVAL & WXR \\
\hline BLÜTHEGEN'S & CALVUL & 0 & $\mathrm{~N} / \mathrm{A}$ & N/A \\
\hline \multirow[t]{5}{*}{ D' INDEX } & DRASUB & 0 & 0 & 0 \\
\hline & EPAALP & 0 & $-*$ & 0 \\
\hline & CHIRUB & $+*$ & 0 & ** \\
\hline & OZOVAU & 0 & 0 & 0 \\
\hline & VERVEN & 0 & 0 & 0 \\
\hline WEIGHTED & CALVUL & $+* *$ & N/A & N/A \\
\hline \multirow[t]{5}{*}{ CLOSENESS } & DRASUB & $-*$ & 0 & 0 \\
\hline & EPAALP & 0 & 0 & 0 \\
\hline & CHIRUB & 0 & 0 & 0 \\
\hline & OZOVAU & $-* *$ & $+* * *$ & 0 \\
\hline & VERVEN & 0 & $+* *$ & $* * *$ \\
\hline SPECIES & CALVUL & $+*$ & N/A & N/A \\
\hline \multirow[t]{5}{*}{ STRENGTH } & DRASUB & $-* *$ & 0 & 0 \\
\hline & EPAALP & 0 & 0 & 0 \\
\hline & CHIRUB & 0 & 0 & 0 \\
\hline & OZOVAU & $-*$ & 0 & 0 \\
\hline & VERVEN & 0 & $+*$ & 0 \\
\hline
\end{tabular}

Overall plant species, removal treatment significantly decreased the Blüthegen's D' index, which is representative of MF discrimination by host plants. Warming treatment significantly also significant decreased the weighted closeness, a metric for centrality and influence within a network. The response to treatment effects in all robustness metrics were dependent on the host plant species. 
Warming treatment significantly increased the MF discrimination observed by $C$. rubra. This indicates that warming reduced the number of AMF OTUs that interact with C. rubra. However, the warming effect is offset when a removal treatment is applied. Warming treatment also significantly decreased MF discrimination in C. vulgaris, suggesting that warming promotes a higher proportion of ErM than AMF in the fungal trophic level. In addition, removal treatment also significantly decreased the MF discrimination by E. alpina, increasing the number of ErM OTUs that interact with $E$. alpina.

Warming decreased the centrality and species strength of D. subulatum and $O$. vauvilliersii. In turn, warming significantly increases centrality of $C$. vulgaris. This is indicative of a shift in the composition of mycorrhizal fungal, whereby warming further increases the influence of $C$. vulgaris in the interaction network. Removal treatment increases the centrality of $O$. vauvilliersii and centrality and strength of $V$. venustula. Warming however offsets the removal effect in centrality of $V$. venustula, further suggest warming decreases the proportional diversity of AMF available to the native host plant species. In summary, warming reduces the network influence of $D$. subulatum and $O$. vauvilliersii, and in turn promotes the influence of C. vulgaris (Figure 31, Table 17). Warming also reduced the number of available AMF interactions for C. rubra. Removal treatment saw a recovery of $O$. vauvilliersii network influence and a net increase in $V$. venustula. Finally, removal treatment also increased the availability of MF OTUs that interact E. alpina 
Modularity

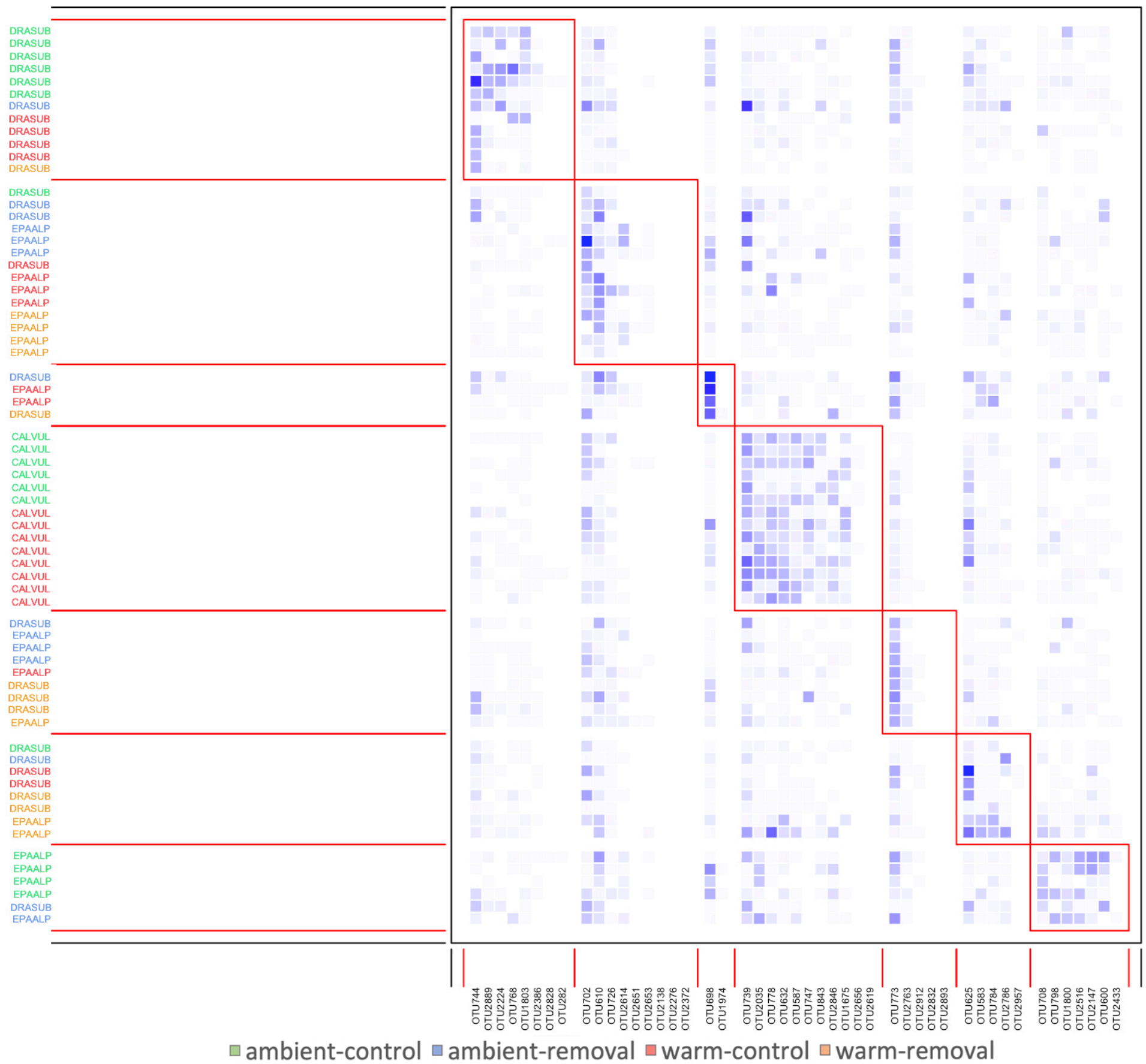

Figure 32. Interaction matrix featuring modules of the ericoid mycorrhizal network Modules were identified by LPAwb+ method (Beckett, 2016), using computemodules function in the bipartite package in $\mathrm{R}$. where $\mathrm{Q}=0 \cdot 29$. Darker squares indicate stronger interactions. Red boxes delineate the seven modules. CALVUL, Calluna vulgaris. DRASUB, Dracophyllum subulatum. EPAALP, Epacris alpina. 
The ErM network was strongly modular, scoring a Q value of 0.29 , which is high relative to many complex ecological networks (Figure 32) (Grilli et al., 2016; Dormann et al., 2017; Schleuning et al., 2014). No modules corresponded to plot or treatment. In the central module, all $C$. vulgaris samples occupy a single module, with no overlap with other host plants. Neither D. subulatum or E. alpina exhibited a similar typology of interactions to $C$. vulgaris samples. This signifies a distinct difference in network function between $C$. vulgaris and the native ErM host plant species. The C. vulgaris module also exhibits a consistent and typical "network hub" structure across treatments, with strong interactions with all the MF OTUs identified in its hub. The module is a strong indicator of $C$. vulgaris contribution to the nestedness value of the network with many strong interactions with both common and rare OTUs.

D. subulatum and E. alpina co-occur in 5/6 modules they are identified in, with the 1 st module (left to right) being the only module where E. alpina is absent. It is assumed that under D. subulatum extinction, these OTUs will lose connectivity in the network. As OTU744 was identified as the strongest proponent of this module it is also key to understand the hub status of this OTU. In addition, the $7^{\text {th }}$ module highlights ErM OTUs that significantly lost connectivity due to warming treatment, as strong interactions with D. subulatum and E. alpina were only found in the ambient treatments. There appears to be no significant removal effect on the total network structure or interaction topologies of D. subulatum and E. alpina in this network 


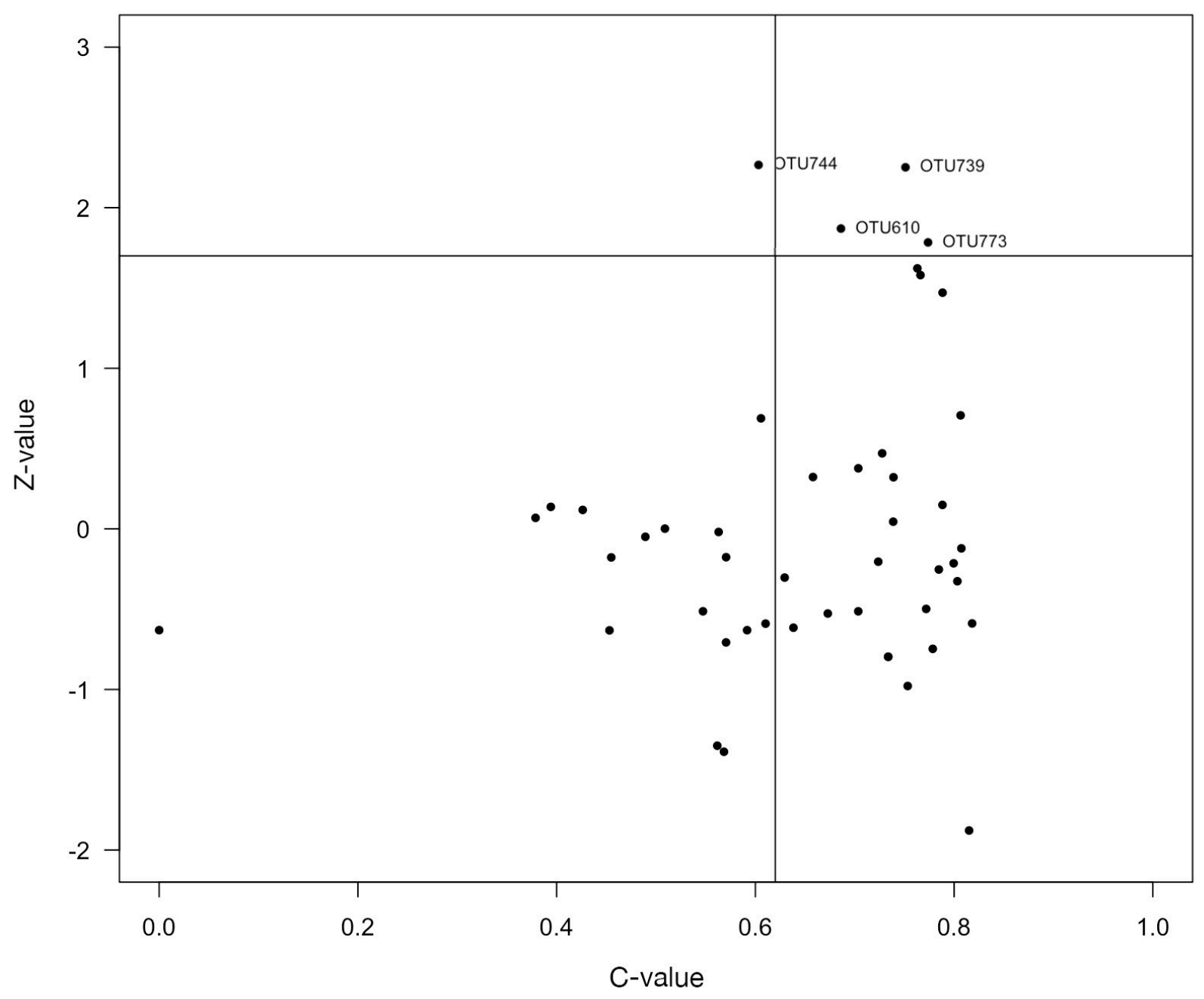

Figure 33. Connection (c) and participation (z) values plotted for ericoid mycorrhizal fungi OTUs in the network of ericaceous host plant species. Values calculated using computemodules and czvalues function in the bipartite package in R. Black lines indicate critical values according to Olesen et al., (2007) C-value (Connection) $=0.62$ and Dormann and Strauss (2014) Z-value (Participation) $=1.70$ for weighted bipartite networks. Top right quantile indicates OTUs exceeded both thresholds and are considered network "hub" species.

OTU739 exhibited the highest level of combined among-module (C-values) and within-module (Z-values) connectivity. As identified to be the most common and strongest interaction partner for $C$. vulgaris host plants, this OTU promote $C$. vulgaris network strength as exemplified with its high Z-value but is also a promoter for overall network connectivity between all modules. Thus, despite having a strong relationship with C. vulgaris, the theoretical increase of OTU739 dominance due to warming 
indicates that it will promote total ErM network connectivity, rather than singularly promoting its most common interaction partner, C. vulgaris.

OTU610 and OTU773 both share strong interactions with E. alpina and D. subulatum within two distinct modules, regardless of treatment or plot number. This suggests that while these host plants strongly interact with both OTUs, the OTUs do not significantly co-occur more than on a random basis. This indicates potential competition between the OTUs modules independent of treatment effects or spatial co-occurance. However, like OTU739, both OTUs not only promote connectivity of the modules they are within, but the connectivity of the whole network.

Conversely, OTU744 fails the condition of a network-wide connector, with a Cvalue of 0.58 . Yet with a Z-value, of $>1.7$, the OTU is a strong interaction partner to the host-plants within its module, which in this case is exclusively D. subulatum. There is with no indication of treatment effect on the strength of the interactions. Thus this OTU is a strong predominantly specialist and integral interaction partner of $D$. subulatum.

There was a relatively even distribution of ErM OTUs either side of the C-value threshold, this suggests that the ErM OTU trophic level has an equal proportion of generalist and specialists (Figure 33). Furthermore, this indicates that total network connectivity across species in the ErM network as the major contributor of its robustness. This indicates that the network is a more robust as a whole, rather than as separated species-specific sub-set networks. In addition, there was a relatively even degree of OTUs with negative and positive Z-values. Z-values below 0 indicate relative rarity of the OTUs in the overall network. 


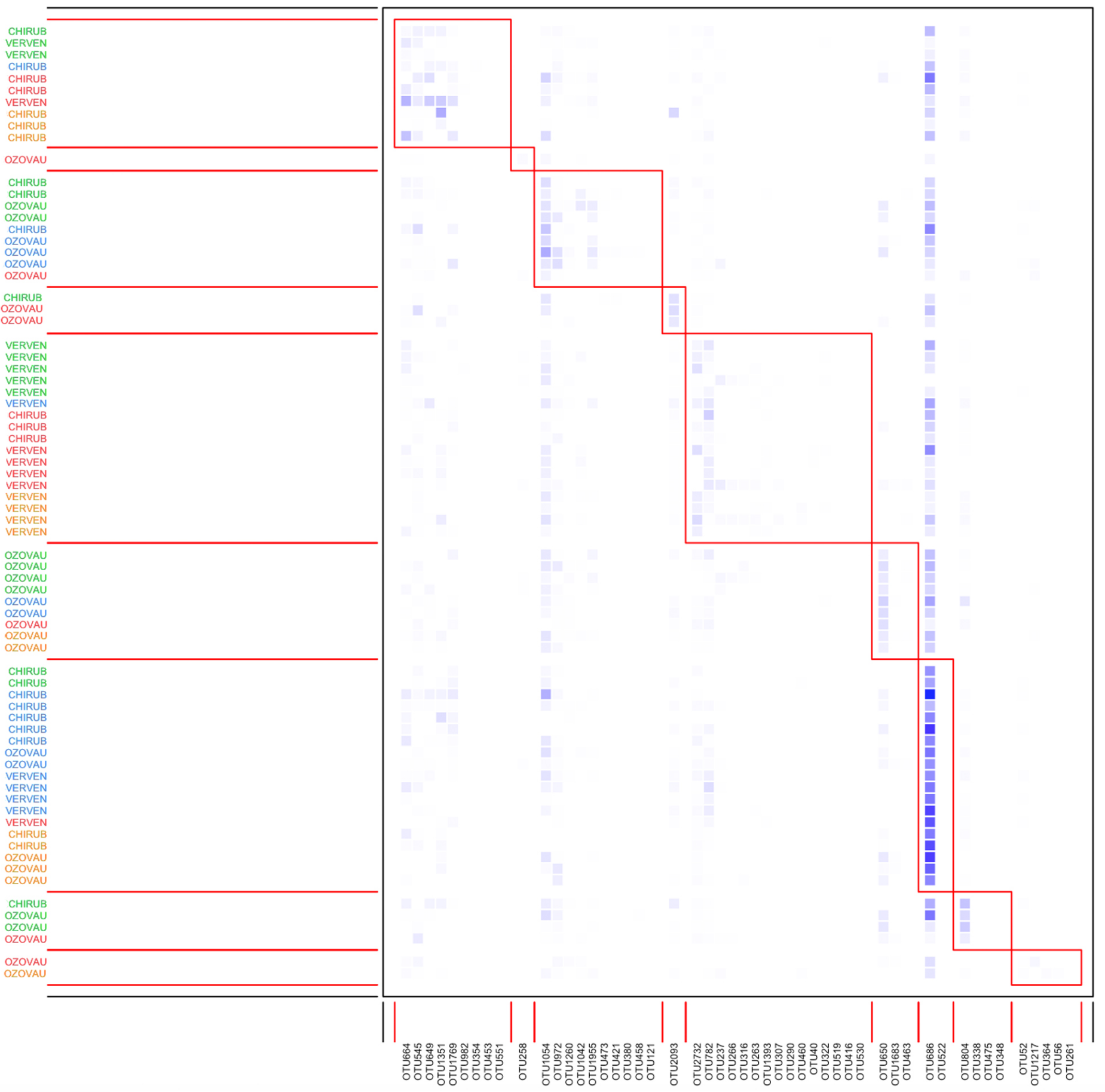

$\square$ ambient-control $\square$ ambient-removal $\square$ warm-control $\square$ warm-removal

Figure 34. Interaction matrix featuring modules of the arbuscular mycorrhizal network Modules identified by LPAwb+ method (Beckett, 2016), using computemodules function in the bipartite package in R. Q (modularity) $=0.232$. Darker squares indicate more observed interactions. Red boxes delineate the nine modules found. CHIRUB, Chionochloa rubra. OZOVAU, Ozothamnus vauvilliersii. VERVEN, Veronica venustula. 
The AM network scored a $\mathrm{Q}$ value of 0.23 , indicating that modules were strongly defined relative to most complex ecological networks. Due to the different number of interacting partners and interaction strengths, the $\mathrm{Q}$ values and the number of modules found in the ErM and AM networks can not be used for comparison in their structural integrity. No modules were defined by plot or treatment in the AM network.

The AM network was less connected than the ErM network, with few MF OTUs exhibiting generalist interactions consistently across modules. Figure 34 also shows there is comparably less strength in the interactions, with OTU686 and OTU1054 showing the highest strength. OTU686 and OTU1054 also exhibited a high level of between module connectivity. This indicates they are generalists responsible for a high proportion of the connectivity within the AM network. In addition, C. rubra shares modules with both $V$. venustula and $O$. vauvilliersii separately, which in turn only overlap in the module which is defined by interactions with generalist OTU686. This suggests that $V$. venustula and $O$. vauvilliersii do not necessarily benefit from network connectivity to each other, and thus fulfil diverse functional roles in the network. However, C. rubra presence benefits both $V$. venustula and $O$. vauvilliersii by sharing interaction partners and maintaining functional redundancy. In addition, $O$. vauvilliersii particularly in the warm-control treatment, does not exhibit similar interaction topologies, suggesting that this species may be opportunistic in its mycorrhizal interactions. 


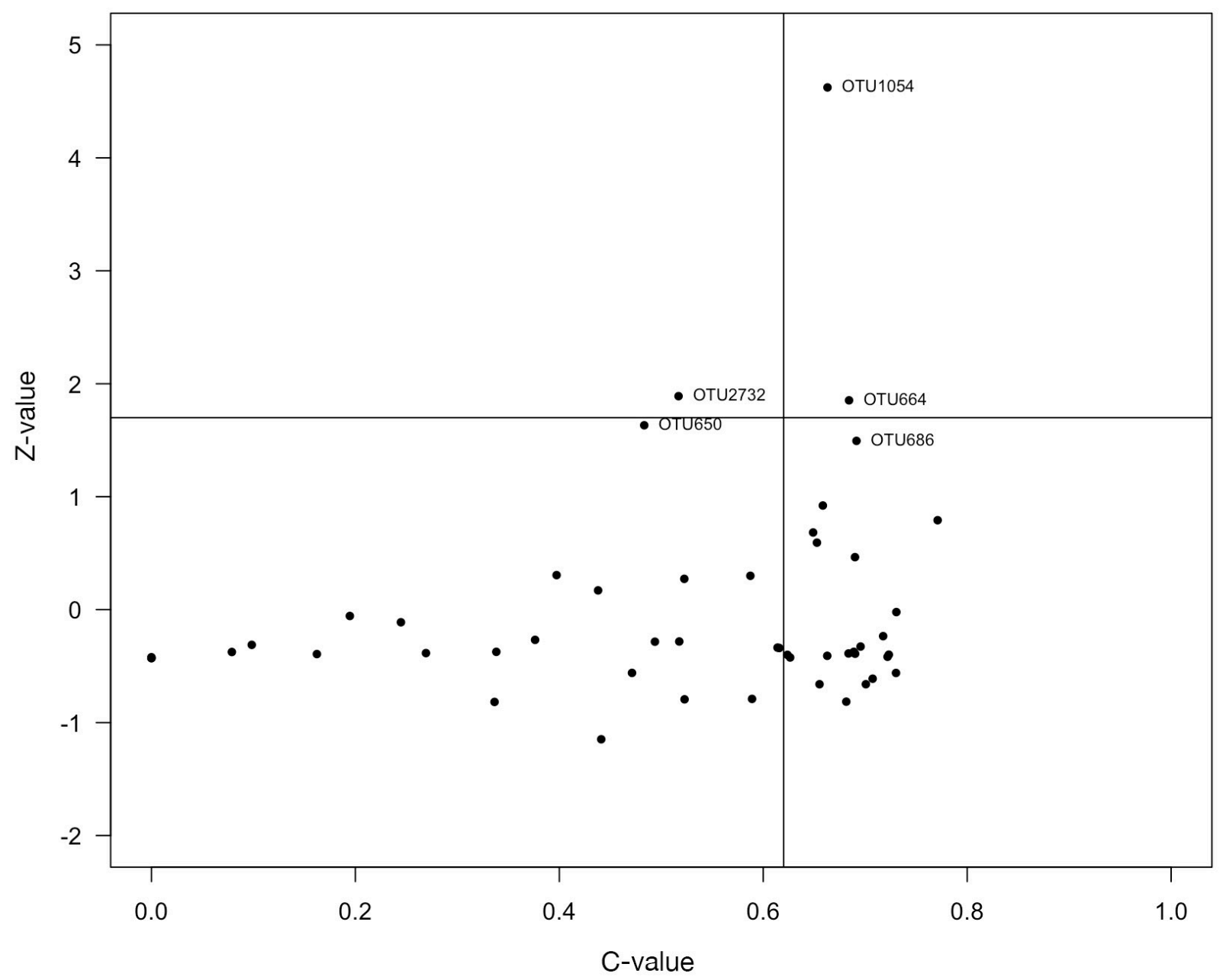

Figure 35. Connection $(C)$ and participation $(Z)$ values for arbuscular mycorrhizal fungi OTUs in the network of arbuscular-mycorrhizal host plant species. Values calculated using computemodules and czvalues function in the bipartite package in R. Black lines indicate critical C-value (Connection) $=0.62$ (Olesen et al., 2007) 0.62 and Z-value $($ Participation) $=1.7$ (for bipartite networks see Dormann and Strauss (2014). Top right quantile indicates OTUs exceeded both thresholds and are considered network "hub" species. CHIRUB, Chionochloa rubra. OZOVAU, Ozothamnus vauvilliersii. VERVEN, Veronica venustula

OTU1054 exhibited the highest level of combined among-module (C-values) and within-module (Z-values) connectivity. OTU1054 had strong interactions with both $C$. rubra and $O$. vauvilliersii, especially in the ambient-control treatment, suggesting that warming affects the overall connectivity and co-occurance OTUs within the same module. Conversely, OTU664 had strong interactions with C. rubra and V. venustula, 
regardless of treatment. In addition, OTU686 fell short of the Z-score threshold, suggesting it may function as a true generalist, and does not promote the strength of any particular module of the network. Conversely, OTU2732 and OTU650 promoted the strength of specialist modules of $V$. venustula and $O$. vauvilliersii respectively without increasing overall network connectivity between modules.

As there was a relatively even distribution of AM OTUs either side of the C-value threshold, this suggests that the AM OTU trophic level has an equal proportion of generalist and specialists (Figure 35). This also suggests maintaining AM network connectivity is a key component of its robustness, particularly due to the proportionally higher generality of C. rubra. However, in comparison to the ErM network there appears to be a higher degree of specialists existing within modules of the AM network, indicating a higher degree of network fragmentation. In addition, there was a relatively higher degree of OTUs with negative than positive Z-values. Z-values below 0 indicate relative rarity in the overall network and suggests that the AM network has relative weaker interactions than the ErM network. 


\section{Discussion}

Here, I analysed the architecture of interactions of plant-mycorrhizal networks in response to the experimental warming and removal of a dominant invasive plant species. I found that warming led to significant declines in the connectivity, functional diversity and functional redundancy of interactions within the plant-MF network. My analyses also revealed that warming led to a significant increase in the influence of Calluna vulgaris on the network, while its removal elicited complex responses in the network structure. Modularity analysis confirmed that plant species were more functionally similar than experimental plots, bolstering the evidence for the significance in the strong treatment effects I detected within species.

At the network-level, warming and $C$. vulgaris removal treatments independently reduced the linkage density and weighted connectance metrics of networks. This indicates a reduction in the average connectivity of species to the network. As these metrics are network-averages, they do not reveal variability between and within the individual trophic levels. However, with 6 host plant species interacting with $100 \mathrm{MF}$ OTUs, these results are asymmetrically weighted, and overall, indicate an average decline in the diversity of MF interactions by host plant species. In a network context this means, on average, host plants are losing their connectivity to the MF trophic level under both treatments, regardless to changes in MF diversity. These results reflect the findings of reduced MF connectivity by invasive plant removals by Anderson et al., (2010) and the warming effects evidenced by Geml et al., (2015) However, the combined treatments (warm-removal) offset the independent effects of treatments and host plant connectivity 
returned to ambient-control levels. This indicates the effect of removal on the network was strongly dependent on the warming treatment.

On the other hand, C. vulgaris removal did not offset the warming-induced declines in the Shannon's diversity of interactions in the network. Interaction diversity is a proxy of overall community function as it represents that there is a higher diversity of interactions by species and in between species (Blüthegen and Klein, 2011; Cadotte et al., 2011). Although C. vulgaris removal was expected to reduce overall interaction diversity within the network, warming did not offset this effect. These results demonstrate that the networks under the ambient-control treatment are the most connected with the greatest diversity and cumulative network function. Whereas, warm-removal treatments, although equally connected between trophic levels as the ambient-control treatments, have lost cumulative network diversity function. This result suggests that $C$. vulgaris removal and warming treatment suppress network connectivity and functional diversity, reflecting the independent effects detected by Anderson et al., (2010) and Geml et al., (2015). However, the role of C. vulgaris in promoting interaction diversity within the network is offset by warming. Collectively, these results indicate that $C$. vulgaris significantly promotes, but warming significantly reduces, network connectivity and diversity. More significantly, warming offsets any positive effect that $C$. vulgaris has on these network properties. This evidence strongly supports my hypothesis that warming independently reduces the robustness and strength of the plant-mycorrhizal interaction network.

C. vulgaris had two opposing effects on the structure of plant mycorrhizal networks, on the one hand, I find strong evidence that it promotes the overall network 
functional diversity and connectivity, however simultaneously, it reduces the connectivity and centrality of native plants species, essentially displacing their roles in the network.

Evidence of $C$. vulgaris promoting network connectivity was exhibited by the significant reduction of network nestedness structure, due to removal treatment. In addition, C. vulgaris removal significantly affected interaction strength asymmetry and C-score indices. Additionally, these results imply that, on average, native host plant species are far less generalist than C. vulgaris as they exhibit a higher discrimination in the mycorrhizal interactions formed, these results reflect the findings by Awaydul et al., (2019) and the predictions of invasion success by mutualists by Richardson et al., (2007) Collectively, this suggests that $C$. vulgaris has a critical role in promoting network connectivity and that its interactions are typical of those of a successful invader, namely that it is a generalist that interacts with other generalists (Orlovich and Cairney, 2004; Allen et al., 2016; Duell et al., 2016; Moora et al., 2011). This is evidence in support of my hypothesis that $C$. vulgaris functions as a mycorrhiza generalist with a high influence on the network structure. C. vulgaris' role as a mycorrhizal network generalist also aligns with its role as a generalist in the plant-pollinator network, both in Tongariro National Park (Giejsztowt et al., 2020) and Cairngorms National Park (Devoto et al., 2012).

Modularity analysis also revealed that $C$. vulgaris forms a unique module of interactions, indicating that it has a distinctly different function to the native ErM host plants. Independent investigations of invasion-mutualist success have also identified that distinct differences in interaction function by invaders is a key component of their dominance in a community (Richardson et al., 2007; Zhang et al., 2010). In particular, 
my findings align with that of Allen et al., (2020) which identified an invasive species had significantly stronger mutualist interactions within the network compared to native host plants, despite no evidence of co-invasion of specialists. This evidence is further supported as all the $\mathrm{MF}$ in the $C$. vulgaris module were mainly strong generalists too, with $\mathrm{C}$-values $>0.62$, and $\mathrm{Z}$-values $<1.70$, indicating they promote network connectivity to an even greater degree than C. vulgaris. The only exception was OTU739 which has a Z-value $>1.70$, but also possess a $\mathrm{C}$-values $>0.62$, proving that although it forms strong interactions frequently with all C. vulgaris sampled, its ongoing presence in the network is not dependent on its interaction with the invader. These results are strong evidence that the success of $C$. vulgaris invasion is not a result of successful co-invasion with its own MF, but rather due to its functions as a network generalist. These findings align with previous work suggesting that the generalist characteristics of $C$. vulgaris enable its invasion (Grove et al., (2017), Duell et al., (2016), Orlovich and Cairney (2004).

Furthermore this provides evidence that, despite its distinct network function, the dominance of $C$. vulgaris on the below ground network is not suggestive of a shift toward a taxonomic fungal composition that actively inhibits the ErM native hosts. Instead, this suggests evidence that $C$. vulgaris adhered to the enhanced mutualism hypothesis within ericoid common mycorrhizal network (Reinhart and Callaway, 2006). Particularly, this result implies a similar mechanism to that found within Allen et al., (2020) and Zhang et al., (2010) in which the invader exhibited stronger interactions within its mutualists than the native host plants, likely facilitating a positive feedback loop enhancing its dominance. 
Further evidence that $C$. vulgaris promoted the connectivity of the ErM network was that removal treatment reduced the togetherness in ErM MF. Togetherness is a metric of average functional overlap and therefore the result signifies that $C$. vulgaris removal reduces frequency of functionally redundant interactions between MF. While this further reinforces the role of C. vulgaris as a generalist, it also implies that the MF trophic level is more functionally fragmented due to $C$. vulgaris removal, which is further intensified by warming treatment. High levels of network fragmentation due to C. vulgaris removal under warming was also confirmed by the measure of functional complementarity at both trophic levels, which declined under the combination of both treatments. This result is reflective of Anderson et al., (2010) and Bret-Harte et al., (2004) which found invasive species removal fragmented the below ground network and reduced community functions. Collectively, these findings support that $C$. vulgaris had a critical role in promoting functionally diverse MF even under warming treatment, and confirms $C$. vulgaris acts to enhance the plant-mycorrhizal mutualism at this site, as per Reinhart and Callaway's (2006) hypothesis.

In contrast to its role as a mutualism enhancer, I also reveal strong evidence that C. vulgaris reduces network robustness by reducing the connectivity and influence of native plants. For example, removal treatment significantly increased the weighted cluster coefficient scores of the network, which represents the average interaction strength between host plants with their MF. This suggests the presence of $C$. vulgaris significantly reduces the strength of interactions by native host plants, independent of warming treatment. Furthermore, C. vulgaris removal significantly increased the average 
vulnerability of host plants by increasing the extinction slope value. While this metric represents the highly unlikely event of host plant extinction due to the elimination of MF, it importantly quantifies there is greater evenness in the diversity of MF associated across all host plants. Furthermore, this implies some host plants species are more disproportionally disconnected to the MF trophic level due to the presence of $C$. vulgaris, particularly under warming. This is further reinforced by the increase in the total functional complementarity in the MF trophic level, which was then offset by warming treatment.

The species-specific effect of $C$. vulgaris on host plant connectivity was revealed within species-level analyses. C. vulgaris removal, independent of warming treatment, significantly increased the connectivity of $D$. subulatum and $O$. vauvilliersii, as evidenced in the calculation of normalised degree. Also $C$. vulgaris removal, independent of warming treatment, significantly increased the weighted closeness of $O$. vauvilliersii and $V$ venustula. In addition, removal treatment increased the species strength metrics of $V$. venustula. These metrics define species influence, by calculating their proportional interaction strength and diversity within the network. Changes within these metrics are therefore considered as shifts in "centrality" of the network. Therefore these analyses collectively conclude that the presence of $C$. vulgaris decentralises $D$. subulatum, $O$. vauvilliersii and $V$. venustula, independent of warming treatment. These results align with my hypothesis, that $C$. vulgaris removal increases the connectivity and robustness of the common arbuscular mycorrhizal network. This provides strong support of the degraded mutualism hypotheses by Vogelsang and Bever, (2009), as C. vulgaris has suppressed the 
interactions of both arbuscular mycorrhizal host plants and ericoid mycorrhizal interactions by $D$. subulatum. However, the benefit of the removal effect in the AM network is highly species and warming-treatment dependent.

Species-level analyses also revealed that warming treatment altered the species centrality within the network. In particular, warming treatment reduced the proportional connectivity of D. subulatum, $O$. vauvilliersii and $V$. venustula to the MF trophic level, while simultaneously promoting the connectivity of $C$. vulgaris, as show in the normalised degree metric. Warming also increased the degree of MF discrimination by $C$. rubra, which signifies that there were fewer of its AMF interaction partners available. Furthermore, warming reduced the interaction diversity of $D$. subulatum, as demonstrated by the effective partners and proportional generality. Additionally, removal treatment also reduced the effective partners and proportional generality of D. subulatum and E. alpina, which partially supports my hypothesis in that $C$. vulgaris removal will reduce the connectivity and robustness of the common ericoid mycorrhizal network. However, the warming treatment offset the removal effect on both ErM species.

Modularity analyses revealed that $D$. subulatum and E. alpina occupied a similar level of functional diversity and similarity to each another, suggesting that although $E$. alpina was more generalist than $D$. subulatum, they exhibit similar capacity for interaction plasticity under experimental treatments. By comparison, all C. vulgaris samples were restricted to a single module, suggesting that $C$. vulgaris remains functionally distinct from other ErM species. Modularity analysis of the AM network revealed that C. rubra exhibited a degree of functional plasticity as it shared modules 
with both $V$. venustula and $O$. vauvilliersii, while the interactions of these species only overlapped in the module that contained generalist OTU686. Overall, this suggests that the AM network is functionally diverse with a high reliance on the generalist function of its host plants and MF, particularly OTU1054, OTU664 and OTU686. Despite this, in comparison to the ErM network there appears to be a higher degree of specialists with Cvalues $<0.62$, existing within modules of the AM network, indicating a higher degree of network fragmentation.

Several independent lines of evidence suggest that the composition on plantmycorrhizal networks was driven by treatments and not significantly affected by experimental or sampling artefacts like the position of plots and particular neighbouring plants (Chagnon et al., 2020; Bogar and Kennedy, 2013). However, my results showed that the composition of both mycorrhizal networks were not significantly determined by plot or treatment, but corresponded strongly to host plants species. This result indicates that therefore the treatment effects observed with species are independent the spatial positioning of plots. Furthermore, my testing of all network analysis metrics against 5 independent null models indicate that the significant effects detected in my data were highly unlikely to be a result of implicit sampling bias. This indicates, in this network of study, host plants are the abiotic-sensitive drivers of diversity within the plant-MF community, according to the driver habitat hypothesis by Zobel and Öpik, (2014). However, high MF dependence is inherently expected of a plant-obligate mutualist network. Furthermore, the degree of dependence is likely accentuated as a product of my sampling design, as I sampled one host plant species per plot. 
I conclude that warming and $C$. vulgaris act to decentralise and reduce the connectivity of native host plants to the mycorrhizal network. Warming reduces the overall connectivity of host plants to the MF trophic level. Warming also reduced the diversity of MF available for D. subulatum and the AM host plants. Furthermore, although $C$. vulgaris supports the connectivity of ErM fungi to the network, particularly under warming, it suppresses the network connectivity and influence of native host plants. Therefore, my results align with the findings of Duell et al., (2016) and Giejsztowt et al., (2020) and that of Nuñez and Dickie, (2014). I conclude that warming facilitates and exacerbates the negative effects of species invasion on the native plant-mycorrhizal network. I also find evidence that aligns with the findings of Dickie et al., (2010), Zhang et al., (2010) and Grove et al., (2017) whereby the presence of an invasive species buffers warming-induced declines of the interaction network by promoting connectivity and diversity within its networked species. 


\section{Chapter IV.}

\section{Thesis Review, Discussion and Synthesis}

Review of Aims and Hypotheses

In Chapter 2, I aimed to understand how the mycorrhizal fungal community of native alpine plants change in composition and diversity in response to two major drivers of global environmental change, warming and invasion.

I asked: How does warming and the presence of an invasive plant species independently and interactively impact the composition of mycorrhizal communities associated with native and an invasive plant species?

I hypothesised: Warming will reduce the diversity and alter the composition of the mycorrhizal fungal community associated with native plants. I predicted that the removal of invasive $C$. vulgaris will reduce the diversity of mycorrhizal fungi associated with host plants that form ericoid mycorrhizas, but promote the diversity of arbuscular mycorrhizal fungi associated with native host plants that form arbuscular mycorrhizas. I predicted that $C$. vulgaris will be generalist in its interactions and host a high diversity of ericoid mycorrhizal fungi relative to native host plant species. 
In Chapter 3, I aimed to reveal how the architecture of plant-mycorrhizal networks change in response to warming and invasion.

I asked: How does warming and the presence of an invasive plant species independently and interactively effect the structure and robustness of alpine plantmycorrhizal networks?

In addition, I asked: Do the responses of ericoid and arbuscular mycorrhizal networks to warming and the presence of an invasive plant differ?

I hypothesised: Warming will independently reduce the strength and diversity of interactions of native plants with their mycorrhizal fungi. I predicted that the removal of invasive C. vulgaris would reduce the diversity and robustness of the common ericoid mycorrhizal network but increase the diversity and robustness of the common arbuscular mycorrhizal network. I predicted C. vulgaris would act as a generalist with a strong influence on the structure and robustness of the plant-mycorrhizal network. 


\section{Key Findings}

I found strong support for my hypotheses that warming acts to reduce the diversity of mycorrhizal fungi of native plants and alter the structure of plant-mycorrhizal networks at this alpine tussock grassland site. For example, in Chapter 2 I showed that warming led to significant reductions in the richness and diversity of mycorrhizal fungi associated with native hosts, while in Chapter 3, I showed that warming reduces the connectivity of the native plant species to the mycorrhizal fungal network. Interestingly, my data reveal the opposite effect of warming on invasive C. vulgaris. Warming increased the richness and diversity of ErM fungi associated with C. vulgaris and increased its connectivity to the ericoid mycorrhizal fungal trophic level. These findings align with other evidence that warming facilitates C. vulgaris invasion in Tongariro National Park, and may increase its dominance of interactions in this alpine plant community (Giejsztowt et al., 2020). My study extends this work, by providing strong empirical support for the idea that warming increases the dominance of C. vulgaris in mutualistic interaction networks in TNP. This was demonstrated by the increase in the centrality of $C$. vulgaris and the concurrent decentralisation of native D. subulatum, $V$. venustula, and $O$. vauvilliersii in plant-mycorrhizal networks with warming treatment.

The removal of the ericaceous invader, $C$. vulgaris, had complex effects on the plant-mycorrhizal community, particularly when combined with the warming treatment. Foremost, and in accordance with my predictions, the removal of $C$. vulgaris increased the mycorrhizal fungal diversity of native host plants that form arbuscular mycorrhizas. Furthermore, removal of C. vulgaris restored the warming-induced losses of connectivity 
of $V$. venustula, and $O$. vauvilliersii to their AMF networks. Thus my data on plantmycorrhizal networks in a native tussock grassland invaded by ericaceous $C$. vulgaris align with the degraded mutualism hypothesis, which was developed to explain the negative effect of non-mycorrhizal herbs on native species growth in dry California grasslands that is driven by plant-soil feedbacks (Vogelsang and Bever, 2009). Bunn et al., (2015) outlines literary evidence for this hypothesis, as non arbuscular mycorrhizal invasive plants have been evidenced to decrease AM fungal abundance due to alterations in nutrient uptake efficacy (Bunn et al., 2014), reduced competitiveness (Busby et al., 2013) and biotic resistance of native plant-mycorrhizal communities (Lankau, 2010). Overall these results support my initial hypotheses that the presence of invasive $C$. vulgaris will reduce the diversity and connectivity of the arbuscular mycorrhizal interactions within the alpine plant community.

In contrast to the overall negative effects of $C$. vulgaris on the plant-AMF network, C. vulgaris had mixed effects on the ericoid common mycorrhizal network. I found that C. vulgaris was a strong generalist in the plant-ErM network, sharing many interactionspartners with the native ErM host species, despite showing a functional distinction in the NMS and modularity analyses. When present, $C$. vulgaris significantly increased network connectivity of ErMF, mitigating network fragmentation under warming. My results also strengthen the consensus that higher generality of interactions is an integral component to invasion success within interaction networks dominated by invasive C. vulgaris (Orlovich and Cairney, 2004; Giejsztowt et al., 2020; Devoto et al., 2012). These findings align with the enhanced mutualism hypothesis, which proposes 
that exotic invader plants encounter better mutualists in their exotic range than in their native range, leading to an increased abundance of their associated mutualists in the community (Reinhart and Callaway, 2006). In addition my results aligned with the findings of Allen et al., (2020) which found that higher strength of interactions both mutualistic and antagonistic is an integral component to invasion success within interaction networks. On the other hand, the combination of $C$. vulgaris removal and warming treatment led to no change in ErM diversity or plant-ErM connectivity in both native ericaceous species, relative to the control, despite the significant negative effects of these treatments individually. This suggests that warming enhanced the recovery of native plant-ErM interaction networks when C. vulgaris was removed. ErM networks appeared comparatively more resilient than AM networks under warming, and even enjoyed some benefits. However, my thesis did not examine the mechanisms by which $C$. vulgaris or warming promotes or suppresses mycorrhizal interactions in plant species or how it impacts soil legacy effects. Yet my thesis is novel as it has considered the effect of the presence of an invasive species on a plant-mycorrhizal community consisting of two functional guilds of mycorrhizal interactions.

Collectively, these findings show that ErM and AM networks differed in their response to warming and the presence of in C. vulgaris. In summary, the diversity and connectivity AM networks decreased due to warming and increased due to the removal of C. vulgaris. Therefore, unlike the predictions of Bellgard and Williams, (2011) and Klink et al., (2020), my results do not suggest that AMF are extending in their dominance within an alpine region due to warming. Comparatively, ErM networks appeared more 
resilient than AM networks under warming, where the diversity and connectivity of ErMF associated with $C$. vulgaris under warming increased. In addition, despite $C$. vulgaris removal and warming treatments decreased the diversity and connectivity of the native ErM host plants, the combined treatment recovered these attributes mostly to ambient control levels. Furthermore, under the warm-removal treatment, despite similar levels of network connectivity between trophic levels under the ambient-control, the warming induced declines in normalised degree for $O$. vauvilliersii and $V$. venustula highlight that they are proportionally less connected to the trophic level. Although these are interesting insights, I have not found definitive evidence to conclude the ErM network collectively benefits from warming aside from C. vulgaris. However, these findings do reinforce the statements of Marín and Kahout, (2020), as my study shows that CMNs of different guilds have shown to vary in their response to drivers of change and disturbance types, implying significant future changes to the function of the plant community.

Instead I primarily found evidence that mycorrhizal fungal responses to the WaRM treatments were host plant species specific, rather than common within a mycorrhizal guild. This result was highlighted in the NMS analyses with revealed MF variation was best described by WaRM treatments within each host plant. In the context of the habitat driver-passenger hypothesis (Zobel and Öpik, (2014), these results suggest that host plants were the environmentally susceptible drivers of mycorrhizal interactions in this alpine tussock grassland. Furthermore, I show that the generality in interactions of host plants (e.g., C. rubra, E. alpina and C. vulgaris) is a fundamental component of resilience in mycorrhizal diversity in this system. Interaction generality in these host 
plants led to functional overlap and redundancy of plants available to interact with MF, ensuring continued connectivity of the network, despite warming induced declines.

My thesis demonstrates that two important global change drivers, warming and invasive plant species, independently and interactively effect native alpine plantmycorrhizal communities in Tongariro National Park, New Zealand. My results showed that warming overall reduced the diversity and connectivity in the mycorrhizal interactions in all host plants, and increased that in the invader, C. vulgaris. Furthermore my data suggests that the effect of $C$. vulgaris removal on the mycorrhizal community compositions is highly dependent on warming. Finally, I presented evidence that warming and the presence of $C$. vulgaris synergistically weaken the connectivity and the influence of native plant species on the mycorrhizal network. Therefore, I conclude that warming facilitates the invasion success of $C$. vulgaris, by means of promoting the strength of interactions with its mycorrhizal fungi and increasing its influence of the plant-mycorrhizal network. 


\section{Strengths}

One of the strengths of my study was the utilisation of the Warming and Removal in Mountains (WaRM) experiment in Tongariro National Park. The design allowed the sampling of 8 replicate plant-mycorrhizal interaction networks for each of the four treatments. The factorial design enabled the testing of both the independent and interactive effects of warming and the presence of an invasive species. In addition, each of the 32 plots in the TNP WaRM experimental site were randomly assigned a treatment, which avoids the misrepresentation of variance associated with underlying environmental gradients being interpreted as treatment effects. The response of plant-mycorrhizal fungal communities and interaction networks to long-term warming (Deslippe et al., 2012; Geml et al., 2015) and plant invasions (Grove et al., 2017; Duell et al., 2016) have been explored previously. However, very few studies have employed a long-term manipulative experiment to investigate the interaction of these effects on a naturally assembled plantmycorrhizal community. Furthermore, very few studies on the plant-below ground networks test plant-MF community responses across multiple replicates (Chagnon, 2016). My examination of plant-mycorrhizal networks in a replicated and factorial field experiment is a major strength of my thesis.

A novel aspect of my study is the concurrent examination of the response of two plant-mycorrhizal guilds (i.e., AM and ErM) to the experimental treatments. The significance of this is that the mycorrhizal functional types of host plant species have been highlighted as a primary determinant of plant-MF community structure across a variety of experiments (Chagnon et al., 2020; Bogar and Kennedy, 2013; Bunn et al., 
2015; Hausmann and Hawkes, 2009; Urcelay et al., 2003). Likewise spatial variability in the composition of plant and mycorrhizal communities led to strong neighbourhood effects on the structure and composition of plant-mycorrhizal networks. An advantage of my analytical approach was that I tested for the prevalence of neighbourhood effects through the inclusion of experimental plots as random factors in my linear models and multivariate analyses. For example, MRPP revealed strong effects of plant species and experimental treatments on MF community structure, but found no significant effect of plot. Additionally, the NMS ordinations accounted for a high proportion of total variance in the OTU composition of samples. These findings suggest that underlying environmental gradients or neutral processes (e.g., stochasticity in assembly), which may drive neighbourhood effects were small relative to treatment effects in my study. On the other hand, my NMS and modularity analyses indicate that mycorrhizal composition is highly dependent on host plant species, a finding that aligns with previous work (Chagnon et al., 2020, Toju and Sato, 2018). The consistency of treatment effects across host plant species enhances my confidence that the WaRM treatments were strong drivers of the composition and structure of the mycorrhizal community at this site.

My thesis both used novel approaches in its experimental design and statistical approach that have been seldom utilised in the investigation of plant-mycorrhizal communities. Furthermore, a fundamental strength of my analyses is determining consistent responses to experimental treatments across replicate networks. My analysis of network metrics were therefore capable of revealing consistent responses of the ecological community to experimental treatments (Dormann et al., 2017). The inclusion 
of network analyses revealed multiple facets of plant-mycorrhizal community response. The calculation of multiple metrics allowed for the delineation of treatment effects on the interaction network. In particular this gave further insight into the different mechanisms that were eliciting changes in network connectivity, mycorrhizal diversity and parter discrimination. However, the calculation of network metrics involves the simplification of many interactions into a single response variable, and the resulting value of a metric is meaningless in and of itself but is very useful in when considered relative to other values for comparable samples. Randomisations of my interaction matrices allowed me to assess the statistical significance of my findings by randomly generating 1000 replicates of interaction network matrices, derived from my sequence read data matrix. These randomly generated matrices were used as null model networks in which I tested for the significance of "treatment" effects in all network-level, trophic-level and special-level metrics I calculated. As I found no significant effects across all null model networks, this reinforces that the significant effects found in the sampled network were not due to random chance or a result of underlying bias within the data. Furthermore, I am confident I have captured the total MF diversity within the species and the treatments through testing MF OTU turnover within these data subsets with utilised rarefaction curve analyses. 


\section{Weaknesses}

One of the limitations of my study is that although Open-Top Chambers (OTCs) are considered as a highly practical method for passive warming in experimental plots, (Marion et al., 1997) like all tools of experimental manipulation, they produce specific artefacts. Marion et al., (1997) finds that OTCs significantly decrease wind chill factor and slightly increase grazer pressure in the plot, which has been attributed to changing plant function and decreasing mycorrhizal fungal colonisation (Bethlenfalvay et al., 1984). Flanagan et al., (2013) also details the tendency for OTCs to intensify soil disturbance when installed in areas of strong winds. Furthermore, soil disturbance has been evidenced to significantly impact the external hyphal growth in arbuscular mycorrhizal fungi (Jasper et al., 1989). However, it is likely that soil disturbance by OTCs is localised to the edge of the chambers. Notably, the WaRM study site in Rangipo Desert of Tongariro National Park is subjected to high winds, typical of an alpine desert, and it experiences with grazing pressure from an range of vertebrate and insect herbivores, including hares and rabbits (Horne, 1979. In addition, OTCs are not an accurate proxy for total climate warming effects, as they do not alter precipitation patterns, nor simulate changes to soil moisture associated with warming (Marion et al., 1997). Importantly, AMF root colonisation and the diversity in mycorrhizal fungal spore production has been found to significantly respond independently to both temperature and soil moisture (Cheng et al., 2016, Sun et al., 2013). Finally, as they only cover an area of $2.3 \mathrm{~m}^{2}$, utilising OTCs as a warming treatment may underrepresent the impacts of climate warming on the plant-MF community. In addition, as explored by Sherrer and 
Körner, (2009) climate warming in alpine regions manifests in a mosaic effect on the surface temperature of the landscape. Therefore, although my results represent the response of the plant-mycorrhizal fungal community to warming, there is a definite possibility for its underestimation and simplification of climate warming effects.

Species removal experiments also come with their particular artefacts. As an experimental tool, species removal allows for studies of naturally assembled communities. However, removals do not simulate a species absence from the community, but are only true of an effect of how long that species has been removed. Within my study Calluna vulgaris removal is maintained by clipping its above ground biomass and weeding the seedlings to create minimal soil disturbance. As such there is still remnants of below ground root biomass and thus soil legacy effects of Calluna vulgaris. These roots are presumed inactive in hosting mycorrhizal fungi as nutrient transfer is highly unlikely without photosynthesis, and AMF have only been found to maintain their storage organs, and ErM their hyphae, in dead roots for a maximum of 14 days (Müller et al., 2017). However, AMF, and in very rare circumstances ErMF, spores have been evidenced to remain dormant for years (Smith and Read, 2010). In addition, alpine plant growth rates are slow (Chapin and Körner, 2013) and therefore species removal experiments often record a significant lag in above and below ground biomass response (McLaren and Turkington, 2011).

The study site also covers a small spatial area, whereby in the sampling of one individual per host plant species within the plots, the experiment does not accurately represent the composition of plant community, or the variability across environmental 
gradients TNP (Giejsztowt et al., 2020). Therefore I have assumed the dominance of invasive C. vulgaris in TNP and formulated my conclusions based upon the findings of Giejsztowt et al., (2020). Moreover, sampling a greater diversity of host plant species has the potential to reveal different responses of the MF network to the WaRM treatments. However, the consistency of species-specificity found in the NMS analyses suggests I would continue to find mycorrhizal compositions to be highly dependent on host plants. Moreover, I could extend my analyses to examine the particular mechanisms by which warming and C. vulgaris is altering mycorrhizal interactions. Mechanisms to measure, particularly in regard to the enhanced and degraded mutualism hypothesis, would be changes within soil nutrient compositions, moisture and spatial competition.

My thesis also made reductive assumptions of the functions of host plant and mycorrhizal fungi within the wider ecological community. Foremost, my study only considered function to be the variation in interactions between host plants and mycorrhizal fungi. Other variation in the functions of plant-mycorrhizal interactions, such as mycorrhizal-specific variation in nutrient transfer rates (Guether et al., 2009) were not considered in my study. Moreover, I assumed that all ErMF on the EM host plants and AMF on the AM host plants formed equally mutualistic interactions, despite evidence of mycorrhizal associations found to exist upon a mutualism-parasitism continuum dependent on abiotic stressors (Johnson and Graham, 1997; Smith and Smith, 2013). Additionally, I made the assumption that AMF did not form consequential mycorrhizas on the ericaceous host plants and vice versa. To disentangle variation in mycorrhizal efficacy among interaction partners future work could track nutrient transfer between 
plant and $\mathrm{MF}$ using dual stable ${ }^{13} \mathrm{C}$ and nitrogen ${ }^{15} \mathrm{~N}$ isotope signatures (Gleixner et al., 1993; Klink et al., 2020).

Finally, my statistical analysis was limited in two ways. Firstly, because of the patchiness of alpine plant communities, I could not achieve even species sampling across all plots and treatments. Therefore, I could not achieve the maximum statistical power capable for a factorial experiment, as the data did not meet the assumptions for exhaustive testing through mixed linear models or multivariate analysis of variance (MANOVA). Secondly, two of my network analyses metrics, interaction strength asymmetry and species strength, measure a value of "dependency". Dependence analyses are the subject of heavy critique, particularly for asymmetric bipartite networks. Blüthgen, (2010) demonstrated that dependence in an asymmetric network can originate either from specialist interactions or network fragmentation. However, most of the available dependence metrics cannot distinguish between these two drivers of dependence. Disentangling these drivers of dependence is important in our understanding of mutualistic networks as specialism is a key indicator of network stability (McCann, 2000; Ferlian et al., 2018). Conscious of this, I calculated a myriad of metrics to cover multiple facets of network architecture. In particular, nestedness, the C-score and functional complementarity metrics revealed C. vulgaris functions as critical interaction partner for rare MF and retaining network connectivity under warming and therefore "dependence" was correctly observing these both properties in the network. 
Future Directions

To gain further insight on the response of the plant-mycorrhizal community in TNP, taking measurements of soil nutrient concentrations, moisture and soil $\mathrm{pH}$ will reveal information as to how these networks may be effecting ecosystem functions. This would be particularly insightful as ErMF are considered to possess the highest saprotrophic capabilities of the MF guilds, thus this may reveal an additional component to ErM network's comparative success over the AM network under warming (Smith and Read, 2010). In addition, expanding my sampling effort to confidently analyse the orchid mycorrhizal and ectomycorrhizal compositions in the community may further illuminate community responses to the treatment. Moreover, sampling plant-mycorrhizal networks and soil conditions at multiple time intervals will give a greater understanding to the temporal variation of MF within these networks (Grove et al., 2017).

Future study of plant-mycorrhizal interactions networks should integrate methods to confirm nutrient transfer between plant and mycorrhizal fungal OTUs. Methods such as the inoculation of dual stable isotope signatures (Gleixner et al., 1993; Klink et al., 2020) or microscopic detection of mycorrhiza formations may inform of the true nature the fungal interactions and their diversity in function. Additionally this will give greater insight into the occurrence of dual-colonisations by MF functional types on host plants, which although were detected within my system, were considered inconsequential. Moreover, studies should further consider interactions between host plants, such as competition and allelopathic effects, between co-occuring common mycorrhizal networks of different functional guilds to further understand their effects in the community. 
These approaches will also enable more robust network analyses as it will reinforce the accuracy of interactions measured within the sampled plant-MF community. Furthermore, as outlined by Wahl and Spiegelberger (2016) and Johnson and Graham, (2013), continued investigation of the mutualism-parasitism continuum detected within mycorrhizal interactions may further illuminate how plant communities may alter in function under changing environmental conditions. Additionally, thorough ecological studies of plant-mycorrhizal fungal interactions require a prioritisation in contributing functional information to expand the UNITE and FUNGUILD databases. Through the active and systematic contributions of functional guild information for fungal OTUs, this will allow for more accurate identification of fungal diversity and the nature of interactions between plants and their mycorrhizal fungi. Specifically, in my study the limited resolution of fungal OTUs may have masked other fungal community shifts in response to the WaRM treatments, as out of the 3067 OTUs found within my sequence data, 532 did not have functional information.

Future research should also focus on utilising long-term factorial manipulation experiments on naturally assembled plant-mycorrhizal communities across multiple ecosystems. Experiments across multiple systems can reveal how MF communities of different biomasses, mycorrhizal functional guilds and different climatic and seasonal conditions may respond to drivers of change. Furthermore, alpine regions are regarded as possessing comparatively low MF biomass and diversities (Steidinger et al., 2019), therefore analysing the response of plant-mycorrhizal communities within warmer 
climate ecosystems could highlight critical components of rhizosphere processes that may mitigate or accentuate drivers of change.

Currently there is an active effort to understand the synergy and mitigation of effects under interacting drivers of change (Mantyka-Pringle et al., 2012; Bonan, 2008), however there are very few studies pertaining to plant-mycorrhizal communities under the combination of these effects (Chagnon, 2016). Specifically, conservation management strategies for alpine plant communities will benefit from further understanding of how increased nutrient deposition, ecological restoration methods (i.e., removals or biocontrol agents) and physical disturbances, interact with effects of climate warming and species invasions. For example, climate-induced increases in wildfire disturbance within TNP have been speculated to promote $C$. vulgaris invasion through the disturbance of plant community compositions (Perry et al., 2014). In addition, both seasonal and incidental fire disturbances have caused long-term declines in the diversity of mycorrhizal-fungi within the soil surface (Pattinson et al., 1999). Thus the interaction between climate warming and fire and hydrological disturbances could have a different and significant effect to invasions on the nutrient cycling and other ecosystem functions of plantmycorrhizal communities. 


\section{Synthesis Statements}

My thesis presents evidence that warming and the presence of an invasive species both independently and interactively reduced the diversity and connectivity of mycorrhizal fungal interactions in a native plant community. Furthermore, I demonstrate that warming facilitates the dominance of an invasive species on a below ground network by promoting the diversity and connectivity of its mycorrhizal fungal interactions in the network.

In addition my analyses revealed that the presence of an ericaceous invasive species, Calluna vulgaris, maintained the connectivity within, but altered the function of the ericoid common mycorrhizal network. Simultaneously, the presence of Calluna vulgaris, reduced the function and connectivity within a co-occurring arbuscular common mycorrhizal network. These findings contribute to the discourse on degraded and enhanced mutualism hypotheses by providing evidence that an invasive species can suppress the mycorrhizal interactions and network centrality of native host plant species.

Overall, my thesis contributes to understanding of plant-mycorrhiza community structure and function by explicitly investigating the effects of two global change drivers in tandem, and in their interaction, on a native alpine plant community. In summary, my thesis demonstrates the below-ground interactions of alpine plant communities are destabilising under multiple interacting drivers of global environmental change. 


\section{Appendix 1. Supplementary Tables}

Supplementary Table 1.

Supplementary Table 2

Supplementary Table 3

Supplementary Table 4

Supplementary Table 5

Supplementary Table 6

Supplementary Table 7

Supplementary Table 8
List of primer sequences used in PCR for the amplification of the fungal ITS2 region amplification. 2 variants of ITS3 primers and 18 variants of ITS4 were selected to create 36 unique forward and reverse bar-coded PCR products. The primers selected were designed to target the ITS2 region $(250-300 \mathrm{bp})$ the optimal region for parsing fungal diversity from sequence data (Blaalid et al., 2013; Bazzicalupo et al., 2013)

Pairwise differences of Multiple Response Permutation Procedure (MRPP) of DRASUB, Dracophyllum subulatum $(\mathrm{n}=29)$ by ErM OTUs $(p=47)$ by treatment. Significant difference between treatments detected with MRPP are denoted with *** if $\mathrm{p}$ $<0.0005, * *$ if $\mathrm{p}<0.005, *$ if $\mathrm{p}<0.05$.

Pairwise differences of Multiple Response Permutation Procedure (MRPP) of EPAALP, Epacris alpina $(\mathrm{n}=24)$ by ErM OTUs $(\mathrm{p}=$ $47)$ by treatment. Significant difference between treatments detected with MRPP are denoted with $* * *$ if $\mathrm{p}<0.0005, * *$ if $\mathrm{p}$ $<0.005, *$ if $\mathrm{p}<0.05$.

Pairwise differences of Multiple Response Permutation Procedure (MRPP) of CHIRUB, Chionochloa rubra $(\mathbf{n}=\mathbf{2 4})$ by AMF OTUs $(\mathbf{p}=\mathbf{5 3})$ by treatment. Significant difference between treatments detected with MRPP are denoted with $* * *$ if $\mathrm{p}<0.0005, * *$ if $\mathrm{p}$ $<0.005, *$ if $\mathrm{p}<0.05$.

Pairwise differences of Multiple Response Permutation Procedure (MRPP) of OZOVAU, Ozothamnus vauvilliersii. $(\mathrm{n}=28)$ by AMF OTUs $(p=53)$ by treatment. Significant difference between treatments detected with MRPP are denoted with $* * *$ if $p$ $<0.0005, * *$ if $\mathrm{p}<0.005, *$ if $\mathrm{p}<0.05$.

Pairwise differences of Multiple Response Permutation Procedure (MRPP) of VERVEN, Veronica venustula $(\mathrm{n}=22)$ by AMF OTUs $(\mathrm{p}=53)$ by treatment. Significant difference between treatments detected with MRPP are denoted with $* * *$ if $p<0.0005$, $* *$ if $p$ $<0.005, *$ if $\mathrm{p}<0.05$

Two-Way ANOVA results of species-level "structure" metrics by host-plant species. CALVUL, Calluna vulgaris. DRASUB, Dracophyllum subulatum. EPAALP, Epacris alpina. CHIRUB, Chionochloa rubra. OZOVAU, Ozothamnus vauvilliersii. VERVEN, Veronica venustula. Significant treatment effects from the Two-Way ANOVA are denoted with $* * *$ if $\mathrm{p}<0.0005, * *$ if $\mathrm{p}$ $<0.005, *$ if $\mathrm{p}<0.05,{ }^{t}$ if $\mathrm{p}<0.10$.

Two-Way ANOVA results of species-level "robustness" metrics by host-plant species. CALVUL, Calluna vulgaris. DRASUB, Dracophyllum subulatum. EPAALP, Epacris alpina. CHIRUB, Chionochloa rubra. OZOVAU, Ozothamnus vauvilliersii. VERVEN, Veronica venustula. Significant treatment effects from the Two-Way ANOVA are denoted with $* * *$ if $p<0.0005, * *$ if $p$ $<0.005, *$ if $\mathrm{p}<0.05,{ }^{t}$ if $\mathrm{p}<0.10$. 
Supplementary Table 1. List of primer sequences used in PCR for the amplification of the fungal ITS2 region amplification. 2 variants of ITS3 primers and 18 variants of ITS4 were selected to create 36 unique forward and reverse bar-coded PCR products. The primers selected were designed to target the ITS2 region (250-300bp) the optimal region for parsing fungal diversity from sequence data (Blaalid et al., 2013; Bazzicalupo et al., 2013)

\begin{tabular}{|c|c|c|}
\hline PRIMER IDENTITY & BINDING REGION & BARCODE SEQUENCE \\
\hline FORWARD PRIMER 1 & ITS3 & ATCACG \\
\hline FORWARD PRIMER 2 & ITS3 & TTAGGC \\
\hline REVERSE PRIMER 3 & ITS4 & ACTGAT \\
\hline REVERSE PRIMER 4 & ITS4 & TGACCA \\
\hline REVERSE PRIMER 5 & ITS4 & ACAGTG \\
\hline REVERSE PRIMER 6 & ITS4 & TATAAT \\
\hline REVERSE PRIMER 7 & ITS4 & CAGATC \\
\hline REVERSE PRIMER 8 & ITS4 & ACTTGA \\
\hline REVERSE PRIMER 9 & ITS4 & CAACTA \\
\hline REVERSE PRIMER 10 & ITS4 & CATTTT \\
\hline REVERSE PRIMER 11 & ITS4 & GGCTAC \\
\hline REVERSE PRIMER 12 & ITS4 & CTTGTA \\
\hline REVERSE PRIMER 13 & ITS4 & AGTCAA \\
\hline REVERSE PRIMER 14 & ITS4 & AGTTCC \\
\hline REVERSE PRIMER 15 & ITS4 & CACGAT \\
\hline REVERSE PRIMER 16 & ITS4 & CCGTCC \\
\hline REVERSE PRIMER 17 & ITS4 & GTAGAG \\
\hline REVERSE PRIMER 18 & ITS4 & GTCCGC \\
\hline REVERSE PRIMER 19 & ITS4 & GTGAAA \\
\hline REVERSE PRIMER 20 & ITS4 & TCATTC \\
\hline REVERSE PRIMER 21 & ITS4 & GTTTCG \\
\hline
\end{tabular}


Supplementary Table 2. Pairwise differences of Multiple Response Permutation Procedure (MRPP) of DRASUB, Dracophyllum subulatum $(\mathbf{n}=\mathbf{2 9})$ by ErM OTUs $(\mathbf{p}=$ 47) by treatment. Significant difference between treatments detected with MRPP are denoted with $* * *$ if $\mathrm{p}<0.0005, * *$ if $\mathrm{p}<0.005, *$ if $\mathrm{p}<0.05$.

\begin{tabular}{|c|c|c|c|c|}
\hline TREATMENT & $\begin{array}{l}\text { AMBIENT } \\
\text { CONTROL }\end{array}$ & $\begin{array}{l}\text { AMBIENT } \\
\text { REMOVAL }\end{array}$ & $\begin{array}{c}\text { WARM } \\
\text { CONTROL }\end{array}$ & $\begin{array}{c}\text { WARM } \\
\text { REMOVAL }\end{array}$ \\
\hline $\begin{array}{l}\text { AMBIENT } \\
\text { CONTROL }\end{array}$ & - & $\begin{array}{c}\mathrm{A}=0.058 \\
\mathrm{p}=0.003^{* * *}\end{array}$ & $\begin{array}{c}\mathrm{A}=0.038 \\
\mathrm{p}=0.032^{*}\end{array}$ & $\begin{array}{l}A=0.003 \\
p=0.352\end{array}$ \\
\hline $\begin{array}{l}\text { AMBIENT } \\
\text { REMOVAL }\end{array}$ & $\begin{array}{c}\mathrm{A}=0.058 \\
\mathrm{p}<0.003^{* * *}\end{array}$ & - & $\begin{array}{c}\mathrm{A}=0.051 \\
\mathrm{p}=0.016^{* *}\end{array}$ & $\begin{array}{c}\mathrm{A}=0.049 \\
\mathrm{p}=0.016^{* *}\end{array}$ \\
\hline $\begin{array}{c}\text { WARM } \\
\text { CONTROL }\end{array}$ & $\begin{array}{c}\mathrm{A}=0.038 \\
\mathrm{p}=0.032^{*}\end{array}$ & $\begin{array}{c}\mathrm{A}=0.051 \\
\mathrm{p}=0.016^{* *}\end{array}$ & - & $\begin{array}{c}\mathrm{A}=-0.021 \\
\mathrm{p}=0.863\end{array}$ \\
\hline $\begin{array}{c}\text { WARM } \\
\text { REMOVAL }\end{array}$ & $\begin{array}{l}A=0.003 \\
p=0.352\end{array}$ & $\begin{array}{c}A=0.049 \\
p=0.016^{* *}\end{array}$ & $\begin{array}{c}A=-0.021 \\
p=0.863\end{array}$ & - \\
\hline
\end{tabular}

Supplementary Table 3. Pairwise differences of Multiple Response Permutation Procedure (MRPP) of EPAALP, Epacris alpina $(\mathbf{n}=\mathbf{2 4})$ by ErM OTUs $(\mathbf{p}=\mathbf{4 7})$ by treatment. Significant difference between treatments detected with MRPP are denoted with $* * *$ if $\mathrm{p}<0.0005, * *$ if $\mathrm{p}<0.005, *$ if $\mathrm{p}<0.05$.

\begin{tabular}{|c|c|c|c|c|}
\hline TREATMENT & $\begin{array}{l}\text { AMBIENT } \\
\text { CONTROL }\end{array}$ & $\begin{array}{l}\text { AMBIENT } \\
\text { REMOVAL }\end{array}$ & $\begin{array}{c}\text { WARM } \\
\text { CONTROL }\end{array}$ & $\begin{array}{c}\text { WARM } \\
\text { REMOVAL }\end{array}$ \\
\hline $\begin{array}{l}\text { AMBIENT } \\
\text { CONTROL }\end{array}$ & - & $\begin{array}{c}\mathrm{A}=0.09 \\
\mathrm{p}=0.008^{* *}\end{array}$ & $\begin{array}{c}\mathrm{A}=0.067 \\
\mathrm{p}=0.019^{* *}\end{array}$ & $\begin{array}{c}\mathrm{A}=0.061 \\
\mathrm{p}=0.016^{* *}\end{array}$ \\
\hline $\begin{array}{l}\text { AMBIENT } \\
\text { REMOVAL }\end{array}$ & $\begin{array}{c}\mathrm{A}=0.09 \\
\mathrm{p}=0.008^{* *}\end{array}$ & - & $\begin{array}{l}A=0.027 \\
p=0.072\end{array}$ & $\begin{array}{c}\mathrm{A}=0.035 \\
\mathrm{p}=0.040^{*} *\end{array}$ \\
\hline $\begin{array}{c}\text { WARM } \\
\text { CONTROL }\end{array}$ & $\begin{array}{c}\mathrm{A}=0.067 \\
\mathrm{p}=0.019^{* *}\end{array}$ & $\begin{array}{l}A=0.027 \\
p=0.072\end{array}$ & - & $\begin{array}{c}A=-0.008 \\
p=0.611\end{array}$ \\
\hline $\begin{array}{c}\text { WARM } \\
\text { REMOVAL }\end{array}$ & $\begin{array}{c}\mathrm{A}=0.061 \\
\mathrm{p}=0.016^{* *}\end{array}$ & $\begin{array}{c}\mathrm{A}=0.035 \\
\mathrm{p}=0.040^{* *}\end{array}$ & $\begin{array}{c}\mathrm{A}=-0.008 \\
\mathrm{p}=0.611\end{array}$ & - \\
\hline
\end{tabular}


Supplementary Table 4. Pairwise differences of Multiple Response Permutation Procedure (MRPP) of CHIRUB, Chionochloa rubra $(\mathbf{n}=\mathbf{2 4})$ by AMF OTUs $(\mathbf{p}=\mathbf{5 3})$ by treatment. Significant difference between treatments detected with MRPP are denoted with $* * *$ if $\mathrm{p}<0.0005, * *$ if $\mathrm{p}<0.005, *$ if $\mathrm{p}<0.05$.

\begin{tabular}{|c|c|c|c|c|}
\hline TREATMENT & $\begin{array}{l}\text { AMBIENT } \\
\text { CONTROL }\end{array}$ & $\begin{array}{l}\text { AMBIENT } \\
\text { REMOVAL }\end{array}$ & $\begin{array}{c}\text { WARM } \\
\text { CONTROL }\end{array}$ & $\begin{array}{c}\text { WARM } \\
\text { REMOVAL }\end{array}$ \\
\hline $\begin{array}{l}\text { AMBIENT } \\
\text { CONTROL }\end{array}$ & - & $\begin{array}{l}A=0.020 \\
p=0.128\end{array}$ & $\begin{array}{c}\mathrm{A}=0.074 \\
\mathrm{p}=0.005^{* *}\end{array}$ & $\begin{array}{c}\mathrm{A}=0.072 \\
\mathrm{p}=0.006^{*}\end{array}$ \\
\hline $\begin{array}{l}\text { AMBIENT } \\
\text { REMOVAL }\end{array}$ & $\begin{array}{l}\mathrm{A}=0.020 \\
\mathrm{p}=0.128\end{array}$ & - & $\begin{array}{c}\mathrm{A}=0.058 \\
\mathrm{p}=0.014^{*}\end{array}$ & $\begin{array}{c}\mathrm{A}=0.063 \\
\mathrm{p}=0.034^{*}\end{array}$ \\
\hline $\begin{array}{c}\text { WARM } \\
\text { CONTROL }\end{array}$ & $\begin{array}{c}\mathrm{A}=0.074 \\
\mathrm{p}=0.005^{*} *\end{array}$ & $\begin{array}{c}\mathrm{A}=0.058 \\
\mathrm{p}=0.014^{*}\end{array}$ & - & $\begin{array}{l}A=0.001 \\
p=0.431\end{array}$ \\
\hline $\begin{array}{c}\text { WARM } \\
\text { REMOVAL }\end{array}$ & $\begin{array}{c}A=0.072 \\
p=0.006^{*}\end{array}$ & $\begin{array}{c}\mathrm{A}=0.063 \\
\mathrm{p}=0.034^{*}\end{array}$ & $\begin{array}{l}A=0.001 \\
p=0.431\end{array}$ & - \\
\hline
\end{tabular}

Supplementary Table 5. Pairwise differences of Multiple Response Permutation Procedure (MRPP) of OZOVAU, Ozothamnus vauvilliersii. $(\mathbf{n}=\mathbf{2 8})$ by AMF OTUs $(\mathbf{p}=$ 53) by treatment. Significant difference between treatments detected with MRPP are denoted with $* * *$ if $\mathrm{p}<0.0005, * *$ if $\mathrm{p}<0.005, *$ if $\mathrm{p}<0.05$.

\begin{tabular}{|c|c|c|c|c|}
\hline TREATMENT & $\begin{array}{l}\text { AMBIENT } \\
\text { CONTROL }\end{array}$ & $\begin{array}{l}\text { AMBIENT } \\
\text { REMOVAL }\end{array}$ & $\begin{array}{c}\text { WARM } \\
\text { CONTROL }\end{array}$ & $\begin{array}{c}\text { WARM } \\
\text { REMOVAL }\end{array}$ \\
\hline $\begin{array}{l}\text { AMBIENT } \\
\text { CONTROL }\end{array}$ & - & $\begin{array}{l}A=0.021 \\
p=0.191\end{array}$ & $\begin{array}{c}\mathrm{A}=0.130 \\
\mathrm{p}=0.002 * *\end{array}$ & $\begin{array}{l}A=0.033 \\
p=0.102\end{array}$ \\
\hline $\begin{array}{l}\text { AMBIENT } \\
\text { REMOVAL }\end{array}$ & $\begin{array}{l}A=0.021 \\
p=0.191\end{array}$ & - & $\begin{array}{c}\mathrm{A}=0.224 \\
\mathrm{p}=0.002 * *\end{array}$ & $\begin{aligned} A & =-0.023 \\
p & =0.596\end{aligned}$ \\
\hline $\begin{array}{c}\text { WARM } \\
\text { CONTROL }\end{array}$ & $\begin{array}{c}\mathrm{A}=0.130 \\
\mathrm{p}=0.002 * *\end{array}$ & $\begin{array}{c}\mathrm{A}=0.224 \\
\mathrm{p}=0.002 * *\end{array}$ & - & $\begin{array}{c}\mathrm{A}=0.211 \\
\mathrm{p}=0.006^{*}\end{array}$ \\
\hline $\begin{array}{c}\text { WARM } \\
\text { REMOVAL }\end{array}$ & $\begin{array}{l}A=0.028 \\
p=0.187\end{array}$ & $\begin{array}{c}A=-0.023 \\
p=0.596\end{array}$ & $\begin{array}{c}\mathrm{A}=0.211 \\
\mathrm{p}=0.006^{*}\end{array}$ & - \\
\hline
\end{tabular}


Supplementary Table 6. Pairwise differences of Multiple Response Permutation Procedure (MRPP) of VERVEN, Veronica venustula $(\mathbf{n}=\mathbf{2 2})$ by AMF OTUs $(\mathbf{p}=\mathbf{5 3})$ by treatment. Significant difference between treatments detected with MRPP are denoted with $* * *$ if $\mathrm{p}<0.0005, * *$ if $\mathrm{p}<0.005, *$ if $\mathrm{p}<0.05$.

\begin{tabular}{|c|c|c|c|c|}
\hline TREATMENT & $\begin{array}{l}\text { AMBIENT } \\
\text { CONTROL }\end{array}$ & $\begin{array}{l}\text { AMBIENT } \\
\text { REMOVAL }\end{array}$ & $\begin{array}{l}\text { WARM } \\
\text { CONTROL }\end{array}$ & $\begin{array}{c}\text { WARM } \\
\text { REMOVAL }\end{array}$ \\
\hline $\begin{array}{l}\text { AMBIENT } \\
\text { CONTROL }\end{array}$ & - & $\begin{array}{c}\mathrm{A}=0.029 \\
\mathrm{p}=0.044^{*}\end{array}$ & $\begin{array}{l}\mathrm{A}=0.042 \\
\mathrm{p}=0.014^{*}\end{array}$ & $\begin{array}{l}A=0.015 \\
p=0.132\end{array}$ \\
\hline $\begin{array}{l}\text { AMBIENT } \\
\text { REMOVAL }\end{array}$ & $\begin{array}{c}\mathrm{A}=0.029 \\
\mathrm{p}=0.044^{*}\end{array}$ & - & $\begin{array}{c}\mathrm{A}=0.081 \\
\mathrm{p}=0.003 * *\end{array}$ & $\begin{array}{l}\mathrm{A}=0.045 \\
\mathrm{p}=0.012^{*}\end{array}$ \\
\hline $\begin{array}{l}\text { WARM } \\
\text { CONTROL }\end{array}$ & $\begin{array}{c}\mathrm{A}=0.042 \\
\mathrm{p}=0.014^{*}\end{array}$ & $\begin{array}{c}\mathrm{A}=0.081 \\
\mathrm{p}=0.003^{*} *\end{array}$ & - & $\begin{array}{l}\mathrm{A}=0.035 \\
\mathrm{p}=0.012^{*}\end{array}$ \\
\hline $\begin{array}{c}\text { WARM } \\
\text { REMOVAL }\end{array}$ & $\begin{array}{l}A=0.015 \\
p=0.132\end{array}$ & $\begin{array}{c}\mathrm{A}=0.045 \\
\mathrm{p}=0.012 *\end{array}$ & $\begin{array}{c}\mathrm{A}=0.035 \\
\mathrm{p}=0.012^{*}\end{array}$ & - \\
\hline
\end{tabular}


Supplementary Table 7. Two-Way ANOVA results of species-level "structure" metrics by host-plant species. CALVUL, Calluna vulgaris. DRASUB, Dracophyllum subulatum. EPAALP, Epacris alpina. CHIRUB, Chionochloa rubra. OZOVAU, Ozothamnus vauvilliersii. VERVEN, Veronica venustula. Significant treatment effects from the TwoWay ANOVA are denoted with $* * *$ if $\mathrm{p}<0.0005, * *$ if $\mathrm{p}<0.005, *$ if $\mathrm{p}<0.05,{ }^{\prime}$ if $\mathrm{p}<0.10$.

\begin{tabular}{|c|c|c|c|c|}
\hline INDEX & SPECIES & WARMING & REMOVAL & WXR \\
\hline NORMALISED & CALVUL & $\begin{array}{r}\mathrm{F}=13.47 \\
\mathrm{p}=0.0032 * *\end{array}$ & N/A & N/A \\
\hline \multirow[t]{5}{*}{ DEGREE } & DRASUB & $\begin{array}{r}F=5.624 \\
p=0.0257 *\end{array}$ & $\begin{array}{c}\mathrm{F}=3.358 \\
\mathrm{p}=0.0788^{t}\end{array}$ & $\begin{array}{r}F=0.240 \\
p=0.6286\end{array}$ \\
\hline & EPAALP & $\begin{array}{l}F=0.000 \\
p=0.992\end{array}$ & $\begin{array}{l}F=0.119 \\
p=0.733\end{array}$ & $\begin{array}{l}F=2.897 \\
p=0.104\end{array}$ \\
\hline & CHIRUB & $\begin{array}{l}F=1.811 \\
p=0.193\end{array}$ & $\begin{array}{l}F=1.819 \\
p=0.193\end{array}$ & $\begin{array}{l}F=1.869 \\
p=0.187\end{array}$ \\
\hline & OZOVAU & $\begin{array}{r}\mathrm{F}=22.277 \\
\mathrm{p}<0.001 * * *\end{array}$ & $\begin{array}{r}\mathrm{F}=7.809 \\
\mathrm{p}=0.0101 \text { * }\end{array}$ & $\begin{array}{r}F=1.466 \\
p=0.2378\end{array}$ \\
\hline & VERVEN & $\begin{array}{r}\mathrm{F}=8.688 \\
\mathrm{p}=0.00861 * *\end{array}$ & $\begin{array}{r}F=2.740 \\
p=0.11519\end{array}$ & $\begin{array}{r}F=0.073 \\
p=0.78960\end{array}$ \\
\hline EFFECTIVE & CALVUL & $\begin{array}{l}F=2.143 \\
p=0.169\end{array}$ & N/A & N/A \\
\hline \multirow[t]{5}{*}{ PARTNERS } & DRASUB & $\begin{array}{r}\mathrm{F}=8.930 \\
\mathrm{p}=0.00621 * *\end{array}$ & $\begin{array}{r}F=0.546 \\
p=0.46678\end{array}$ & $\begin{aligned} & F=6.527 \\
& p=0.01709 *\end{aligned}$ \\
\hline & EPAALP & $\begin{array}{r}F=0.594 \\
p=0.44996\end{array}$ & $\begin{array}{r}F=1.153 \\
p=0.29568\end{array}$ & $\begin{array}{r}\mathrm{F}=9.839 \\
\mathrm{p}=0.0052 * *\end{array}$ \\
\hline & CHIRUB & $\begin{array}{l}F=0.129 \\
p=0.724\end{array}$ & $\begin{array}{l}F=0.935 \\
p=0.345\end{array}$ & $\begin{array}{l}F=2.199 \\
p=0.154\end{array}$ \\
\hline & OZOVAU & $\begin{array}{l}F=0.074 \\
p=0.788\end{array}$ & $\begin{array}{l}F=1.275 \\
p=0.270\end{array}$ & $\begin{array}{l}F=0.363 \\
p=0.553\end{array}$ \\
\hline & VERVEN & $\begin{array}{r}F=0.373 \\
p=0.5492\end{array}$ & $\begin{array}{r}\mathrm{F}=0.009 \\
\mathrm{p}=0.9235\end{array}$ & $\begin{array}{r}\mathrm{F}=8.188 \\
\mathrm{p}=0.0104\end{array}$ \\
\hline PROPORTIONAL & CALVUL & $\begin{array}{l}F=2.143 \\
p=0.169\end{array}$ & N/A & N/A \\
\hline \multirow[t]{5}{*}{ GENERALITY } & DRASUB & $\begin{array}{r}\mathrm{F}=8.930 \\
\mathrm{p}=0.00621 * *\end{array}$ & $\begin{array}{r}F=0.546 \\
p=0.46678\end{array}$ & $\begin{array}{r}\mathrm{F}=6.527 \\
\mathrm{p}=0.01709 *\end{array}$ \\
\hline & EPAALP & $\begin{array}{r}F=0.594 \\
p=0.44996\end{array}$ & $\begin{array}{r}F=1.153 \\
p=0.29568\end{array}$ & $\begin{array}{r}\mathrm{F}=9.839 \\
\mathrm{p}=0.0052 * *\end{array}$ \\
\hline & CHIRUB & $\begin{array}{l}F=0.129 \\
p=0.724\end{array}$ & $\begin{array}{l}F=0.935 \\
p=0.345\end{array}$ & $\begin{array}{l}F=2.199 \\
p=0.154\end{array}$ \\
\hline & OZOVAU & $\begin{array}{l}F=0.074 \\
p=0.788\end{array}$ & $\begin{array}{l}F=1.275 \\
p=0.270\end{array}$ & $\begin{array}{l}F=0.363 \\
p=0.553\end{array}$ \\
\hline & VERVEN & $\begin{array}{r}F=0.373 \\
p=0.5492\end{array}$ & $\begin{array}{r}F=0.009 \\
p=0.9235\end{array}$ & $\begin{array}{r}F=8.188 \\
p=0.0104 *\end{array}$ \\
\hline
\end{tabular}


Supplementary Table 8. Two-Way ANOVA results of species-level "robustness" metrics by host-plant species. CALVUL, Calluna vulgaris. DRASUB, Dracophyllum subulatum. EPAALP, Epacris alpina. CHIRUB, Chionochloa rubra. OZOVAU, Ozothamnus vauvilliersii. VERVEN, Veronica venustula. Significant treatment effects from the TwoWay ANOVA are denoted with $* * *$ if $\mathrm{p}<0.0005, * *$ if $\mathrm{p}<0.005, *$ if $\mathrm{p}<0.05,{ }^{t}$ if $\mathrm{p}<0.10$.

INDEX SPECIES WARMING REMOVAL WXR

\begin{tabular}{|c|c|c|c|c|}
\hline BLÜTHEGEN'S & CALVUL & $\begin{array}{l}F=0.241 \\
p=0.632\end{array}$ & N/A & N/A \\
\hline \multirow[t]{5}{*}{ D' INDEX } & DRASUB & $\begin{array}{l}F=0.151 \\
p=0.700\end{array}$ & $\begin{array}{l}F=1.753 \\
p=0.197\end{array}$ & $\begin{array}{l}F=2.198 \\
p=0.151\end{array}$ \\
\hline & EPAALP & $\begin{array}{r}F=1.076 \\
p=0.3119\end{array}$ & $\begin{array}{r}F=5.755 \\
p=0.0263 *\end{array}$ & $\begin{array}{r}F=0.037 \\
p=0.8497\end{array}$ \\
\hline & CHIRUB & $\begin{array}{r}\mathrm{F}=8.019 \\
\mathrm{p}=0.01031 *\end{array}$ & $\begin{array}{r}F=0.595 \\
p=0.44951\end{array}$ & $\begin{array}{r}\mathrm{F}=10.403 \\
\mathrm{p}=0.00424 * *\end{array}$ \\
\hline & OZOVAU & $\begin{array}{l}F=0.023 \\
p=0.880\end{array}$ & $\begin{array}{l}F=0.249 \\
p=0.622\end{array}$ & $\begin{array}{l}F=0.094 \\
p=0.762\end{array}$ \\
\hline & VERVEN & $\begin{array}{l}F=0.008 \\
p=0.932\end{array}$ & $\begin{array}{l}F=0.547 \\
p=0.469\end{array}$ & $\begin{array}{l}F=0.130 \\
p=0.722\end{array}$ \\
\hline WEIGHTED & CALVUL & $\begin{array}{r}\mathrm{F}=12.85 \\
\mathrm{p}=0.00376^{* *}\end{array}$ & N/A & N/A \\
\hline \multirow[t]{5}{*}{ CLOSENESS } & DRASUB & $\begin{array}{r}F=7.409 \\
p=0.0116^{*}\end{array}$ & $\begin{array}{r}\mathrm{F}=2.422 \\
\mathrm{p}=0.1322\end{array}$ & $\begin{array}{r}F=0.616 \\
p=0.4400\end{array}$ \\
\hline & EPAALP & $\begin{array}{l}F=0.086 \\
p=0.772\end{array}$ & $\begin{array}{l}F=1.096 \\
p=0.308\end{array}$ & $\begin{array}{l}F=0.980 \\
p=0.334\end{array}$ \\
\hline & CHIRUB & $\begin{array}{l}F=0.754 \\
p=0.396\end{array}$ & $\begin{array}{l}F=1.020 \\
p=0.324\end{array}$ & $\begin{array}{l}F=1.624 \\
p=0.217\end{array}$ \\
\hline & OZOVAU & $\begin{array}{r}\mathrm{F}=9.629 \\
\mathrm{p}=0.004852 * *\end{array}$ & $\begin{array}{r}\mathrm{F}=15.677 \\
\mathrm{p}<0.001^{* * *}\end{array}$ & $\begin{array}{r}F=2.728 \\
p=0.111611\end{array}$ \\
\hline & VERVEN & $\begin{array}{r}F=2.885 \\
p=0.106626\end{array}$ & $\begin{array}{r}\mathrm{F}=11.679 \\
\mathrm{p}=0.003070 * *\end{array}$ & $\begin{array}{r}\mathrm{F}=19.04 \\
\mathrm{p}<0.001 * * *\end{array}$ \\
\hline SPECIES & CALVUL & $\begin{array}{r}F=7.341 \\
p=0.019 *\end{array}$ & N/A & N/A \\
\hline \multirow[t]{5}{*}{ STRENGTH } & DRASUB & $\begin{array}{r}\mathrm{F}=8.497 \\
\mathrm{p}=0.0074 * *\end{array}$ & $\begin{array}{r}F=0.956 \\
p=0.3377\end{array}$ & $\begin{array}{r}F=0.209 \\
p=0.6519\end{array}$ \\
\hline & EPAALP & $\begin{array}{l}F=0.006 \\
p=0.937\end{array}$ & $\begin{array}{l}F=0.087 \\
p=0.772\end{array}$ & $\begin{array}{l}F=2.112 \\
p=0.162\end{array}$ \\
\hline & CHIRUB & $\begin{array}{l}F=0.475 \\
p=0.499\end{array}$ & $\begin{array}{l}F=1.802 \\
p=0.195\end{array}$ & $\begin{array}{l}F=0.883 \\
p=0.359\end{array}$ \\
\hline & OZOVAU & $\begin{array}{r}\mathrm{F}=4.953 \\
\mathrm{p}=0.0357 *\end{array}$ & $\begin{array}{r}F=0.386 \\
p=0.5401\end{array}$ & $\begin{array}{r}F=0.264 \\
p=0.6120\end{array}$ \\
\hline & VERVEN & $\begin{array}{r}\mathrm{F}=0.591 \\
\mathrm{p}=0.4520\end{array}$ & $\begin{array}{r}\mathrm{F}=4.418 \\
\mathrm{p}=0.049 *\end{array}$ & $\begin{array}{r}F=1.041 \\
p=0.3211\end{array}$ \\
\hline
\end{tabular}




\section{Appendix 2. Supplementary Figures}

Supplementary Bipartite plant-mycorrhizal networks by plots of the "ambientFigure 1 control" treatment $(\mathrm{n}=8)$ constructed with the plotweb function from the bipartite package in R. Host plants are on the "lower level" (lower trophic rank), connected to the ErM and AM fungal OTUs in the "higher level" (higher trophic rank). The width of the bar for each species (i.e., node) is proportional to the relative abundance of interactions it has, and acts as a proxy for the relative influence a species exerts on the network. The width of each link is proportional to the relative strength of the interaction, quantified as the number of fungal OTU sequence reads linking the fungal OTU and plant species nodes. Fungal OTUs are ordered from left to right firstly by ErM and AM fungi, and then in order of their overall rank abundance across all samples. CALVUL, Calluna vulgaris. DRASUB, Dracophyllum subulatum. EPAALP, Epacris alpina. CHIRUB, Chionochloa rubra. OZOVAU, Ozothamnus vauvilliersii. VERVEN, Veronica venustula.

Supplementary Bipartite plant-mycorrhizal networks by plots of the "ambientFigure 2 removal" treatment $(\mathrm{n}=8)$ constructed with the plotweb function from the bipartite package in R. Host plants are on the "lower level" (lower trophic rank), connected to the ErM and AM fungal OTUs in the "higher level" (higher trophic rank). The width of the bar for each species (i.e., node) is proportional to the relative abundance of interactions it has, and acts as a proxy for the relative influence a species exerts on the network. The width of each link is proportional to the relative strength of the interaction, quantified as the number of fungal OTU sequence reads linking the fungal OTU and plant species nodes. Fungal OTUs are ordered from left to right firstly by ErM and AM fungi, and then in order of their overall rank abundance across all samples. CALVUL, Calluna vulgaris. DRASUB, Dracophyllum subulatum. EPAALP, Epacris alpina. CHIRUB, Chionochloa rubra. OZOVAU, Ozothamnus vauvilliersii. VERVEN, Veronica venustula 
Supplementary Bipartite plant-mycorrhizal networks by plots of the "warmFigure 3 control" treatment $(\mathrm{n}=8)$ constructed with the plotweb function from the bipartite package in R. Host plants are on the "lower level" (lower trophic rank), connected to the ErM and AM fungal OTUs in the "higher level" (higher trophic rank). The width of the bar for each species (i.e., node) is proportional to the relative abundance of interactions it has, and acts as a proxy for the relative influence a species exerts on the network. The width of each link is proportional to the relative strength of the interaction, quantified as the number of fungal OTU sequence reads linking the fungal OTU and plant species nodes. Fungal OTUs are ordered from left to right firstly by ErM and AM fungi, and then in order of their overall rank abundance across all samples. CALVUL, Calluna vulgaris. DRASUB, Dracophyllum subulatum. EPAALP, Epacris alpina. CHIRUB, Chionochloa rubra. OZOVAU, Ozothamnus vauvilliersii. VERVEN, Veronica venustula

Supplementary Bipartite plant-mycorrhizal networks by plots of the "warmFigure 4 removal" treatment $(\mathrm{n}=8)$ constructed with the plotweb function from the bipartite package in R. Host plants are on the "lower level" (lower trophic rank), connected to the ErM and AM fungal OTUs in the "higher level" (higher trophic rank). The width of the bar for each species (i.e., node) is proportional to the relative abundance of interactions it has, and acts as a proxy for the relative influence a species exerts on the network. The width of each link is proportional to the relative strength of the interaction, quantified as the number of fungal OTU sequence reads linking the fungal OTU and plant species nodes. Fungal OTUs are ordered from left to right firstly by ErM and AM fungi, and then in order of their overall rank abundance across all samples. CALVUL, Calluna vulgaris. DRASUB, Dracophyllum subulatum. EPAALP, Epacris alpina. CHIRUB, Chionochloa rubra. OZOVAU, Ozothamnus vauvilliersii. VERVEN, Veronica venustula 


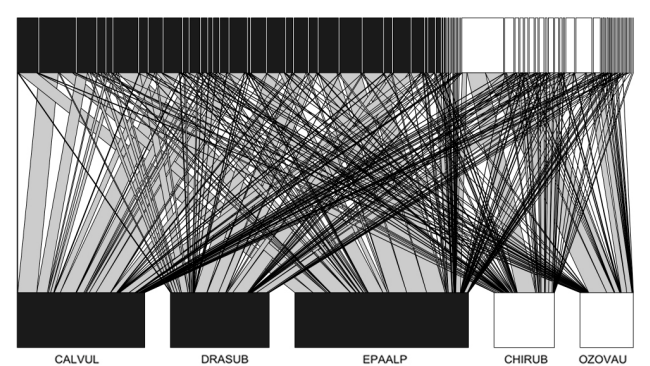

Plot 18

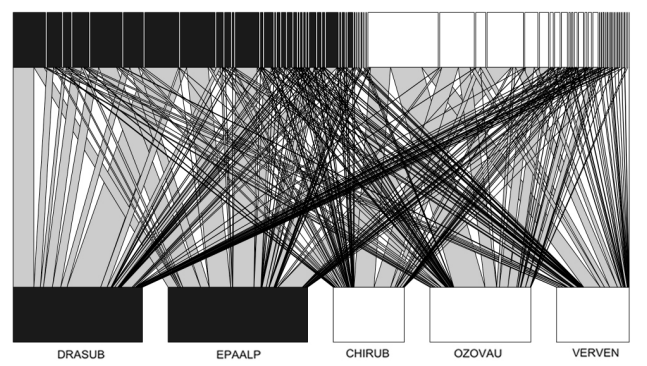

Plot 22

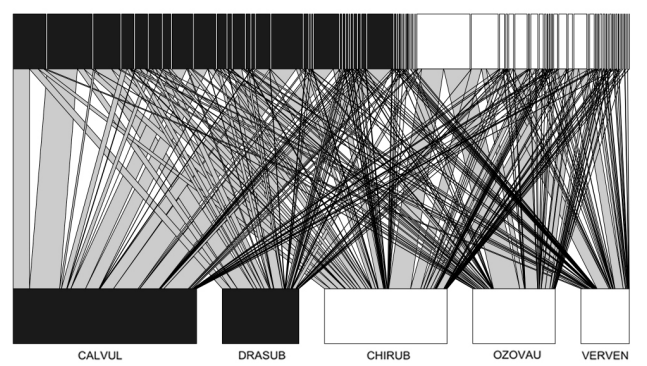

Plot 29

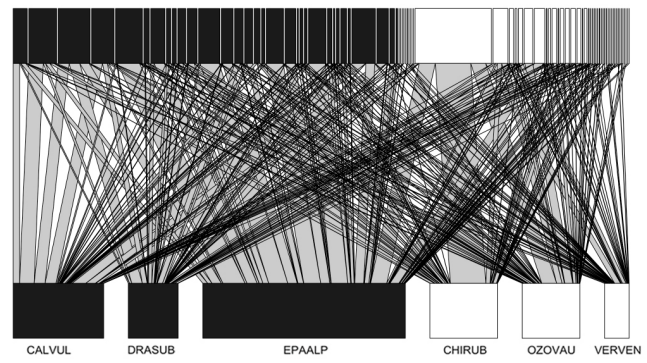

Plot 31

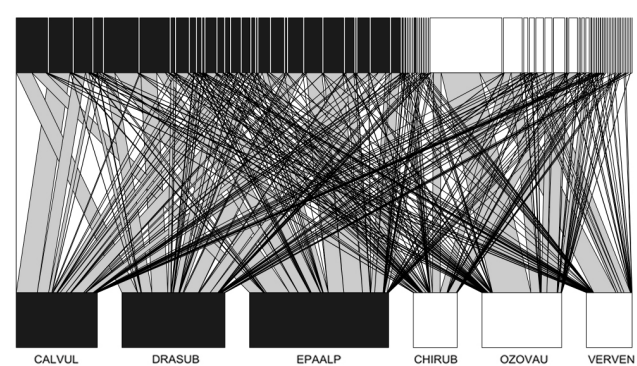

Plot 20

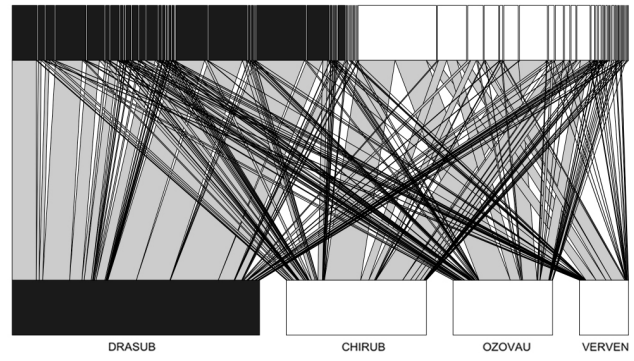

Plot 28

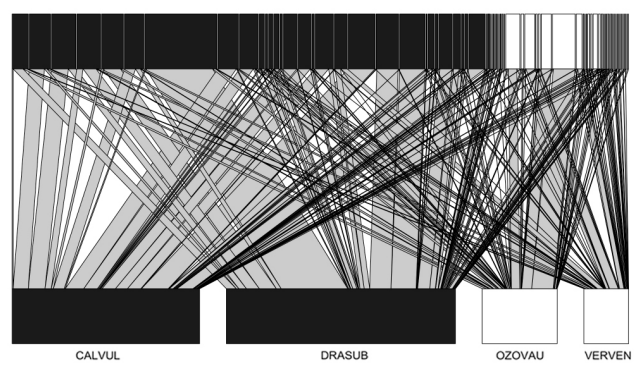

Plot 30

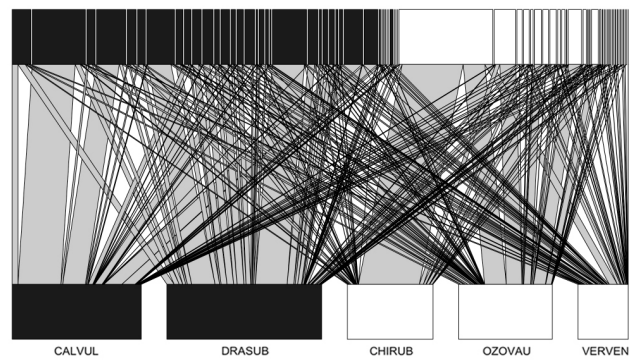

Plot 32

Supplementary Figure 1. Bipartite plant-mycorrhizal networks by plots of the "ambientcontrol" treatment $(\mathbf{n}=\mathbf{8})$ constructed with the plotweb function from the bipartite package in R. Host plants are on the "lower level" (lower trophic rank), connected to the ErM and AM fungal OTUs in the "higher level" (higher trophic rank). The width of the bar for each species (i.e., node) is proportional to the relative abundance of interactions it has, and acts as a proxy for the relative influence a species exerts on the network. The width of each link is proportional to the relative strength of the interaction, quantified as the number of fungal OTU sequence reads linking the fungal OTU and plant species nodes. Fungal OTUs are ordered from left to right firstly by ErM and AM fungi, and then in order of their overall rank abundance across all samples. CALVUL, Calluna vulgaris. DRASUB, Dracophyllum subulatum. EPAALP, Epacris alpina. CHIRUB, Chionochloa rubra. OZOVAU, Ozothamnus vauvilliersii. VERVEN, Veronica venustula. 


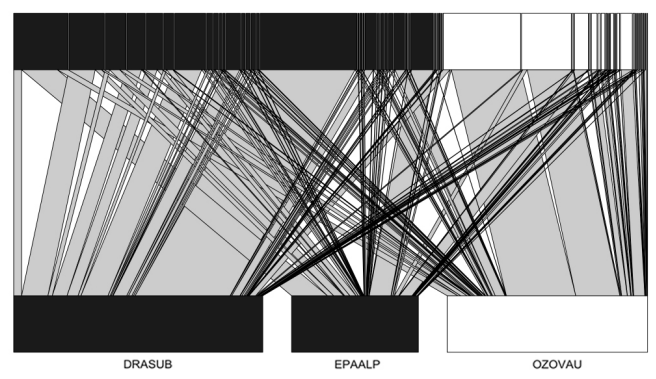

Plot 3

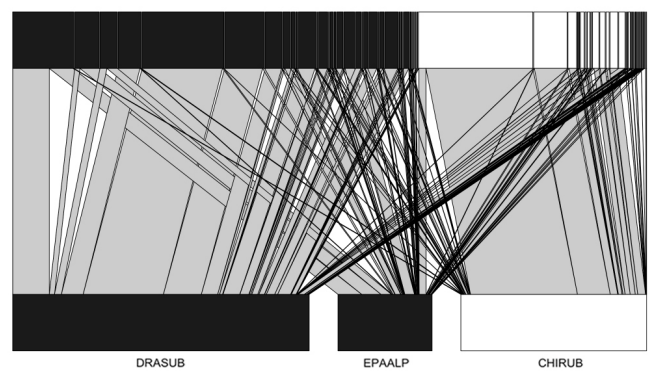

Plot 21

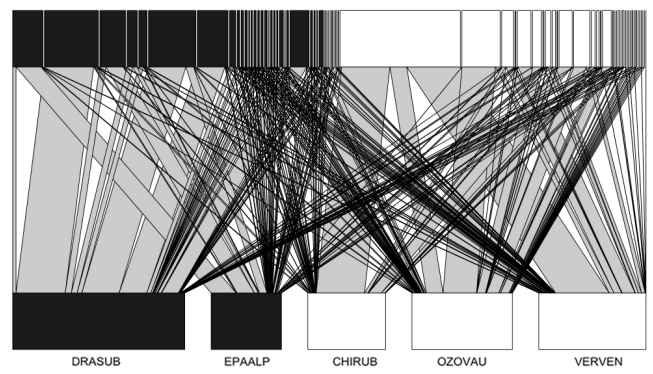

Plot 24

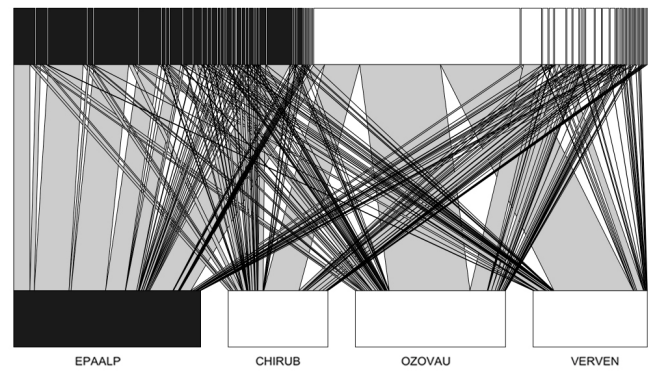

Plot 26

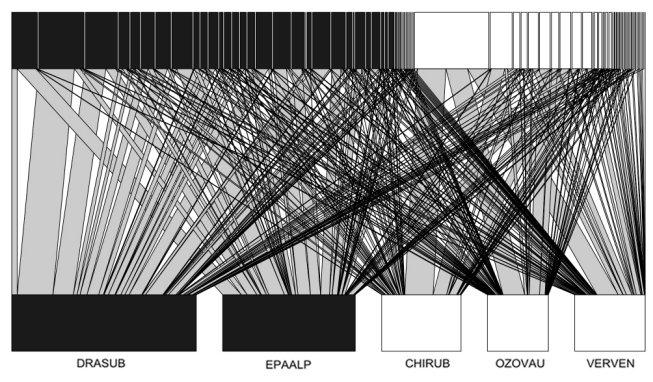

Plot 19

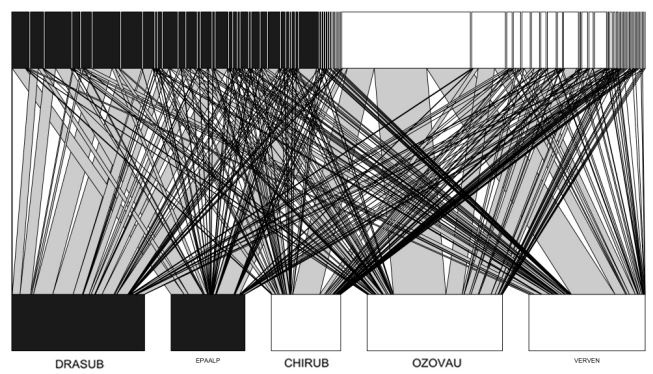

Plot 23

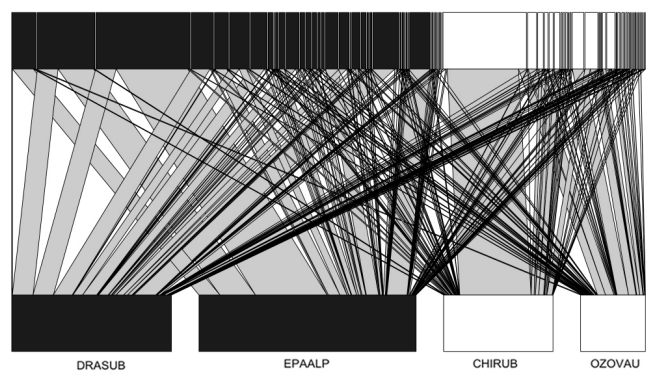

Plot 25

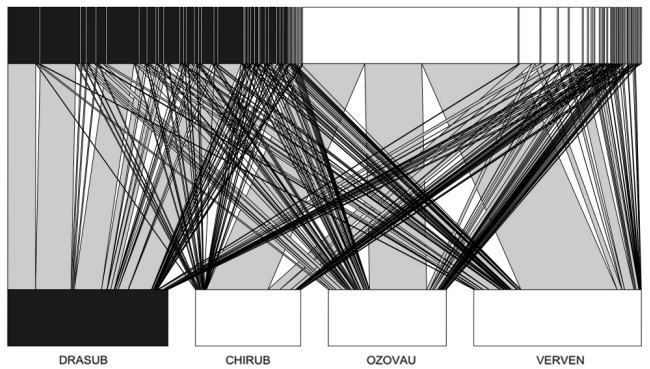

Plot 27

Supplementary Figure 2. Bipartite plant-mycorrhizal networks by plots of the "ambientremoval" treatment $(\mathbf{n}=\mathbf{8})$ constructed with the plotweb function from the bipartite package in R. Host plants are on the "lower level" (lower trophic rank), connected to the ErM and AM fungal OTUs in the "higher level" (higher trophic rank). The width of the bar for each species (i.e., node) is proportional to the relative abundance of interactions it has, and acts as a proxy for the relative influence a species exerts on the network. The width of each link is proportional to the relative strength of the interaction, quantified as the number of fungal OTU sequence reads linking the fungal OTU and plant species nodes. Fungal OTUs are ordered from left to right firstly by ErM and AM fungi, and then in order of their overall rank abundance across all samples. CALVUL, Calluna vulgaris. DRASUB, Dracophyllum subulatum. EPAALP, Epacris alpina. CHIRUB, Chionochloa rubra. OZOVAU, Ozothamnus vauvilliersii. VERVEN, Veronica venustula. 


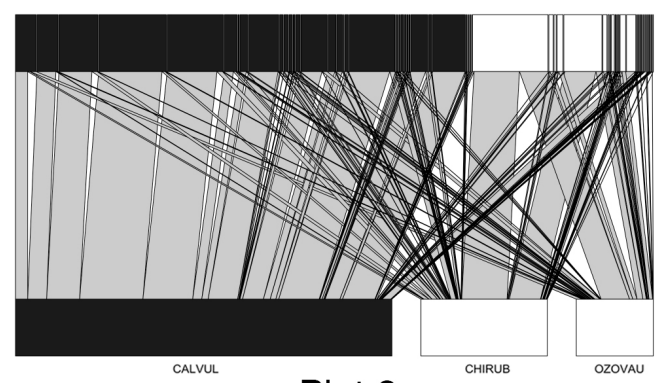

Plot 2

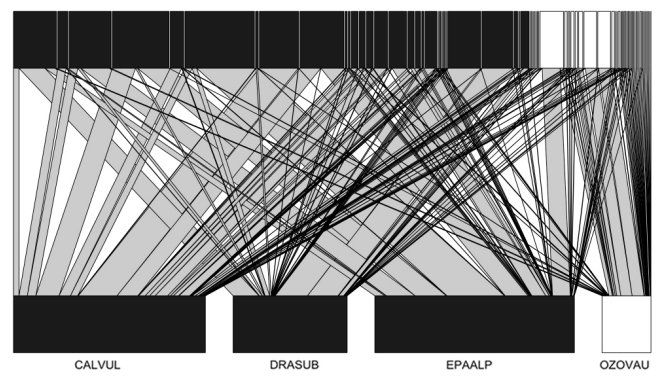

Plot 7

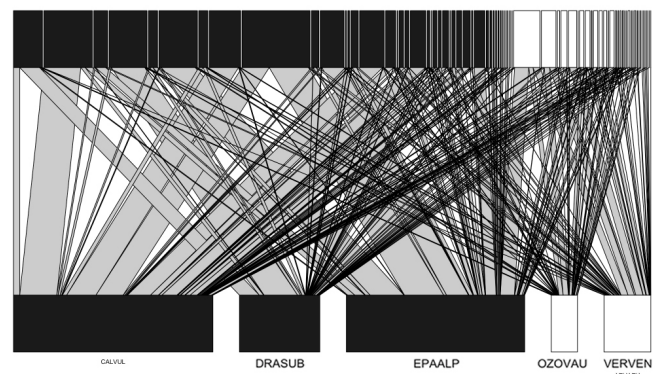

Plot 11

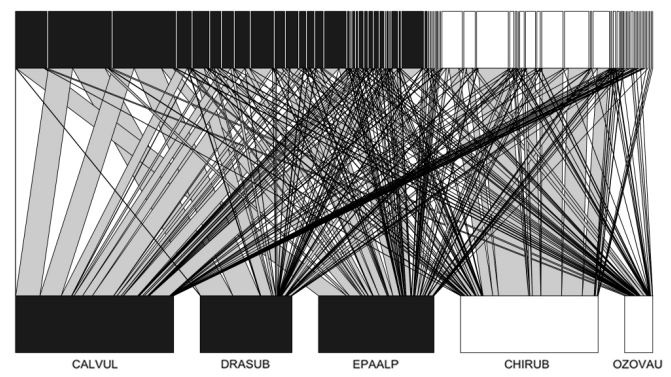

Plot 15

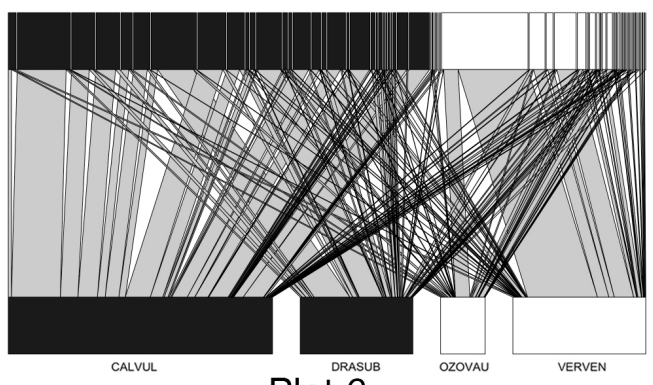

Plot 6

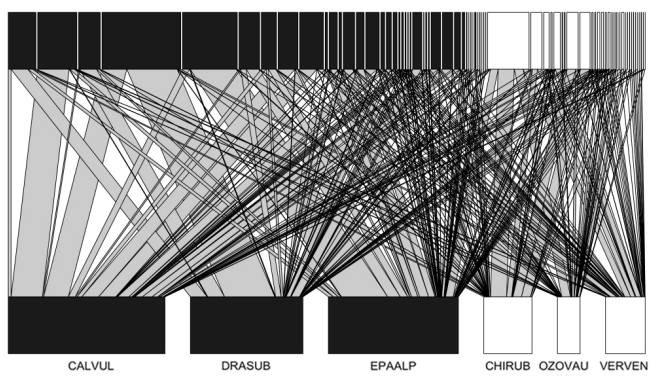

Plot 9

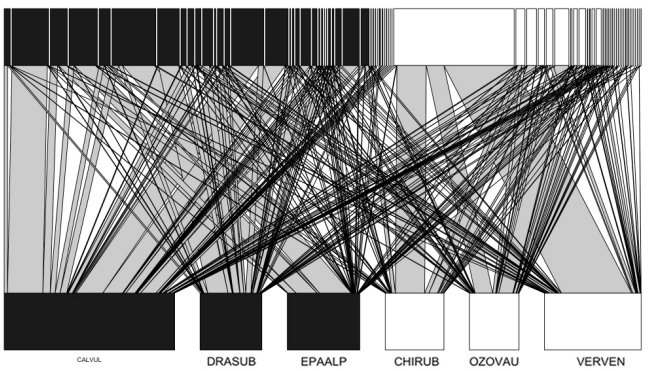

Plot 13

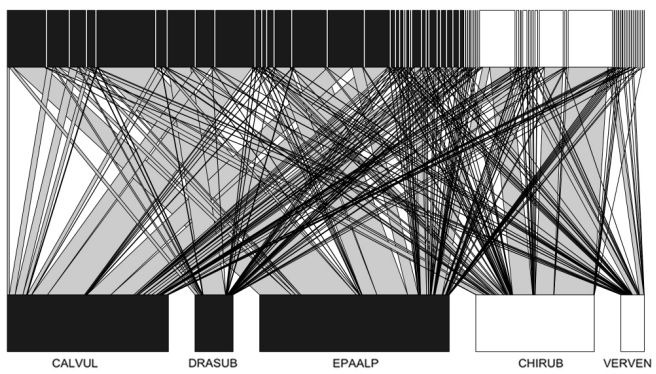

Plot 17

Supplementary Figure 3. Bipartite plant-mycorrhizal networks by plots of the "warmcontrol" treatment $(\mathbf{n}=\mathbf{8})$ constructed with the plotweb function from the bipartite package in R. Host plants are on the "lower level" (lower trophic rank), connected to the ErM and AM fungal OTUs in the "higher level" (higher trophic rank). The width of the bar for each species (i.e., node) is proportional to the relative abundance of interactions it has, and acts as a proxy for the relative influence a species exerts on the network. The width of each link is proportional to the relative strength of the interaction, quantified as the number of fungal OTU sequence reads linking the fungal OTU and plant species nodes. Fungal OTUs are ordered from left to right firstly by ErM and AM fungi, and then in order of their overall rank abundance across all samples. CALVUL, Calluna vulgaris. DRASUB, Dracophyllum subulatum. EPAALP, Epacris alpina. CHIRUB, Chionochloa rubra. OZOVAU, Ozothamnus vauvilliersii. VERVEN, Veronica venustula. 

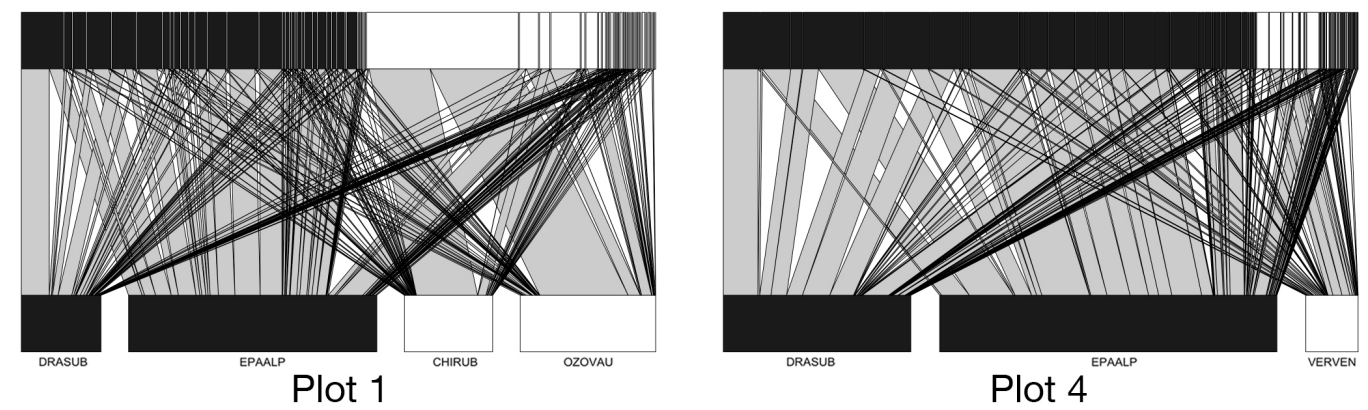

Plot 4
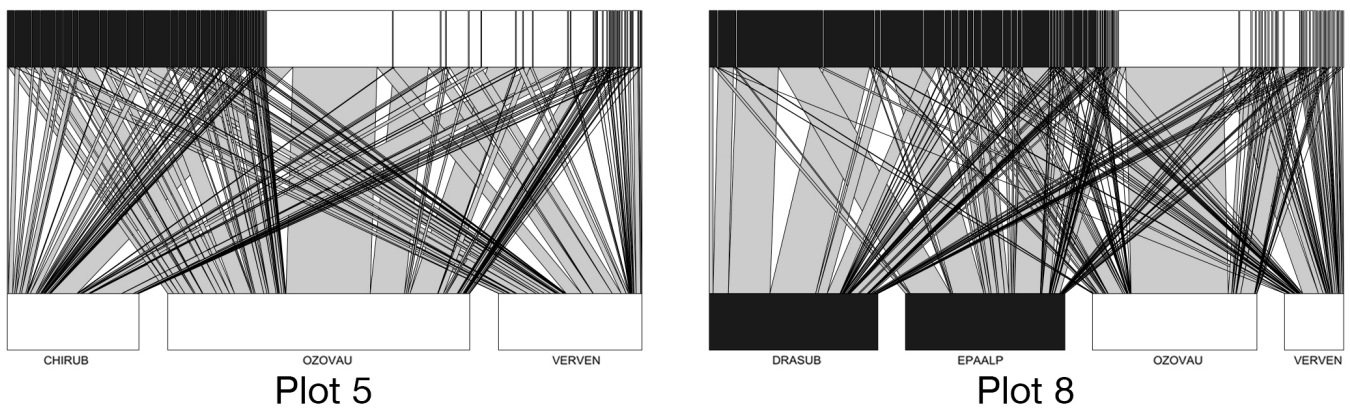

Plot 8

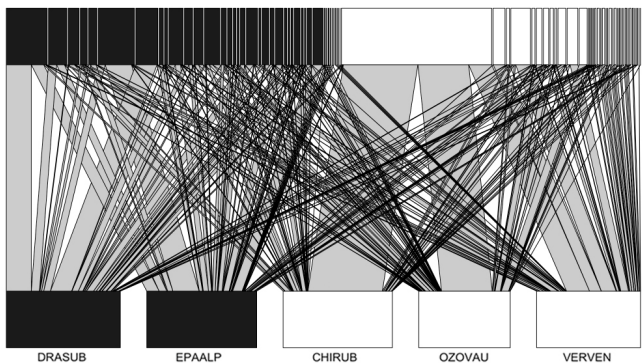

Plot 10

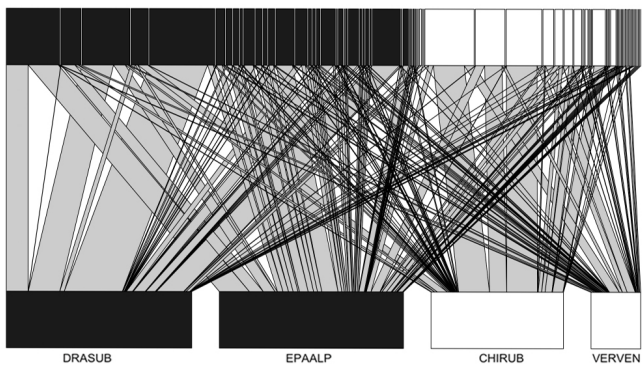

Plot 14

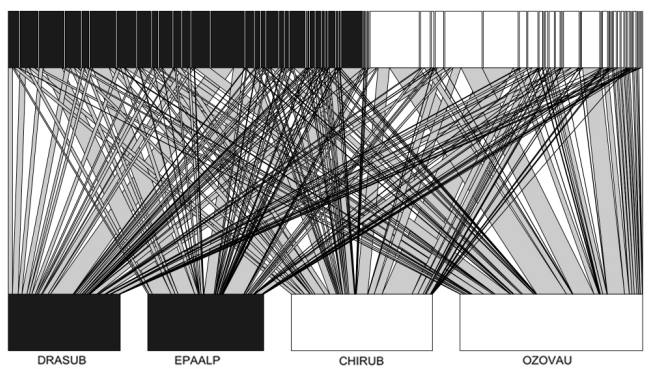

Plot 12

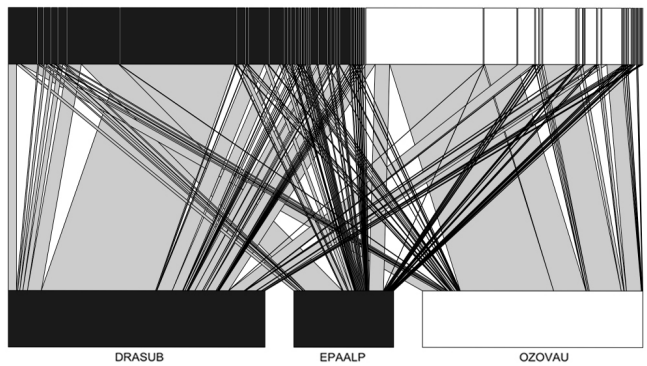

Plot 16

Supplementary Figure 4. Bipartite plant-mycorrhizal networks by plots of the "warmremoval" treatment $(\mathbf{n}=\mathbf{8})$ constructed with the plotweb function from the bipartite package in R. Host plants are on the "lower level" (lower trophic rank), connected to the ErM and AM fungal OTUs in the "higher level" (higher trophic rank). The width of the bar for each species (i.e., node) is proportional to the relative abundance of interactions it has, and acts as a proxy for the relative influence a species exerts on the network. The width of each link is proportional to the relative strength of the interaction, quantified as the number of fungal OTU sequence reads linking the fungal OTU and plant species nodes. Fungal OTUs are ordered from left to right firstly by ErM and AM fungi, and then in order of their overall rank abundance across all samples. CALVUL, Calluna vulgaris. DRASUB, Dracophyllum subulatum. EPAALP, Epacris alpina. CHIRUB, Chionochloa rubra. OZOVAU, Ozothamnus vauvilliersii. VERVEN, Veronica venustula. 


\section{BIBLIOGRAPHY}

Albrecht, M., Padrón, B., Bartomeus, I. \& Traveset, A. Consequences of plant invasions on compartmentalization and species' roles in plant-pollinator networks. Proceedings of the Royal Society B: Biological Sciences 281, 20140773 (2014).

Alexander, J. M. et al., Lags in the response of mountain plant communities to climate change. Global Change Biology 24, 563-579 (2018).

Allen, W. J. et al., Community-level direct and indirect impacts of an invasive plant favour exotic over native species. Journal of Ecology 108, 2499-2510 (2020).

Allen, E. B., Egerton-Warburton, L. M., Hilbig, B. E. \& Valliere, J. M. Interactions of arbuscular mycorrhizal fungi, critical loads of nitrogen deposition, and shifts from native to invasive species in a southern California shrubland. Botany 94, 425-433 (2016).

Almeida-Neto, M., Guimarães, P., Guimarães, P. R., Loyola, R. D. \& Ulrich, W. A consistent metric for nestedness analysis in ecological systems: reconciling concept and measurement. Oikos 117, 1227-1239 (2008)

Alpert, P., Bone, E. \& Holzapfel, C. Invasiveness, invasibility and the role of environmental stress in the spread of non-native plants. Perspectives in Plant Ecology, Evolution and Systematics 3, 52-66 (2000).

Anderson, R. C. et al., Effect of Removal of Garlic Mustard (Alliaria petiolata, Brassicaeae) on Arbuscular Mycorrhizal Fungi Inoculum Potential in Forest Soils. The Open Ecology Journal 3, 41-47 (2010).

Aprahamian, A. M. et al., Arbuscular mycorrhizal inoculation in coastal sage scrub restoration1. Botany 94, 493-499 (2016).

Augé, R. M. Water relations, drought and vesicular-arbuscular mycorrhizal symbiosis. Mycorrhiza 11, 3-42 (2001).

Averill, C., Bhatnagar, J. M., Dietze, M. C., Pearse, W. D. \& Kivlin, S. N. Global imprint of mycorrhizal fungi on whole-plant nutrient economics. Proceedings of the National Academy of Sciences 116, 23163-23168 (2019). 
Awaydul, A. et al., Common mycorrhizal networks influence the distribution of mineral nutrients between an invasive plant, Solidago canadensis, and a native plant, Kummerowa striata. Mycorrhiza 29, 29-38 (2019).

Babikova, Z. et al., Underground signals carried through common mycelial networks warn neighbouring plants of aphid attack. Ecology Letters 16, 835-843 (2013).

Badano, E. I., Villarroel, E., Bustamante, R. O., Marquet, P. A. \& Cavieres, L. A. Ecosystem engineering facilitates invasions by exotic plants in high-Andean ecosystems. Journal of Ecology 95, 682-688 (2007).

Barto, K., Friese, C. \& Cipollini, D. Arbuscular Mycorrhizal Fungi Protect a Native Plant from Allelopathic Effects of an Invader. Journal of Chemical Ecology 36, 351-360 (2010).

Barto, E. K., Weidenhamer, J. D., Cipollini, D. \& Rillig, M. C. Fungal superhighways: do common mycorrhizal networks enhance below ground communication? Trends in plant science 17, 633-637 (2012).

Bascompte, J., Jordano, P. \& Olesen, J. M. Asymmetric coevolutionary networks facilitate biodiversity maintenance. Science 312, 431-433 (2006).

Bazzicalupo, A. L., Bálint, M. \& Schmitt, I. Comparison of ITS1 and ITS2 rDNA in 454 sequencing of hyperdiverse fungal communities. Fungal Ecology 6, 102-109 (2013).

Beckage, B. et al., A rapid upward shift of a forest ecotone during 40 years of warming in the Green Mountains of Vermont. Proceedings of the National Academy of Sciences 105, 4197-4202 (2008).

Beckett, S. J. Improved community detection in weighted bipartite networks. Royal Society Open Science 3, 140536 (2016).

Bellgard, S. \& Williams, S. Response of Mycorrhizal Diversity to Current Climatic Changes. Diversity 3, 8-90 (2011).

Beniston, M., Diaz, H. F. \& Bradley, R. S. Climatic change at high elevation sites: An overview. Clim. Change 36, 233-251 (1997).

Bersier, L.-F., Banašek-Richter, C. \& Cattin, M.-F. Quantitative descriptors of food-web matrices. Ecology 83, 2394-2407 (2002). 
Bethlenfalvay, G. J. \& Dakessian, S. Grazing effects on mycorrhizal colonization and floristic composition of the vegetation on a semiarid range in northern Nevada. Rangeland Ecology \& Management/Journal of Range Management Archives 37, 312-316 (1984).

Bever, J. D. Feeback between Plants and Their Soil Communities in an Old Field Community. Ecology 75, 1965-1977 (1994).

Biggs, C. R. et al., Does functional redundancy affect ecological stability and resilience? A review and meta-analysis. Ecosphere 11, (2020).

Blaalid, R. et al., ITS1 versus ITS2 as DNA metabarcodes for fungi. Molecular Ecology Resources 13, 218-224 (2013).

Blüthgen, N. \& Klein, A.-M. Functional complementarity and specialisation: The role of biodiversity in plant-pollinator interactions. Basic and Applied Ecology 12, 282-291 (2011).

Blüthgen, N. \& Menzel, F. Measuring specialization in species interaction networks. BMC Ecology 6, 9 (2006).

Blüthgen, N. Why network analysis is often disconnected from community ecology: A critique and an ecologist's guide. Basic and Applied Ecology 11, 185-195 (2010).

Bobbink, R. et al., Global assessment of nitrogen deposition effects on terrestrial plant diversity: a synthesis. Ecological Applications 20, 30-59 (2010).

Bogar, L. M. \& Kennedy, P. G. New wrinkles in an old paradigm: neighborhood effects can modify the structure and specificity ofAlnus-associated ectomycorrhizal fungal communities. FEMS Microbiology Ecology 83, 767-777 (2013).

Bonan, G. B. Forests and Climate Change: Forcings, Feedbacks, and the Climate Benefits of Forests. Science 320, 1444-1449 (2008).

Bowman, W. D., Theodose, T. A., Schardt, J. C. \& Conant, R. T. Constraints of Nutrient Availability on Primary Production in Two Alpine Tundra Communities. Ecology 74, 2085-2097 (1993).

Bret-Harte, M. S. et al., Plant and soil responses to neighbour removal and fertilization in Alaskan tussock tundra. Journal of Ecology 92, 635-647 (2004). 
Bronstein, J. L. The evolution of facilitation and mutualism. Journal of Ecology 97, 1160-1170 (2009).

Brooker, R. W. Plant-plant interactions and environmental change. New Phytologist 171, 271-284 (2006).

Brook, B. W., Sodhi, N. S. \& Bradshaw, C. J. A. Synergies among extinction drivers under global change. Trends in Ecology \& Evolution 23, 453-460 (2008).

Brualdi, R. A. \& Sanderson, J. G. Nested species subsets, gaps, and discrepancy. Oecologia 119, 256-264 (1999).

Brundrett, M. C. Mycorrhizal associations and other means of nutrition of vascular plants: understanding the global diversity of host plants by resolving conflicting information and developing reliable means of diagnosis. Plant and Soil 320, 37-77 (2009).

Bücking, H., Mensah, J. A. \& Fellbaum, C. R. Common mycorrhizal networks and their effect on the bargaining power of the fungal partner in the arbuscular mycorrhizal symbiosis. Communicative \& Integrative Biology 9, e1107684 (2016).

Bunn, R. A., Lekberg, Y., Gallagher, C., Rosendahl, S. \& Ramsey, P. W. Grassland invaders and their mycorrhizal symbionts: a study across climate and invasion gradients. Ecology and Evolution 4, 794-805 (2014).

Bunn, R. A., Ramsey, P. W. \& Lekberg, Y. Do native and invasive plants differ in their interactions with arbuscular mycorrhizal fungi? A meta-analysis. Journal of Ecology 103, 1547-1556 (2015).

Burkle, L. A. \& Alarcon, R. The future of plant-pollinator diversity: Understanding interaction networks across time, space, and global change. American Journal of Botany 98, 528-538 (2011).

Busby, R. R., Stromberger, M. E., Rodriguez, G., Gebhart, D. L. \& Paschke, M. W. Arbuscular mycorrhizal fungal community differs between a coexisting native shrub and introduced annual grass. Mycorrhiza 23, 129-141 (2013).

Cadotte, M. W., Carscadden, K. \& Mirotchnick, N. Beyond species: functional diversity and the maintenance of ecological processes and services. Journal of Applied Ecology $\mathbf{4 8 ,}$ 1079-1087 (2011). 
Callaway, R. M. et al., Positive interactions among alpine plants increase with stress. Nature 417, 844-848 (2002).

Cameron, D. D., Neal, A. L., Van Wees, S. C. M. \& Ton, J. Mycorrhiza-induced resistance: more than the sum of its parts? Trends in Plant Science 18, 539-545 (2013).

Cannone, N., Sgorbati, S. \& Guglielmin, M. Unexpected impacts of climate change on alpine vegetation. Frontiers in Ecology and the Environment 5, 360-364 (2007).

Chagnon, P., Bradley, R. L. \& Klironomos, J. N. Mycorrhizal network assembly in a community context: The presence of neighbours matters. Journal of Ecology 108, 366377 (2020).

Chagnon, P. L., Bradley, R. L. \& Klironomos, J. N. Plant-fungal symbioses as ecological networks: the need to characterize more than just interaction patterns. Fungal Ecology 12, 10-13 (2014).

Chagnon, P.-L. \& Bainard, L. D. Is root DNA a reliable proxy to assess arbuscular mycorrhizal community structure? Can. J. Microbiol. 60, 619-624 (2014).

Chagnon, P.-L. Seeing networks for what they are in mycorrhizal ecology. Fungal Ecology 24, 148-154 (2016).

Chapman, H. \& Bannister, P. The spread of heather, Calluna vulgaris (L.) Hull, into indigenous plant communities of Tongariro National Park. N.Z. J. Ecol. 14, (1990).

Chappell, P.R. The climate and weather of Manawatu-Wanganui. NIWA Science and Technology Series 66, 40 (2015)

Cheng, D.-L. \& Niklas, K. J. Above- and Below-ground Biomass Relationships Across 1534 Forested Communities. 99, 95-102 (2007).

Cheng, G. et al., Increased precipitation, rather than warming, exerts a strong influence on arbuscular mycorrhizal fungal community in a semiarid steppe ecosystem1. Botany 94, 459-469 (2016).

Choler, P., Michalet, R. \& Callaway, R. M. Facilitation and competition on gradients in alpine plant communities. Ecology 82, 3295-3308 (2001).

Clavel, J. et al., The role of arbuscular mycorrhizal fungi in nonnative plant invasion along mountain roads. New Phytologist Early View (2020) doi:10.1111/nph.16954. 
Cole, J. R. et al., Ribosomal Database Project: data and tools for high throughput rRNA analysis. Nucleic Acids Research 42, D633-D642 (2014).

de Boer, R. et al., Improved detection of microbial DNA after bead-beating before DNA isolation. Journal of Microbiological Methods, 80, 209-211 (2010).

Deslippe, J. R., Hartmann, M., Simard, S. W. \& Mohn, W. W. Long-term warming alters the composition of Arctic soil microbial communities. FEMS Microbiology Ecology 82, 303-315 (2012).

Devoto, M., Bailey, S., Craze, P. \& Memmott, J. Understanding and planning ecological restoration of plant-pollinator networks. Ecology Letters 15, 319-328 (2012).

Díaz, S., Symstad, A. J., Stuart Chapin, F., III, Wardle, D. A. \& Huenneke, L. F. Functional diversity revealed by removal experiments. Trends in Ecology \& Evolution 18, 140-146 (2003).

Dickie, I. A., Bolstridge, N., Cooper, J. A. \& Peltzer, D. A. Co-invasion by Pinus and its mycorrhizal fungi. New Phytologist 187, 475-484 (2010).

Dormann, C. F., Fründ, J. \& Schaefer, H. M. Identifying Causes of Patterns in Ecological Networks: Opportunities and Limitations. Annual Review of Ecology, Evolution, and Systematics 48, 559-584 (2017).

Dormann, C. F., Frund, J., Bluthgen, N. \& Gruber, B. Indices, Graphs and Null Models: Analyzing Bipartite Ecological Networks. The Open Ecology Journal 2, 7-24 (2009).

Dormann, C. F. \& Strauss, R. A method for detecting modules in quantitative bipartite networks. Methods in Ecology and Evolution 5, 90-98 (2014).

Duell, E. B., Wilson, G. W. T. \& Hickman, K. R. Above- and below-ground responses of native and invasive prairie grasses to future climate scenarios1. Botany 94, 471-479 (2016).

Fatichi, S., Rimkus, S., Burlando, P., Bordoy, R. \& Molnar, P. High-resolution distributed analysis of climate and anthropogenic changes on the hydrology of an Alpine catchment. Journal of Hydrology 525, 362-382 (2015).

Ferlian, O. et al., Growing Research Networks on Mycorrhizae for Mutual Benefits. Trends in Plant Science 23, 975-984 (2018). 
Flanagan, L. B., Sharp, E. J. \& Letts, M. G. Response of plant biomass and soil respiration to experimental warming and precipitation manipulation in a Northern Great Plains grassland. Agricultural and Forest Meteorology 173, 40-52 (2013).

Geml, J. et al., Long-term warming alters richness and composition of taxonomic and functional groups of arctic fungi. FEMS Microbiology Ecology 91, 95-110 (2015).

Giejsztowt, J., Classen, A. T. \& Deslippe, J. R. Climate change and invasion may synergistically affect native plant reproduction. Ecology 101, (2020).

Gleixner, G., Danier, H. J., Werner, R. A. \& Schmidt, H. L. Correlations between the 13C Content of Primary and Secondary Plant Products in Different Cell Compartments and That in Decomposing Basidiomycetes. Plant Physiology 102, 1287-1290 (1993).

Grabherr, G., Gottfried, M. \& Pauli, H. Climate Change Impacts in Alpine Environments. Geography Compass 4, 1133-1153 (2010).

Grilli, J., Rogers, T. \& Allesina, S. Modularity and stability in ecological communities. Nature communications 7, 1-10 (2016).

Grime, J. P. Evidence for the Existence of Three Primary Strategies in Plants and Its Relevance to Ecological and Evolutionary Theory. The American Naturalist 111, 11691194 (1977).

Grove, S., Haubensak, K. A., Gehring, C. \& Parker, I. M. Mycorrhizae, invasions, and the temporal dynamics of mutualism disruption. Journal of Ecology 105, 1496-1508 (2017).

Guether, M. et al., A Mycorrhizal-Specific Ammonium Transporter from Lotus japonicus Acquires Nitrogen Released by Arbuscular Mycorrhizal Fungi. Plant Physiology 150, 73-83 (2009).

Guido, A. \& Pillar, V. D. Invasive plant removal: assessing community impact and recovery from invasion. Journal of Applied Ecology 54, 1230-1237 (2017).

Gweon, H. S. et al., PIPITS: an automated pipeline for analyses of fungal internal transcribed spacer sequences from the Illumina sequencing platform. Methods in Ecology and Evolution 6, 973-980 (2015). 
Halloy, S. R. \& Mark, A. F. Climate-change effects on alpine plant biodiversity: a New Zealand perspective on quantifying the threat. Arctic, Antarctic, and Alpine Research 35, 248-254 (2003).

Hart, M. M., Reader, R. J. \& Klironomos, J. N. Life-history strategies of arbuscular mycorrhizal fungi in relation to their successional dynamics. Mycologia 93, 1186-1194 (2001).

Hausmann, N. T. \& Hawkes, C. V. Plant neighborhood control of arbuscular mycorrhizal community composition. New Phytologist 183, 1188-1200 (2009).

Hawkes, C. V., Hartley, I. P., Ineson, P. \& Fitter, A. H. Soil temperature affects carbon allocation within arbuscular mycorrhizal networks and carbon transport from plant to fungus. Global Change Biology 14, 1181-1190 (2008).

Hegland, S. J. Floral neighbourhood effects on pollination success in red clover are scale-dependent. Functional ecology 28, 561-568 (2014).

Heinemeyer, A. et al., Impact of soil warming and shading on colonization and community structure of arbuscular mycorrhizal fungi in roots of a native grassland community. Global Change Biology 10, 52-64 (2004).

Hendershot, J. N., Read, Q. D., Henning, J. A., Sanders, N. J. \& Classen, A. T. Consistently inconsistent drivers of microbial diversity and abundance at macroecological scales. Ecology 98, 1757-1763 (2017).

He, X.-H., Critchley, C. \& Bledsoe, C. Nitrogen transfer within and between plants through common mycorrhizal networks (CMNs). Critical Reviews in plant sciences 22 , 531-567 (2003).

Horne, R. S. C. Seasonal and altitudinal variations in diet and abundance of the European hare (Lepus europaeus Pallas) in Tongariro National Park, New Zealand: a thesis for the degree of Master of Science in Zoology at Massey University. vol. Master of Science (M. Sc.) (Massey University, 1979).

Hoyle, G. L. et al., Soil warming increases plant species richness but decreases germination from the alpine soil seed bank. Global Change Biology 19, 1549-1561 (2013). 
Hülber, K. et al., Uncertainty in predicting range dynamics of endemic alpine plants under climate warming Global Change Biology. 22, 2608-2619 (2016).

IPCC, 2014: Climate Change 2014: Synthesis Report. Contribution of Working Groups I, II and III to the Fifth Assessment Report of the Intergovernmental Panel on Climate Change [Core Writing Team, R.K. Pachauri and L.A. Meyer (eds.)]. IPCC, Geneva, Switzerland, 151.

James, A., Pitchford, J. W. \& Plank, M. J. Disentangling nestedness from models of ecological complexity. Nature 487, 227-230 (2012).

Jasper, D. A., Abbott, L. K. \& Robson, A. D. Soil disturbance reduces the infectivity of external hyphae of vesicular-arbuscular mycorrhizal fungi. New Phytologist 112, 93-99 (1989).

Jing, X. et al., The links between ecosystem multifunctionality and above and belowground biodiversity are mediated by climate. Nature Communications $\mathbf{6}, 8159$ (2015).

Johnson, N. C. \& Graham, J. H. The continuum concept remains a useful framework for studying mycorrhizal functioning. Plant and Soil 363, 411-419 (2013).

Johnson, N. C., Graham, J. H. \& Smith, F. A. Functioning of mycorrhizal associations along the mutualism-parasitism continuum. New Phytologist 135, 575-585 (1997).

Johnson, D. \& Gilbert, L. Interplant signalling through hyphal networks. New Phytologist 205, 1448-1453 (2015).

Keesing, V. F. Impacts of invasion on community structure: habitat and invertebrate assemblage responses to Calluna vulgaris (L.) Hull invasion, in Tongariro National Park, New Zealand: a thesis presented in partial fulfilment of the requirements for the degree of Doctor of Philosophy in Zoology at Massey University, Palmerston North. vol. Doctor of Philosophy (Ph.D.) (Massey University, 1995).

Kikvidze, Z. et al., Linking patterns and processes in alpine plant communities: A global study. Ecology 86, 1395-1400 (2005).

Kilpeläinen, J., Aphalo, P. J. \& Lehto, T. Temperature affected the formation of arbuscular mycorrhizas and ectomycorrhizas in Populus angustifolia seedlings more than a mild drought. Soil Biology and Biochemistry 146, 77- 98 (2020). 
Klanderud, K. Climate change effects on species interactions in an alpine plant community. Journal of Ecology 93, 127-137 (2005).

Klanderud, K. Species-specific responses of an alpine plant community under simulated environmental change. Journal of Vegetation Science 19, 363-372 (2008).

Klink, S., Giesemann, P., Hubmann, T. \& Pausch, J. Stable C and N isotope natural abundances of intraradical hyphae of arbuscular mycorrhizal fungi. Mycorrhiza 30, 773780 (2020).

Korbie, D. J. \& Mattick, J. S. Touchdown PCR for increased specificity and sensitivity in PCR amplification. Nature Protocols 3, 1452-1456 (2008).

Kostenko, O., Van De Voorde, T. F. J., Mulder, P. P. J., Van Der Putten, W. H. \& Martijn Bezemer, T. Legacy effects of aboveground-belowground interactions. Ecology Letters 15, 813-821 (2012).

Kou-Giesbrecht, S. \& Menge, D. Nitrogen-fixing trees could exacerbate climate change under elevated nitrogen deposition. Nature Communications 10, (2019).

Kullman, L. A Richer, Greener and Smaller Alpine World: Review and Projection of Warming-Induced Plant Cover Change in the Swedish Scandes. AMBIO 39, 159-169 (2010).

Lai, S.-M., Liu, W.-C. \& Jordán, F. On the centrality and uniqueness of species from the network perspective. Biology Letters 8, 570-573 (2012).

Lankau, R. Soil microbial communities alter allelopathic competition between Alliaria petiolata and a native species. Biological Invasions 12, 2059-2068 (2010).

Legay, N. et al., Soil legacy effects of climatic stress, management and plant functional composition on microbial communities influence the response of Lolium perenne to a new drought event. Plant and Soil 424, 233-254 (2018).

Lehmann, J. et al., Biochar effects on soil biota - A review. Soil Biology and Biochemistry 43, 1812-1836 (2011).

Lekberg, Y., Gibbons, S. M., Rosendahl, S. \& Ramsey, P. W. Severe plant invasions can increase mycorrhizal fungal abundance and diversity. The ISME Journal 7, 1424-1433 (2013). 
Le Roux, J. J. et al., Importance of soil legacy effects and successful mutualistic interactions during Australian acacia invasions in nutrient-poor environments. Journal of Ecology 106, 2071-2081 (2018) doi:10.1111/1365-2745.12965.

Loarie, S. R. et al., The velocity of climate change. Nature 462, 1052-1055 (2009).

Ma, X. et al., Global negative effects of nutrient enrichment on arbuscular mycorrhizal fungi, plant diversity and ecosystem multifunctionality. Early View New Phytologist (2020) doi:10.1111/nph.17077.

Mantyka-Pringle, C. S., Martin, T. G. \& Rhodes, J. R. Interactions between climate and habitat loss effects on biodiversity: a systematic review and meta-analysis. Global Change Biology 18, 1239-1252 (2012).

Marín, C., Kohout, P. Response of soil fungal ecological guilds to global changes. Early View New Phytologist (2020) doi:10.1111/nph.17054.

Marion, G. M. et al., Open-top designs for manipulating field temperature in high-latitude ecosystems. Global Change Biology 3, 20-32 (1997).

Marshall, C. B., McLaren, J. R. \& Turkington, R. Soil microbial communities resistant to changes in plant functional group composition. Soil Biology and Biochemistry 43, 78-85 (2011).

Martin, M. Cutadapt removes adapter sequences from high-throughput sequencing reads. EMBnet.journal 17, 10 (2011).

Mayor, J. R. et al., Elevation alters ecosystem properties across temperate treelines globally. Nature 542, 91-95 (2017).

Mccann, K. S. The diversity-stability debate. Nature 405, 228-233 (2000).

Mccune, B. \& Grace, J. Analysis of ecological communities. MJM Software Design, Gleneden Beach, OR. (2002).

Mcdougall, K. L. et al., Alien flora of mountains: global comparisons for the development of local preventive measures against plant invasions. Diversity and Distributions 17, 103-111 (2011).

McLaren, J. R. \& Turkington, R. Biomass compensation and plant responses to 7 years of plant functional group removals. Journal of Vegetation Science 22, 503-515 (2011). 
Montesinos-Navarro, A., Segarra-Moragues, J. G., Valiente-Banuet, A. \& Verdú, M. The network structure of plant-arbuscular mycorrhizal fungi. New Phytologist 194, 536547 (2012).

Moora, M. et al., Alien plants associate with widespread generalist arbuscular mycorrhizal fungal taxa: evidence from a continental-scale study using massively parallel 454 sequencing. Journal of Biogeography 38, 1305-1317 (2011).

Mote, P. W., Hamlet, A. F., Clark, M. P. \& Lettenmaier, D. P. Declining mountain snowpack in western north America. Bull. Amer. Meteorol. Soc. 86, 39-+ (2005).

Müller, A., Ngwene, B., Peiter, E. \& George, E. Quantity and distribution of arbuscular mycorrhizal fungal storage organs within dead roots. Mycorrhiza 27, 201-210 (2017).

Muneer, M. A., Wang, P., Zaib-un-Nisa, Lin, C. \& Ji, B. Potential role of common mycorrhizal networks in improving plant growth and soil physicochemical properties under varying nitrogen levels in a grassland ecosystem. Global Ecology and Conservation 24, e01352 (2020).

Nguyen, N. H. et al., FUNGuild: An open annotation tool for parsing fungal community datasets by ecological guild. Fungal Ecology 20, 241-248 (2016).

Nilsson, R. H. et al., Mycobiome diversity: high-throughput sequencing and identification of fungi. Nature Reviews Microbiology 17, 95-109 (2019).

Nogués-Bravo, D., Araújo, M. B., Errea, M. P. \& Martínez-Rica, J. P. Exposure of global mountain systems to climate warming during the 21st Century. Global Environmental Change 17, 420-428 (2007).

Nuñez, M. A. \& Dickie, I. A. Invasive belowground mutualists of woody plants. Biological Invasions 16, 645-661 (2014).

Olesen, J. M., Bascompte, J., Dupont, Y. L. \& Jordano, P. The modularity of pollination networks. Proceedings of the National Academy of Sciences 104, 19891-19896 (2007).

Opsahl, T. \& Panzarasa, P. Clustering in weighted networks. Social Networks 31, 155163 (2009).

Orlovich, D. A. \& Cairney, Johnw. G. Ectomycorrhizal fungi in New Zealand: Current perspectives and future directions. 42, 721-738 (2004). 
Pattinson, G. S., Hammill, K. A., Sutton, B. G. \& Mcgee, P. A. Simulated fire reduces the density of arbuscular mycorrhizal fungi at the soil surface. Mycological Research 103, 491-496 (1999).

Pauchard, A. et al., Ain't no mountain high enough: plant invasions reaching new elevations. Frontiers in Ecology and the Environment 7, 479-486 (2009).

Peay, K. G. The mutualistic niche: mycorrhizal symbiosis and community dynamics. Annual Review of Ecology, Evolution, and Systematics 47, 143-164 (2016).

Pepin, N. et al., Elevation-dependent warming in mountain regions of the world. Nat. Clim. Chang. 5, 424-430 (2015).

Perotto, S., Daghino, S. \& Martino, E. Ericoid mycorrhizal fungi and their genomes: another side to the mycorrhizal symbiosis? New Phytologist 220, 1141-1147 (2018).

Perry, G. L., Wilmshurst, J. M. \& McGlone, M. S. Ecology and long-term history of fire in New Zealand. New Zealand Journal of Ecology 157-176 (2014).

Petchey, O. L. \& Gaston, K. J. Dendrograms and measuring functional diversity. 116, 1422-1426 (2007).

Petchey, O. L. \& Gaston, K. J. Functional diversity: back to basics and looking forward. Ecology Letters 9, 741-758 (2006).

Peterson, P. G., Merrett, M. F., Fowler, S. V., Barrett, D. P. \& Paynter, Q. Comparing biocontrol and herbicide for managing an invasive non-native plant species: Efficacy, non-target effects and secondary invasion. Journal of Applied Ecology 57, 1876-1884 (2020).

Pickles, B. J. \& Simard, S. W. Chapter 18 - mycorrhizal networks and forest resilience to drought. in Mycorrhizal mediation of soil (eds. Johnson, N. C., Gehring, C. \& Jansa, J.) 319-339 (2017). doi:https://doi.org/10.1016/B978-0-12-804312-7.00018-8.

Pielou, E. C. The measurement of diversity in different types of biological collections. Journal of Theoretical Biology 13, 131-144 (1966).

Pocock, M. J. O., Evans, D. M. \& Memmott, J. The Robustness and Restoration of a Network of Ecological Networks. Science 335, 973-977 (2012). 
Pugnaire, F. I. et al., Warming enhances growth but does not affect plant interactions in an alpine cushion species. Perspectives in Plant Ecology, Evolution and Systematics 44, 125530 (2020).

Read, Q. D., Henning, J. A., Classen, A. T. \& Sanders, N. J. Aboveground resilience to species loss but belowground resistance to nitrogen addition in a montane plant community. Journal of Plant Ecology 11, 351-363 (2018).

Read, D. J. \& Perez-Moreno, J. Mycorrhizas and nutrient cycling in ecosystems - a journey towards relevance? New Phytologist 157, 475-492 (2003).

Reinhart, K. O. \& Callaway, R. M. Soil biota and invasive plants. New phytologist 170, 445-457 (2006).

Richardson, D. M., Allsopp, N., D’Antonio, C. M., Milton, S. J. \& Rejmánek, M. Plant invasions - the role of mutualisms. Biological Reviews 75, 65-93 (2007).

Rodriguez-Ramos, J. C. et al., Changes in soil fungal community composition depend on functional group and forest disturbance type. New Phytologist Early View (2020) doi: $10.1111 / \mathrm{nph} .16749$.

Rogers, G. M. \& Leathwick, J. R. North Island seral tussock grasslands. 3. The influence of heather (Calluna vulgaris) on rates of change from tussock grassland to shrubland. New Zealand Journal of Botany 34, 473-487 (1996).

Rognes T, Flouri T, Nichols B, Quince C, Mahé F. (2016) VSEARCH: a versatile open source tool for metagenomics. PeerJ 4 e2584. doi:10.7717/peerj.2584

Rooney, N., Mccann, K., Gellner, G. \& Moore, J. C. Structural asymmetry and the stability of diverse food webs. Nature 442, 265-269 (2006).

Rumpf, S. B. et al., Range dynamics of mountain plants decrease with elevation. Proceedings of the National Academy of Sciences 115, 1848-1853 (2018).

Scherrer, D. \& Körner, C. Infra-red thermometry of alpine landscapes challenges climatic warming projections. Global Change Biology 2602-2613 (2009) doi: $\underline{10.1111 /}$ j.1365-2486.2009.02122.x.

Schleuning, M. et al., Ecological, historical and evolutionary determinants of modularity in weighted seed-dispersal networks. Ecology letters 17, 454-463 (2014). 
Selosse, M.-A., Richard, F., He, X. \& Simard, S. W. Mycorrhizal networks: des liaisons dangereuses? Trends in Ecology \& Evolution 21, 621-628 (2006).

Shannon, C. E. A mathematical theory of communication. The Bell system technical journal 27, 379-423 (1948).

Sheykhali, S. et al., Robustness to extinction and plasticity derived from mutualistic bipartite ecological networks. Scientific Reports 10, (2020).

Smale, M. C. Ecology of Dracophyllum subulatum - dominant heathland on frost flats at Rangitaiki and north Pureora, central North Island, New Zealand. 28, 225-248 (1990).

Smith, B. \& Wilson, J. B. A Consumer's Guide to Evenness Indices. Oikos 76, 70-82 (1996).

Smith, F. A. \& Smith, S. E. How useful is the mutualism-parasitism continuum of arbuscular mycorrhizal functioning? Plant and Soil 363, 7-18 (2013).

Smith, S. E. \& Read, D. J. Mycorrhizal symbiosis. (Academic press, 2010).

Smith, E. P. Randomization methods and the analysis of multivariate ecological data. Environmetrics 9, 37-51 (1998).

Soudzilovskaia, N. A., Vaessen, S., Van'T Zelfde, M. \& Raes, N. Global Patterns of Mycorrhizal Distribution and Their Environmental Drivers. in Biogeography of Mycorrhizal Symbiosis, 18, 223-235 (Springer International Publishing, 2017). doi:10.1007/978-3-319-56363-3 11.

Steidinger, B. S. et al., Climatic controls of decomposition drive the global biogeography of forest-tree symbioses. Nature 569, 404-408 (2019).

Stinson, K. A., Campbell, S. A., Powell, J. R., Wolfe, B. E. \& Callaway, R. M. Invasive plant suppresses the growth of native tree seedlings by disrupting belowground. PLoS Biology 4, 727-731 (2006).

Stoffel, M. \& Huggel, C. Effects of climate change on mass movements in mountain environments. Progress in Physical Geography: Earth and Environment 36, 421-439 (2012).

Stone, L. \& Roberts, A. The checkerboard score and species distributions. Oecologia 85, 74-79 (1990). 
Stone, L. \& Roberts, A. Competitive exclusion, or species aggregation? Oecologia 91, 419-424 (1992).

Sun, X. et al., Diversity of arbuscular mycorrhizal fungal spore communities and its relations to plants under increased temperature and precipitation in a natural grassland. Chinese Science Bulletin 58, 4109-4119 (2013).

Tam, P. C. F. Heavy metal tolerance by ectomycorrhizal fungi and metal amelioration by Pisolithus tinctorius. Mycorrhiza 5, 181-187 (1995).

Thakuria, D., Schmidt, O., Mac Siurtain, M., Egan, D. \& Doohan, F. M. Importance of DNA quality in comparative soil microbial community structure analyses. Soil Biology \& Biochemistry. 40, 1390-1403 (2008).

Toju, H., Guimarães, P. R., Olesen, J. M. \& Thompson, J. N. Assembly of complex plantfungus networks. Nature Communications 5, 5273 (2014).

Toju, H. High-throughput DNA barcoding for ecological network studies. Population Ecology 57, 37-51 (2015).

Toju, H. \& Sato, H. Root-Associated Fungi Shared Between Arbuscular Mycorrhizal and Ectomycorrhizal Conifers in a Temperate Forest. Frontiers in Microbiology 9, (2018).

Trivedi, M. R., Berry, P. M., Morecroft, M. D. \& Dawson, T. P. Spatial scale affects bioclimate model projections of climate change impacts on mountain plants. Global Change Biology 14, 1089-1103 (2008).

Tylianakis, J. M., Tscharntke, T. \& Lewis, O. T. Habitat modification alters the structure of tropical host-parasitoid food webs. Nature 445, 202-205 (2007).

Ulrich, W., Almeida-Neto, M. \& Gotelli, N. J. A consumer's guide to nestedness analysis. Oikos 118, 3-17 (2009).

van Der Heijden, M. G. A. \& Horton, T. R. Socialism in soil? The importance of mycorrhizal fungal networks for facilitation in natural ecosystems. Journal of Ecology 97, 1139-1150 (2009).

van der Putten, W. H., Klironomos, J. N. \& Wardle, D. A. Microbial ecology of biological invasions. The ISME journal 1, 28-37 (2007). 
van't Padje, A., Werner, G. D. A. \& Kiers, E. T. Mycorrhizal fungi control phosphorus value in trade symbiosis with host roots when exposed to abrupt 'crashes' and 'booms' of resource availability. New Phytologist Early View (2020) doi: 10.1111/nph.17055.

Vogelsang, K. M. \& Bever, J. D. Mycorrhizal densities decline in association with nonnative plants and contribute to plant invasion. Ecology 90, 399-407 (2009).

Wahl, A.-L. \& Spiegelberger, T. Arbuscular mycorrhizal fungi in changing mountain grassland ecosystems: a challenge for research. Botany 94, 435-458 (2016).

Wang, Q., Garrity, G. M., Tiedje, J. M. \& Cole, J. R. Naïve Bayesian Classifier for Rapid Assignment of rRNA Sequences into the New Bacterial Taxonomy. Applied and Environmental Microbiology 73, 5261-5267 (2007).

Wirth, L. R. et al., Effects of floral neighborhood on seed set and degree of outbreeding in a high-alpine cushion plant. Oecologia 167, 427-434 (2011).

$\mathrm{Xu}, \mathrm{X}$. et al., Dominant plant species shift their nitrogen uptake patterns in response to nutrient enrichment caused by a fungal fairy in an alpine meadow. Plant and Soil 341, 495-504 (2011).

Yang, G., Wagg, C., Veresoglou, S. D., Hempel, S. \& Rillig, M. C. How Soil Biota Drive Ecosystem Stability. Trends in Plant Science 23, 1057-1067 (2018).

Zhang, J., Kobert, K., Flouri, T. \& Stamatakis, A. PEAR: a fast and accurate Illumina Paired-End reAd mergeR. Bioinformatics 30, 614-620 (2014b).

Zhang, Q. et al., Positive feedback between mycorrhizal fungi and plants influences plant invasion success and resistance to invasion. PLoS one 5, e12380 (2010).

Zhang, B. et al., Responses of Soil Microbial Communities to Experimental Warming in Alpine Grasslands on the Qinghai-Tibet Plateau. PLoS one 9, e103859 (2014a).

Zobel, M. \& Öpik, M. Plant and arbuscular mycorrhizal fungal (AMF) communities which drives which? Journal of Vegetation Science 25, 1133-1140 (2014).

Zuppinger-Dingley, D., Flynn, D. F. B., De Deyn, G. B., Petermann, J. S. \& Schmid, B. Plant selection and soil legacy enhance long-term biodiversity effects. Ecology 97, 918928 (2016). 
